\title{
DIGITALLY CONTROLLED ENERGY HARVESTING POWER MANAGEMENT SYSTEM
}

By

Andrew Jordan Dickson

A thesis submitted to the Department of Electrical \& Computer Engineering

In conformity with the requirements for

the degree of Master of Applied Science

\author{
Queen’s University \\ Kingston, Ontario, Canada
}

March, 2012

Copyright (C) Andrew Jordan Dickson, 2012 


\begin{abstract}
This thesis discusses a power electronics module (PEM) that is used to extract power from a human energy harvesting generator according to the user's desired input power, and stores all of the extracted energy into an appropriately sized battery while staying within the charging limitations of the battery. The PEM can temporarily store the peak power produced by the generator allowing the reduction in the size of the battery required to the average power production level of the generator. The battery's safety and longevity is maintained by charging them at the constant current and constant voltage rate.
\end{abstract}

The design of the two-stage PEM, the requirements of the Energy Storage Capacitor (ESC) and battery size are discussed. The two controllers that control the PEM are explained and the different operating modes of the controllers are reviewed. A two-stage prototype digitally controlled average current mode control Boost converter and average current mode controlled Buck converter were designed and experimental waveforms were captured to test and validate the control theories used in the PEM. A Voltage Adaptive Gain compensator was used to optimize the closed loop response of both the Boost and Buck converters over their respective output and input voltage ranges. The DC efficiency of the prototype was measured with the peak efficiency of the Boost converter equal to $93 \%$ and the peak efficiency of the Buck converter measured at 93.7\%. The total PEM system efficiency was measured at $87.9 \%$ at an input power level of 10 watts. The AC efficiency of the PEM was also measured with a peak efficiency of $91 \%$ with $V_{\text {in }}=15 \mathrm{~V}$ at $R_{\text {in }}=60 \Omega$.

The software considerations for an embedded system, including power consumption and timing of real time events are reviewed. A software flow chart and timing diagram are provided to help 
visualize the sequence of the code. A design chart for selection of the size and voltage rating of the ESC was created. An experimental comparison of a single stage design without energy storage capability and the current PEM design was performed, with a power limited source, in order to show the effectiveness of the PEM and controllers at maximizing the power extraction from the generator. The PEM design was able to extract 50\% more power than the single stage converter without energy storage capability. 


\section{Acknowledgements}

First of all I would like to thank not only my supervisor, but also my mentor through this process, Dr. Yan-Fei Liu. He has provided me with structure and encouragement throughout the duration of my degree. Secondly, I would like to give a special thanks to my partner in designing, prototyping, and testing of the human energy harvester. Michael Sherpertycky has been an excellent support as well as a good friend. I was also surrounded by great lab mates, who have become friends throughout the process, and offered valuable insight.

I would also like to thank my parents, Cindy and Russell Dickson, as well as my sisters Jenna, Jasmine and Ashley, and my brother in law William Shaw. They have always been there for me and I know they will continue to be. I would lastly like to thank my girlfriend, Rebecca Sloan for her unconditional encouragement and support throughout this process. 


\section{Table of Contents}

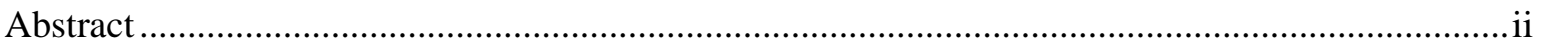

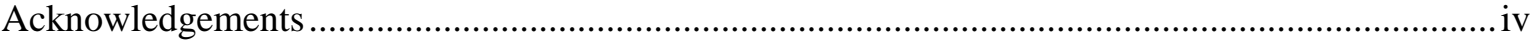

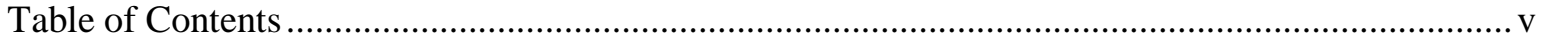

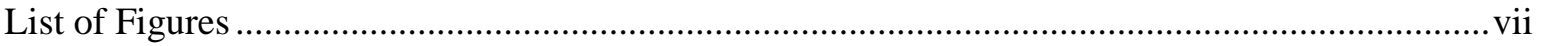

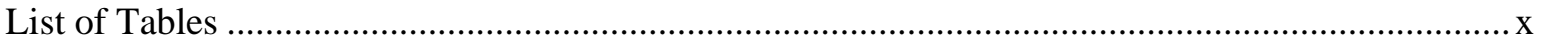

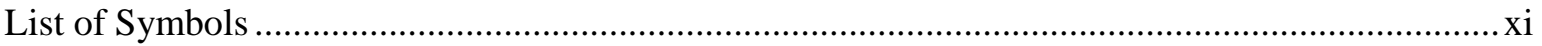

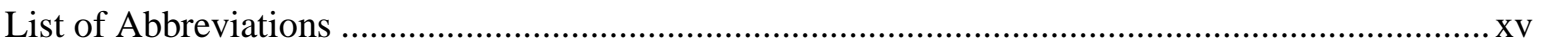

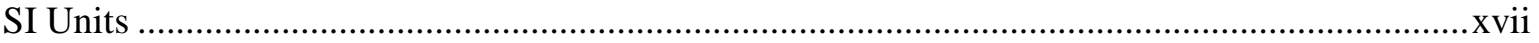

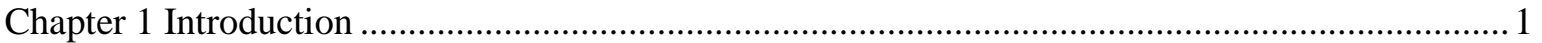

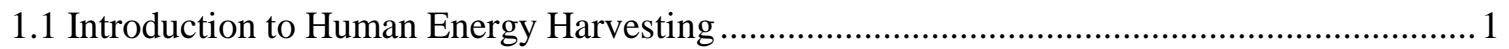

1.2 Previous Works on Human Energy Harvesting Generator Designs and Power Electronics

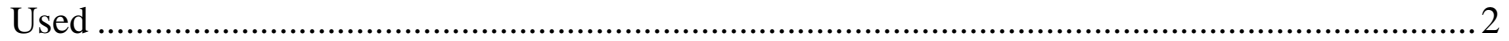

1.2.1 Human Energy Harvesting Generator Designs .............................................................. 2

1.2.2 Power Electronic Circuitry used in Human Energy Harvesting......................................... 5

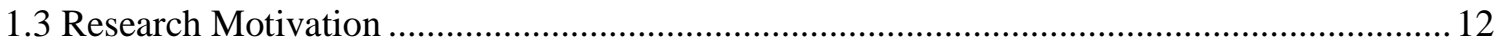

1.3.1 Maximize the Amount of Power Extracted from the Generator While Controlling the

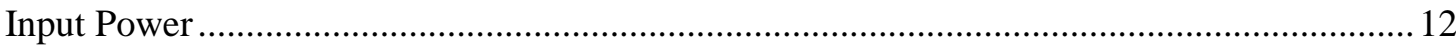

1.3.2 Store Extracted Power into Batteries While Not Exceeding the Charging Limitations of an Appropriately Sized Lithium Polymer Battery................................................................. 13

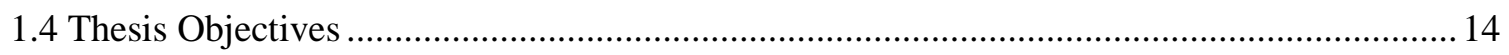

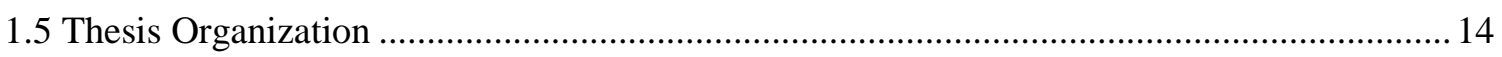

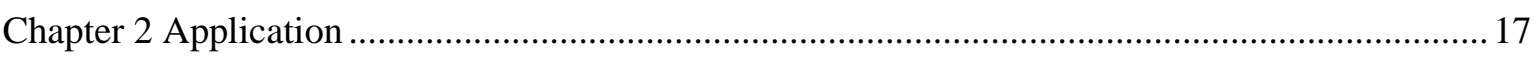

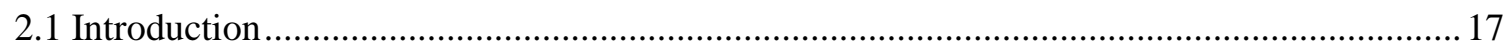

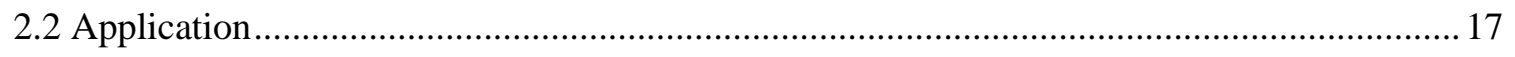

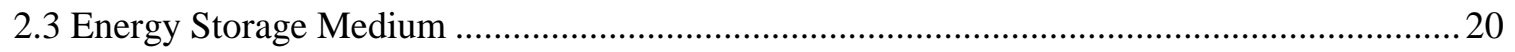

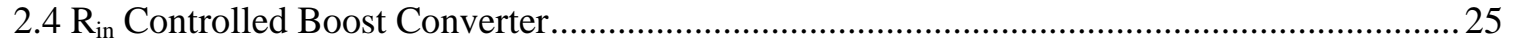

2.5 Maximum Energy Extraction and Charge Controlled Buck Converter ................................... 29

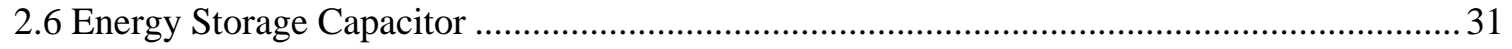

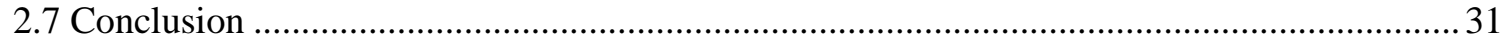


Chapter 3 Control Strategy of $\mathrm{R}_{\mathrm{in}}$ Controlled Boost Converter .....

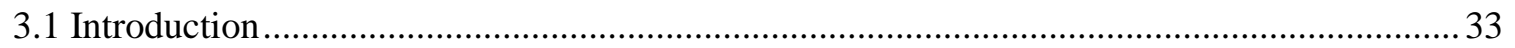

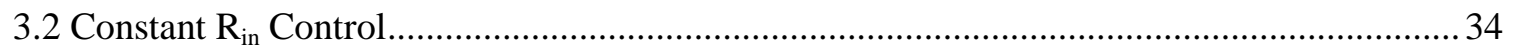

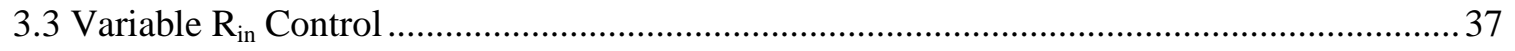

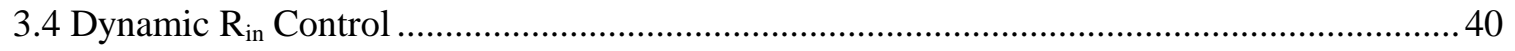

3.5 Prototype Requirements and Design of $\mathrm{R}_{\text {in }}$ Controlled Boost Converter................................ 43

3.6 Digital Average Current Mode Controlled Boost Converter Plant Transfer Function ........... 47

3.7 Boost Two-Pole Two-Zero Compensator with Voltage Adaptive Compensator Gain............ 56

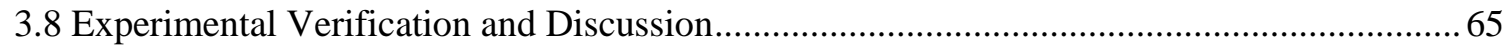

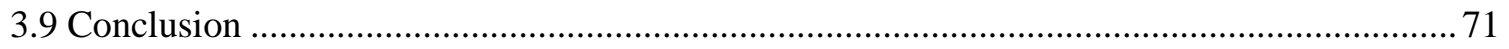

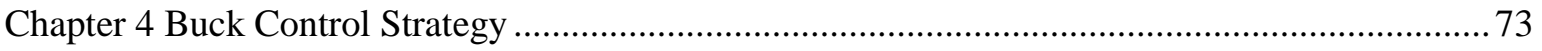

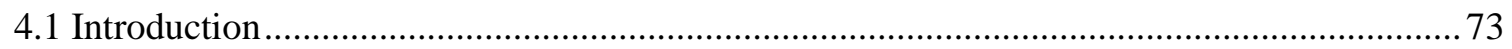

4.2 Buck Maximum Energy Extraction and Charge Control Scheme .......................................... 73

4.3 Digitally Controlled Buck Converter Plant Transfer Function .............................................. 80

4.4 Buck Two-Pole Two-Zero Compensator with Voltage Adaptive Gain ..................................85

4.5 Driving of Synchronous Buck Control FET without the use of an Isolated Supply Voltage .92

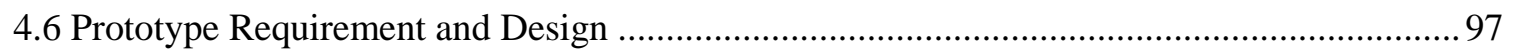

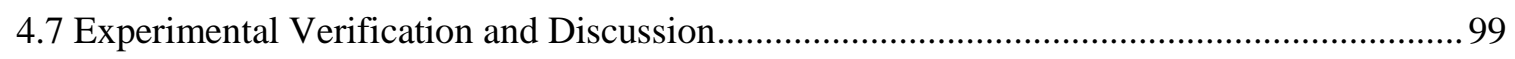

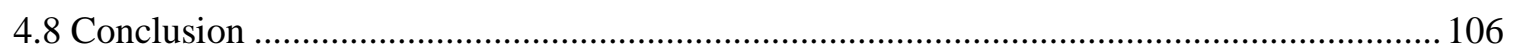

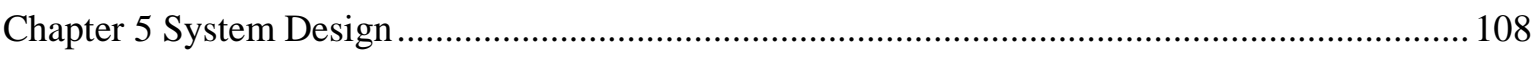

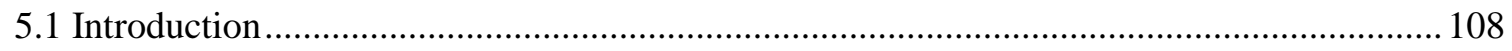

5.2 Embedded System Software Considerations and ISR Execution Timing.............................108

5.3 Selection of the Size and Voltage of the Energy Storage Capacitor versus Input Power ..... 116

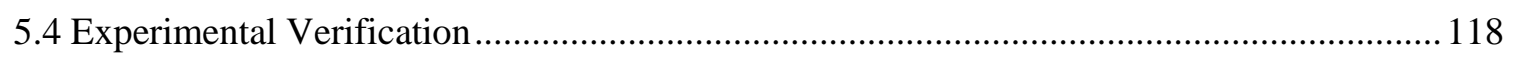

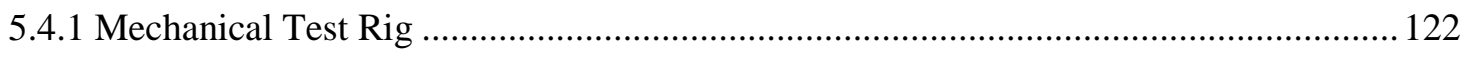

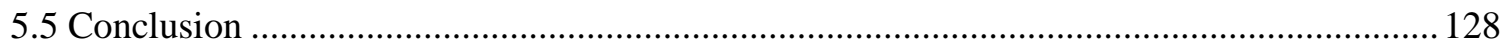

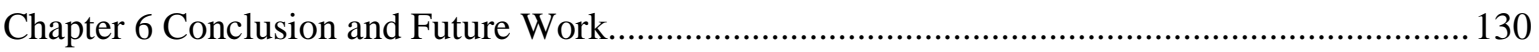

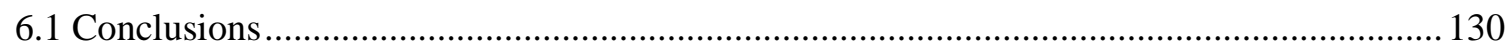

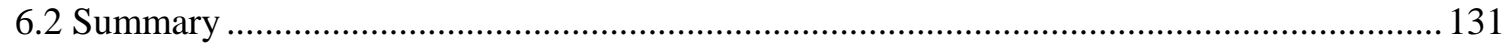

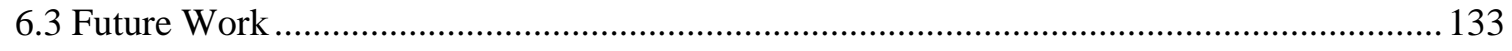

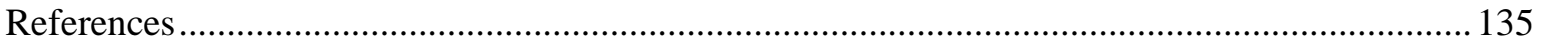




\section{List of Figures}

Figure 1-1 - Biomechanical Knee Brace Energy Harvester [11] .................................................... 3

Figure 1-2 - Backpack Based Linear Motion Energy Harvesting System [13] ................................ 5

Figure 1-3 - Mini-Vibrational Power Generator Circuit Schematic [16] ....................................... 6

Figure 1-4 - Schematic of Mechanical Oscillator Energy Harvester Backpack System [4]...............8

Figure 1-5 - Mechanical Oscillator Energy Harvester Backpack System Battery Charging Voltage

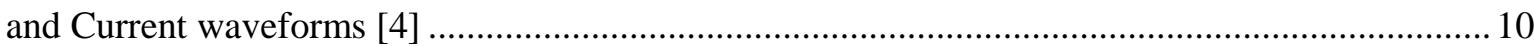

Figure 1-6 - Power Electronic Circuit used in [14] ................................................................. 11

Figure 2-1 - Typical Three-Phase AC waveform from Human Energy Harvester with user walking at $1.5 \mathrm{~m} / \mathrm{s}(5.4 \mathrm{~km} / \mathrm{h})$ 19

Figure 2-2 - Three-Phase AC waveform from Human Energy Harvester with user walking at 1.5 $\mathrm{m} / \mathrm{s}$ (Zoomed In)

Figure 2-3 - Energy Densities of Secondary Battery Chemistries [20] ..........................................2 21

Figure 2-4 - Charging profile of Lithium Batteries [21] .23

Figure 2-5 - Plot of Time Varying Input Power Generated by the Human Energy Harvester versus

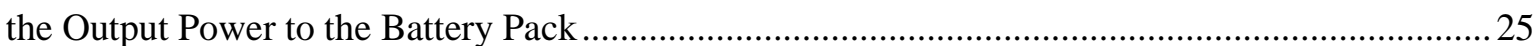

Figure 2-6 - Two-Stage Power Electronics Module with Energy Storage Capacitor.......................26

Figure 2-7 - Rectified Voltage from Generator Low Frequency AC envelope ................................22

Figure 2-8 - Rectified Voltage from Generator High frequency Super Imposed Waveform (Zoomed

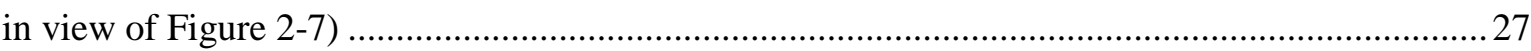

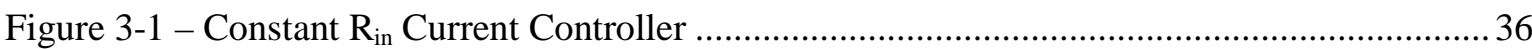

Figure 3-2 - Schematic of $\mathrm{R}_{\text {in }}$ Controlled Boost Converter .......................................................... 37

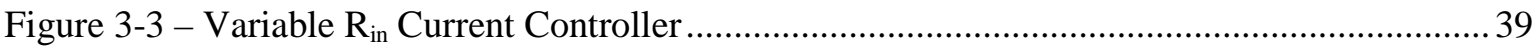

Figure 3-4 - Plot of Input Power with Variable $\mathrm{R}_{\text {in }}$ Controlled Boost Converter ............................ 40

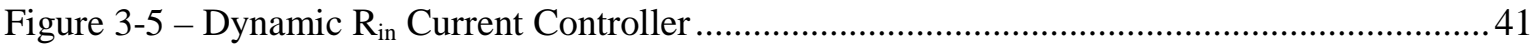

Figure 3-6 - Plot of Input Power with Dynamic $\mathrm{R}_{\text {in }}$ Controlled Boost Converter ............................. 42

Figure 3-7 - Printed Circuit Board Prototype of PEM..................................................................... 45

Figure 3-8 - Schematic of $\mathrm{R}_{\text {in }}$ Controlled Boost Converter with ADC Connections........................ 47

Figure 3-9 - Schematic of Boost Converter Operating in CCM .................................................... 48

Figure 3-10 - Boost Converter in Operating State 1....................................................................... 49

Figure 3-11 - Boost Converter in Operating State 2.................................................................... 50 
Figure 3-12 - Simplification of Linearized Block Diagram of Boost Converter operating in CCM 52

Figure 3-13 - Closed Loop Block Diagram of Digital Control System [28].

Figure 3-14 - Open Loop Bode Plot of Boost Current Loop at Different Output Voltages with a

Conventional Two-pole Two-zero Compensator. 57

Figure 3-15 - Rise Time of Inductor Current for $\mathrm{R}_{\mathrm{in}}$ Controlled Boost Converter with Conventional

Controller .59

Figure 3-16 - Slew Rate of $\mathrm{V}_{\text {Boost }}$

Figure 3-17 - Open Loop Bode Plot of Boost Current Loop at Different Output Voltages with a

Voltage Adaptive Variable Gain Compensator.

Figure 3-18 - Rise Time of Inductor Current for $\mathrm{R}_{\text {in }}$ Controlled Boost Converter with Variable Gain

Controller. . .64

Figure 3-19 - AC Input Source Schematic 65

Figure 3-20 - Input Voltage Waveform Used to test Prototype .66

Figure 3-21 - $\mathrm{R}_{\text {in }}$ Controlled Boost Converter operating in Constant $\mathrm{R}_{\text {in }}$ Control Mode. 67

Figure 3-22 - Variable $\mathrm{R}_{\text {in }}$ Control Mode 68

Figure 3-23 - Dynamic $\mathrm{R}_{\text {in }}$ Control Mode ... 69

Figure 3-24 - Boost Converter 20 Volt DC Efficiency …........................................................... 71

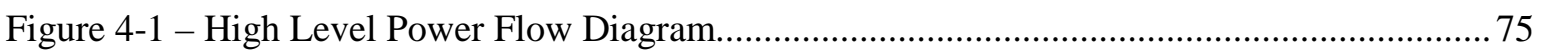

Figure 4-2 - Maximum Energy Extraction Charge Controller Block Diagram............................... 75

Figure 4-3 - Two Loop Controller for Control of Charging Current and Charging Voltage ............ 76

Figure 4-4 - Boost Voltage Controller............................................................................................ 78

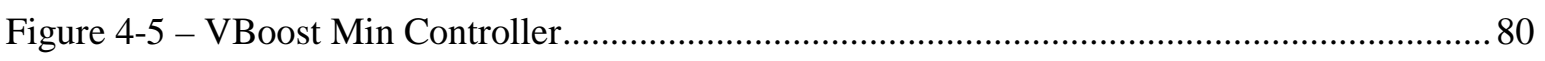

Figure 4-6 - Schematic of Buck Converter Operating in CCM................................................... 81

Figure 4-7 - Simplification of Linearized Block Diagram of Buck Converter Operating in CCM.. 83

Figure 4-8 - Continuous-Time Domain Closed Loop Buck Voltage and Current Loop ..... .84

Figure 4-9 - Open Loop Bode Plot of Buck Converter at Different Input Voltages with a

Conventional Two-pole Two-zero Compensator.

Figure 4-10 - Rise Time of Inductor Current for MEECC Controlled Buck Converter with

Conventional Controller. 88

Figure 4-11 - Open Loop Bode Plot of Buck Converter at Different Input Voltages with a Voltage

Adaptive Gain Compensator 89 
Figure 4-12 - Rise Time of Inductor Current MEECC Controlled Buck Converter with Variable

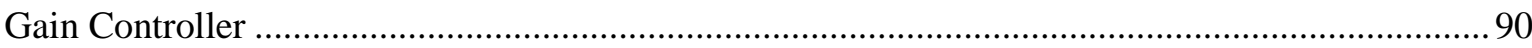

Figure 4-13 - Open Loop Bode Plot of Voltage Loop for Buck Converter................................... 91

Figure 4-14 - Buck Converter with Bootstrap Driver and Synchronous Switch [32] ..................... 93

Figure 4-15 - Synchronous PulseStart to charge Bootstrap Capacitor ............................................. 95

Figure 4-16 - Buck Synchronous Pulse Start Waveform ............................................................ 96

Figure 4-17 - Photo of Prototype of Buck Maximum Energy Extraction and Charge Controller.... 98

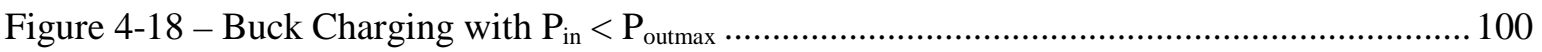

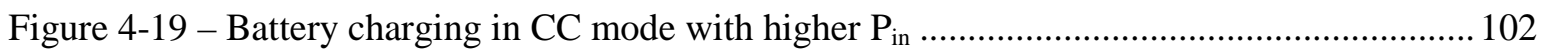

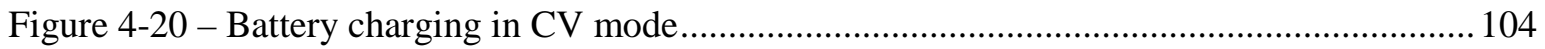

Figure 4-21 - DC Efficiency of Buck Converter in CC Mode ................................................... 105

Figure 5-1 - ADC Sampling and Interrupt Service Routine Timing Diagram ............................... 111

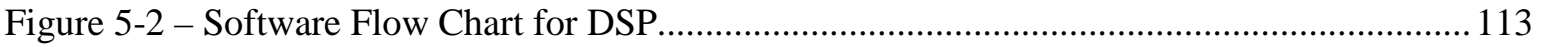

Figure 5-3 - Selection guide for $\mathrm{V}_{\text {boostmax }}$ vs. Energy Storage Capacitor size with Pout $=12.8$ watts

Figure 5-4 - Full system operation, $\mathrm{R}_{\text {in }}=15 \Omega$, Buck in CC mode ............................................. 120

Figure 5-5 - Full System operation, $\mathrm{R}_{\text {in }}=15 \Omega$, Buck in CV mode ............................................. 121

Figure 5-6 - Mechanical Test Rig with DC motor drive on the left and 3-phase generator shown on

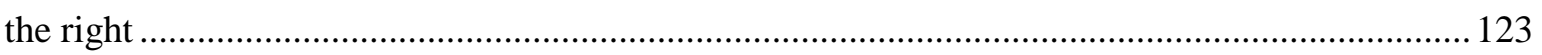

Figure 5-7 - Electrical Schematic of Mechanical Test Rig ...................................................... 124

Figure 5-8 - PEM excited by Mechanical Test Rig.................................................................... 125

Figure 5-9 - Single Stage Buck excited by Mechanical Test Rig............................................... 126

Figure 5-10 - Complete System DC Efficiency................................................................... 127

Figure 5-11 - PEM System Efficiency with AC Input ............................................................ 128 


\section{List of Tables}

Table 2-1 - Comparison between Conventional PFC and proposed $\mathrm{R}_{\text {in }}$ Controlled Boost Converter . .28

Table 3-1 - Table of Components used in Boost Converter ............................................................. 44

Table 3-2 - Table of Values used for Voltage Dividers .................................................................... 46

Table 3-3 - Quantized Values for Voltage Adaptive Gain ............................................................ 61

Table 4-1 - Table of Components used in Buck Converter .............................................................97

Table 5-1 - Delay of Sampled Signals from Sampling Instant to when they take Effect............... 115 


\section{List of Symbols}

Ai

$\mathrm{Bi}$

C

$\mathrm{C}_{\text {Boost }}$

$\mathrm{C}_{\text {bootstrap }}$

$\mathrm{C}_{\text {Buck }}$

$\mathrm{C}_{\mathrm{ESC}}$

$\hat{d}$

D

$\mathrm{D}_{\max }$

$\mathrm{E}$

$\mathrm{E}(\mathrm{n})$

$\mathrm{F}_{\text {in }}$

$\mathrm{f}_{\mathrm{sW}}$

$\mathrm{G}_{\text {adp }}$

$\mathrm{G}_{\mathrm{ciBuck}}(\mathrm{z})$

$\mathrm{G}_{\mathrm{cvBuck}}(\mathrm{z})$

$\mathrm{G}_{\mathrm{cBoost}}(\mathrm{z})$

$\mathrm{G}_{\mathrm{i}}(\mathrm{s})$

$\mathrm{G}_{\mathrm{iBuck}}(\mathrm{s})$

$\mathrm{G}_{\mathrm{P}}$

$\mathrm{G}_{\mathrm{pBoost}}(\mathrm{s})$
Digital controller pole coefficient $i,(i=1,2)$

Digital controller zero coefficient $i,(i=0,1,2)$

Capacitor

Boost Capacitor

Bootstrap Capacitor

Buck Capacitor

Energy Storage Capacitor

Small signal duty cycle

Steady-state duty cycle

Maximum duty cycle

Energy in Joules

Error Signal

Input frequency from generator (AC envelope)

Switching Frequency

Voltage Adaptive Compensator Gain

Discrete-time compensator of Buck converter current loop

Discrete-time compensator of Buck converter voltage loop

Digital Boost compensator

Current loop compensator

Small signal transfer function of Buck converter from duty cycle to inductor

current

Gain of P-Controller

Small signal power stage model of average current mode Boost converter 


\begin{tabular}{|c|c|}
\hline $\mathrm{G}_{\mathrm{pBoost}}(\mathrm{z})$ & Discrete-time transfer function of Boost converter including digitization effects \\
\hline $\mathrm{G}_{\mathrm{vBuck}}(\mathrm{s})$ & Small signal power stage model from inductor current to output voltage for Buck \\
\hline & converter \\
\hline $\mathrm{G}_{\mathrm{v}}(\mathrm{s})$ & Voltage loop compensator \\
\hline $\mathrm{H}_{\mathrm{c}}$ & Computational Delay Block \\
\hline $\mathrm{I}_{\text {Boost }}$ & Boost inductor current \\
\hline $\mathrm{I}_{\text {Buck }}$ & Buck inductor current \\
\hline $\mathrm{I}_{\mathrm{CC}}$ & Constant Current charging rate of battery \\
\hline $\mathrm{I}_{\text {CCMAX }}$ & Maximum Constant charging rate of battery \\
\hline $\mathrm{I}_{\text {charge }}$ & Charging Current of battery \\
\hline $\mathrm{I}_{\text {in }}$ & Input current drawn from generator \\
\hline$\hat{i}_{l}$ & Small signal inductor current \\
\hline $\mathrm{I}_{\mathrm{L}}$ & Steady-state inductor current \\
\hline $\mathrm{I}_{\mathrm{L}}(\mathrm{n})$ & Digital representation of sampled inductor current \\
\hline $\mathrm{I}_{\text {ref }}$ & Current reference signal \\
\hline $\mathrm{K}_{\mathrm{d}}$ & Sensing network gain \\
\hline $\mathrm{K}_{\mathrm{i}}$ & Gain of current sensing network for Buck Converter \\
\hline $\mathrm{K}_{\mathrm{v}}$ & Gain of voltage sensing network for Buck Converter \\
\hline $\mathrm{L}_{\text {Boost }}$ & Boost Inductor \\
\hline $\mathrm{L}_{\text {Buck }}$ & Buck Inductor \\
\hline $\mathrm{n}$ & Current sampling cycle value \\
\hline$n-1$ & One sample cycle old value \\
\hline $\mathrm{N}_{\text {cells }}$ & Number of cells in battery pack \\
\hline $\mathrm{P}_{\mathrm{avg}}$ & Average power of PEM \\
\hline
\end{tabular}




\begin{tabular}{|c|c|}
\hline $\mathrm{P}_{\text {battmin }}$ & Minimum of the maximum battery charging power \\
\hline $\mathrm{P}_{\text {gen-avg }}$ & Average power generated by the human energy harvester \\
\hline$P_{\text {in }}$ & Input power generated by the human energy harvester \\
\hline$P_{\text {inmax }}$ & Peak input power \\
\hline $\mathrm{P}_{\text {out }}$ & Output power to the battery \\
\hline$P_{\text {outmax }}$ & Peak output power to the battery \\
\hline $\mathrm{Q}_{1}$ & Control MOSFET \\
\hline $\mathrm{Q}_{2}$ & Synchronous MOSFET \\
\hline $\mathrm{R}_{\text {batt }}$ & Internal resistance of the battery \\
\hline $\mathrm{R}_{\mathrm{ext}}$ & External resistor added to DC motor \\
\hline $\mathrm{R}_{\text {in }}$ & Emulated input resistor value \\
\hline$r_{\text {ind }}$ & Inductor series resistance \\
\hline $\mathrm{R}_{\mathrm{L}}$ & Load resistor \\
\hline $\mathrm{R}_{\text {sense }}$ & Current sense resistor \\
\hline $\mathrm{s}$ & Laplace transform variable \\
\hline $\mathrm{T}_{\mathrm{d}}$ & Computational delay \\
\hline $\mathrm{T}_{\mathrm{s}}$ & Sampling period \\
\hline $\mathrm{U}(\mathrm{n})$ & Digital controller output \\
\hline V & Volts \\
\hline $\mathrm{V}_{\mathrm{ADCmax}}$ & Maximum voltage that can be sensed by the ADC \\
\hline$\hat{v}_{\text {batt }}$ & Small signal battery voltage \\
\hline $\mathrm{V}_{\text {batt }}$ & Battery voltage \\
\hline$\hat{v}_{\text {Boost }}$ & Small signal output voltage of the Boost converter \\
\hline
\end{tabular}




$\begin{array}{ll}\mathrm{V}_{\text {Boost }} & \text { Output voltage of the Boost converter, voltage of the energy storage capacitor, } \\ & \text { input voltage of the Buck converter } \\ \mathrm{V}_{\text {Boostmax }} & \text { Maximum voltage of } \mathrm{V}_{\text {boost }} \\ \mathrm{V}_{\text {Boostmin }} & \text { Minimum voltage of } \mathrm{V}_{\text {boost }} \\ \mathrm{V}_{\text {Buck }} & \text { Output voltage of the Buck converter } \\ \mathrm{V}_{\text {cvmax }} & \text { Maximum voltage of the battery } \\ \mathrm{V}_{\text {in }} & \text { Input voltage generated by the generator } \\ \mathrm{V}_{\text {l-1 }} & \text { Line to line voltage of generator } \\ \mathrm{V}_{\text {min }} & \text { Minimum voltage of lithium polymer cell } \\ \mathrm{V}_{\text {ref }} & \text { Reference voltage } \\ \mathrm{V}_{\text {sample }} & \text { Voltage of the signal to be sampled } \\ \hat{V}_{o} & \text { Small signal output voltage } \\ \mathrm{V}_{\text {rect }} & \text { Rectified input voltage } \\ \hat{V}_{\text {rect }} & \text { Small signal input voltage } \\ \mathrm{Z} & \text { Z-transform variable }\end{array}$




\section{List of Abbreviations}

AC

ADC

CC

CCM

$\mathrm{COH}$

CSA

CV

DC

DDHO

DSP

EDLC

EoS

ePWM

ESC

ISR

MEECC

MOSFET

PCB

PEM

PFC

PVDF

SEPIC

SoC
Alternating Current

Analog to Digital Converter

Constant Current

Continuous Conduction Mode

Cost of Harvesting

Current Sense Amplifier

Constant Voltage

Direct Current

Driven Damped Harmonic Oscillator

Digital Signal Processor

Electrochemical Double Layer Capacitor

End of Sequence

Enhanced Pulse Width Modulation

Energy Storage Capacitor

Interrupt Service Routine

Maximum Energy Extraction and Charge Controller

Metal Oxide Semiconducting Field Effect Transistor

Printed Circuit Board

Power Electronics Module

Power Factor Correction

Piezoelectric Polymer Polyvinylidene Fluoride

Single Ended Primary Inductor Converter

State of Charge 
$\mathrm{ZOH}$

Zero-order Hold 


\section{SI Units}

$\begin{array}{ll}\text { A } & \text { Amperes } \\ \mathrm{F} & \text { Farads } \\ \mathrm{H} & \text { Henries } \\ \mathrm{Hz} & \text { Hertz } \\ \mathrm{J} & \text { Joules } \\ \mathrm{M} & \text { Meters } \\ \mathrm{s} & \text { seconds } \\ \mathrm{V} & \text { Volts } \\ \mathrm{W} & \text { Watts } \\ \Omega & \text { Ohm }\end{array}$

\section{Prefixes for SI Units}

$\begin{array}{ll}\mathrm{p} & \text { Pico }\left(10^{-12}\right) \\ \mathrm{n} & \text { Nano }\left(10^{-9}\right) \\ \mu & \text { Micro }\left(10^{-6}\right) \\ \mathrm{m} & \text { Milli }\left(10^{-3}\right) \\ \mathrm{k} & \text { Kilo }\left(10^{3}\right) \\ \mathrm{M} & \text { Mega }\left(10^{6}\right)\end{array}$

xvii 


\section{Chapter 1}

\section{Introduction}

\subsection{Introduction to Human Energy Harvesting}

We are well on our way into a new phase of the information age as humans are becoming more dependent on portable personal electronic devices. The number of people using multiple of these electronic devices on a daily basis is ever increasing. These devices can range from medical devices such as pacemakers, electromechanical or neuroelectric prosthesis to consumer devices such as cellular phones, smart phones, digital music players, global positioning systems, personal digital assistants, and tablets. The military also uses a wide range of portable electronics such as night vision goggles, communication devices, flashlights, laser range finders, and many more. The one thing that all of these electronic devices have in common is that they are all powered by batteries. Unfortunately the speed in which new and more powerful electronics are created is not met with the speed in advancement of the battery technology that is used to power these devices. The increasing power requirements of the devices mean that the increasing battery size, weight and charging time are becoming inconvenient for the users. In the past few years there has been increasing interest in the indirect scavenging of energy from everyday human motion or actions, in order to reduce the dependence on larger sized batteries or remove batteries from the system all together[1]. This biomechanical energy harvesting would take place such that the extraction of power is practically transparent to the user. An example of a device that uses human energy harvesting for its everyday operation is the self-winding watch, the Seiko AGS, in which the everyday movement of the user's arm causes a proof mass to rotate and can generate $5 \mu \mathrm{W}$ of power on average[2]. The challenge 
with human energy scavenging often is to produce significant power without making the everyday actions too cumbersome for the user.

Such an energy harvesting device could reduce the burden of short runtime, size and weight that batteries inherently introduce. The energy from the human energy harvester would also be available when the utility grid is down or not nearby, thus the electronic devices could be recharged during a blackout or in remote locations.

Much effort has gone into the design of human energy harvesting devices. Unfortunately, the same is not true for the power electronic circuitry that is needed to store and make full use of this power. Many of the existing designs do not have much more than a simple resistor to measure the output power of their devices and the designs that do have power electronics are oversized to simplify the task.

\subsection{Previous Works on Human Energy Harvesting Generator Designs and Power Electronics Used}

This section will review the existing technology present to address the design of human energy harvesting generators as well as their power electronic circuitry used to store the power generated.

\subsubsection{Human Energy Harvesting Generator Designs}

There have been numerous approaches to address the design of human energy harvesters, with the average power ranging from the $\mu \mathrm{W}$ level [3], to the $\sim 18 \mathrm{~W}$ level[4]. There are generators consisting of shoe-mounted designs [5-9], with one generator using an electro active polymer that can produce up to $0.8 \mathrm{~W}$ average by the deflection of the material caused from walking[8]. A linear permanent magnet motor shoe apparatus has been reported to harvest energy from the linear motion 
of the foot during walking and with its maximum power point tracking algorithm it can produce as much as $0.83 \mathrm{~W}$ average[9].

Another method of energy harvesting has been proposed in $[10,11]$ that produces energy from the joint movement of the knee during normal walking shown in Figure 1-1. It was reported that this method of generation could produce as much as $7 \mathrm{~W}$ of average power when operated in continuous conduction mode and $4.8 \mathrm{~W}$ of average power when the generator is selectively engaged to harvest only negative power produced by the knee, called the generative breaking mode [11]. This generative breaking mode was able to produce power with a cost of harvesting $(\mathrm{COH})$ value of 0.7 . This means that for every extra watt of power that was generated the user only needed to output an extra 0.7 watts of metabolic energy [10].
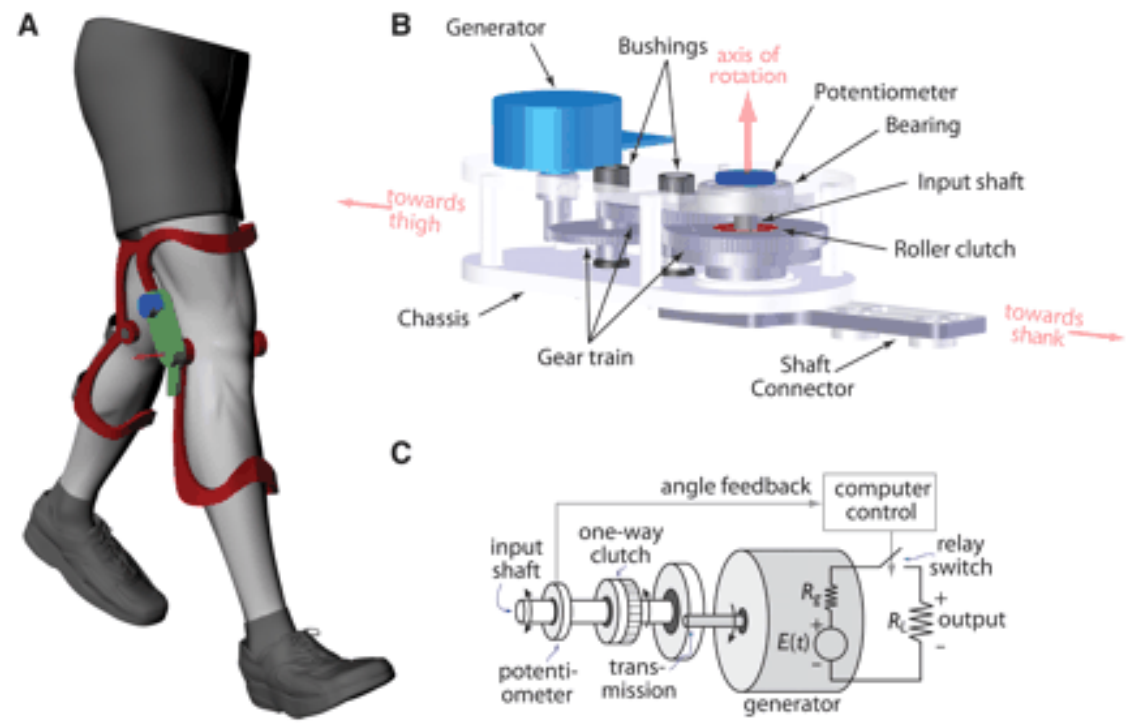

C

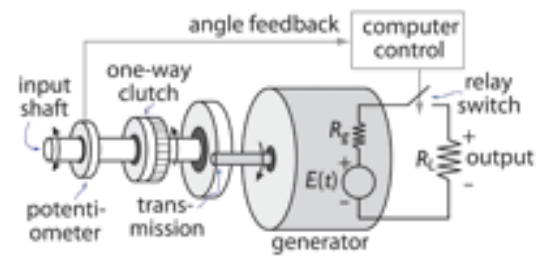

Figure 1-1 - Biomechanical Knee Brace Energy Harvester [11] 
One of the more common types of human energy harvester that has recieved a lot of interest lately is backpack based energy harvesters [4, 12-15]. The backpack based generators provide a convenient location for mounting the generator and the weight of the generator apparatus is close to the center of mass of the body. The first type of backpack generator design is one that uses piezoelectric polymer polyvinylidene fluoride (PVDF) straps to generate electricity based on the differential forces between the backpack and wearer of the backpack [12]. However, this type of energy generation was only able to produce $45.6 \mathrm{~mW}$ of average power with a $45 \mathrm{~kg}$ backpack load.

More power can be produced when using a driven damped harmonic oscillator (DDHO) type of backpack generator, in which the backpack's mass is connected to the frame of the backpack through a spring system allowing the mass to oscillate. When the wearer of the backpack walks, it excites the mass and spring system causing linear displacement between the backpack mass and backpack frame. These types of generators can convert the linear motion of the backpack mass with respect to the backpack’s frame into electrical energy either through a linear motor [14] or through a rotary-magnetic generator $[4,13]$. The rotary-magnetic generator, shown in Figure 1-2, converts the linear motion of the backpack into rotational motion through the use of a rack and pinion gear system, and the rotational motion is converted to electrical energy through the use of a generator. 


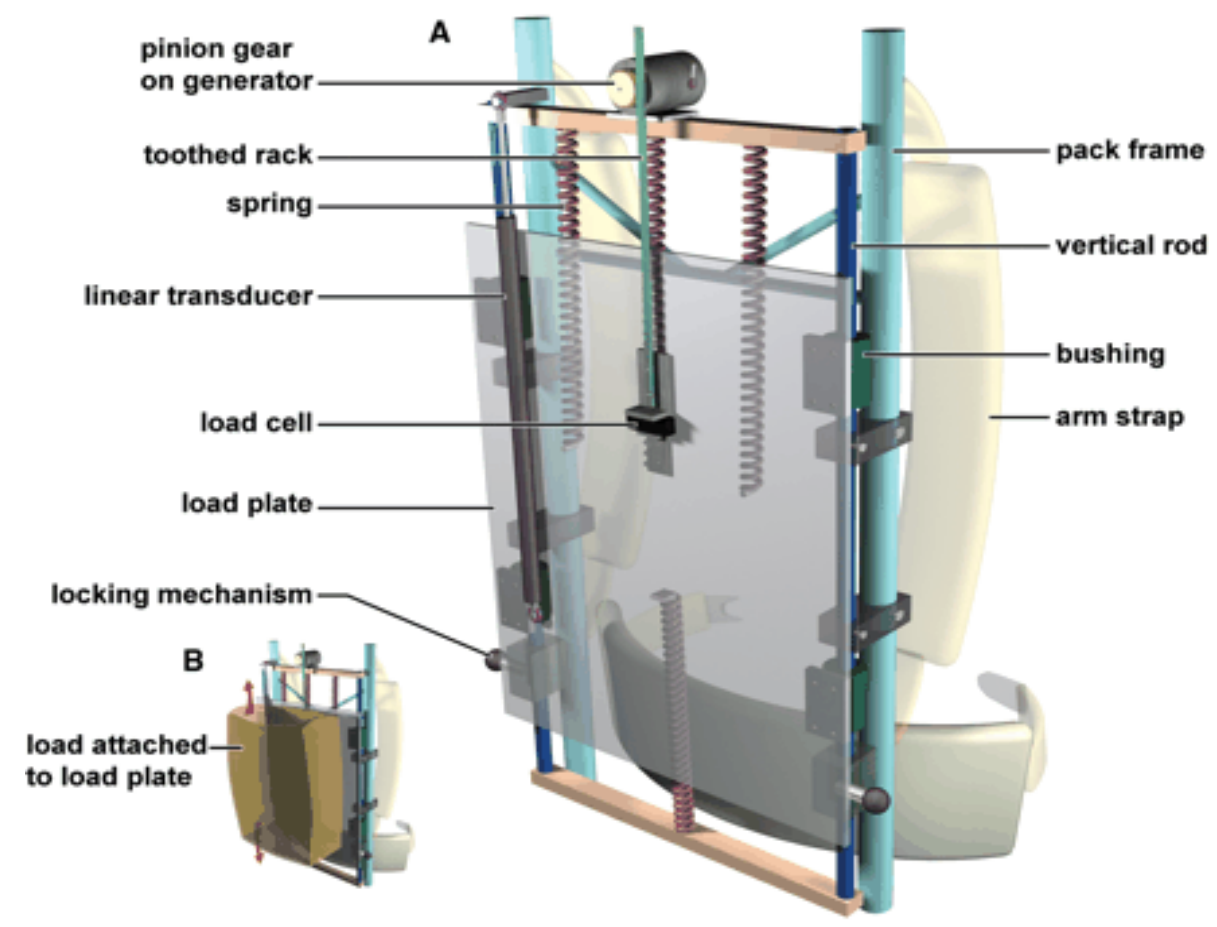

Figure 1-2 - Backpack Based Linear Motion Energy Harvesting System [13]

Experimental tests conducted with this type of generator have produced as much as 18 watts of average power while being excited by a mechanical test bed [4]. A backpack design with the advantages of the knee brace to harvest energy from the walking motion of a human is currently being developed by our collaborators in the mechanical engineering department. This generator will require a new power electronics system that is capable of extracting power from the novel generator according to the user's desired input power profile and storing all of the extracted energy into an appropriately sized battery while staying within the charging limitations of the battery.

\subsubsection{Power Electronic Circuitry used in Human Energy Harvesting}

From this, it can be seen that there are numerous methods of generating electricity from everyday human motions. These generators are reported to produce average power anywhere from the $\mu \mathrm{W}$ range, [3] to the $18 \mathrm{~W}$ level [4]. However, the majority of the work mainly focused on the generator 
design and has left the design of electronic circuitry necessary to make full use of this power out. They are simply using a resistor bank to measure the output power of their devices and all the energy that is generated is lost $[6-8,10-13,15]$. A few of the energy harvesters mentioned do, however, have the design of the power electronic circuitry that is needed to store the harvested energy $[4,9,14,16]$. Although the application of the power electronic circuitry will change depending on the type of generator it is connected to, it is still worth looking into the different designs and control techniques used.

The authors in [16] have created a mini-vibrational power generator that is capable of generating 35 $\mathrm{mW}$ average power. In their power electronic circuit, they have used a diode bridge to rectify the input voltage followed by a Boost converter to step up the voltage to the output voltage level of the super-capacitor storage medium. Their circuit schematic is shown in Figure 1-3.

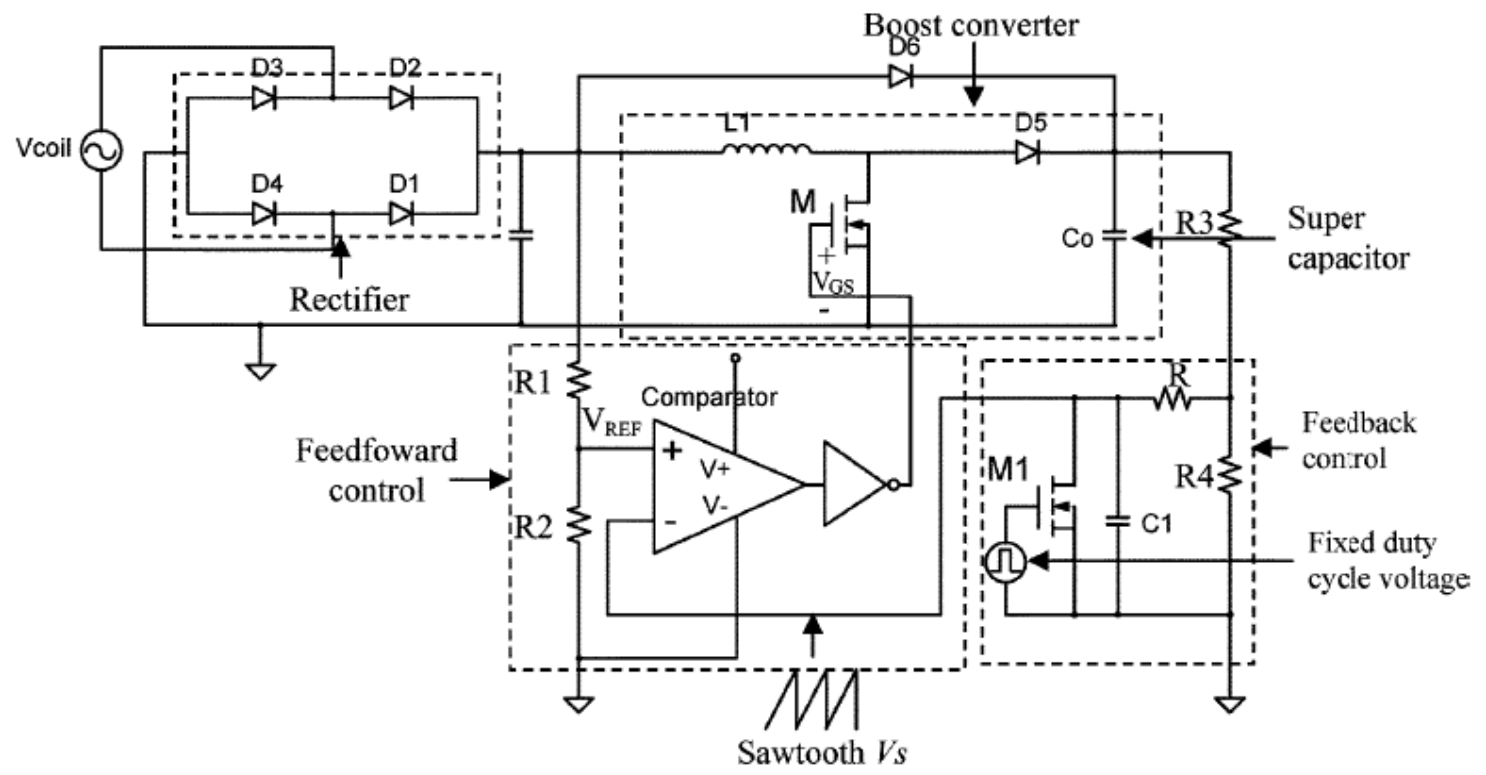

Figure 1-3 - Mini-Vibrational Power Generator Circuit Schematic [16] 
The advantage of this circuit is that it is able to extract power when the input voltage is less than the voltage of the energy storage medium. The main disadvantage of this circuit is that the energy storage element used is a super-capacitor. The specific energy density of a super-capacitor is low and only a fraction of that of a battery. The amount of capacitance required to store a moderate amount of energy would be quite large. Super-capacitors also have a high self-discharge rate when compared to rechargeable batteries, meaning that the power generated could not be stored for a long period of time[17]. If the power that was generated was not used soon after it was generated, much of it would be lost. As the primary function of this circuit is to provide power for a micro sensor network, the power requirements are quite low and the super-capacitor would likely be a reasonable choice for the energy storage element because the power would be used as soon as it was generated[16]. The average power level of $35 \mathrm{~mW}$ is too low to be worth the complexity that a battery and charging circuit would introduce into the design.

As the power level of the generator increases, the design of the power electronic circuitry becomes more important. The next two power circuit topologies that will be reviewed both have in common that they are conditioning the power generated from an oscillating spring mass backpack system. It should be noted that in this application the power electronic circuitry provides linear damping of the mechanical dynamics of the backpack through resistive emulation on the terminals of the generator. This damping must always be present during excitation of the mechanical system in order to prevent excessive displacements of the mass in the backpack, which would occur in an under damped system. This presents a unique challenge for the power electronic circuitry. If the battery cannot absorb all of the power that is being generated, the circuit must dump the energy that was harvested through a resistor bank, as was presented in [4]. 
The power electronic circuit that was used in [4] consists of a diode rectifier followed by a resistance-emulating SEPIC Converter, shown in Figure 1-4. The output of the SEPIC converter is connected to the output bus that connects to a switchable resistive load, an ultra-capacitor bank for energy storage, a lithium-ion battery pack connected through a diode and a voltage regulator to provide power for external electric loads.

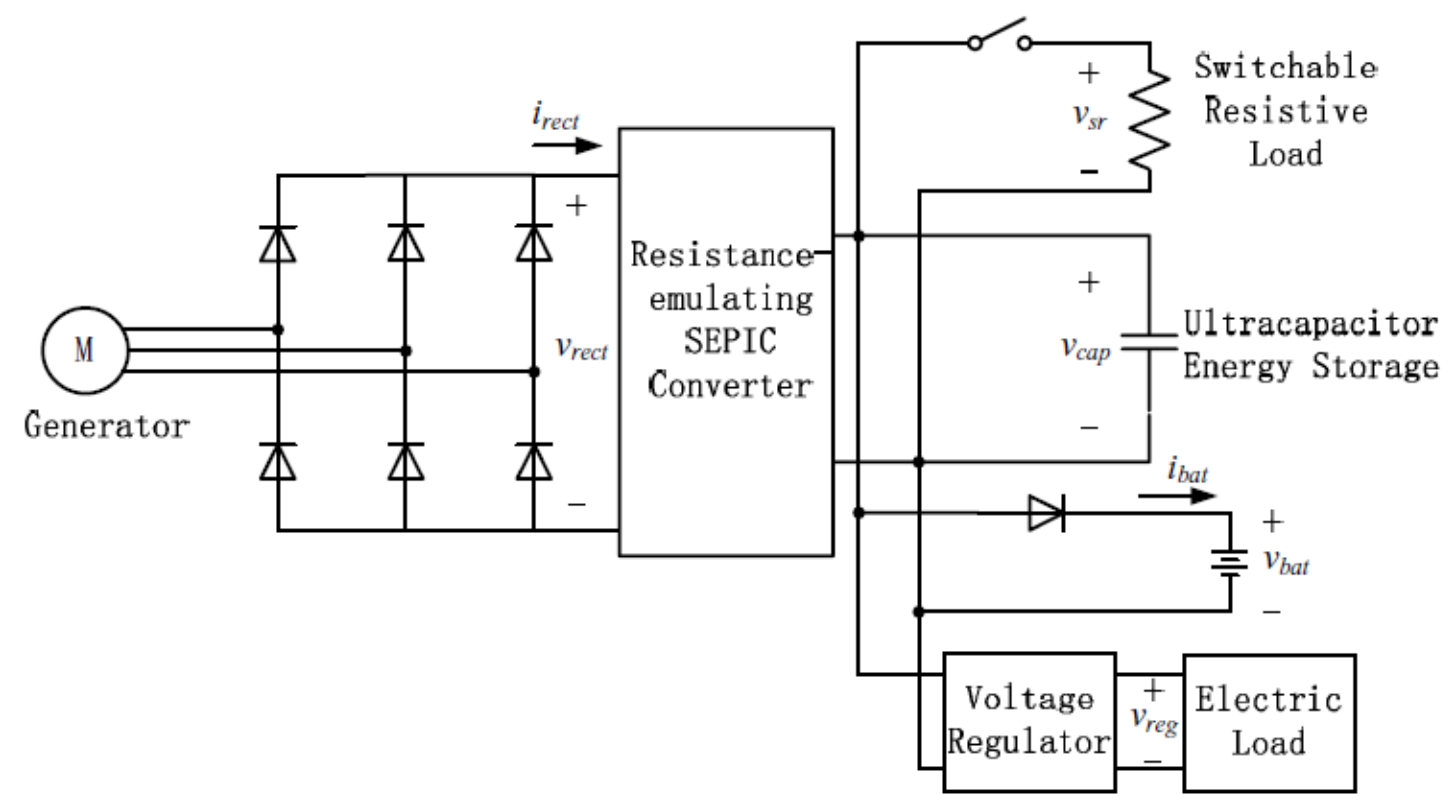

Figure 1-4 - Schematic of Mechanical Oscillator Energy Harvester Backpack System [4]

The regulation of the voltage of the battery pack is achieved through the use of the diode and switchable resistive load bank. When the output bus voltage rises to a level that would cause the battery voltage to go over the voltage limit, the switchable resistive load is switched on to dissipate the excess power that cannot be stored. This causes the output voltage of the ultra-capacitor storage bank to decrease, which also reduces the charging voltage of the battery. Once the voltage has decreased below a certain threshold, the switchable load will turn off. The problem with this 
method is that there is no direct control of the charging voltage or the charging current to the battery. The regulation of the charging current comes from the fact that the battery pack maximum charging power is much higher than the peak power capabilities of the generator. The maximum charging power of the BB-2590 Bren-Tronics battery pack that was used in the design [4] is 152 225 watts based on a $1 \mathrm{C}$ charge rate[18]. The peak power produced by the generator was measured at 50 watts, and after filtering of the power by the 5 Farad 16.2 Volt ultra-capacitor energy storage bank the peak charging power of the battery was reduced to 32 watts with the average charging power of the battery equal to 17 watts. From the power ratings, we can conclude that the size of the battery is grossly oversized for the application. As the peak power level generated by the energy harvester is much less then the peak charging power of the batteries, the batteries will never reach the charging current limitation of 13.6A

If the battery was charged from empty to full based on the average power level of the backpack, it would take more than 12 hours of walking to charge at full power. Although the oversized battery pack simplifies the control needed in the power electronic circuit it is not ideal as the larger battery pack is required as part of the design to ensure that the battery cannot go over the charging current limitation. This overdesign of the battery pack will increase the cost of the converter as well as the weight of the total system. The ultra-capacitor energy storage bank is also not ideal, as the bank of capacitors is not fully utilized as can be observed in the voltage waveform of the battery, shown in Figure 1-5. 

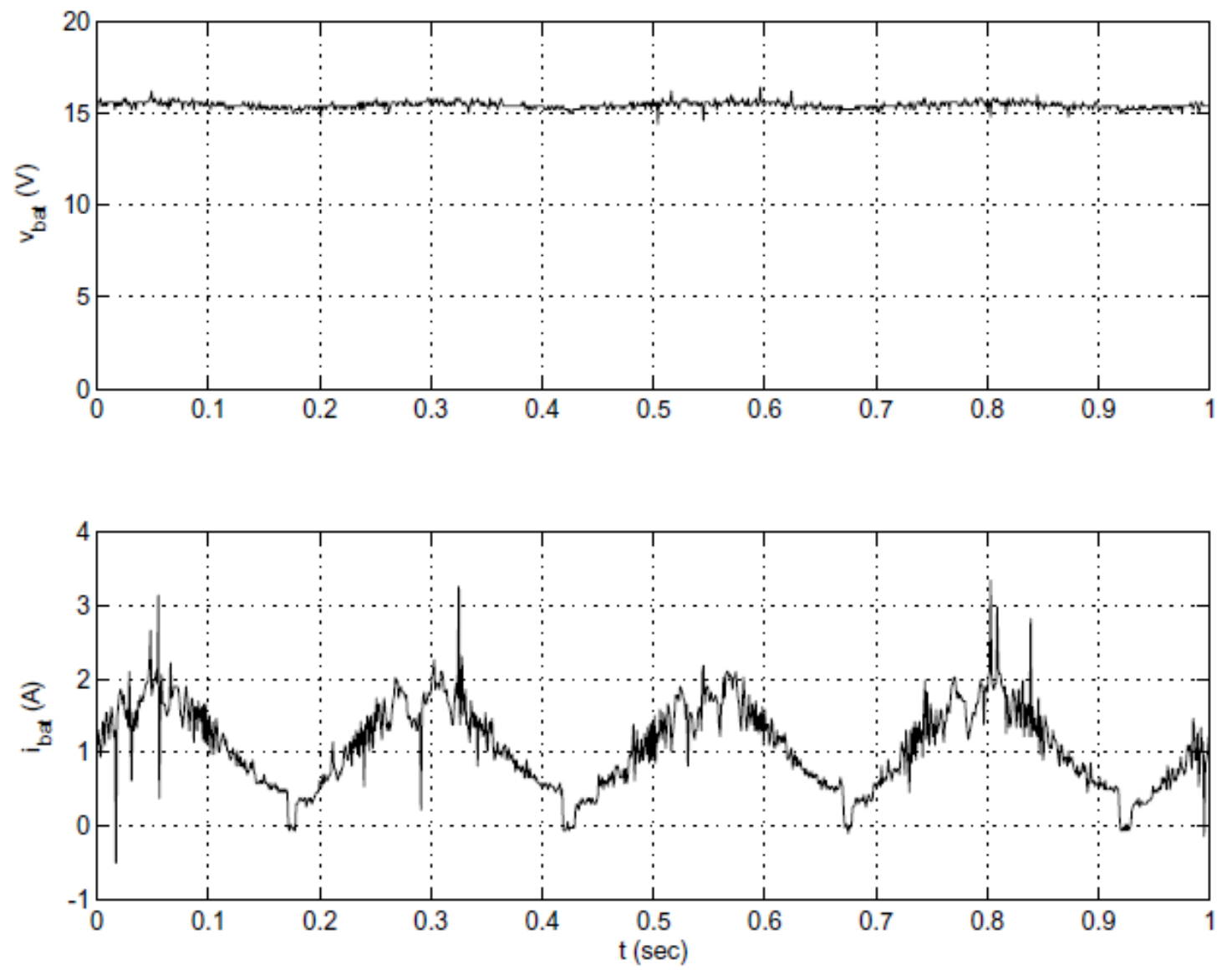

Figure 1-5 - Mechanical Oscillator Energy Harvester Backpack System Battery Charging Voltage and Current waveforms [4]

The voltage of the ultra-capacitor storage bank can be inferred from the battery voltage waveform from the figure. It is approximately 0.5 Volts higher than the battery voltage, due to the Schottky diode in series with the battery. From this we can see that in normal operation the voltage swing of the ultra-capacitor energy storage bank is approximately $16.2 \mathrm{~V}-15.7 \mathrm{~V}$. The total energy that is stored by the 5 Farad capacitor bank can be calculated using (1.1), where $V_{2}$ is the maximum voltage stored in the capacitor and $\mathrm{V}_{1}$ is the minimum voltage stored in the capacitor.

$$
E=\frac{1}{2} C\left(V_{2}^{2}-V_{1}^{2}\right)
$$


The energy stored in the capacitor bank during this voltage swing is 40 Joules. The total energy capability of the capacitor bank can be found by setting the minimum voltage of the capacitor, $\mathrm{V}_{1}$, to $0 \mathrm{~V}$. The total energy capability of the capacitors is 656 Joules of energy with only $6 \%$ of the total energy storage capability used. Much of the capacity cannot be used because the voltage level of the capacitor bank is clamped through the diode to the voltage of the battery pack.

The power electronics system used in [14] is somewhat similar to that used in [4] and is shown in Figure 1-6. The voltage of the generator is rectified by the diode rectifier and connected to a Boost converter. The output of the Boost converter is connected to a $24 \mathrm{~V}$ battery for energy storage.

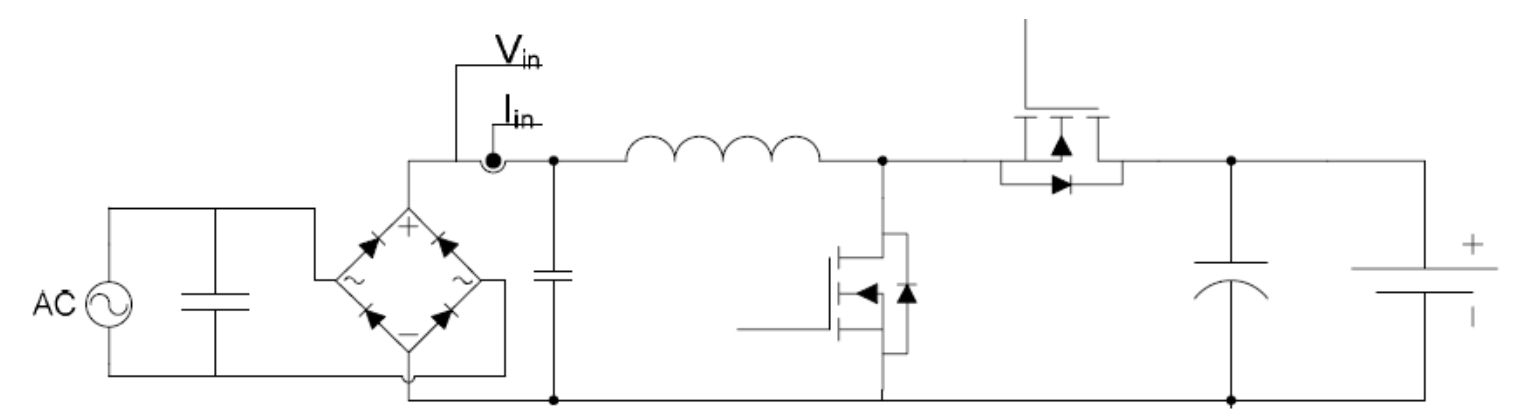

\section{Figure 1-6 - Power Electronic Circuit used in [14]}

We can infer from the circuit design that without a form of immediate energy storage, such as an energy storage capacitor, the battery must be absorbing the full amount of the time varying input power that would be generated by the mechanical mass oscillator system. It must be assumed that the battery is of significant capacity in order for it to safely charge at the peak power level of the time varying input power. In this design, the main concern is with the extraction of power from the generator while emulating the maximum power point resistor value, with little consideration into the regulation of the charging current and charging voltage of the battery. It must be assumed that, with a battery of large capacity, charge regulation was achieved because the input power was less 
than that of the battery charging power and there was no need to worry about regulating the charging conditions of the battery similar to the previous design.

\subsection{Research Motivation}

The power electronic circuitry is a crucial part of the energy harvesting process. The limitations of existing energy harvester's power electronics circuits are emphasized, providing the motivation for this thesis.

\subsubsection{Maximize the Amount of Power Extracted from the Generator While Controlling the Input Power}

The majority of human energy harvesters do not have power electronic circuits required to store the extracted energy. The generators with power electronics that produce significant power are all based on a mass and spring oscillator backpack design. The primary function of the power electronics in these designs is to provide adequate damping for the oscillating system while extracting power. This damping must always be present to prevent the mass system from excessive displacements. The power level of these devices has a limited operating range and power must always be extracted from the system for proper operation. They also require a large amount of user energy to wear the backpack, regardless if the energy is being extracted or not, because of the large mass of the generator.

A power converter is needed that can maximize the power output of the generator based on the user's desired effort level. The power converter will only draw power from the generator if it can be used or stored. 


\subsubsection{Store Extracted Power into Batteries While Not Exceeding the Charging Limitations of an Appropriately Sized Lithium Polymer Battery}

The human energy harvesters that do have power electronics and store the energy in a battery all have a similar design flaw, they all require large batteries. The input power from an energy harvesting generator is varying with the frequency of a human gait cycle, usually $1-2 \mathrm{~Hz}$. The peak power generated can be as much as five times the average power produced by the generators. As the instantaneous input energy at this peak power level must be stored in the batteries, the batteries must be of significant capacity that they can be charged safely at this power level.

One of the designs uses an ultra-capacitor to reduce the peak power from $50 \mathrm{~W}$ to $32 \mathrm{~W}[4]$. However, it requires a capacity of 5 Farads to do so. To further simplify the design there is no controller to monitor the charging current of the batteries. The need for a charge regulator was eliminated by further increasing the battery size such that under normal operating conditions, the output power of the generator will never exceed the maximum charging power of the battery. This creates the problem that for a given power level, a minimum size of battery is required. This will inherently increase the cost, size and weight of the generator.

A power converter is needed that can temporarily store the peak power produced by the generator, such that the battery size can be reduced. A controller needs to be designed that can regulate the charging conditions of the smaller battery while storing the excess energy that is caused from the time varying input power profile. The battery size can be reduced such that it only needs capacity to store the average power that is generated, which is much lower than the peak power generated. 


\subsection{Thesis Objectives}

The objective of this thesis are: 1 ) to design a power converter that can extract the maximum power from the novel generator according to the user's desired input power profile, and 2) store all of the extracted energy into an appropriately sized battery while staying within the charging limitations of the battery.

The first objective will allow the power converter to extract current from the generator in any desired current profile, which will allow the power profile to be adjusted to reduce the user's energy input for power generation. Power will only be extracted from the generator when it can be either used or stored, reducing the energy cost of the user. The second objective will allow the converter to use a smaller capacity battery chosen based on the average power generated as opposed to the peak power generated, reducing the required capacity of the battery for a given application. This will reduce the cost, size and weight of the total system.

\subsection{Thesis Organization}

Chapter 1 introduces the subject of human energy harvesting and summarizes the many different variations of energy harvesting devices and power ratings of the generators. The power electronics that are used to store the harvested energy are analyzed and the shortcomings of the designs are identified. It establishes motivation and sets out the objectives for the research contributions presented in this thesis.

Chapter 2 proposes a power electronic system diagram that is designed to overcome the shortcomings of the previous designs while meeting the design requirements of a novel generator designed by collaborators in the mechanical engineering department. The design identifies what the 
controllers must accomplish and how the energy storage capacitor is used to reduce the size of battery needed while not sacrificing potential energy harvest.

Chapter 3 introduces the control strategy used to control the Boost converter to achieve the design objective of extracting the maximum power from the novel generator according to the user's desired input power profile. The functions of the three operating modes of the controller are discussed. The transfer function of the Boost average current mode control is derived and transformed into the Z-domain adding the effects of digitization into the control loop. A digitally controlled Boost converter prototype is designed and experimental results are presented to test the controller's functionality and validate that the design can meet the thesis objectives. A Voltage Adaptive Gain compensator is designed to help achieve better dynamic performance over the wide output voltage range of the Boost converter. The DC efficiency of the Boost converter is measured for different power input levels.

Chapter 4 introduces the Maximum Energy Extraction and Charge Controller that was used to meet the second objective of the thesis, which is, to store all of the extracted energy into an appropriately sized battery while staying within the charging limitations of the battery. The MEECC controller design is discussed along with the operation of the four sub-controllers that make up the MEECC controller. The transfer function of the voltage loop and current loop are derived and a Voltage Adaptive Gain compensator is designed to help achieve better dynamic performance over the wide input voltage range of the Buck converter. A method of driving the synchronous Buck converter without voltage isolation of the driver is presented. A digitally controlled Buck converter prototype is designed and built with experimental results to show the effectiveness of the MEECC controller in meeting the second objective of the thesis. 
Chapter 5 discusses the design process for the sections of the power electronics module that are not covered in Chapters 3 and 4 . Namely the considerations of the embedded software on the DSP, a software flow chart, and a design guide for the selection of the energy storage capacitor size and voltage rating. Experimental results are presented to verify the complete system and controllers can work in unison to meet the combined objectives of the thesis. Verification that the design of the two stage converter with energy storage capability can extract more power than a single stage design without energy storage capability is conducted using a mechanical test rig.

Chapter 6 summarizes the contributions of the research presented in this thesis and gives suggestions for future work. 


\section{Chapter 2}

\section{Application}

\subsection{Introduction}

As the need for such portable human powered generators has become apparent in the previous chapter, a novel generator is currently been developed by our collaborators in the mechanical engineering department. This chapter will present the power electronics design that is required to meet the design objectives of this thesis: 1) Design a power converter that can extract the maximum power from the novel generator according to the user's desired input power profile. 2) Store all of the extracted energy into an appropriately sized battery while staying within the charging limitations of the battery.

\subsection{Application}

The generator will generate electricity using the walking motion of a human, known as the user, as the input energy source. The power generated is in a form such that it needs to be conditioned and converted in order for it to be effectively harvested for use in charging portable electronics. The power electronics system that is required to do this task will be referred to as the Power Electronics Module (PEM) and will have the following design requirements.

The PEM will need to convert the output waveform of the three-phase Alternating Current (AC) generator of the energy harvester into a Direct Current (DC) waveform that is suitable for charging batteries. The PEM must also control the amount of current drawn from the generator into any specified profile as determined by the user. This will allow power to be extracted from the energy harvester at specific points along the user's gait cycle as well as to control how much energy is 
extracted during the gait in order to ensure that the amount extracted is not too burdensome for the user. This power input curve can later be optimized by the bio-mechanical engineers in order to extract the most amount of power with minimal user effort in order to achieve a low Cost of Harvesting $(\mathrm{COH})$ as was demonstrated in [10]. As any energy that is extracted from the generator potentially comes at a ,metabolic cost to the user, it is a requirement that any energy extracted must be effectively stored in the batteries and not simply 'wasted' because there is no capacity to store it.

The typical voltage waveform from the output of the generator is a variable high frequency threephase voltage inside a lower frequency $(1-2 \mathrm{~Hz})$ AC envelope similar to the waveform shown in Figure 2-1. The frequency of the outside AC envelope is dependent on the user, walking speed and frequency of the user's gait cycle. Figure 2-1 is a typical waveform seen of a user walking at a normal speed of $1.5 \mathrm{~m} / \mathrm{s}(5.4 \mathrm{~km} / \mathrm{h})$. The frequency of the variable high frequency is also dependent on the user and walking speed and a typical waveform can be seen with the zoomed in Figure 2-2. The frequency can vary from 0 to $350 \mathrm{~Hz}$ with $320 \mathrm{~Hz}$ being the maximum frequency shown in both Figure 2-1 and Figure 2-2. 


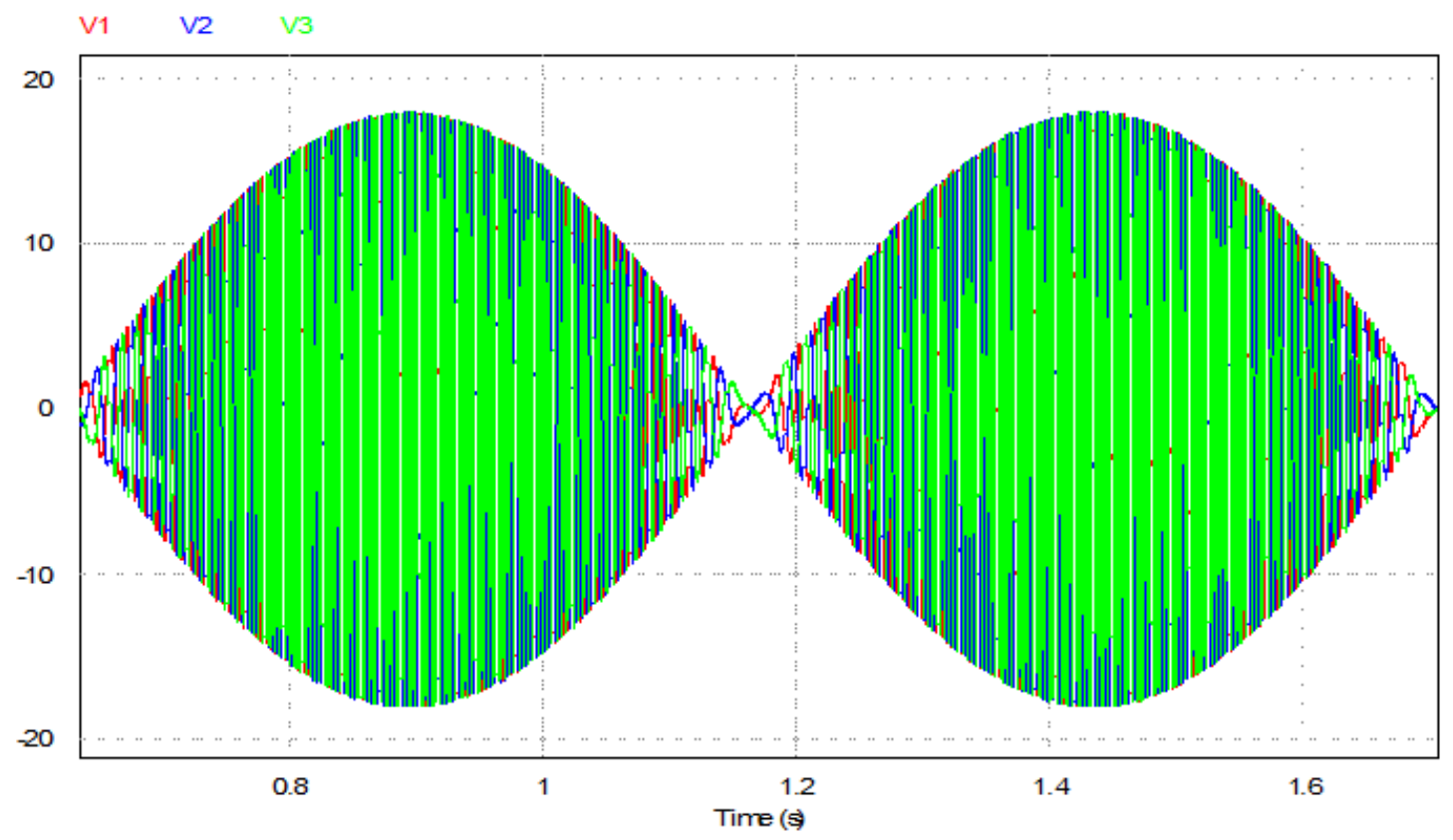

Figure 2-1 - Typical Three-Phase AC waveform from Human Energy Harvester with user walking at $1.5 \mathrm{~m} / \mathrm{s}(5.4 \mathrm{~km} / \mathrm{h})$

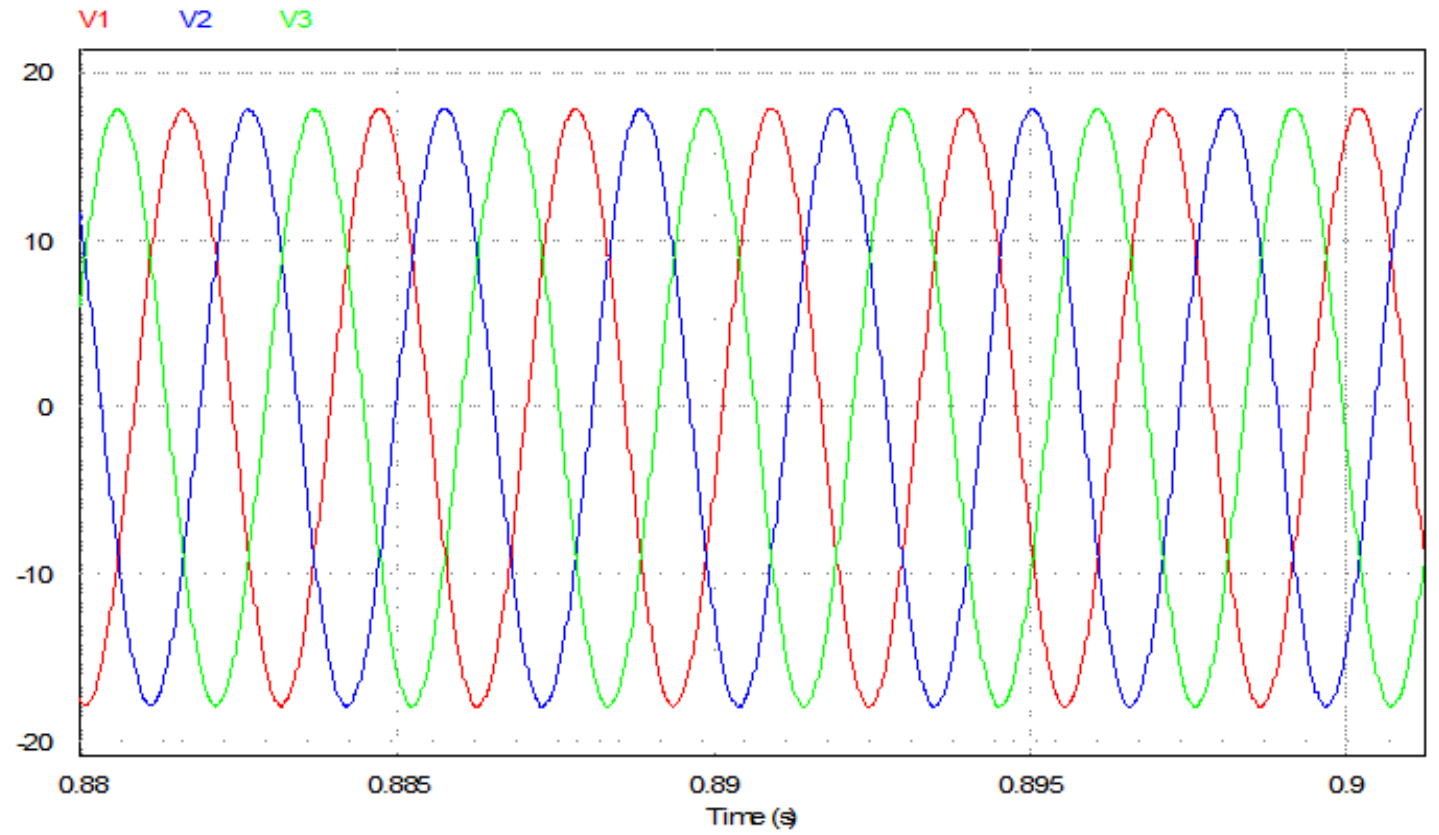

Figure 2-2 - Three-Phase AC waveform from Human Energy Harvester with user walking at $1.5 \mathrm{~m} / \mathrm{s}$ (Zoomed In) 
The peak voltage generated by the energy harvester is $35 \mathrm{~V}_{1-1}$ with the peak power around 53 watts and the average power around 11.5 watts with the user walking at a speed of $1.5 \mathrm{~m} / \mathrm{s}$.

It must be reiterated that the power source for this PEM, the human energy harvester, is very different from the conventional utility grid. With the human energy harvester the power that is available to be extracted is limited in comparison with the utility grid as well as the power is time varying at low frequency. All of the power that is generated potentially comes at the expense of high metabolic energy input from the user. In order for the design of the PEM to be effective it is vital for the PEM to extract power when the power is available and to store all of the power that is extracted. These limitations of the human energy harvester as an input power source will weigh in heavily in regards to the design considerations of the PEM. The different sections of the PEM will be described in the following sections starting with the choice of battery chemistry.

\subsection{Energy Storage Medium}

We will start the design of the PEM with the choice of battery chemistry used for storing the power extracted from the human energy harvester as the battery chemistry and battery size may impose some more design requirements on the PEM. As the PEM is being designed to be integrated into a backpack that will be carried by a human; size, weight and therefore energy density will be the deciding factor when choosing the battery chemistry. The three main categories of rechargeable batteries available are lead based, nickel based and lithium based batteries. Out of these three categories lithium based batteries have the highest energy densities as can be observed in Figure 2-3 comparing energy density and energy volume[19]. Based on the energy density as well as form 
factor, the Lithium Polymer battery will complement the system well and is therefore selected to be used as the battery choice for storage of energy in the PEM.

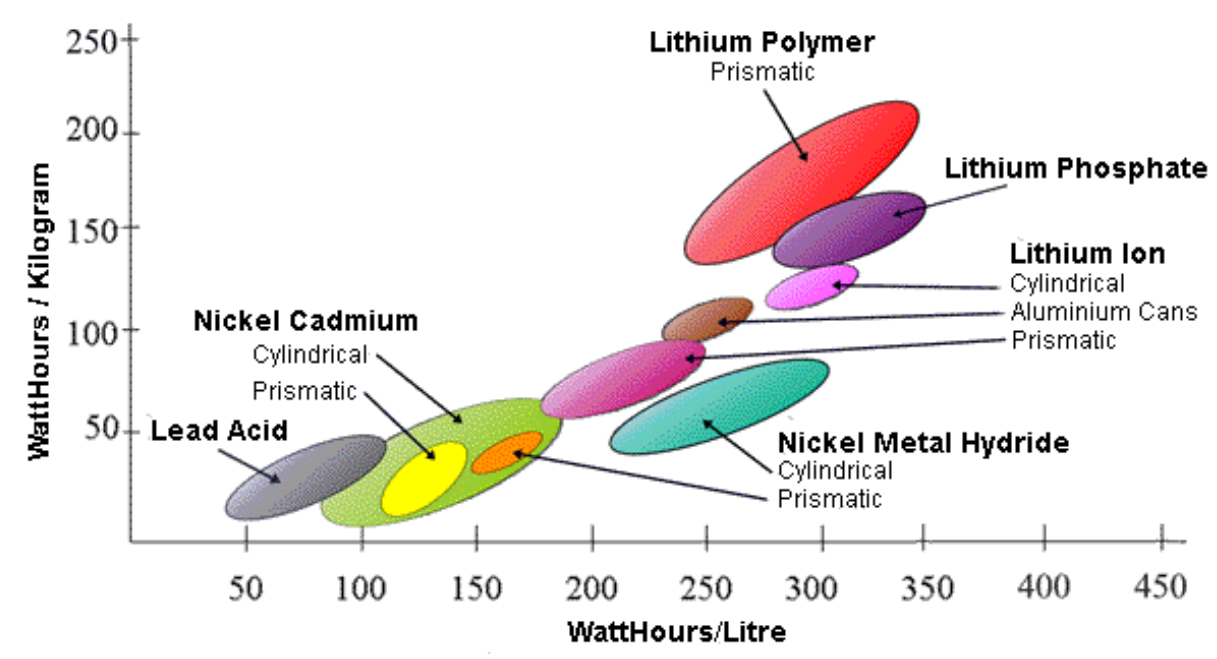

Figure 2-3 - Energy Densities of Secondary Battery Chemistries [20]

One of the disadvantages of the lithium polymer batteries is that they are very sensitive to overcharging. In order to maintain the safety of the battery the cell voltage must not exceed 4.2 Volts per battery cell. In addition to this, to prolong the lifetime of the battery as well as prevent the battery from rupturing, the charge current must not exceed the recommended charging current, usually $1 \mathrm{C}$ where $\mathrm{C}$ is the capacity of the battery [17]. As an example, a $2000 \mathrm{mAh}$ battery would have a $1 \mathrm{C}$ charge rate of $2 \mathrm{~A}$. The battery size selected should be large enough that it can sink the average power produced by the generator during the Constant Current (CC) or bulk charging cycle of the battery which will be equal to the $1 \mathrm{C}$ charge rate. The battery will be designed with the smallest capacity that is necessary to meet this requirement in order to minimize the cost, size and weight of the PEM. The use of the control algorithms developed, and design of the PEM will allow us to size the battery based on the average output power of the generator and not the peak power generated as was the case in other designs $[4,14]$. The smaller capacity battery will also be able to 
be fully charged for a shorter amount of walking time. The capacity of the battery can always be increased as more energy storage is needed without affecting the design of the converter or modifying the control.

As the lithium polymer batteries have limitations on the maximum charging current and voltage they can be charged at, the charging power of the battery is also limited. We must select the battery capacity based on the worst case scenario during the CC charging cycle of the battery. We know that during the $\mathrm{CC}$ charging cycle of the battery the charging current is limited to the $1 \mathrm{C}$ rate and the charging profile of the battery is shown in Figure 2-4. During this CC cycle we can also observe from the figure that the voltage increases from 2.8 volts per cell to 4.2 volts per cell after which the CC charging cycle of the battery is complete and the constant voltage (CV) phase of charging will start. From this charging current and voltage profile we can determine that the maximum charging power of the battery is lowest at the start of the CC charge cycle when the battery voltage is 2.8 volts per cell. As the batteries are not ideal, there are some additional losses due to the internal resistance of the battery. We can take this additional loss into account when determining the minimum of the maximum battery charging power, $\mathrm{P}_{\text {battmin. }}$ This will be taken as the worst case scenario when determining the capacity of battery to use. 


\section{Lithium Polymer}

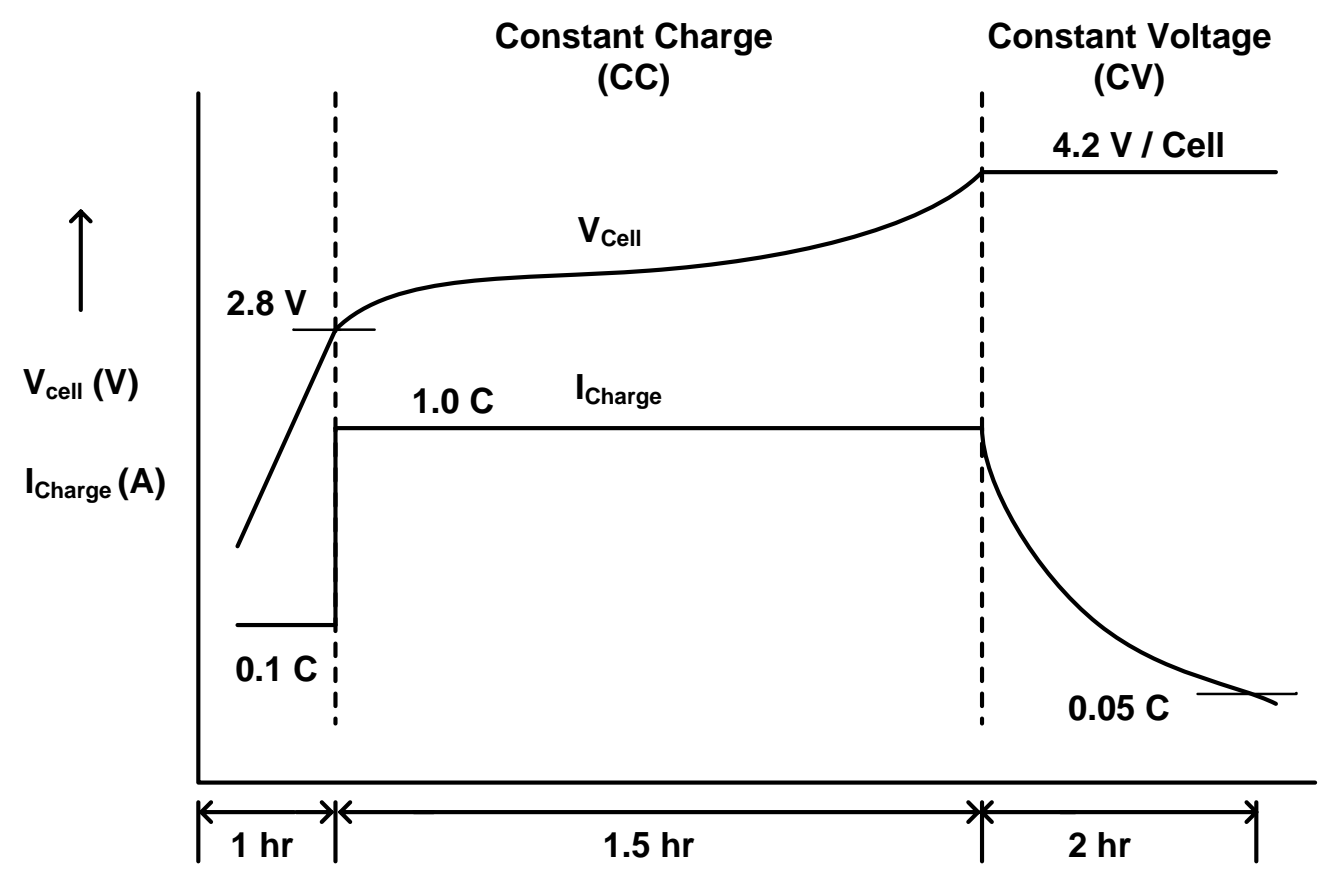

Figure 2-4 - Charging profile of Lithium Batteries [21]

$\mathrm{P}_{\text {battmin }}$ can be calculated using the equation (2.1), where $\mathrm{N}_{\text {Cells }}$ is the number of cells in the battery pack, $V_{\text {min }}$ is the minimum cell voltage of the battery, $R_{\text {batt }}$ is the internal resistance of the battery cell and $\mathrm{I}_{\mathrm{CCMAX}}$ is the constant current charging rate of the pack.

$$
P_{\text {battmin }}=\mathrm{N}_{\text {Cells }} *\left[V_{\text {min }}+R_{\text {batt }} * I_{\text {CCMAX }}\right] * I_{C C M A X}
$$

The average power generated from the human energy harvester, $\mathrm{P}_{\text {gen-avg }}$, is expected to be around 11.5 watts. We can use this as well as some additional information about the battery cells to determine our pack capacity. We have selected a battery with a capacity of 2000 mAh supplied by Union Battery, datasheet [22], with a $\mathrm{V}_{\min }=2.8 \mathrm{~V}, \mathrm{R}_{\text {batt }}=0.2$ and $\mathrm{I}_{\mathrm{CCMAX}}=2 \mathrm{~A}$. To determine the number of cells needed for our worst case, we set $\mathrm{P}_{\text {battmin }}>\mathrm{P}_{\text {gen-avg }}$ and rearrange (2.1) to get (2.2) 


$$
\mathrm{N}_{\text {Cells }}>\frac{\mathrm{P}_{\text {gen-avg }}}{\left[V_{\min }+R_{\text {batt }}{ }^{* I}\right. \text { CCMAX }}{ }^{* I_{C C M A X}}
$$

The battery parameters and average power generated from the energy harvester can be used in equation (2.2) to calculate the number of cells needed to meet this minimum requirement, which is equal to two cells. The nominal voltage of this battery pack will be 7.4 volts with an energy storage capacity of $14.8 \mathrm{Wh}$. The range of the maximum charging power of the battery pack will be from 12.8 - 16.8 W depending on the State of Charge (SoC) of the battery. The average power that is generated from the energy harvester will be less than $\mathrm{P}_{\text {battmin, }}$, however, the peak power of the generator will still need to be addressed. The peak power of the human energy harvester can generate close to 53 watts. Without a temporary means of energy storage the battery pack would need to have five times the capacity in order for it to safely store this peak power that is generated. As mentioned previously, this extra battery capacity is undesirable as it would add to the system weight, size and cost. Due to the varying input power generated by the human energy harvester, the peak power only lasts for a small portion of the power cycle, shown by the green waveform in Figure 2-5. As it is desirable to keep the battery pack small, the PEM must be able to store the excess energy temporarily when the input power generated, $\mathrm{P}_{\text {in }}$ is greater than the output power to the battery, $\mathrm{P}_{\text {out }}$ and later release that energy into the batteries once $\mathrm{P}_{\text {in }}$ has decreased below $\mathrm{P}_{\text {out }}$ as illustrated in Figure 2-5. The curves in Figure 2-5 are generated based on the expected power level of the human energy harvester generator and the output power level of the battery. 


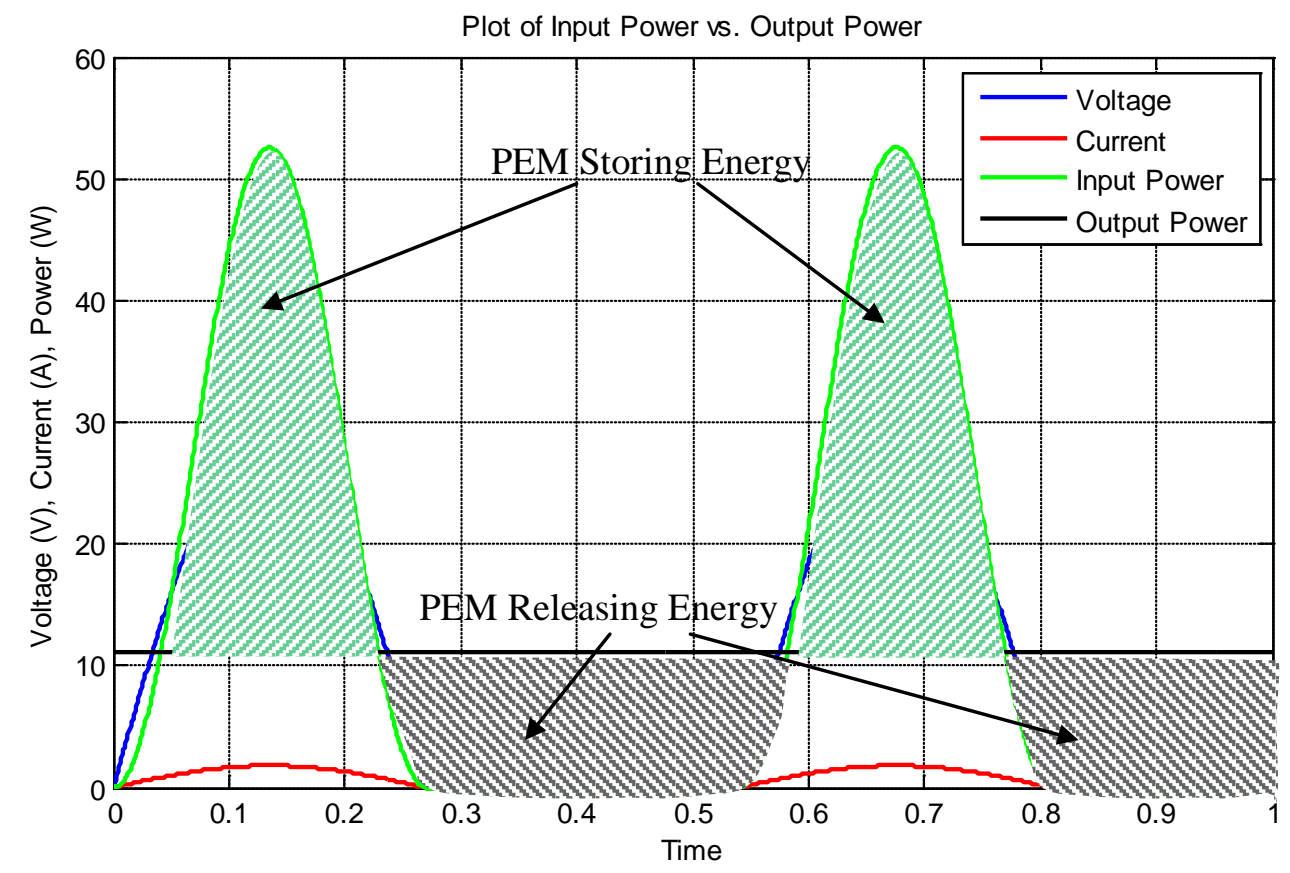

Figure 2-5 - Plot of Time Varying Input Power Generated by the Human Energy Harvester versus the Output Power to the Battery Pack

The PEM will store the extra energy harvested in a large bulk capacitor or an Energy Storage Capacitor (ESC) which will be discussed in Section 2.6.

\section{4 $\mathbf{R}_{\text {in }}$ Controlled Boost Converter}

In order to meet the design requirements needed to control the input power of the generator and charging rate of the lithium polymer batteries, the PEM has been designed as a two-stage power converter with an Energy Storage Capacitor (ESC) between the two stages, shown in Figure 2-6. The PEM also uses a three-phase full wave Schottky diode rectifier to rectify the input voltage. 


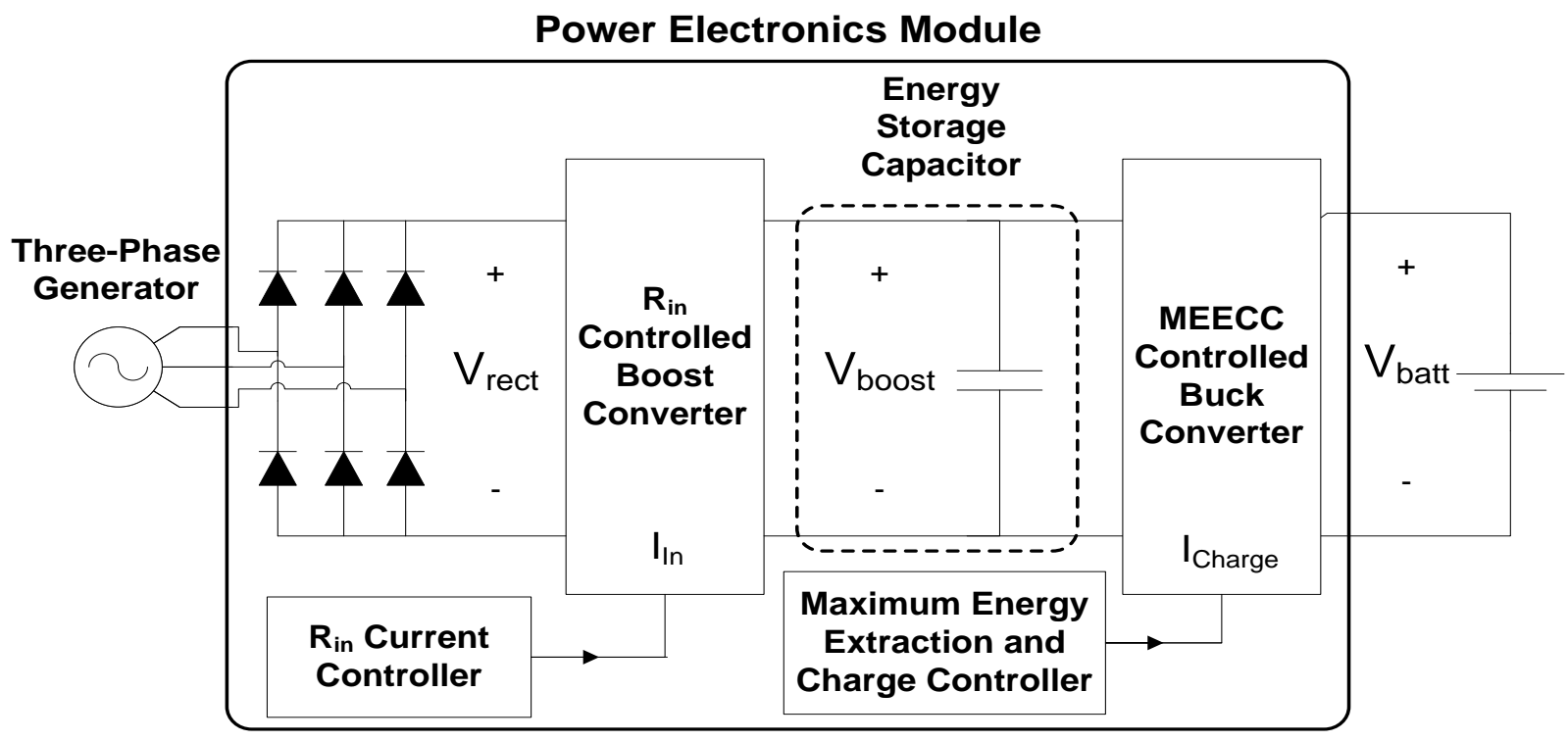

Figure 2-6 - Two-Stage Power Electronics Module with Energy Storage Capacitor

A Boost converter is used as the first stage of the two-stage power converter after the diode rectifier. The Boost converter stage is similar to that of a power factor correction (PFC) Boost converter with main differences [23] that will be outlined in Table 2-1. As detailed in Section 2.2, the output of the human energy harvester generator is a variable high-frequency three phase AC sinusoidal superimposed on a 1-2 $\mathrm{Hz}$ AC waveform. The three-phase generator is connected to the Schottky rectifier, shown in Figure 2-6, which creates the voltage $V_{\text {rect, }}$, shown in Figure 2-7 and the zoomed in view, Figure 2-8. The output of the rectifier is connected to the input of the $\mathrm{R}_{\text {in }}$ Controlled Boost Converter. 


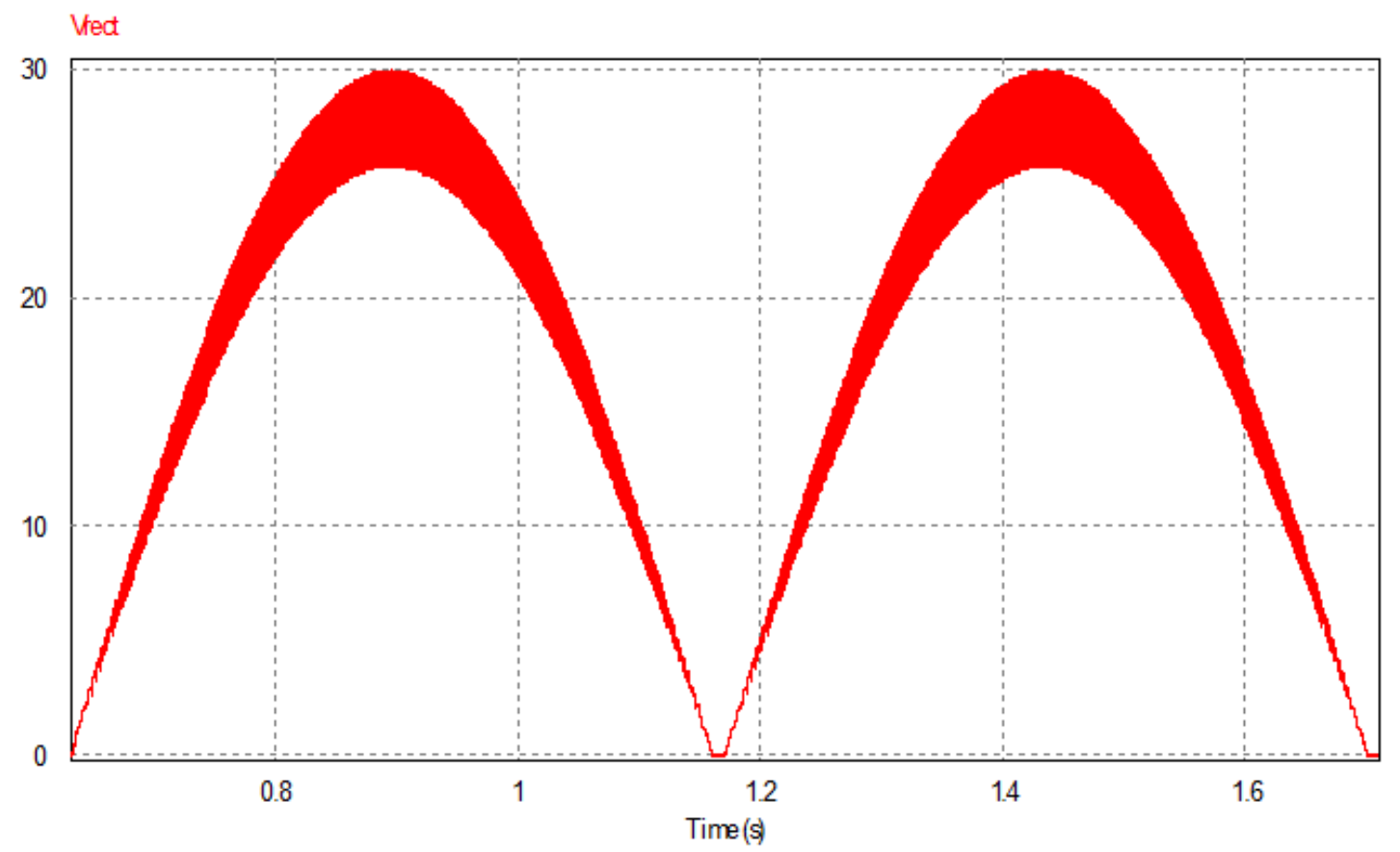

Figure 2-7 - Rectified Voltage from Generator Low Frequency AC envelope

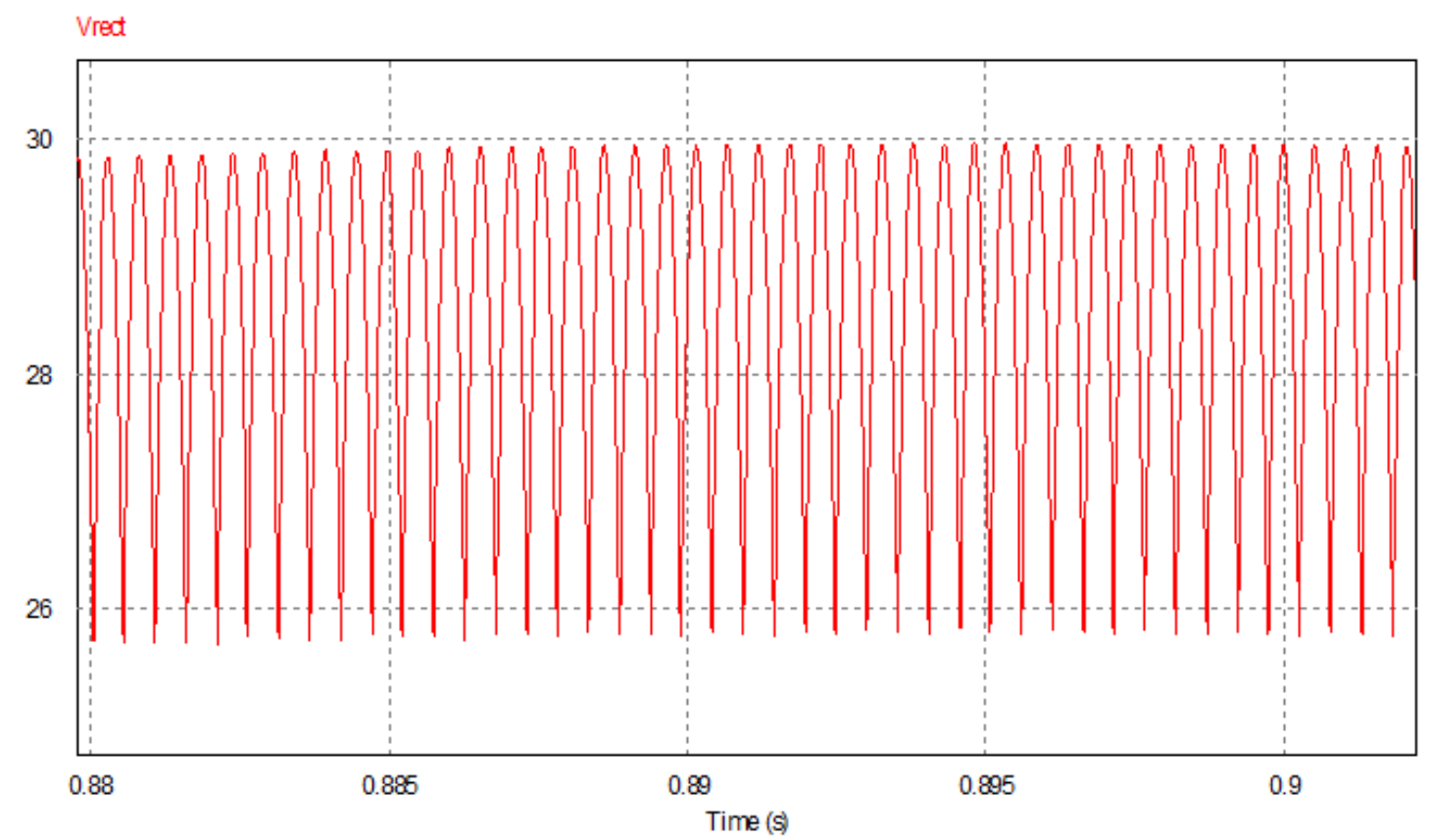

Figure 2-8 - Rectified Voltage from Generator High frequency Super Imposed Waveform (Zoomed in view of Figure 2-7) 
Similar to a Power Factor Controlled (PFC) Boost Converter, the $\mathrm{R}_{\text {in }}$ Controlled Boost Converter will emulate a resistive load for the generator with the advantage of the input current being in phase with the voltage resulting in power draw from the generator with a high power factor. The difference with the $\mathrm{R}_{\text {in }}$ Controlled Boost Converter is that the emulated resistor seen by the generator is the control variable. In the PFC Boost converter the resistor value that is emulated is changed by the voltage loop to regulate the output voltage of the Boost. The $\mathrm{R}_{\text {in }}$ Controlled Boost converter has no external voltage loop to set $\mathrm{R}_{\mathrm{in}}$, rather the value for the emulated resistor is set by the user directly to control how much power is extracted from the generator and how much user effort is required to turn the generator.

Table 2-1 - Comparison between Conventional PFC and proposed $\mathbf{R}_{\text {in }}$ Controlled Boost Converter

\begin{tabular}{|l|l|l|}
\hline & Conventional PFC & $\mathrm{R}_{\text {in }}$ Controlled Boost Converter \\
\hline Control Parameter & $\mathrm{V}_{\text {out }}$ & $\mathrm{R}_{\text {in }}$ \\
\hline Output Voltage & Regulated & Unregulated \\
\hline Input Frequency & $60 \mathrm{~Hz}$ & $1-2 \mathrm{~Hz}$ \\
\hline Seen by the Source as & Emulated Resistor controlled by & Emulated Resistor defined by \\
& $\mathrm{V}_{\text {out }}$ & user \\
\hline
\end{tabular}

As the $\mathrm{R}_{\text {in }}$ Controlled Boost converter does not have an outer voltage loop the Boost converter will no longer be responsible for regulating the output voltage $\mathrm{V}_{\text {boost }}$. The $\mathrm{R}_{\mathrm{in}}$ Controlled Boost converter will only be responsible for extracting current from the generator $\mathrm{I}_{\mathrm{in}}$ based on the relationship shown in (2.3)

$$
I_{\text {in }}=\frac{V_{\text {rect }}}{R_{\text {in }}}
$$


The $\mathrm{R}_{\text {in }}$ Controlled Boost converter will be extracting power from the generator according to the user's desired effort level by changing $\mathrm{R}_{\mathrm{in}}$. There is also an over voltage protection for the output of the $\mathrm{R}_{\text {in }}$ Controlled Boost converter in which the $\mathrm{R}_{\text {in }}$ value is increased if the voltage of the ESC reaches its maximum value in order to protect the components. The $\mathrm{R}_{\mathrm{in}}$ Controlled Boost converter will satisfy the design requirement of being able to draw current from the generator into any specified profile as determined by the user.

The Boost converter will also enable the PEM to draw current over the entire input voltage waveform allowing the extraction of power when the input voltage is lower than the output voltage. The $\mathrm{R}_{\mathrm{in}}$ value can also be changed dynamically so that power is extracted from the generator only during key generation points in order to optimize the ratio of user energy input to power extracted from the generator. As the Boost converter can no longer regulate the output voltage, this task will be passed on to the second stage of the PEM, the Maximum Energy Extraction and Charge Controlled Buck converter.

\subsection{Maximum Energy Extraction and Charge Controlled Buck Converter}

The second stage of the PEM is the Maximum Energy Extraction and Charge Controlled Buck converter, which will help the PEM meet two of the design requirements. The Buck converter is necessary to step down the voltage of the ESC to the battery charging voltage. The Buck converter will be responsible for minimizing the energy stored in the ESC by regulating the charging current of the lithium polymer battery pack to the CC limit in order to minimize the voltage stored in the ESC. The minimization of the energy stored in the capacitor is necessary to allow the $\mathrm{R}_{\mathrm{in}}$ Controlled Boost converter the most amount of capacity to store the extracted energy from the generator when $\mathrm{P}_{\text {in }}$ is greater than $\mathrm{P}_{\text {out }}$. If the ESC voltage, $\mathrm{V}_{\text {Boost }}$, reaches the maximum allowable 
voltage, $V_{\text {Boostmax }}$, while $P_{\text {in }}$ is greater than $P_{\text {out }}$ then we will have to reduce $P_{\text {in }}$ so that we do not damage the components. This will cause a reduction in the amount of energy extracted from the generator by the PEM. The energy stored in the ESC is proportional to the voltage of the capacitor squared, shown in (2.4)

$$
E=\frac{1}{2} C V^{2}
$$

The Buck converter will minimize this energy by regulating the voltage of the capacitor to its

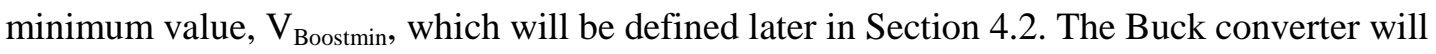
regulate the voltage of the ESC by controlling the charging current of the battery as long as the required current to regulate the voltage is within the safe charging limits of the battery. As long as $P_{\text {in }}$ is less than $P_{\text {out }}$ the Buck will be able to regulate the Boost voltage and minimal energy will be stored in the ESC. Once $\mathrm{P}_{\text {in }}$ becomes higher then $\mathrm{P}_{\text {out }}$, the extra energy will need to be temporary stored and the voltage of the ESC will no longer be regulated by the Buck converter and start to increase. The voltage of the ESC will continue to rise as long as $\mathrm{P}_{\text {in }}$ is greater than $\mathrm{P}_{\text {out }}$ until it reaches the over voltage protection level, in which case the input power is reduced by the Boost converter in order to protect the components. As $\mathrm{P}_{\text {out }}$ is based on the charging current and charging voltage to the battery, $\mathrm{P}_{\text {out }}$ will vary with the State of Charge (SoC) of the battery as discussed in Section 2.3.

The MEECC Controlled Buck converter will control the charging current and voltage of the battery pack to maximize safety of the batteries while regulating the voltage of the ESC to its minimum value maximizing the amount of power transfer from the ESC to the battery pack. 


\subsection{Energy Storage Capacitor}

The need for intermediate energy storage has been illustrated in the previous sections in order to reduce the required size, weight and cost of a battery pack that would be otherwise needed to sink the instantaneous power generated without intermediate energy storage. An Energy Storage Capacitor would provide a much smaller and lighter solution to the large battery pack without sacrificing potential energy harvest. The ESC will also help to charge the battery pack faster due to the larger energy harvest and smaller battery pack size. The voltage of the capacitor needs to be rated to a voltage greater than the input voltage with the voltage and size of the capacitor to be chosen based on the required amount of energy to be stored. A design guide will be provided in Section 5.3 to aid in selecting the required voltage and size of capacitor needed for a given input power level.

Ultra-capacitors or Electrochemical Double Layer Capacitors (EDLC) were also considered in the design, however, due to the voltage rating that is required of the capacitor as well as the reduced lifetime of the EDLC, it is not feasible to use these types of capacitors. The capacitor that was chosen for this design was the aluminum electrolytic capacitor due to the voltage rating and required capacitance. The ESC allows the PEM to store the instantaneous power that would otherwise not be able to be harvested from the generator, allowing the battery pack to charge quicker. The capacitor also has the advantage that the batteries receive a fully saturated $1 \mathrm{C}$ charge rate that is closer to a pure DC charging current.

\subsection{Conclusion}

This chapter proposed the system block diagram for the Power Electronics Module that was designed to solve the problems associated with the previous designs, as well as provide some 
additional functions for a newly developed generator provided by the collaborating mechanical engineers. The PEM will draw current in any profile as desired by the user to control the amount of power extracted. The extracted power will be stored into lithium polymer batteries while adhering to the charging limitations of the reduced size battery pack. The PEM design consists of two stages, a $\mathrm{R}_{\mathrm{in}}$ Controlled Boost converter followed by a Maximum Energy Extraction and Charge Controlled Buck converter. An Energy Storage Capacitor is placed between the two stages allowing the temporary storage of energy when the input power is greater than the charging power of the battery, allowing the battery pack size to be reduced while not limiting the amount of energy extracted. The design of the two stages will allow the PEM to meet the design requirements of extracting the maximum amount of power from the generator as desired by the user and safely storing the extracted energy into an appropriately sized lithium polymer battery pack while within the charging limitations of the battery. 


\section{Chapter 3}

\section{Control Strategy of $\mathbf{R}_{\text {in }}$ Controlled Boost Converter}

\subsection{Introduction}

This chapter presents the detailed implementation and analysis of the control methods used to control the $\mathrm{R}_{\mathrm{in}}$ Controlled Boost converter and how they help the PEM achieve the design requirements outlined in Chapter 2. The design requirements of the Boost converter are that it must be able to extract the maximum power as defined by the user that is available from the limited power that is generated by the generator. The Boost converter must also be able to draw current from the generator in any desired waveform profile or shape that is defined by the user. In order to meet these design requirement there are three different control schemes that control the input current to the Boost converter depending on the desired mode of operation.

The first mode of operation is the Constant $R_{\text {in }}$ Control mode. This mode of control allows the user to select the resistor value for the Boost converter to emulate and will draw current that is proportional to the input voltage. The second mode of control is the Variable $\mathrm{R}_{\mathrm{in}}$ Control mode. This method is similar to the Constant $\mathrm{R}_{\mathrm{in}}$ Control mode but allows the user to specify different resistor values to emulate depending on the input voltage. The third mode of control is the Dynamic $\mathrm{R}_{\mathrm{in}}$ Control mode. This mode of control uses an electrical signal from the mechanical generator or a computer for the resistor value to emulate. This mode of control will be used by the bio-mechanical engineers to fine tune the energy extraction profile to the specific user's gait cycle in an attempt to minimize the metabolic cost of the generation of the energy. This chapter will also cover the derivation of the digital control loop, the requirements of the Boost compensator and the prototype that was designed. Experimental results are presented to show the effectiveness of the 
control system and Boost converter in achieving the design requirements of the PEM. The next section in this chapter will cover the design of the Constant $\mathrm{R}_{\mathrm{in}}$ Controller.

\subsection{Constant $\mathbf{R}_{\text {in }}$ Control}

The first mode of control of the Boost converter is the Constant $R_{\text {in }}$ Control mode. This mode of control allows the user to select the value of the resistor for the Boost converter to emulate for the generator. The Constant $\mathrm{R}_{\mathrm{in}}$ Control allows the PEM to extract more power from the generator than a conventional PFC controller because the Constant $\mathrm{R}_{\text {in }}$ Control emulates a constant resistor value based on the user's desired value as opposed to the conventional PFC controller which emulates the input resistor based on the output power requirements of the Boost converter.

The output power of a conventional PFC controller is determined by the load it is supplying and will vary the input power to match the output power. The output power is controlled by controlling the $\mathrm{R}_{\mathrm{in}}$ value to regulate the output voltage level of the Boost converter. The relationship between input power, $\mathrm{P}_{\text {in }}$, output power, $\mathrm{P}_{\mathrm{o}}$, input voltage, $\mathrm{V}_{\text {in }}$ and the emulated resistor, $\mathrm{R}_{\text {in }}$ is given in equation (3.1).

$$
P_{\text {in }}=P_{\text {out }}=\frac{V_{\text {in }}^{2}}{R_{\text {in }}}
$$

Equation (3.1) can be rearranged to find the value for the emulated input resistor $\mathrm{R}_{\mathrm{in}}$ that is required to supply the output power level and is shown in equation (3.2)

$$
R_{\text {in }}=\frac{V_{\text {in }}^{2}}{P_{\text {out }}}
$$

As the conventional PFC controller's emulated input resistor value is based on the output power requirements of the converter and not based on extracting all of the limited power that is available from the generator, the conventional PFC controller is not the optimal controller for maximizing the 
amount of energy harvest from the generator. The Constant $\mathrm{R}_{\mathrm{in}}$ Controlled Boost converter is better suited to control the amount of input power drawn from the generator as the emulated input resistor is based on the user's desired level and not based on regulating the output voltage of the Boost converter.

Without the need to regulate the output voltage, the Constant $\mathrm{R}_{\text {in }}$ Controlled Boost converter can keep the emulated input resistance constant. This will allow the Constant $\mathrm{R}_{\text {in }}$ Controlled Boost converter to extract power from the generator according to the relationship shown in (3.3) where $\mathrm{V}_{\text {rect }}$ is the rectified input voltage and $\mathrm{R}_{\mathrm{in}}$ is the user's desired input resistance value.

$$
P_{\text {in }}=\frac{V_{\text {rect }}{ }^{2}}{R_{\text {in }}}
$$

The input current waveform will be proportional to the rectified input voltage and inversely proportional to the $\mathrm{R}_{\mathrm{in}}$ value, shown in equation (3.4)

$$
I_{\text {in }}=\frac{V_{\text {rect }}}{R_{\text {in }}}
$$

From equation (3.4), we can observe that the input current waveform will be the same shape as the input voltage. This will create constant and predictable loading of the generator. This is a key feature of the Constant $\mathrm{R}_{\text {in }}$ Control, as the user can feel the loading of the generator. If the loading were too great or unpredictable it could cause the user to trip. The resistance felt by the user will be proportional to both the selected $\mathrm{R}_{\mathrm{in}}$ value and the speed they are walking. The resistor setting is analogous to the difficulty level used in some exercise equipment where the amount of work required can be changed by changing the difficulty setting.

The Constant $\mathrm{R}_{\text {in }}$ Controller also has the benefit of the input voltage and input current being in phase with each other with a power factor close to unity. This helps to extract more power from the 
generator as it increases the ratio of real power to apparent power, reducing the circulating losses in the generator. The key to extracting the maximum available power from the generator lies in the ability for the user to choose the emulated $\mathrm{R}_{\text {in }}$ value of the Boost converter.

The Constant $\mathrm{R}_{\mathrm{in}}$ Controller is realized in the digital domain by dividing the sampled input voltage $V_{\text {rect }}$ by the user's desired input resistor value, $R_{\text {in }}$ with the resultant creating the current reference for the Boost Average Current Controller's two-pole two-zero Compensator, $\mathrm{I}_{\mathrm{in}}$, shown in Figure 3-1.

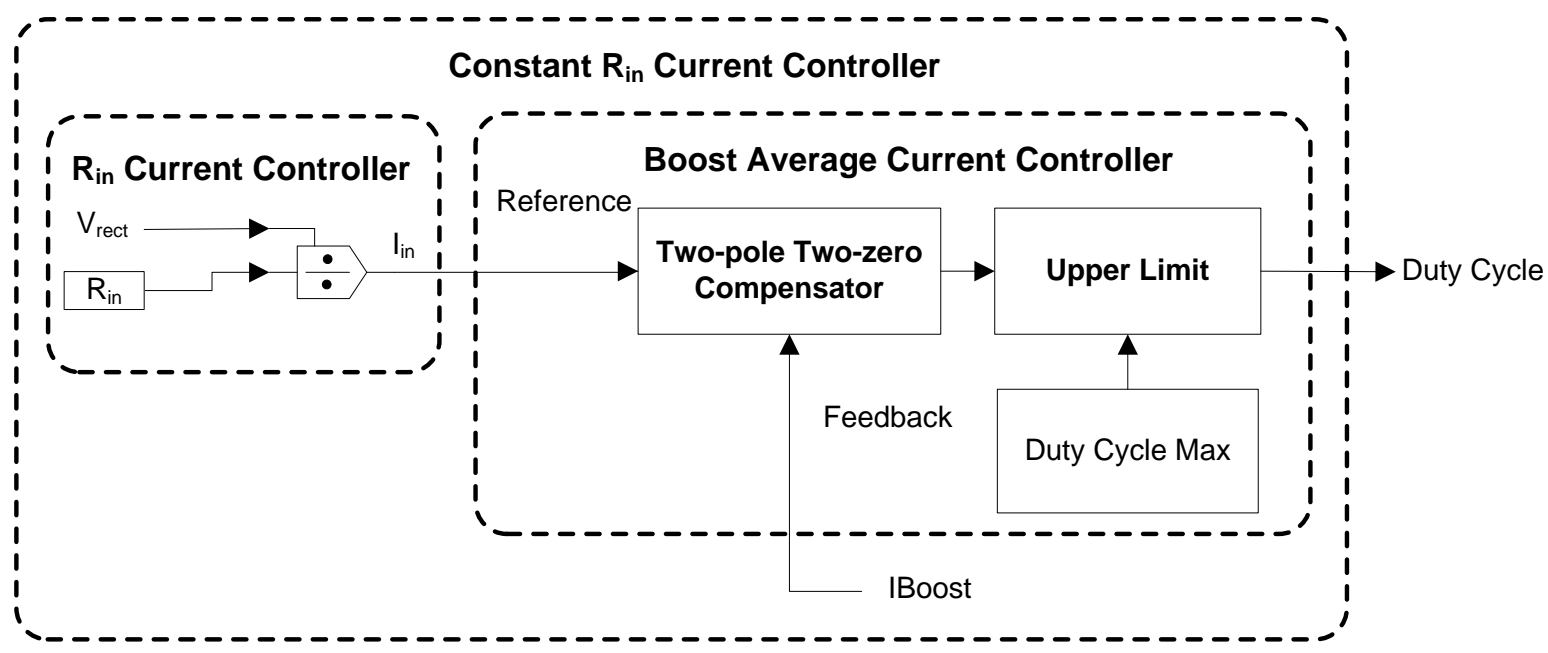

Figure 3-1 - Constant $\mathbf{R}_{\text {in }}$ Current Controller

The feedback signal for the two-pole two-zero compensator, $\mathrm{I}_{\mathrm{Boost}}$, is the ADC sampled value from the current sense amplifier (CSA). The CSA amplifies the differential voltage across the current sense resistor, $\mathrm{R}_{\text {sense, }}$ which is proportional to the Boost inductor current as can be observed in Figure 3-2. 


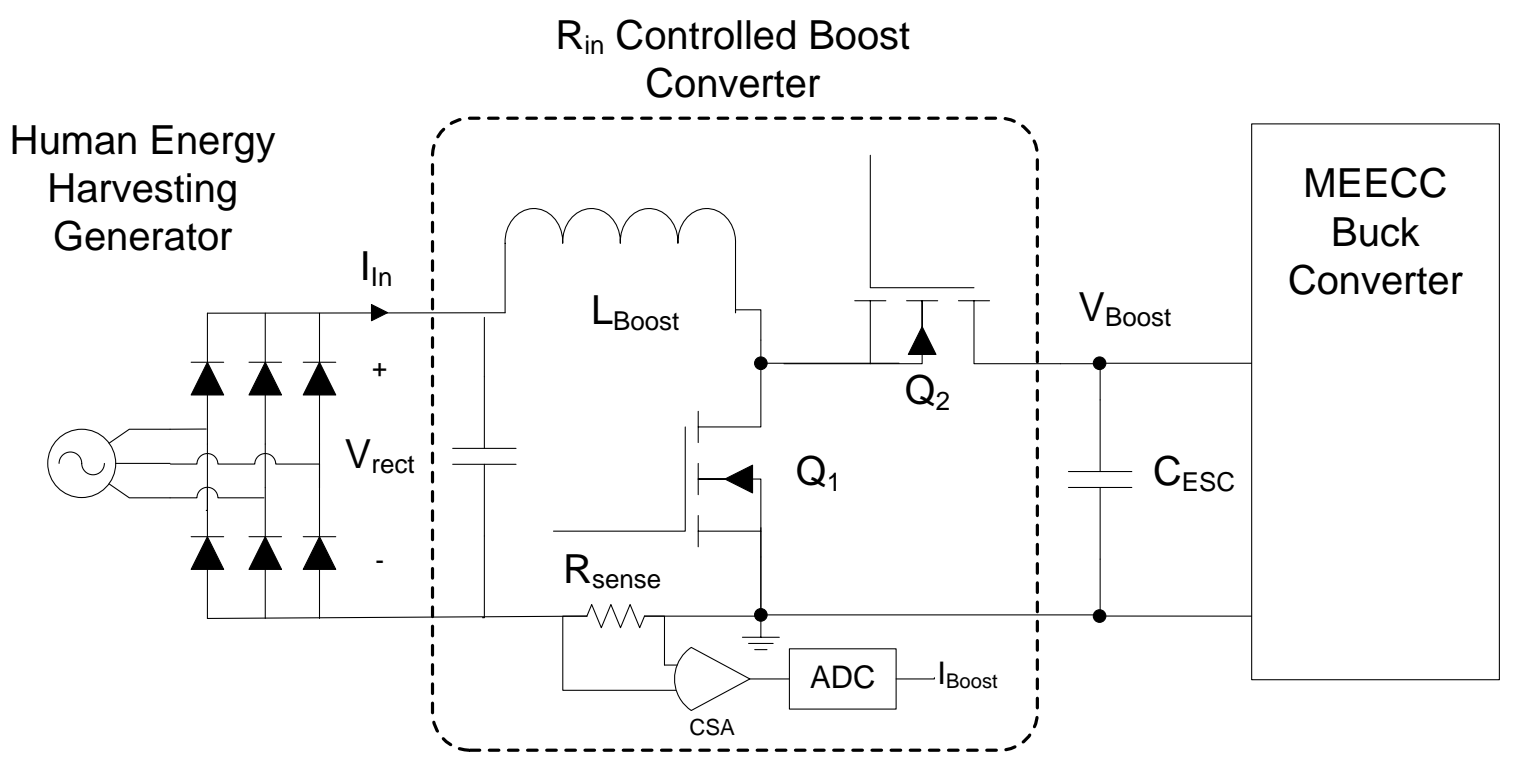

Figure 3-2 - Schematic of $\mathbf{R}_{\text {in }}$ Controlled Boost Converter

The average inductor current is also equal to the average input current, $\mathrm{I}_{\mathrm{in}}$, to the $\mathrm{R}_{\mathrm{in}}$ Controlled Boost Converter. The difference between the reference signal and feedback signal creates the error signal that is used by the two-pole two-zero compensator to vary the duty cycle of Q1 to control the average input current of the $\mathrm{R}_{\text {in }}$ Controlled Boost Converter. The details and design of the compensator will be covered in Section 3.5.

\subsection{Variable $\mathbf{R}_{\text {in }}$ Control}

The next mode of control of the $\mathrm{R}_{\text {in }}$ Controlled Boost Converter is the Variable $\mathrm{R}_{\text {in }}$ Control. This mode of control is similar to the Constant $R_{\text {in }}$ Control. However, the value of $R_{\text {in }}$ is changed based on the input voltage level in order to shape where the majority of the power is extracted during the user's gait cycle. This mode of control is useful as the voltage produced by the generator corresponds to different phases of the user's walking gait cycle, thus allowing the PEM to draw more power during specific points along the user's gait cycle based on the input voltage. By 
shaping where the majority of power is extracted, the amount of effort required to drive the generator can be fine-tuned to reduce the impact that generation has on the human walking.

The Variable $\mathrm{R}_{\text {in }}$ Controller will calculate the reference current, $\mathrm{I}_{\mathrm{in}}$, shown by equation (3.5).

$$
\begin{gathered}
I_{\text {in }}=\frac{V_{\text {rect }}}{R_{\text {in }}} \\
R_{\text {in }}=R_{1}, \text { when } V_{\text {rect }}<V_{1} \\
R_{\text {in }}=R_{2}, \text { when } V_{1}<V_{\text {rect }}<V_{2} \\
R_{\text {in }}=R_{3} \text {, when } V_{\text {rect }}>V_{2}
\end{gathered}
$$

The value of $R_{\text {in }}$ that is selected can be one of the three user preset values, $R_{1}, R_{2}, R_{3}$ depending on the value of the input voltage $V_{\text {rect }}$ compared to two threshold voltage levels, $V_{1}$ and $V_{2}$ which are also preset by the user. The first threshold level $V_{1}$ represents the level below which no current will be drawn by the Boost converter as the $\mathrm{R}_{1}$ value in this case is a large value to represent a large resistor. The value of the resistor $\mathrm{R}_{2}$ represents the resistor value when a reduced input power level is desired. This is the case when $V_{\text {rect }}$ is greater than the first voltage threshold level $V_{1}$ but less than the second voltage threshold level $V_{2}$. The third value of the resistor, $R_{3}$ is the full power case and is represented when $V_{\text {rect }}$ is greater than the second voltage threshold level $V_{2}$. In this case the Boost converter will be drawing full power.

Similar to the Constant $\mathrm{R}_{\mathrm{in}}$ Control, the reference current is passed on to the Boost Average Current Controller two-pole two-zero compensator to calculate the duty cycle for the Boost converter as shown in Figure 3-3. The compensator is the same as the Constant $\mathrm{R}_{\mathrm{in}}$ Control and the design details will be discussed in Section 3.5 


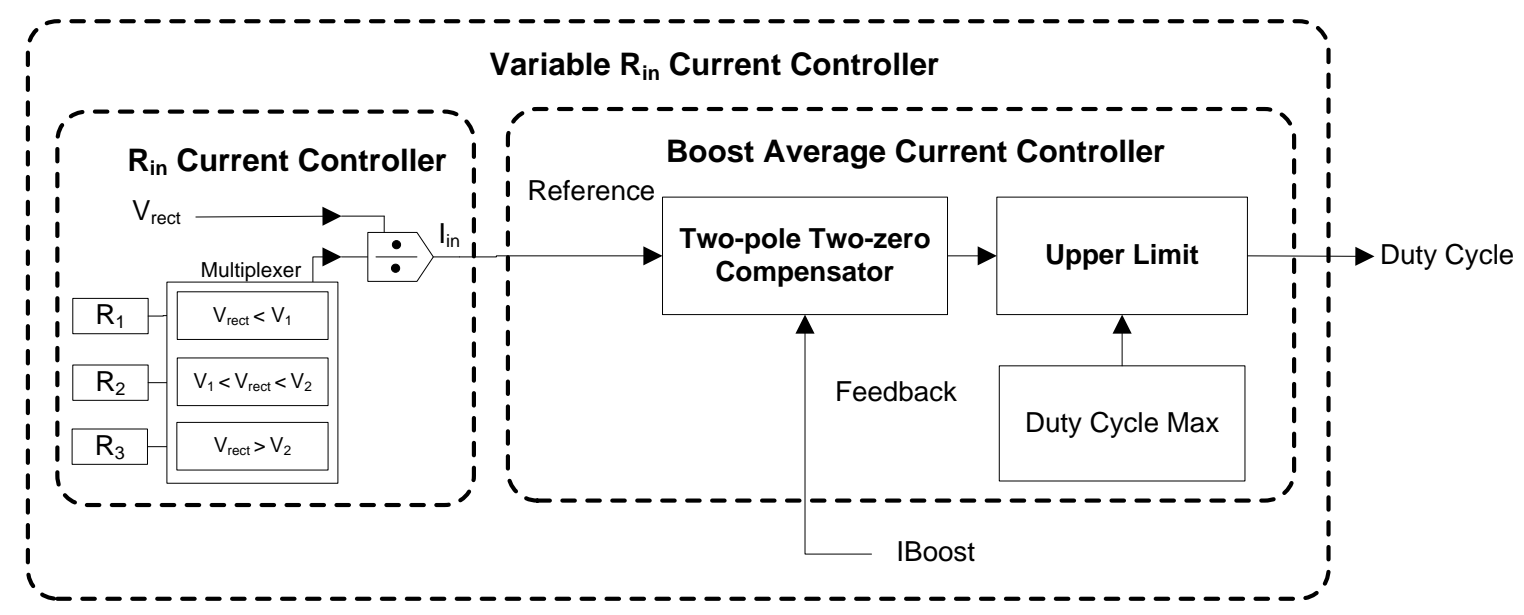

Figure 3-3 - Variable $\mathbf{R}_{\text {in }}$ Current Controller

The Variable $\mathrm{R}_{\text {in }}$ Control would extract more power from the generator when the input voltage, $\mathrm{V}_{\text {rect }}$ is higher than the threshold $V_{2}$ and less power when $V_{\text {rect }}$ is between the two threshold values $V_{1}$ and $\mathrm{V}_{2}$. The Variable $\mathrm{R}_{\mathrm{in}}$ Control can also disable the Boost converter when the input voltage is too low for conversion denoted by a $V_{\text {rect }}$ less than $V_{1}$. An example of the waveforms that would be expected from the Variable $\mathrm{R}_{\mathrm{in}}$ Controller can be observed in Figure 3-4. 


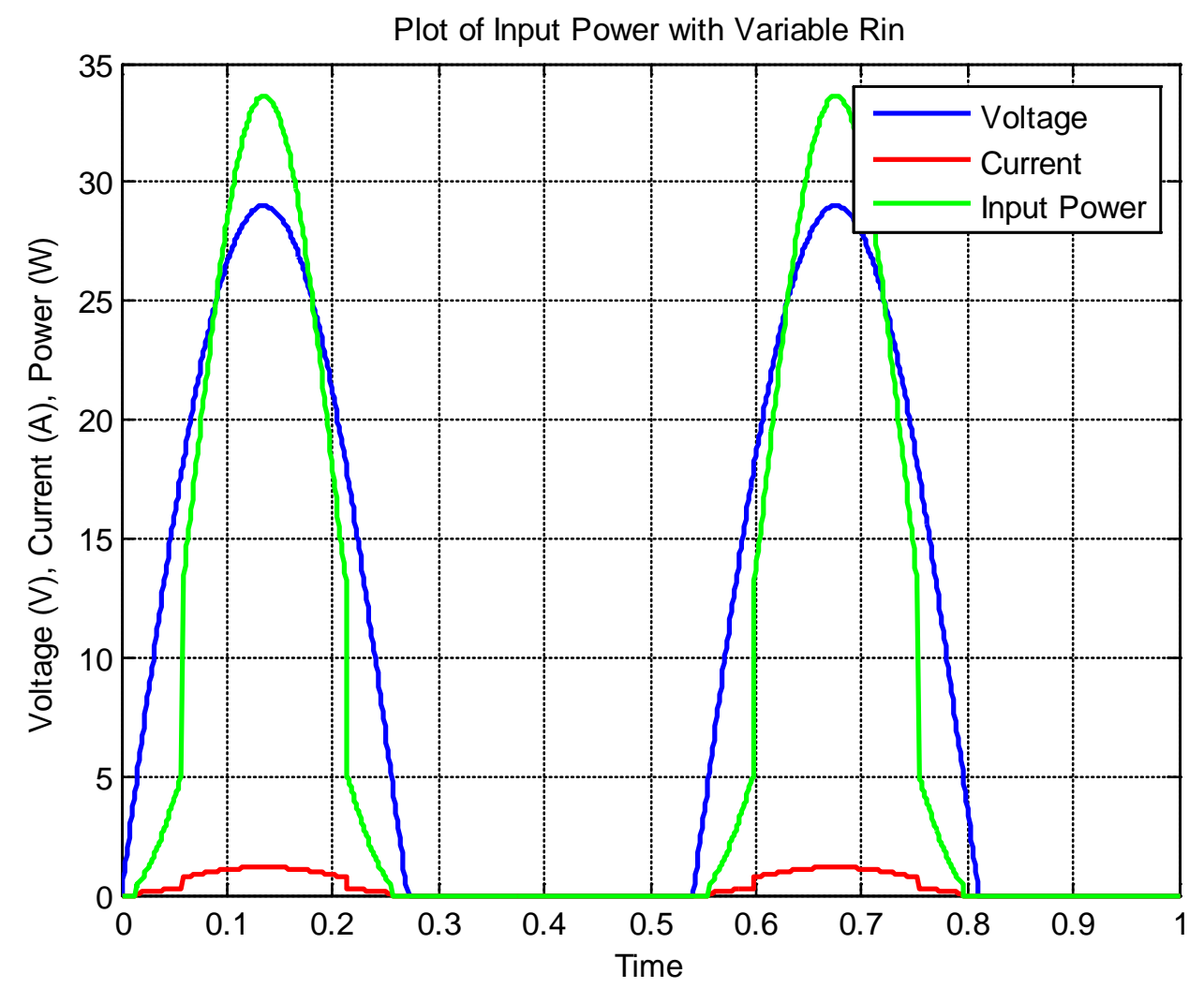

Figure 3-4 - Plot of Input Power with Variable $\mathbf{R}_{\text {in }}$ Controlled Boost Converter

In the example waveform, the $\mathrm{R}_{\text {in }}$ is set to be very large when $\mathrm{V}_{\text {rect }}<5 \mathrm{~V}, \mathrm{R}_{\text {in }}=50 \Omega$ when $5 \mathrm{~V}<$ $\mathrm{V}_{\text {rect }}<18 \mathrm{~V}$ and $\mathrm{R}_{\text {in }}=25 \Omega$ when $\mathrm{V}_{\text {rect }}>18 \mathrm{~V}$. It can be observed in the figure that input power waveform varies with both the input voltage and input resistor. The input power is higher when the input voltage is above $18 \mathrm{~V}$ and the input power is reduced when the input voltage is between $5 \mathrm{~V}$ and $18 \mathrm{~V}$. There is no power drawn when the input voltage is below $5 \mathrm{~V}$.

\subsection{Dynamic $\mathbf{R}_{\text {in }}$ Control}

The third mode of control for the $\mathrm{R}_{\text {in }}$ Controlled Boost converter is the Dynamic $\mathrm{R}_{\mathrm{in}}$ Control mode. This mode of control will control the input resistor value of the Boost converter directly based on a real-time voltage given to the PEM through a $\mathrm{R}_{\text {in }}$ control input sensed by the Analog to Digital Converter (ADC). This will allow current to be drawn from the generator in any shape or profile. 
This control mode is useful for when different amounts of power are needed to be drawn during key points of the user's gait cycle, similar to the Variable $\mathrm{R}_{\text {in }}$ Control mode. The difference with the Dynamic $\mathrm{R}_{\mathrm{in}}$ Control mode is that the resistor value is not dependent on input voltage and is not limited to only three resistor values. The value of the input resistor can be continuously changed as determined by the voltage on the $\mathrm{R}_{\mathrm{in}}$ control input. This allows the generation of energy to be further optimized to reduce the amount of user effort it requires to drive the generator while still extracting power. The Dynamic $\mathrm{R}_{\text {in }}$ Control will maximize the power extracted to energy input ratio as this mode of control imposes no limitations on the value of the input resistor emulated.

The Dynamic $\mathrm{R}_{\mathrm{in}}$ Current Controller creates the reference current signal, $\mathrm{I}_{\mathrm{in}}$, for the Boost Average Current Controllers two-pole two-zero compensator based on equation (3.4). The $\mathrm{R}_{\text {in }}$ value is the scaled sensed voltage value of the $\mathrm{R}_{\mathrm{in}}$ Control Input as shown in Figure 3-5. The two-pole two-zero compensator is the same as the two previous control modes and again will be discussed in more detail in Section 3.5

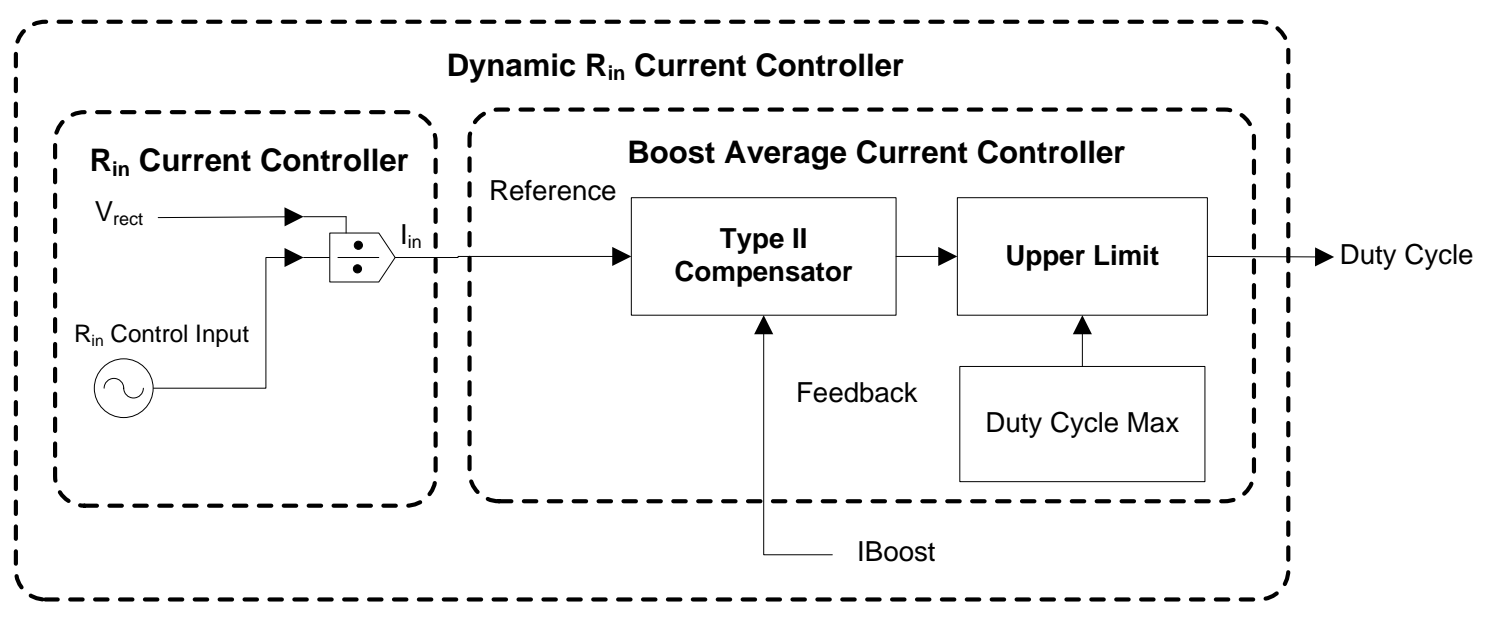

Figure 3-5 - Dynamic $\mathbf{R}_{\text {in }}$ Current Controller 
The voltage profile on the $\mathrm{R}_{\text {in }}$ control pin can be created using either a computer, or can be derived using mechanical feedback from sensors on the generator. The extraction of power can also be discontinuous, allowing the extraction of power for only a portion of the user's gait cycle, regardless of the input voltage. Power can be extracted during the latter half of the generation cycle harvesting the negative power produced by the knee as demonstrated in [11]. This can be used to generate power that is analogous to regenerative braking of a car which will help conserve human energy while still extracting power from the generator. An example of how the input power will vary with the Dynamic $R_{\text {in }}$ Control and sensed $R_{\text {in }}$ value is shown in

Figure 3-6.

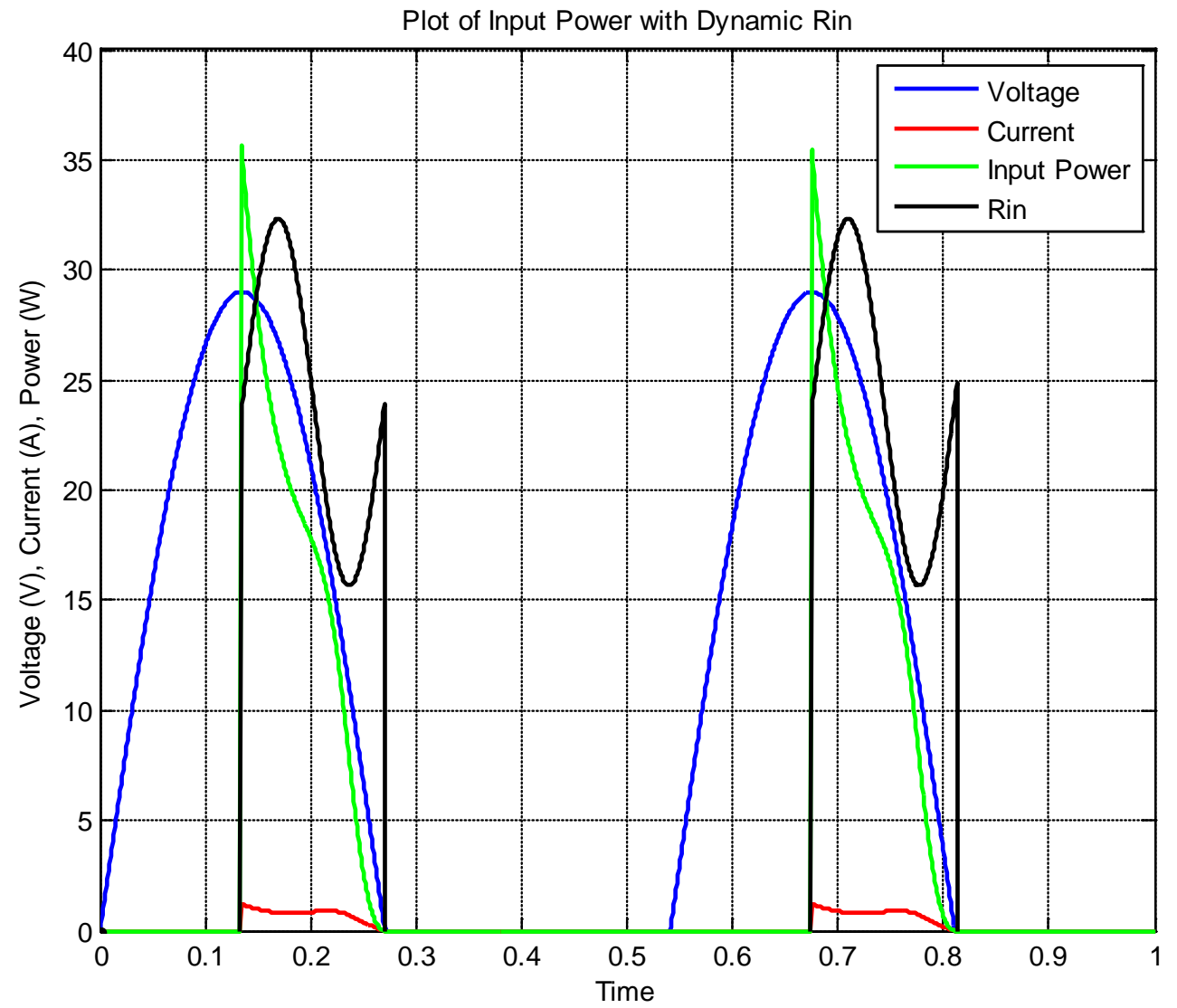

Figure 3-6 - Plot of Input Power with Dynamic $\mathbf{R}_{\text {in }}$ Controlled Boost Converter 
The voltage profile used for $\mathrm{R}_{\mathrm{in}}$ is a discontinuous waveform to show that the power draw can be discontinuous. The $\mathrm{R}_{\text {in }}$ value is very large for the first half of the input cycle and is shown as a null waveform. The second half of the cycle is a sinusoid to demonstrate that $\mathrm{R}_{\mathrm{in}}$ can be any arbitrary waveform. Both the input current profile and input power profile are shown in the figure. The figure shows that the input power profile is discontinuous, only drawing power during the second half of each input voltage cycle as determined by the sensed $R_{\text {in }}$ value. The profile of $R_{\text {in }}$ can be of any shape and a sinusoid was shown only for demonstration purposes.

\subsection{Prototype Requirements and Design of $\mathbf{R}_{\text {in }}$ Controlled Boost Converter}

In order to validate and fine tune the control theories a prototype digitally controlled average current mode control Boost converter was designed with the following design parameters: $\mathrm{V}_{\text {in }}=0$ -

$35 \mathrm{~V}$ peak, $\mathrm{F}_{\text {in }}=0-2 \mathrm{~Hz}, \mathrm{~V}_{\text {boost }}=6-80 \mathrm{~V}, \mathrm{f}_{\mathrm{sw}}=250 \mathrm{kHz}, \mathrm{L}_{\text {Boost }}=180 \mu \mathrm{H}, \mathrm{C}_{\mathrm{ESC}}=2200 \mu \mathrm{F}, \mathrm{R}_{\text {sense }}=0.02$ $\Omega, \mathrm{I}_{\mathrm{in}}=0-4 \mathrm{~A}, \mathrm{P}_{\text {inpeak }}=140 \mathrm{~W}, \mathrm{P}_{\mathrm{avg}}=15 \mathrm{~W}$. A TMS320F2808 digital signal processor (DSP) was used for the control of the Boost converter. The DSP that is used in the prototype is much more computational power then is required for the application and in the future can be scaled down to reduce cost and conserve energy. The controller is realized in the digital domain to aid in quicker tuning of the controller as well as other benefits of using digital control that will be mentioned later in this thesis. The components of the power stage of the Boost converter were designed for the converter to operate in continuous conduction mode (CCM) based on the Boost design equations given in [24]. The values determined from the Boost design equations were used to select the parts that were used in the Boost converter and are listed in Table 3-1. 
Table 3-1 - Table of Components used in Boost Converter

\begin{tabular}{|l|l|l|l|}
\hline Part & Part Number & Manufacturer & Value \\
\hline Control MOSFET & NTD6416ANT4G & ON Semiconductor & $100 \mathrm{~V}, 17 \mathrm{~A}, \mathrm{Rds} 81 \mathrm{~m} \Omega$ \\
\hline Synchronous MOSFET & IPD70N10S3-12 & Infineon Technologies & $100 \mathrm{~V}, 70 \mathrm{~A}, \mathrm{Rds} 11.1 \mathrm{~m} \Omega$ \\
\hline Inductor & PCV-2-184-05L & Coilcraft & $180 \mu \mathrm{H}, 4 \mathrm{Arms}$, Dcr $0.092 \Omega$ \\
\hline Driver & LTC4444IMS8E-5\#PBF & Linear Technology & $114 \mathrm{~V}$ Bootstrap, 1.4 Amp \\
\hline Sense Resistor & LVK12R010DER & Ohmite & R=0.020 $\Omega$ \\
\hline Op Amp & OPA2335AIDR & Texas Instruments & GBW 2 MHz \\
\hline Diode Rectifier & SSB44-E3/52T & Vishay Semiconductor & $40 \mathrm{~V}, 4 \mathrm{~A} \mathrm{~V}_{\mathrm{f}}=490 \mathrm{mV}$ \\
\hline Decoupling Capacitor & ECW-U1105KCV & Panasonic - ECG & $1 \mu \mathrm{F}, 100 \mathrm{~V}$, Polyethylene \\
\hline DSP & TMS320F2808 & Texas Instruments & \\
\hline Boost Capacitor & ECO-S1KA222BA & Panasonic & $2200 \mu \mathrm{F}, 80 \mathrm{~V}$ \\
\hline
\end{tabular}

A schematic and printed circuit board (PCB) was designed with Altium Designer 10 [25]. The PCB was populated and debugged in the lab and a photo of the prototype is shown in Figure 3-7. 


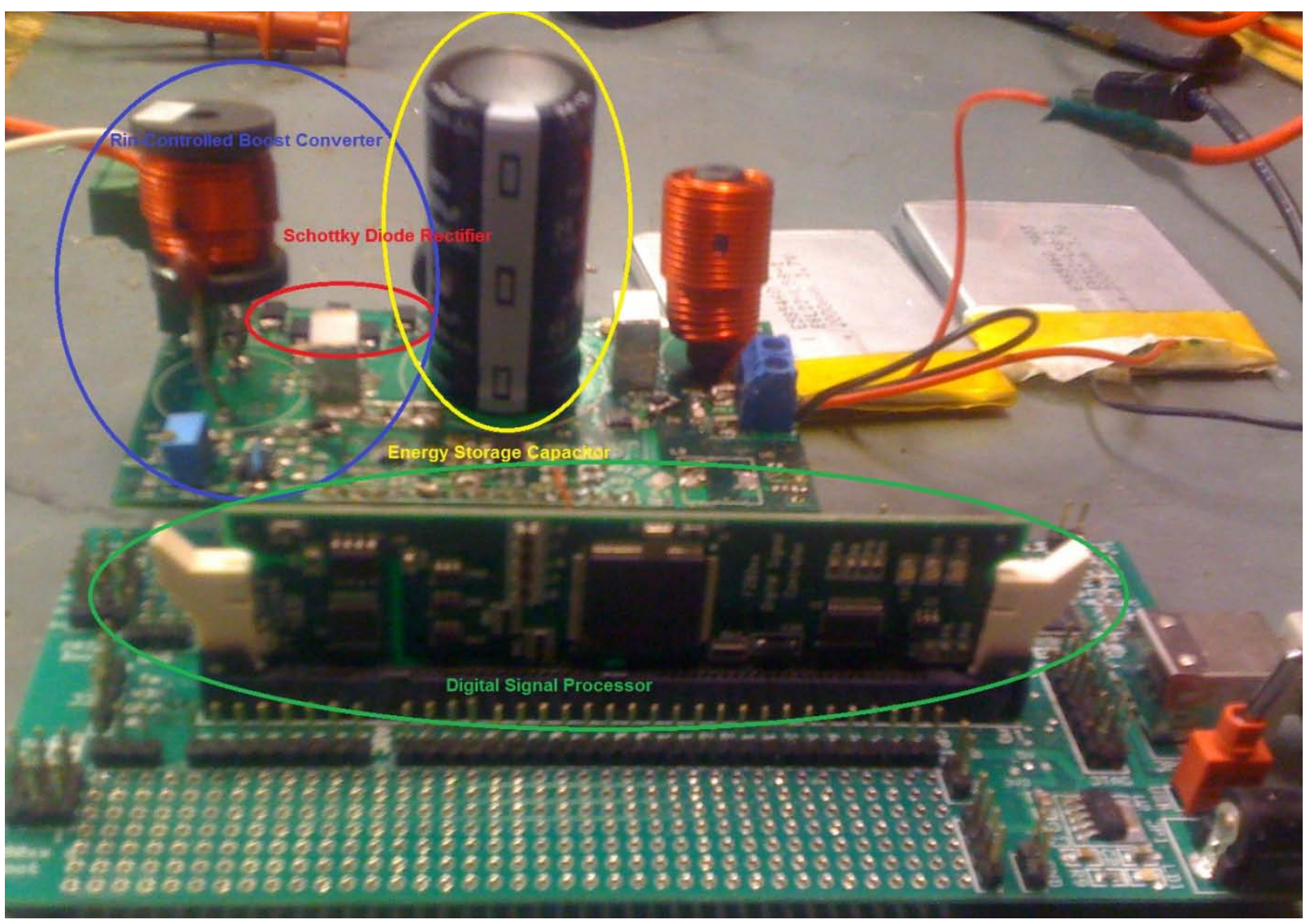

\section{Figure 3-7 - Printed Circuit Board Prototype of PEM}

The DSP is running at a clock frequency of $100 \mathrm{MHz}$ and driving the Boost converter at a switching frequency of $250 \mathrm{kHz}$ with the onboard ADC sampling each signal at $125 \mathrm{kHz}$. The $\mathrm{R}_{\mathrm{in}}$ Controlled Boost converter uses the sensed values of the rectified input voltage, $\mathrm{V}_{\text {rect }}$ as well as the output of the current sense amplifier sensing the inductor current, $\mathrm{I}_{\mathrm{boost}}$. The voltage of the ESC, $\mathrm{V}_{\text {boost }}$, is also sensed and is used only for overvoltage protection of the Boost converter. The voltage signals, $\mathrm{V}_{\text {rect }}$ and $\mathrm{V}_{\text {Boost }}$, are too large to be sampled directly by the ADC and need to be reduced in magnitude using a resistor divider, shown in Figure 3-8. The voltage divider will reduce the voltage level below the 3 Volt maximum voltage of the ADC input pins. The value for the resistors in the voltage divider can be calculated using equation (3.6) and (3.7), where $\mathrm{V}_{\text {sample }}$ is the peak value for 
the voltage that is to be sampled and $\mathrm{V}_{\mathrm{ADCmax}}$ is the maximum voltage level of the analog input pin on the ADC.

$$
\frac{V_{\text {sample }} \cdot R_{2}}{R_{1}+R_{2}}=V_{A D C \max }
$$

Equation (3.6) can be rearranged to solve for $\mathrm{R}_{1}$, shown in equation (3.7)

$$
R_{2}\left(\frac{V_{\text {sample }}}{V_{A D C \max }}-1\right)=R_{1}
$$

Table 3-2 has been provided for the value of the voltage divider resistors that were calculated and used in the prototype as shown in Figure 3-8, a nominal value of $10 \mathrm{k} \Omega$ has been selected for $\mathrm{R}_{2}$ and $\mathrm{R}_{4}$.

Table 3-2 - Table of Values used for Voltage Dividers

\begin{tabular}{|c|c|}
\hline Resistor & Value \\
\hline $\mathrm{R}_{1}$ & $133 \mathrm{k} \Omega$ \\
\hline $\mathrm{R}_{2}$ & $10 \mathrm{k} \Omega$ \\
\hline $\mathrm{R}_{3}$ & $267 \mathrm{k} \Omega$ \\
\hline $\mathrm{R}_{4}$ & $10 \mathrm{k} \Omega$ \\
\hline
\end{tabular}

The CSA is required to amplify the differential voltage across the current sense resistor in order to increase the resolution of the sensed inductor current. The gain of the CSA is selected to be 4.7 $\mathrm{V} / \mathrm{V}$. The sensed value for the inductor current is used as the feedback signal for the Boost Average Current Controller, while the sensed $\mathrm{V}_{\text {rect }}$ is used by the three different control modes to calculate the reference current value. 


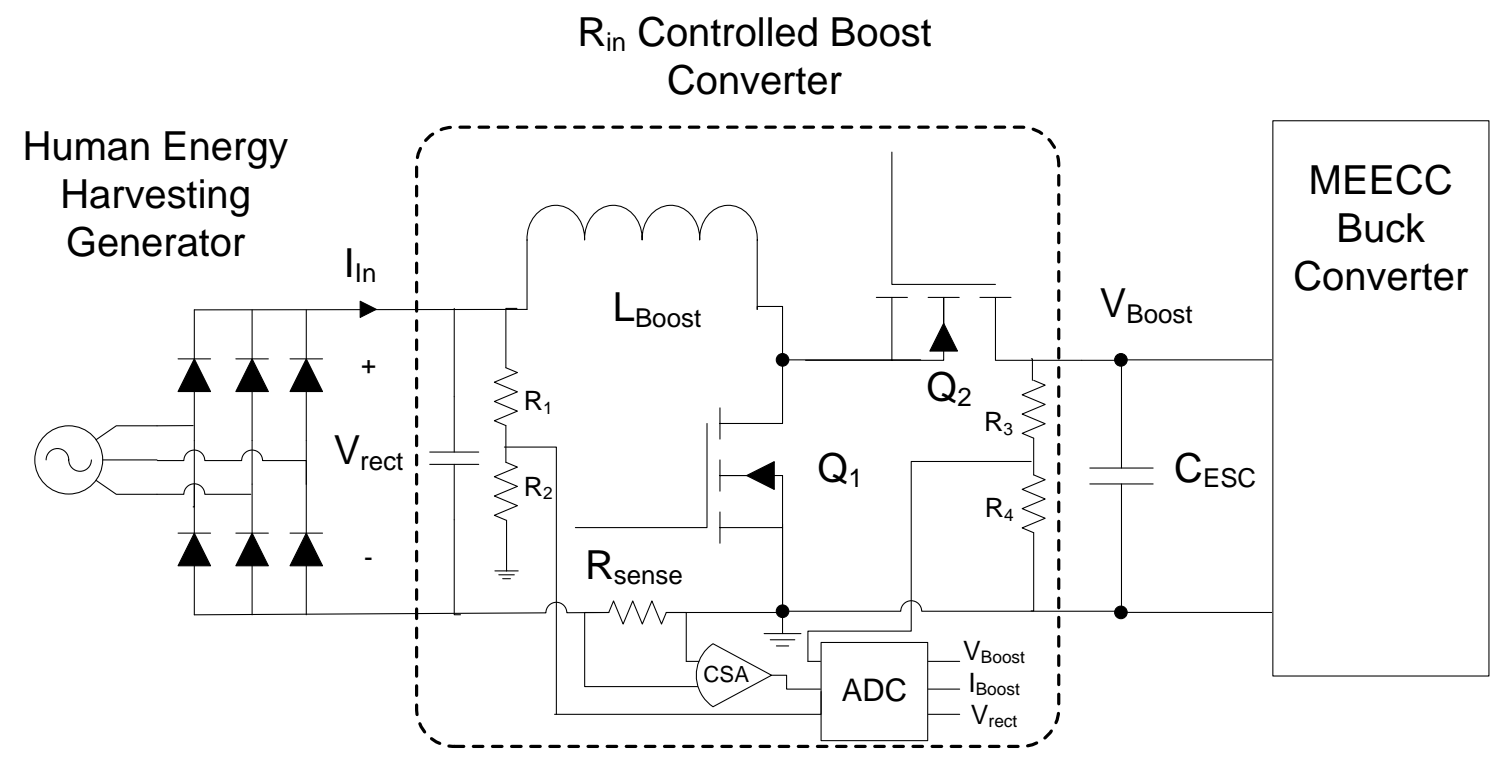

Figure 3-8 - Schematic of $\mathbf{R}_{\text {in }}$ Controlled Boost Converter with ADC Connections

Both the reference current and the digital controller for the Boost Average Current Controller are calculated at the same frequency as the ADC at $125 \mathrm{kHz}$. The derivation and design of the compensator will be covered in the next sections.

\subsection{Digital Average Current Mode Controlled Boost Converter Plant Transfer Function}

This section will cover the derivation of the small signal transfer function of the average current mode controlled Boost converter in the s-domain. The transfer function will then be discretized with zero-order-hold including the effects of sampling and hold and computational delay. With the discretized transfer function a suitable controller can be selected along with the discreized equations used to represent the digital controller. 
The small signal model of the Boost converter operating in CCM, shown in Figure 3-9, can be determined by averaging the two sets of differential equations that represent the two operating states of the Boost converter similar to [26].

\section{Boost Converter Operating in CCM}

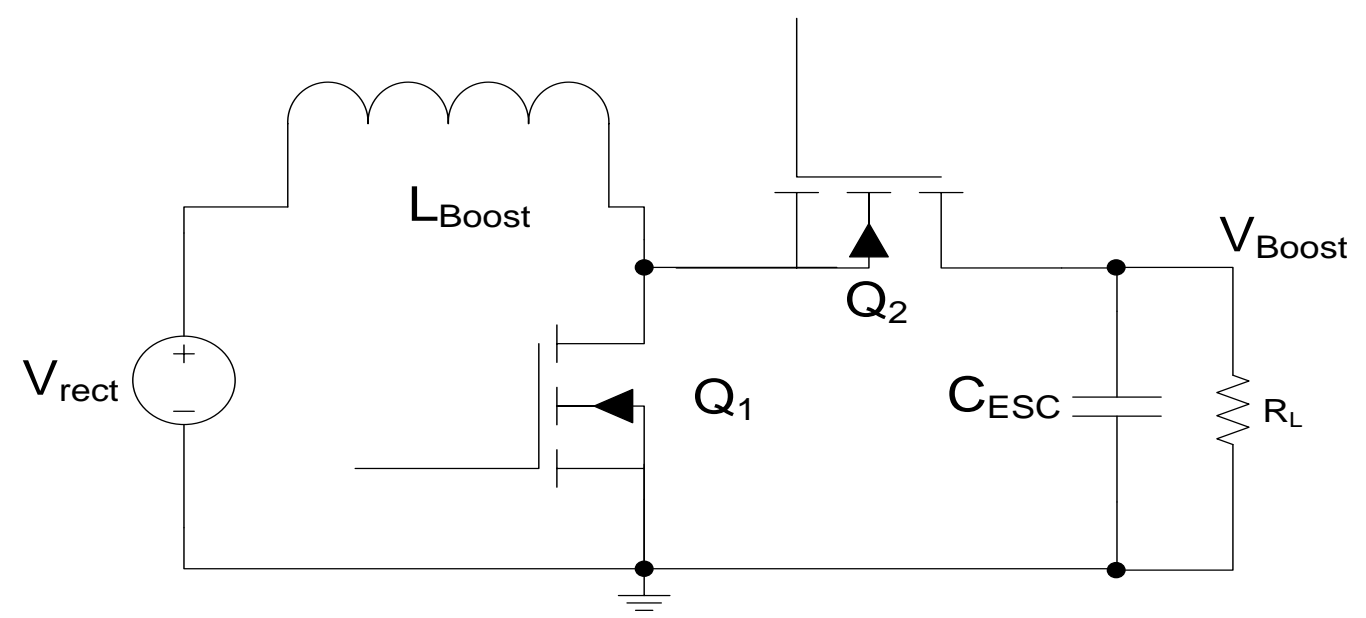

Figure 3-9 - Schematic of Boost Converter Operating in CCM

The first operating state of the Boost converter is when switch Q1 is conducting current and switch

Q2 is open as observed in Figure 3-10. The time that the boost converter is operating in this state is equal to the duty ratio, $\mathrm{D}$. 


\section{Boost Converter \\ Operating State 1}

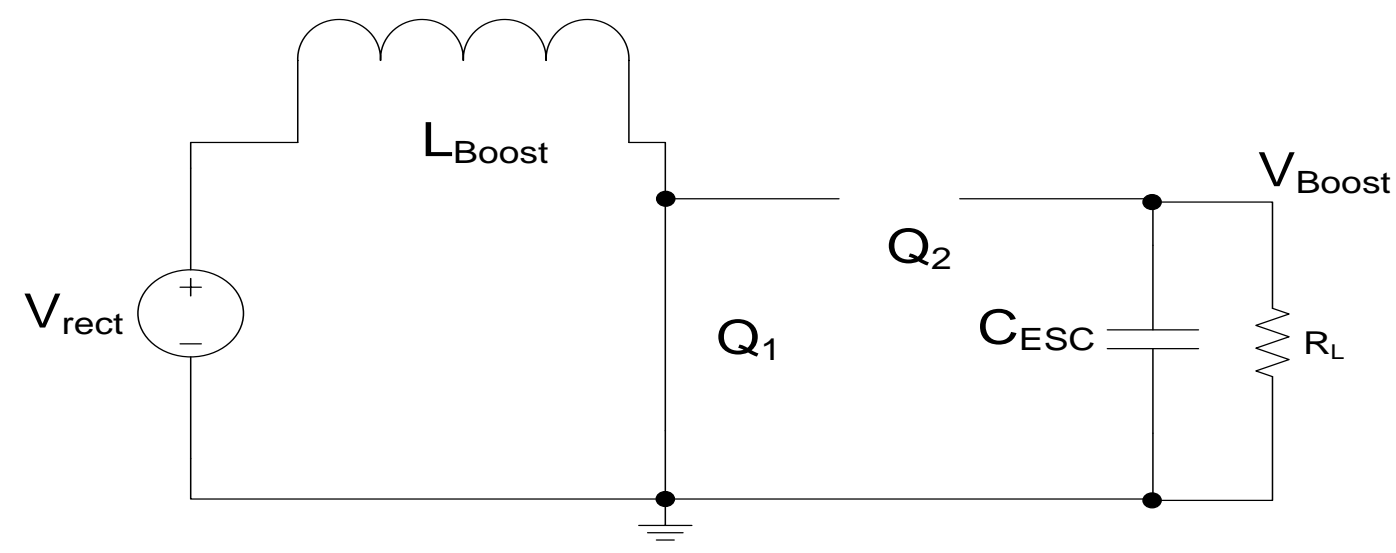

Figure 3-10 - Boost Converter in Operating State 1

The differential equations for operating state 1 can be written as shown in equations (3.8) and (3.9)

$$
\begin{aligned}
\frac{d i_{L}}{d t} & =\frac{V_{\text {rect }}}{L_{\text {Boost }}} \\
\frac{d V_{\text {Boost }}}{d t} & =-\frac{V_{\text {Boost }}}{R_{L} C_{E S C}}
\end{aligned}
$$

The second operating state of the Boost converter is when switch Q1 is open and switch Q2 is conducting current as observed in Figure 3-11. The time that the Boost converter is operating in this state is equal to the complementary of the duty cycle, 1-D. 


\section{Boost Converter \\ Operating State 2}

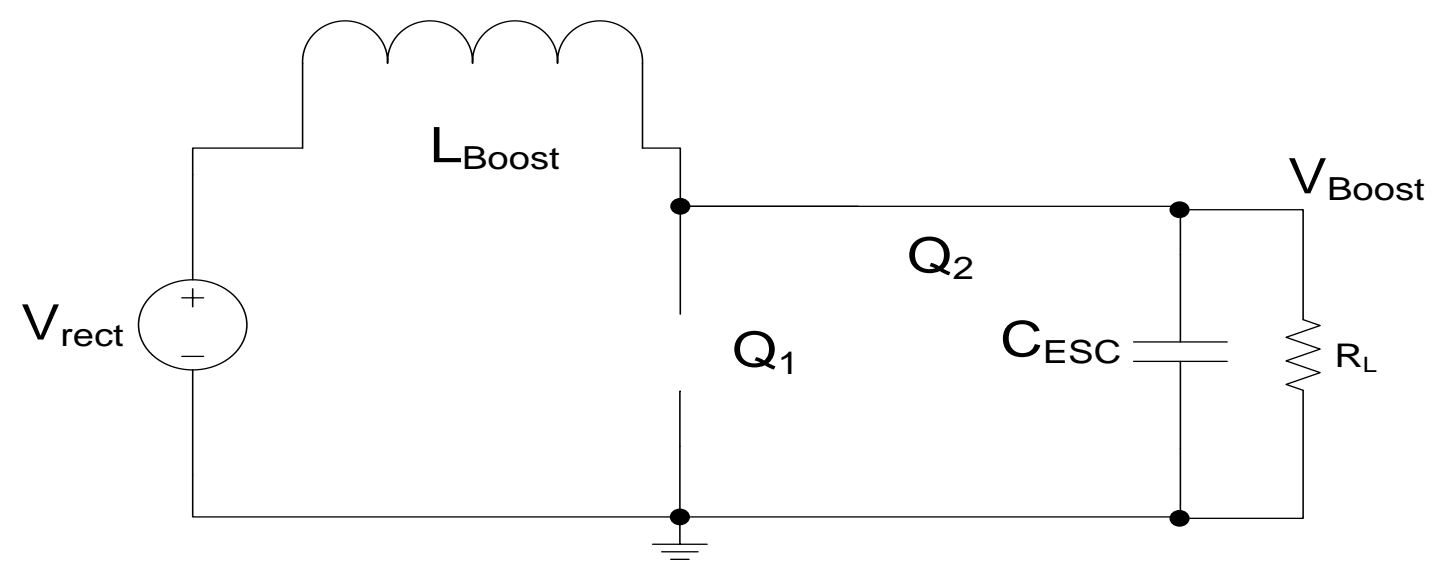

Figure 3-11 - Boost Converter in Operating State 2

The differential equations for operating state 2 can be expressed as shown in equations (3.10) and

$$
\begin{gathered}
\frac{d i_{L}}{d t}=\frac{V_{\text {rect }}-V_{\text {Boost }}}{L_{\text {Boost }}} \\
\frac{d V_{\text {Boost }}}{d t}=\frac{i_{L}}{C_{E S C}}-\frac{V_{\text {Boost }}}{R_{L} C_{E S C}}
\end{gathered}
$$

The equations (3.8-3.11) from both states can be added together using the average time in each state as defined by the duty ratio, and are given in (3.12) and (3.13)

$$
\begin{gathered}
\frac{d i_{L}}{d t}=\frac{V_{\text {rect }}-(1-D) V_{\text {Boost }}}{L_{\text {Boost }}} \\
\frac{d V_{\text {Boost }}}{d t}=\frac{(1-D) i_{L}}{C_{E S C}}-\frac{V_{\text {Boost }}}{R_{L} C_{E S C}}
\end{gathered}
$$

The control parameters, input and output variables are all replaced with a steady-state DC value and a small time-varying component, shown in (3.14) and (3.15) 


$$
\begin{gathered}
\frac{d\left(I_{L}+\hat{i}_{L}\right)}{d t}=\frac{V_{\text {rect }}+\hat{v}_{\text {rect }}-(1-(D+\hat{d}))\left(V_{\text {Boost }}+\hat{v}_{\text {Boost }}\right)}{L_{\text {Boost }}} \\
\frac{d\left(V_{\text {Boost }}+\hat{v}_{\text {Boost }}\right)}{d t}=\frac{(1-(D+\hat{d}))\left(I_{L}+\hat{i}_{L}\right)}{C_{E S C}}-\frac{\left(V_{\text {Boost }}+\hat{v}_{\text {Boost }}\right)}{R_{L} C_{E S C}}
\end{gathered}
$$

After expanding the terms and neglecting the small-signal products and DC products, the equations in (3.14) and (3.15) can be rewritten in terms of the small-signals, shown in (3.16) and (3.17)

$$
\begin{gathered}
\frac{d \hat{i}_{L}}{d t}=\frac{\hat{d} V_{\text {Boost }}+\hat{v}_{\text {rect }}-(1-D) \hat{v}_{\text {Boost }}}{L_{\text {Boost }}} \\
\frac{d \hat{v}_{\text {Boost }}}{d t}=\frac{(1-D) \hat{i}_{L}-\hat{d} I_{L}}{C_{E S C}}-\frac{\hat{v}_{\text {Boost }}}{R_{L} C_{E S C}}
\end{gathered}
$$

The linearized block diagram of the Boost converter can be created using (3.16) and (3.17) and simplified using block diagram simplification techniques as illustrated in Figure 3-12. 

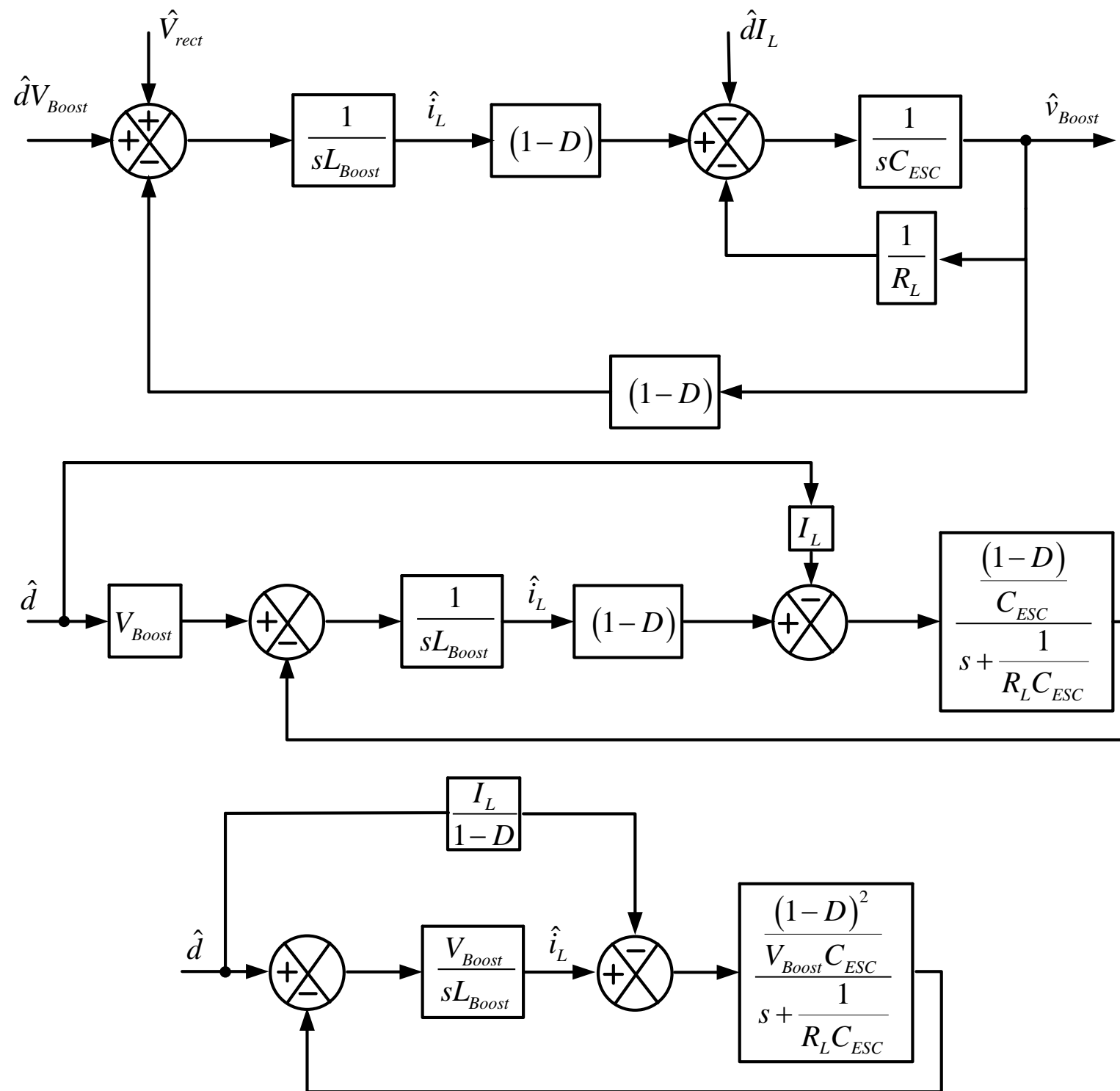

$$
\stackrel{\hat{d}}{\longrightarrow} \frac{V_{\text {Boost }} C_{E S C} s+2(1-D) I_{L}}{L_{\text {Boost }} C_{E S C} s^{2}+\frac{L_{\text {Boost }}}{R_{L}} s+(1-D)^{2}} \stackrel{\hat{i}_{L}}{\longrightarrow}
$$

Figure 3-12 - Simplification of Linearized Block Diagram of Boost Converter operating in CCM 
From the block diagram simplification of the small signal transfer function from duty cycle to inductor current, the continuous time power stage model in the s-domain, $G_{p B o o s t}(s)$ can be determined as shown in (3.18)

$$
G_{p \text { Boost }}(s)=\frac{\hat{i_{L}}}{\hat{d}}=\frac{V_{\text {Boost }} C_{E S C} s+2(1-D) I_{L}}{L_{\text {Boost }} C_{E S C} s^{2}+\frac{L_{\text {Boost }}}{R_{L}} s+(1-D)^{2}}
$$

As the compensator will be designed in the digital domain using the direct digital design approach, the continuous time power stage model along with the components that make up the closed loop digital system will first need to be discretized [27]. The closed loop digital control system including the effect of sample and hold is shown in Figure 3-13.

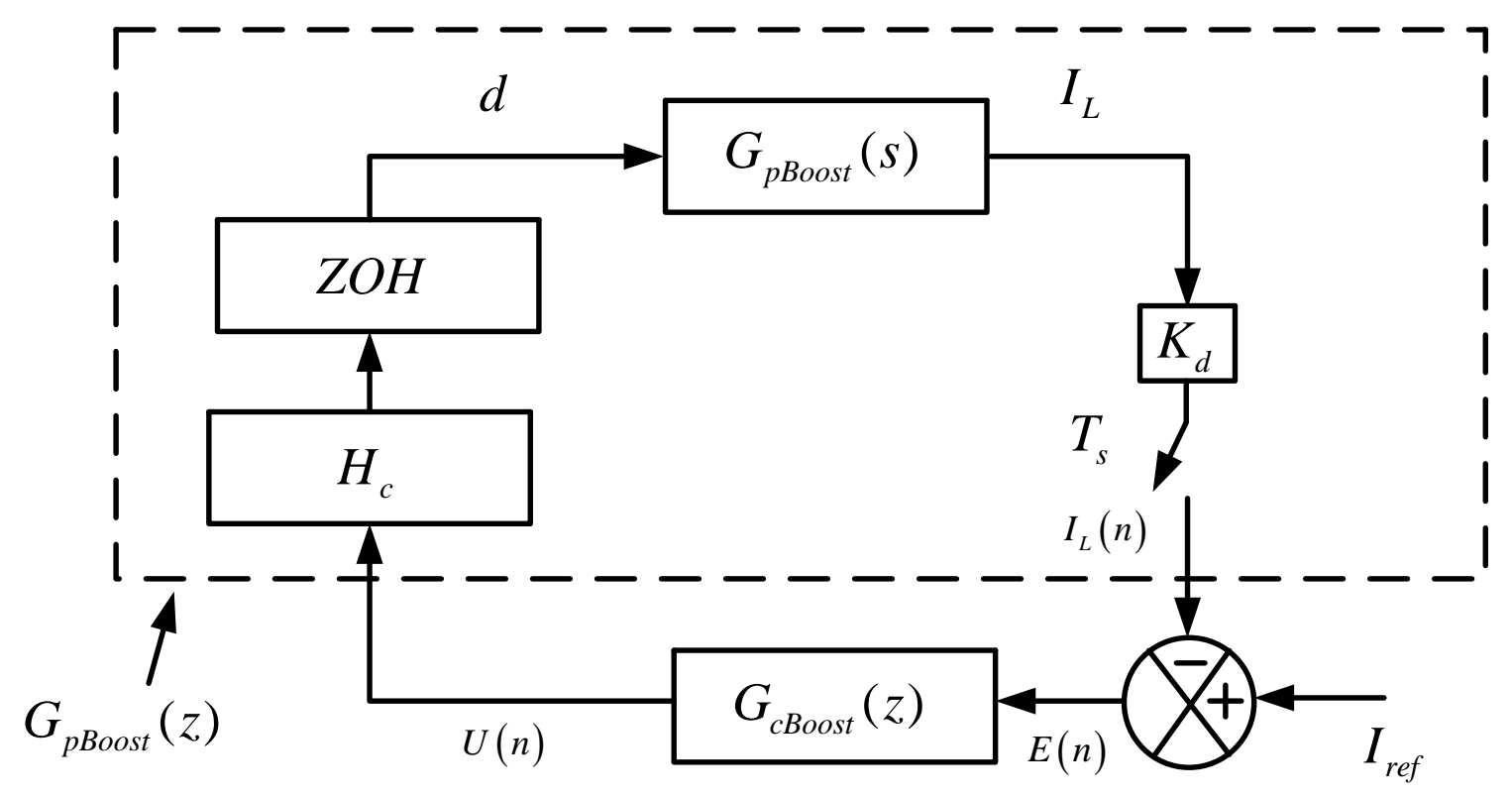

Figure 3-13 - Closed Loop Block Diagram of Digital Control System [28]

The sampling of the feedback signal by the ADC is represented by an ideal sampler with a time period of $T_{s}$. The gain of the ADC along with the gain of the sensing network is taken into account in the block $\mathrm{K}_{\mathrm{d}}$. The digital representation of the sampled feedback signal is $\mathrm{I}_{\mathrm{L}}(\mathrm{n})$. The sampling 
effect of the ADC and the holding effect of the PWM creates a sample and hold device, which can be represented by a zero-order-hold $(\mathrm{ZOH})$ with a time delay of $\mathrm{T}_{\mathrm{s}} / 2$. The s-domain transfer function of the sample and hold device can be represented by (3.19),[29].

$$
S H(s)=\frac{1-e^{-s T_{s}}}{s}
$$

The time delay from the sampling instant to the time when the PWM duty ratio is updated is represented by the computational delay block $\mathrm{H}_{\mathrm{c}}$. This block will add a time delay of $\mathrm{T}_{\mathrm{d}}$ into the control loop and will take into account the conversion time of the ADC as well as the computational time for the calculation of the digital controller. The transfer function for $\mathrm{H}_{\mathrm{c}}$ is shown in (3.20), [29].

$$
H_{c}(s)=e^{-s T_{d}}
$$

The discrete-time transfer function of the converter plant $\mathrm{G}_{\mathrm{pBoost}}(\mathrm{z})$ shown in Figure 3-13, is the discretization of the s-domain plant model with $\mathrm{ZOH}$. The plant model includes the sampler, the current sensing network gain $\mathrm{K}_{\mathrm{d}}$ and the time delay from sampling instant to the update of the duty cycle and is given in (3.21), where $\mathrm{Z}$ denotes the z-transform of the transfer function inside the parenthesis [], [27].

$$
G_{p \text { Boost }}(z)=Z\left[\frac{1}{s}\left(1-e^{-s T_{s}}\right) \cdot H_{c}(s) \cdot G_{p \text { Boost }}(s) \cdot K_{d}\right]
$$

Matlab was used to calculate the z-transform of the above transfer function to determine the number of poles and zeros of the transfer function to determine the order of compensator needed to 
compensate the system. It was found that the order of the system changed with the computation delay of the system, $T_{d}$. When $T_{d}$ is equal to 0 , the order of $G_{p B o o s t}(z)$ remained the same, when $0<T_{d}$ $<\mathrm{T}_{\mathrm{s}}$, the number of poles and zeros of $\mathrm{G}_{\mathrm{pBoost}}(\mathrm{z})$ each increased by 1 . The time delay from the sample instant to when the PWM is updated will be calculated in Section 5.2. For the purposes of determining the number of poles and zeros of the system, a delay of less than $\mathrm{T}_{\mathrm{s}}$ was used. Based on (3.18) and the additional pole and zero created by the time delay, $\mathrm{T}_{\mathrm{d}}$, the system has three poles and two zeros.

As the plant, $G_{p B o o s t}(z)$ to be compensated has three poles and two zeros, we have selected a digital two-pole two-zero compensator to compensate the $\mathrm{R}_{\mathrm{in}}$ Controlled Boost converter. The digital compensator, $G_{c B o o s t}(z)$ is given by equation (3.22), where $B_{2}, B_{1}, B_{0}, A_{2}$ and $A_{1}$ are the controller coefficients, $\mathrm{U}$ is the controller output and $\mathrm{E}$ is the error signal[28].

$$
G_{\text {cBoost }}(z)=\frac{U}{E}=\frac{B_{2} z^{2}+B_{1} z+B_{0}}{-A_{2} z^{2}-A_{1} z+1}
$$

The controller can be written in discrete form as shown in (3.23), where the quantities with a (n) denote the current sampling cycle, the quantities with a (n-1) denote one sample old values and so on.

$$
U(n)=A_{1} \cdot U(n-1)+A_{2} \cdot U(n-2)+B_{0} \cdot E(n)+B_{1} \cdot E(n-1)+B_{2} \cdot E(n-2)
$$

The digital two-pole two-zero compensator can be directly designed in the z-domain using Matlab’s sisotool using the direct digital design approach similar to [28]. By directly designing the compensator in the digital domain the poles and zeros can be located directly which results in a better phase margin and bandwidth for the power converter. The compensator can be entered directly into the DSP using the discretized equation (3.23). 


\subsection{Boost Two-Pole Two-Zero Compensator with Voltage Adaptive Compensator}

Gain

In a conventional PFC Boost converter, the output voltage is the control variable and is regulated by a voltage loop [23]. However, as previously mentioned there is no voltage loop to regulate the output voltage of the $\mathrm{R}_{\text {in }}$ Controlled Boost converter. This makes compensation of the current loop in the Boost converter difficult when using a conventional two-pole two-zero compensator because of the wide output voltage range and it is difficult to optimize the controller for all output voltage conditions. With a conventional two-pole two-zero compensator a compromise between low phase margin at high output voltage condition, and low bandwidth at low output voltage condition has to be made.

Using a conventional two-pole two-zero compensator, a controller was designed that can achieve $1.06 \mathrm{kHz}$ bandwidth and 86 degrees of phase margin at an output voltage, $\mathrm{V}_{\text {boost }}$, of 10 Volts and $11.8 \mathrm{kHz}$ and 79.3 degrees of phase margin at 80 volts output, $\mathrm{V}_{\text {boost }}$. The open loop bode plot of the Boost current loop at different output voltages with the conventional two-pole two-zero compensator is shown in Figure 3-14. 


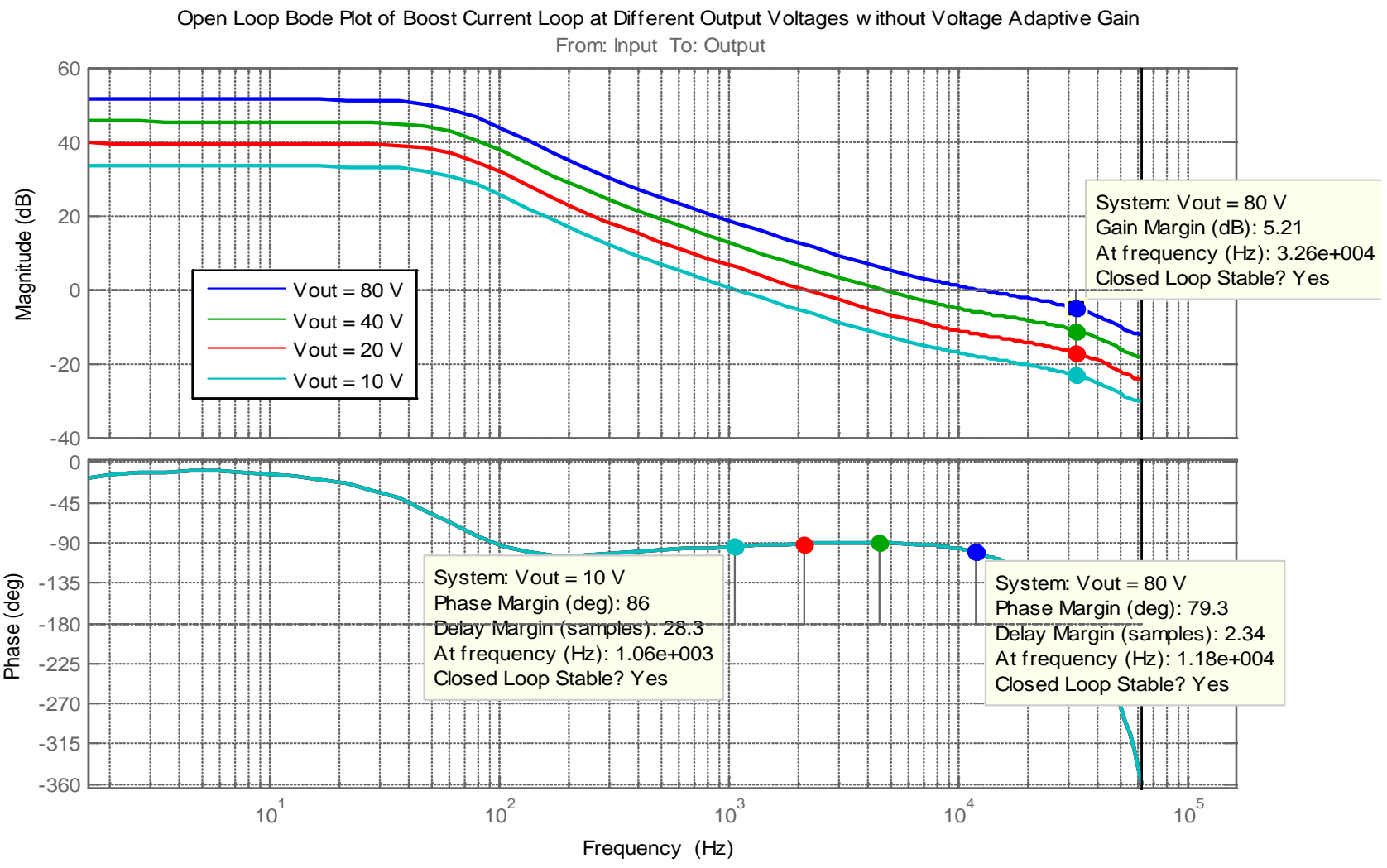

Figure 3-14 - Open Loop Bode Plot of Boost Current Loop at Different Output Voltages with a Conventional Two-pole Two-zero Compensator

As can be observed in Figure 3-14, the transfer functions are all different for the varying output voltages. With a wide output voltage range and while using a conventional two-pole two-zero compensator, the achievable bandwidth is $1.06 \mathrm{kHz}$ with 86 degrees of phase margin at an output voltage of 10 Volts, and $11.8 \mathrm{kHz}$ and 79.3 degrees of phase margin at 80 volts output. Thus it is difficult to design a controller to optimize both the low output voltage (10 Volts) and high output voltage (80 Volts) conditions. The compensator must be designed with a compromise between low bandwidth at low output voltage conditions and less phase margin at high output voltage conditions. Out of these two parameters it is more important to have a stable system than to have 
high bandwidth. Therefore we sacrifice bandwidth at the low output voltage range for system stability.

The low bandwidth at low output voltage condition will affect how quickly the Boost converter can respond to a step input change. In our case this step change comes in the form of a step input current command. When the Boost converter turns on the current must increase from 0 to the controlled value. The duration that the $\mathrm{R}_{\text {in }}$ Controlled Boost converter will be extracting power from the generator is typically between 200 - 400 ms for each gait cycle. If the Boost converter turn on time is less than $2 \mathrm{~ms}$ or $1 \%$ of the power harvesting period, the turn on time will be fast enough. A faster turn on time would only marginally increase the power extracted. In order to meet this turn on requirement, a higher bandwidth controller is required at the low output voltage condition. Just before the Boost converter has turned on the output voltage, $\mathrm{V}_{\text {boost }}$ will be at its lowest value which is the worst case scenario for the compensator bandwidth.

The $\mathrm{R}_{\text {in }}$ Controlled Boost converter using a conventional two-pole two-zero compensator was tested with a step input voltage, $\mathrm{V}_{\text {rect }}=0-5 \mathrm{~V}$ with $\mathrm{V}_{\text {Boost }}=9 \mathrm{~V}$ to determine the turn on time of the converter and is shown in Figure 3-15. As can be observed from the figure, the time required for the inductor current to increase to the reference level from the start of the Control MOSFET duty cycle is measured at $13.2 \mathrm{~ms}$. 


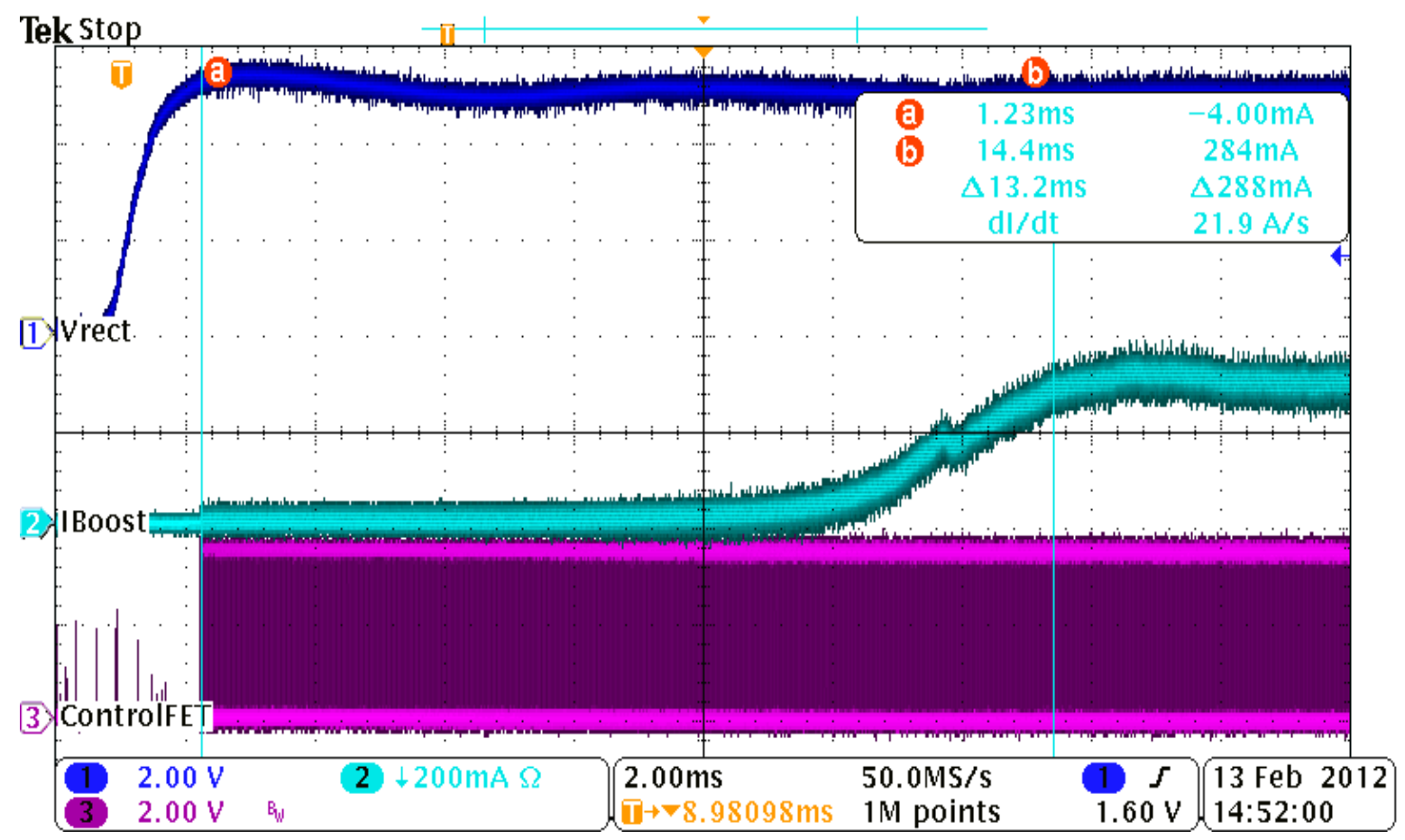

Figure 3-15 - Rise Time of Inductor Current for $\mathbf{R}_{\mathrm{in}}$ Controlled Boost Converter with Conventional Controller

In a situation where the converter is connected to the utility grid where the input power source is virtually unlimited and always present, this turn on delay would not be a large issue. As well as, the output voltage would typically be regulated by a PFC Boost converter in which case a higher bandwidth controller could be used. However, in the case of extracting power from human walking, the power source is not always present but rather turning on and off as fast as $2 \mathrm{~Hz}$ and available for only 200 - 400 ms per user gait cycle. A time delay of longer than 2 ms would reduce the amount of power that could be extracted from the generator much more significantly then with the utility grid. This time delay can be reduced by further increasing the bandwidth. However, this comes at the expense of a very sensitive and unstable compensator at higher output voltages. A much more elegant solution to this problem has been used. 
The small signal power stage model of the average current mode Boost converter, $G_{p B o o s t}(s)$, was derived in the s-domain in Section 3.6 and given by equation (3.18). The DC Gain of the transfer function can be extracted from (3.18) by substituting $s=0$ to get (3.24)

$$
\text { DC Gain }=\frac{2 \cdot I_{L}(1-D)}{(1-D)^{2}}
$$

The DC value of the inductor current is shown in (3.25)

$$
I_{L}=\frac{V_{\text {in }}}{R_{\text {in }}}
$$

The relationship between $\mathrm{V}_{\text {in }}$ and $\mathrm{V}_{\text {boost }}$, given by the duty cycle $\mathrm{D}$, is shown in (3.26)

$$
V_{\text {in }}=V_{\text {boost }}(1-D)
$$

After substituting (3.25) and (3.26) into (3.24) and some derivation, equation (3.27) can be derived.

$$
\text { DC Gain }=\frac{2 \cdot V_{\text {boost }}}{R_{\text {in }}}
$$

It can be observed from (3.27) that the DC gain of the Boost converter has a linear relationship with respect to $V_{\text {boost }}$ in the case of the $R_{\text {in }}$ Controlled Boost converter, while keeping $R_{\text {in }}$ constant. This varying DC gain is the cause for the poor control loop characteristics of the system over the operating range that was observed in Figure 3-14.

It is proposed in this thesis to use the $\mathrm{V}_{\text {boost }}$ voltage to modify the DC gain of the compensator to normalize the DC gain of the plant over the whole output voltage range of the Boost converter. The compensator with Voltage Adaptive Gain is written in discrete form given by equation (3.28), where $G_{a d p}$ is the adaptive gain of the compensator $B_{2}, B_{1}, B_{0}, A_{2}$ and $A_{1}$ are the controller coefficients, $\mathrm{U}$ is the controller output and $\mathrm{E}$ is the error signal.

$$
U(n)=A_{1} \cdot U(n-1)+A_{2} \cdot U(n-2)+G_{a d p}\left(B_{0} \cdot E(n)+B_{1} \cdot E(n-1)+B_{2} \cdot E(n-2)\right)
$$


The value of the Voltage Adaptive Gain that is required to normalize the plant is given by equation (3.29).

$$
G_{\text {adp }}=80 .\left(V_{\text {Boost }}\right)^{-1}
$$

In order to reduce the computational power that is required to run the Voltage Adaptive Gain compensator, the value of $\mathrm{G}_{\text {adp }}$ is updated at $1 \mathrm{kHz}$ or once every 125 cycles the compensator is calculated. The value of $G_{\text {adp }}$ is also quantized to four gain values which are all a power of two so that the DSP can achieve the multiplication with a logic shift left and further reduce the computational power that would be required for a full multiplication, shown in Table 3-3.

Table 3-3 - Quantized Values for Voltage Adaptive Gain

\begin{tabular}{|c|c|c|c|c|}
\hline & Gain Value & Power of 2 & Logical Shift Left Value & Condition \\
\hline $\mathrm{G}_{\text {adp }}=$ & 8 & $2^{3}$ & 3 & $V_{\text {Boost }} \leq 10 \mathrm{~V}$ \\
\hline $\mathrm{G}_{\text {adp }}=$ & 4 & $2^{2}$ & 2 & $10 \mathrm{~V}<V_{\text {Boost }} \leq 20 \mathrm{~V}$ \\
\hline $\mathrm{G}_{\text {adp }}=$ & 2 & $2^{1}$ & 1 & $20 \mathrm{~V}<V_{\text {Boost }} \leq 40 \mathrm{~V}$ \\
\hline $\mathrm{G}_{\text {adp }}=$ & 1 & $2^{0}$ & 0 & $40 \mathrm{~V}<V_{\text {Boost }}$ \\
\hline
\end{tabular}

In order to determine that the update rate of $\mathrm{G}_{\text {adp }}$ is sufficient at $1 \mathrm{kHz}$, the slew rate if $\mathrm{V}_{\text {Boost }}$ is determined from experimental data. The test data used to calculate the slew rate of $\mathrm{V}_{\text {Boost }}$ is taken from the worst case scenario, when the average input power is highest and the output charging power to the battery is at its lowest value. This scenario will require the ESC to store the most amount of energy. The conditions used are with an average input power of $\mathrm{P}_{\text {in }}=15 \mathrm{~W}$ and an average output power limited by the battery $\mathrm{P}_{\text {battmin }}=12.8 \mathrm{~W}$. The slew rate of $\mathrm{V}_{\text {Boost }}$ is given in Figure 3-16, and it can be observed from the figure that the maximum value for the slew rate is 0.45 
$\mathrm{V} / \mathrm{ms}$. At this slew rate, $\mathrm{G}_{\text {adp }}$ will update 22 times during a change of $10 \mathrm{~V}$ of $\mathrm{V}_{\text {Boost }}$ before a recalculation of the Voltage Adaptive Gain, $G_{a d p}$ is required.

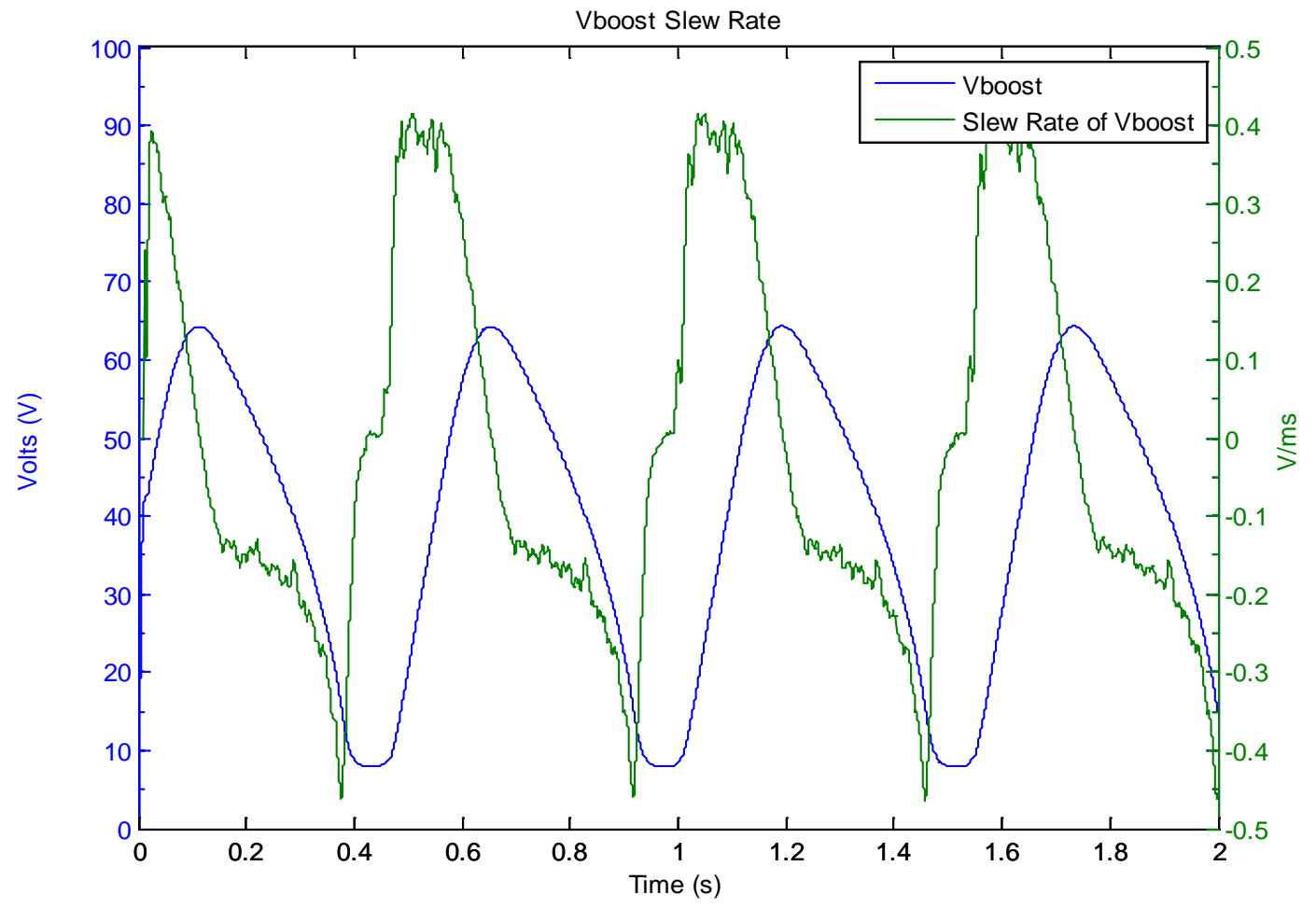

Figure 3-16 - Slew Rate of $V_{\text {Boost }}$

A controller is implemented with voltage adaptive compensator gain and is used to compensate the system. The controller is identical to the first controller used above other than the addition of the $\mathrm{G}_{\text {adp }}$ term that is used to cancel the effect of the varying DC gain of the plant. This new controller is applied to the same transfer functions $\mathrm{G}_{\mathrm{pBoost}}(\mathrm{z})$ as before to see the effect of the Voltage Adaptive Compensator gain on the transfer function and is shown in Figure 3-17. 


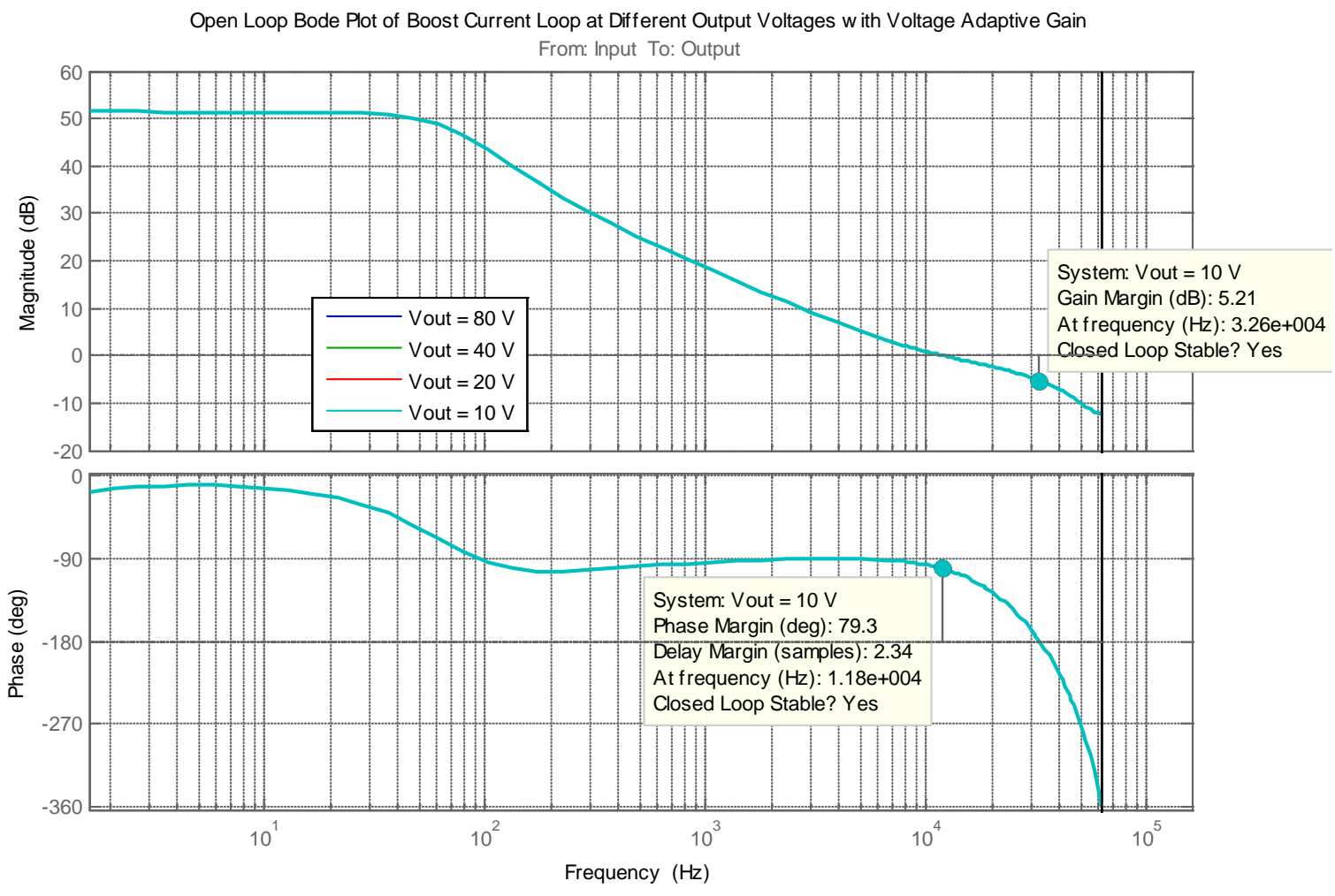

Figure 3-17 - Open Loop Bode Plot of Boost Current Loop at Different Output Voltages with a Voltage Adaptive Variable Gain Compensator

As can be observed in the figure above the bode plot for all four output voltage conditions have the same transfer function and performance characteristics. We can achieve the same performance at high and low output voltage conditions and there no longer needs to be a compromise with bandwidth at low output voltages and phase margin at high output voltages when using the voltage adaptive gain compensator. The new compensator can increase the bandwidth at $\mathrm{V}_{\text {boost }}=10 \mathrm{~V}$ from $1.06 \mathrm{kHz}$ to $11.8 \mathrm{kHz}$ with a phase margin of 79.3 degrees and with $\mathrm{V}_{\text {boost }}=80$ the bandwidth remains constant at $11.8 \mathrm{kHz}$ with a phase margin of 79.3 degrees. The new Voltage Aadaptive Gain two-pole two-zero compensator can achieve a turn on time of $660 \mu$ s under the same operating conditions as before with an input voltage step of $\mathrm{V}_{\text {rect }}=0-5 \mathrm{~V}, \mathrm{~V}_{\text {boost }}=9 \mathrm{~V}$ as shown in Figure 3-18. 
The previous turn on time without the Voltage Adaptive Gain compensator was 13.2 ms.

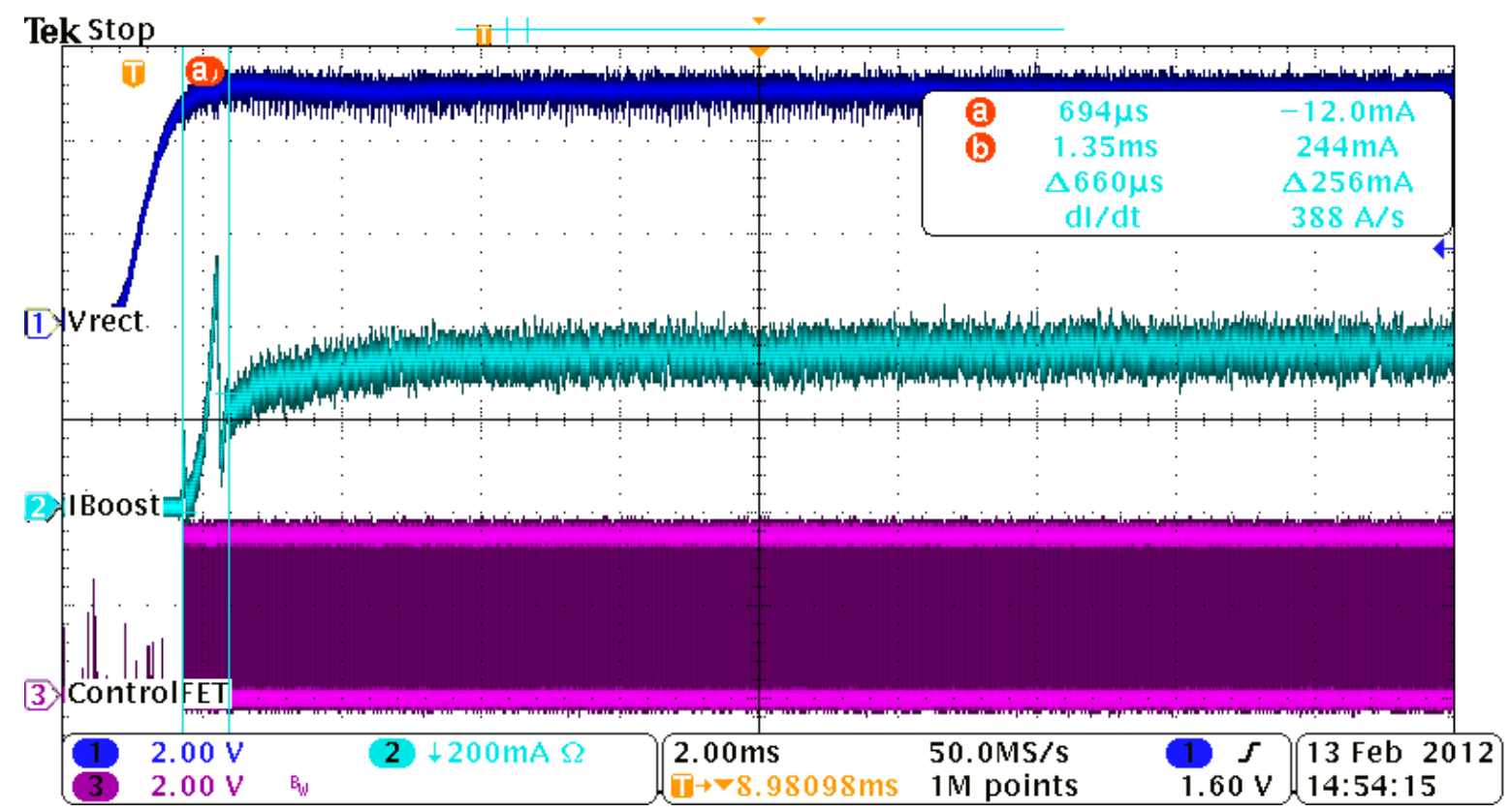

Figure 3-18 - Rise Time of Inductor Current for $\mathbf{R}_{\mathrm{in}}$ Controlled Boost Converter with Variable Gain Controller

It is common for an average current controlled converter to have a bandwidth of less than $1 / 10^{\text {th }}$ of the switching frequency [30]. In this case a bandwidth of $25 \mathrm{kHz}$ should be achievable with a switching frequency of $250 \mathrm{kHz}$. However, because the contributions of this thesis are in the control algorithms themselves and not on the dynamic response of the converter a current loop bandwidth of $11.8 \mathrm{kHz}$ will be sufficient for our needs. This allows us to sample at half the sampling frequency that would be needed in order to achieve the full $25 \mathrm{kHz}$ bandwidth having the benefit of reduced computational power to process the higher sampling frequency as well as processing the controllers. As the input voltage waveforms are relatively low frequency, a turn on time of less than $2 \mathrm{~ms}$ is acceptable, the bandwidth need not be further increased to reduce the turn on time and the additional stability that a lower bandwidth compensator possesses is more desirable for the control. 


\subsection{Experimental Verification and Discussion}

This section will show the $\mathrm{R}_{\text {in }}$ Controlled Boost converter prototype under the different controller operating conditions that were described in the previous section. To properly test the $\mathrm{R}_{\mathrm{in}}$ Controlled Boost converter prototype, an input voltage waveform is needed to provide a waveform profile that is similar to that which would be generated by the human energy harvester generator under normal walking conditions. An AC source with a diode in series is used to produce the half-wave rectified sinusoidal that is used as the input to the PEM, shown in Figure 3-19.

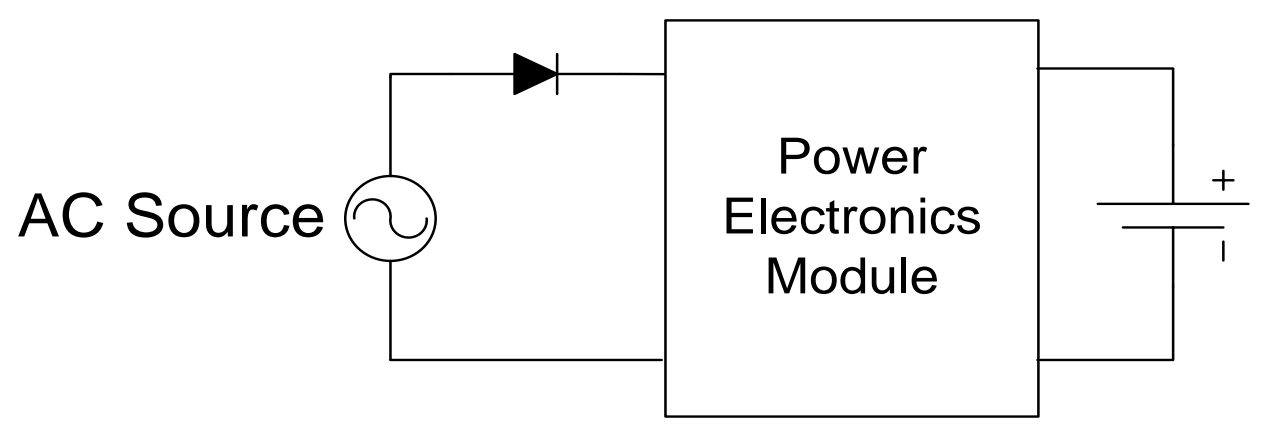

\section{Figure 3-19 - AC Input Source Schematic}

The comparison of the voltage produced by the energy harvester generator with a human walking at a typical walking speed of $1.5 \mathrm{~m} / \mathrm{s}$ and the voltage produced by the AC source with a diode in series is given in Figure 3-20. As can be observed in the figure, the half-wave rectified AC source provides a close approximation to the voltage produced by the human energy harvesting generator. The AC source is also able to remove any inconsistencies in the voltage profile that a human test subject would introduce as well as the ability to conduct experiments without the need of a human test subject to generate the electricity. 


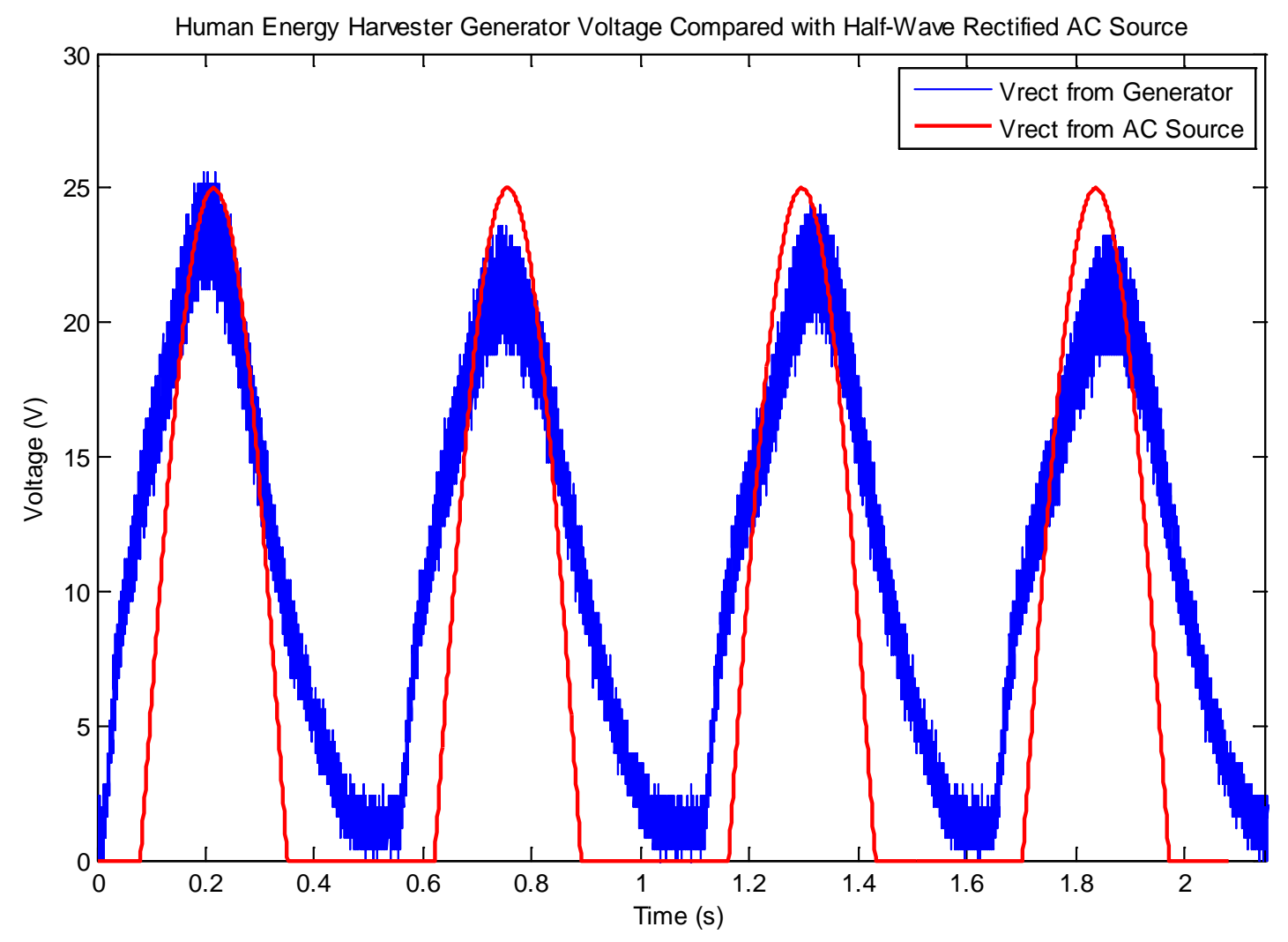

Figure 3-20 - Input Voltage Waveform Used to test Prototype

The first experimental waveform capture of the control modes is the Constant $\mathrm{R}_{\mathrm{in}}$ Control Mode shown in Figure 3-21 with the operating conditions, $V_{\text {in }}=30 \mathrm{~V}$ peak, $F_{\text {in }}=1,85 \mathrm{~Hz} \mathrm{I}_{\text {in }}=1.5$ A peak, and $\mathrm{R}_{\text {in }}=20 \Omega$. 


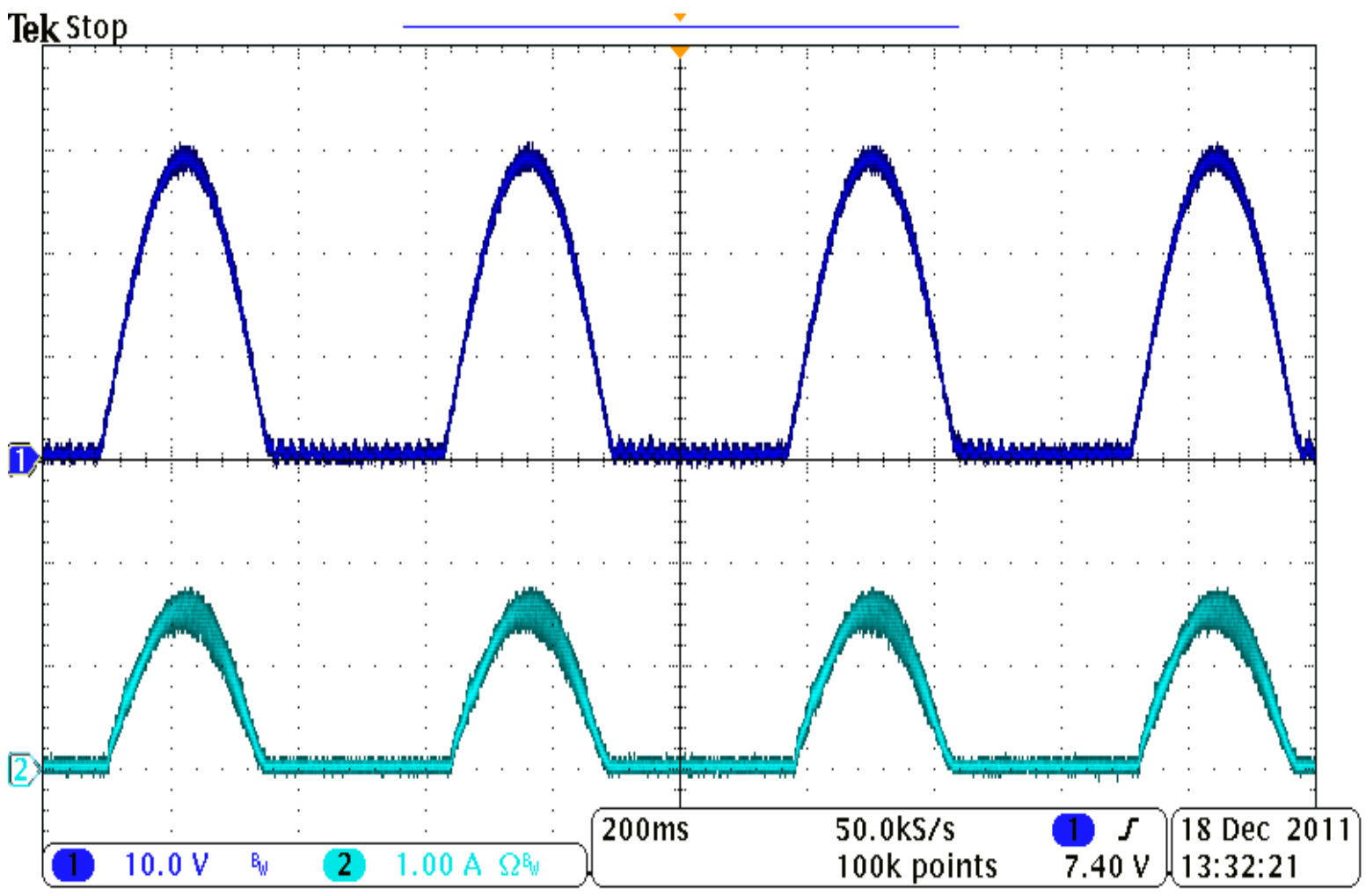

Figure 3-21 $-\mathbf{R}_{\text {in }}$ Controlled Boost Converter operating in Constant $\mathbf{R}_{\text {in }}$ Control Mode

Shown in the figure, is the input voltage, $\mathrm{V}_{\text {rect }}$ shown in waveform 1 , the input current, $\mathrm{I}_{\mathrm{boost}}$ shown in waveform 2. Looking at the oscilloscope waveforms captured of the Boost converter operating in Constant $\mathrm{R}_{\mathrm{in}}$ Control mode we can observe that the $\mathrm{I}_{\text {boost }}$ waveform is the same shape as the input voltage waveform $\mathrm{V}_{\text {rect }}$, and based on the waveforms the converter is emulating a constant resistive load as set by the user.

The next mode of operation is the Variable $\mathrm{R}_{\mathrm{in}}$ Control mode with the following operating conditions, $\mathrm{V}_{\text {in }}=20 \mathrm{~V}$ peak, $\mathrm{F}_{\text {in }}=1.85 \mathrm{~Hz}, \mathrm{I}_{\text {in }}=2$ A peak, $\mathrm{R}_{1}=\infty, \mathrm{R}_{2}=20 \Omega, \mathrm{R}_{3}=10 \Omega$. In this mode of control the emulated $\mathrm{R}_{\text {in }}$ can be set to different values depending on the level of the input voltage $\mathrm{V}_{\text {rect }}$ Shown in Figure 3-22, the converter is operating in Variable $\mathrm{R}_{\mathrm{in}}$ Control mode. Waveform 1 is the input voltage $\mathrm{V}_{\text {rect }}$ and waveform 2 is the input current, $\mathrm{I}_{\text {boost }}$. We can see from the waveforms that the input current, $\mathrm{I}_{\mathrm{boos}}$, is following the input voltage, $\mathrm{V}_{\text {rect }}$, similar to the previous case. 
However, there is a discontinuity when $\mathrm{V}_{\text {rect }}$ transitions above the voltage threshold level of $18 \mathrm{~V}$ and the emulated resistance transitions from $R_{2}$ to $R_{3}$. The input current, $I_{b o o s t}$ follows the profile of the $V_{\text {rect }}$ waveform with the new emulated resistor value, $R_{3}$ and returns to the previous emulated resistor value, $\mathrm{R}_{2}$ once the voltage drops below the $18 \mathrm{~V}$ threshold. There is also another voltage threshold that is being used to prevent the converter from drawing current when $\mathrm{V}_{\text {rect }}$ is too low, when the input voltage is below 3 Volts $\mathrm{R}_{1}$ is emulated which has a very high resistance value and therefore drawn no current.

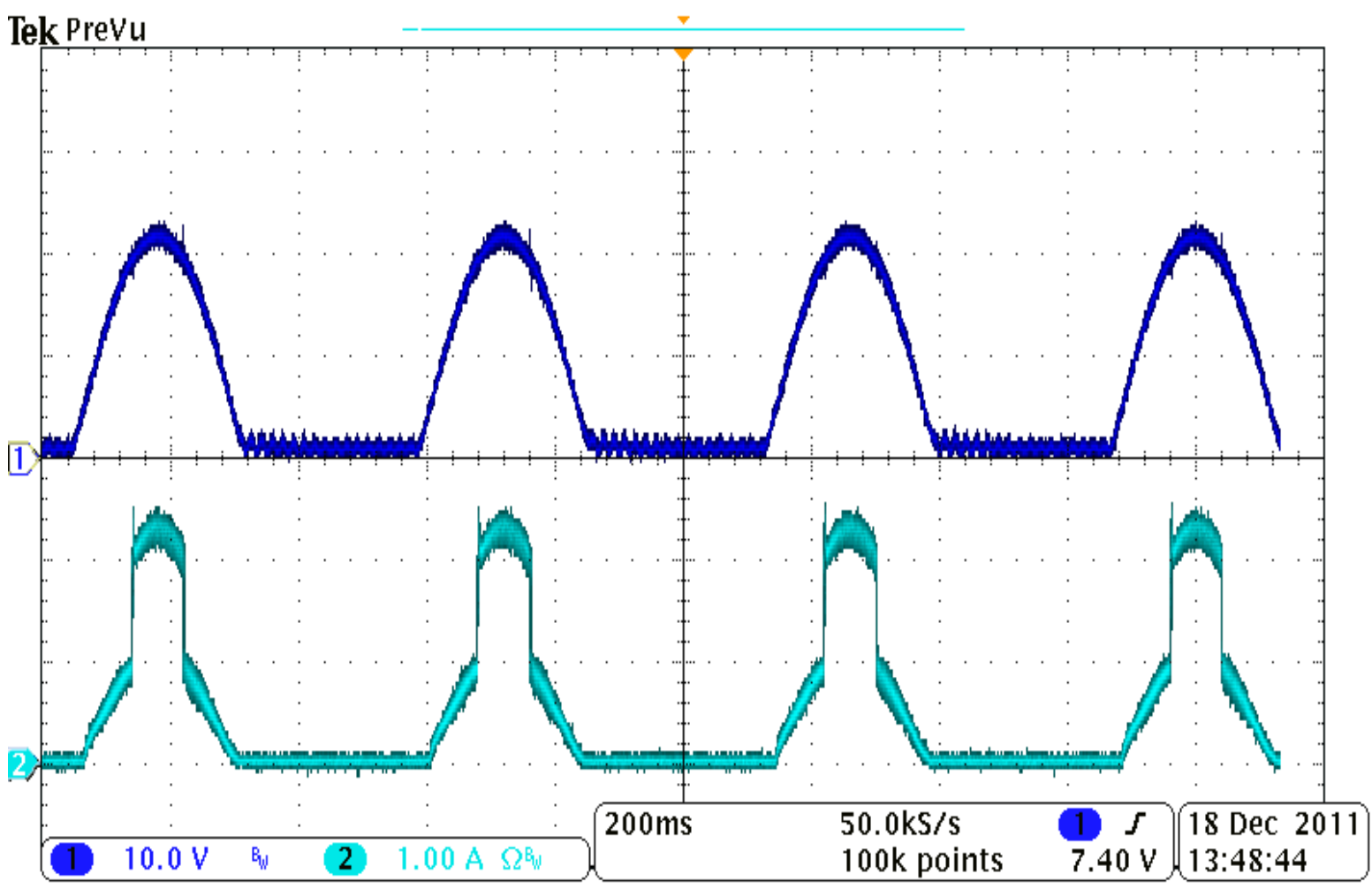

Figure 3-22 - Variable $\mathbf{R}_{\text {in }}$ Control Mode

From observing the waveforms of the Variable $\mathrm{R}_{\mathrm{in}}$ control mode we can see that the controller is emulating three separate resistor values depending on the input voltage. 
The next mode of control is the Dynamic $\mathrm{R}_{\text {in }}$ Control mode shown in Figure 3-23 with the following operating conditions, $\mathrm{V}_{\text {in }}=10 \mathrm{~V}$ peak, $\mathrm{F}_{\text {in }}=1.85 \mathrm{~Hz}, \mathrm{I}_{\mathrm{in}}=1$ A peak. This figure shows the input voltage in waveform $1, \mathrm{I}_{\text {boost }}$ as waveform 2 , and $\mathrm{R}_{\text {in }}$ as waveform $3 . \mathrm{R}_{\mathrm{in}}$ is the signal that the DSP uses to determine the dynamic resistor value to emulate. It can be observed in the figure that the input current follows the inverse of the input sinusoid $R_{\text {in }}$ value, as the voltage on the $R_{\text {in }}$ pin increases the value of the emulated resistor value decreases, drawing more power. The inverse of the $\mathrm{R}_{\text {in }}$ pin was chosen for ease of implementation. The current waveform also shows that the Dynamic $\mathrm{R}_{\mathrm{in}}$ Control can draw current in a discontinuous profile. The current will turn on after the voltage goes over a threshold value and turn off once it decreases below a different threshold value.

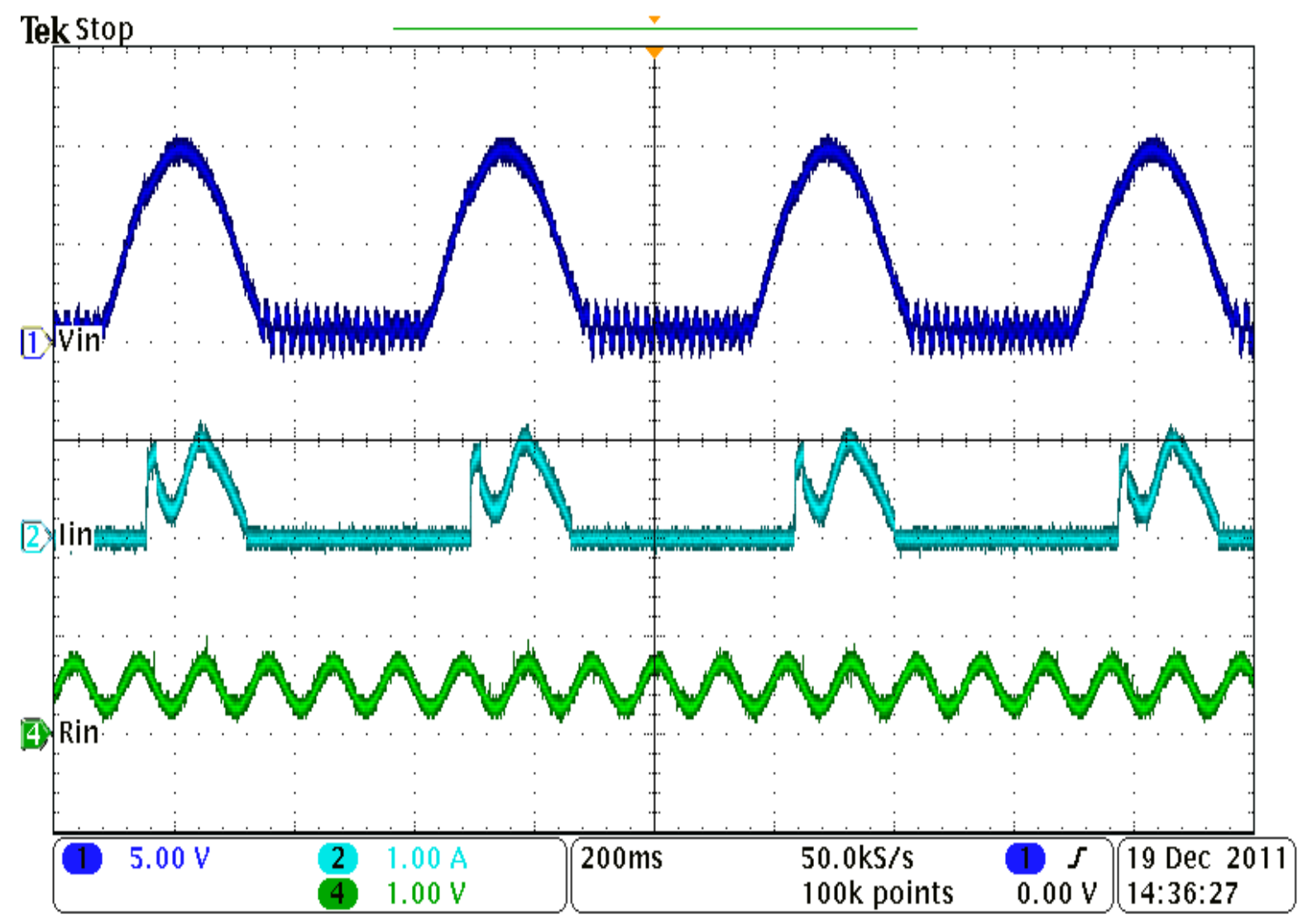

Figure 3-23 - Dynamic $\mathbf{R}_{\text {in }}$ Control Mode 
From the waveforms shown in Figure 3-23 we can confirm that the Dynamic $\mathrm{R}_{\text {in }}$ Controller is emulating the resistor value based on the voltage level on the $\mathrm{R}_{\mathrm{in}}$ pin of the ADC.

The DC efficiency of the Boost converter was measured operating in Constant $\mathrm{R}_{\text {in }}$ control mode and is shown in Figure 3-24. The efficiency is measured from input power to the diode rectifier to the output power of the Boost converter with the power level $P_{\text {in }}$ based on $V_{\text {in }}$ and $R_{\text {in. }}$. The input voltage of the Boost converter is $\mathrm{V}_{\text {in }}=20 \mathrm{~V}$ DC, the output voltage of the Boost converter is controlled by the MEECC control scheme of the Buck converter and is shown in red in Figure 3-24. The output current is changed by the Buck MEECC controller to regulate the output voltage of the Boost converter, $\mathrm{V}_{\text {Boost. }}$ The emulated input resistor $\mathrm{R}_{\text {in }}$ is changed to vary the power level and used to measure the different efficiencies of the Boost converter with the peak efficiency equal to 93\% with $\mathrm{R}_{\text {in }}=40 \Omega$. 


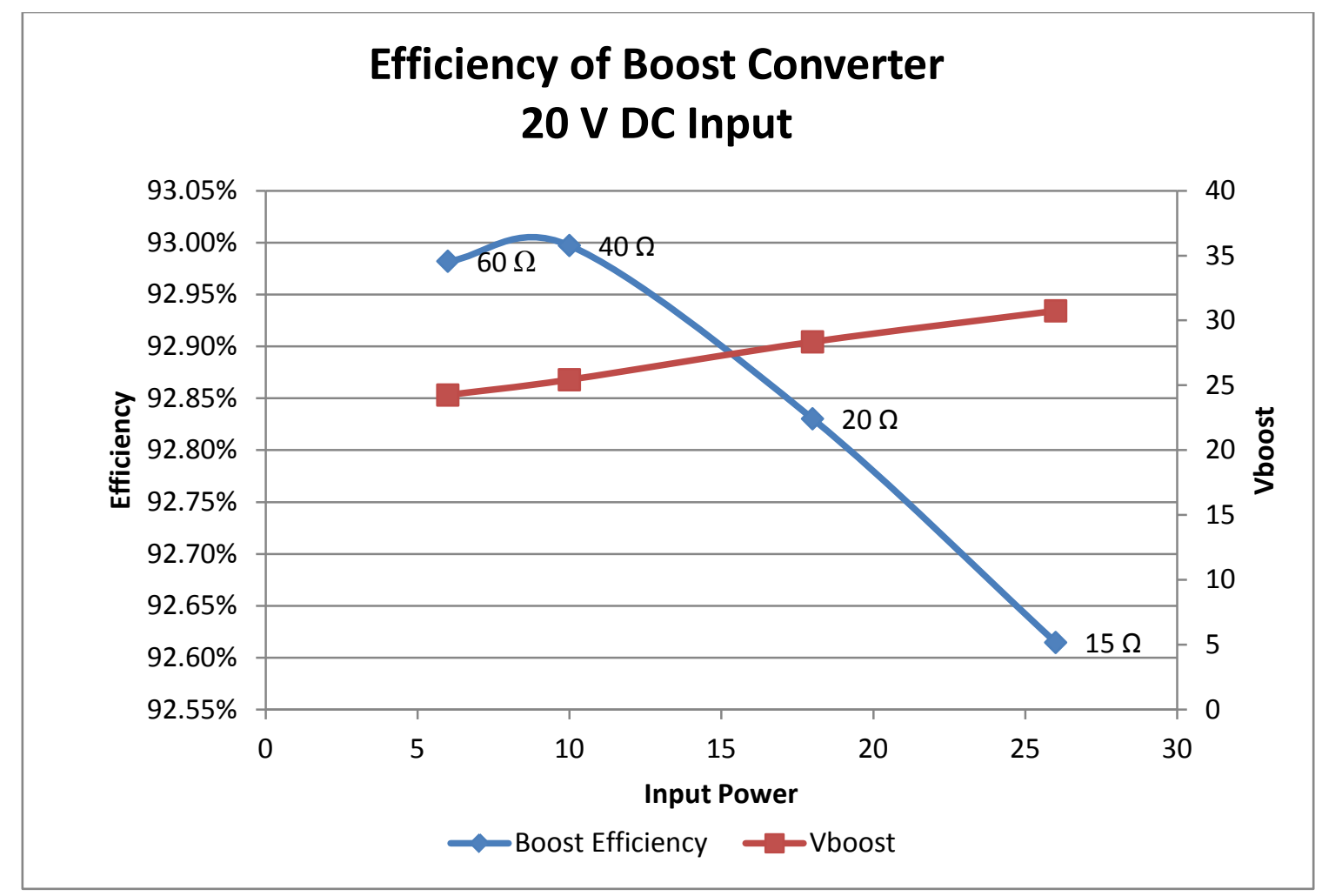

\section{Figure 3-24 - Boost Converter 20 Volt DC Efficiency}

The approximate input power level is labeled to compare the efficiency of the Boost converter with the Buck converter and the total system efficiency in the later sections. It should be mentioned that this efficiency measure is DC efficiency and represents only a single point along the input voltage curve when the input voltage is 20 Volts with the output voltage controlled by the Buck MEECC. A total system efficiency with an AC input voltage waveform will be discussed in Section 5.4.

\subsection{Conclusion}

A solution to the design requirement of the Power Electronic Module to maximize the amount of power extracted from the limited power that is generated by the human powered generator by controlling the input current was presented in this chapter. Three different control methods all using different variations of emulating a constant input resistor value based on user specified values were presented to meet the requirements of the Power Electronics Module. A digitally controlled 
prototype was designed to test and validate the control theories and the circuit was populated on a Printed Circuit Board. The small signal transfer function of the Boost converter was derived from duty cycle to inductor current in the s-domain and then transformed into the z-domain including the digitization effects. The DC gain of the transfer function was used to determine that a Voltage Adaptive Gain controller can help to optimize the compensator of the converter by normalizing the DC gain of the transfer function and increased the low output voltage bandwidth from $1.06 \mathrm{kHz}$ to $11.8 \mathrm{kHz}$ with a phase margin of 79.3 degrees. This resulted in a decrease in current rise time of $660 \mu$ s at low output voltage condition from $13.2 \mathrm{~ms}$. Experimental results were captured with oscilloscope waveforms to validate the operation of the three different control modes, Constant, Variable, and Dynamic $\mathrm{R}_{\text {in }}$ control modes. The waveforms verified the proper operation of the different control modes with the experimental waveforms matching the predicted operation waveforms. The DC efficiency of the Variable $\mathrm{R}_{\text {in }}$ Controlled Boost converter was measured for an input voltage of $20 \mathrm{~V}$ DC at four different emulated resistor values with the peak efficiency measured at $93 \%$ for an emulated resistance of $40 \Omega$ and an input power of 10 Watts. 


\section{Chapter 4}

\section{Buck Control Strategy}

\subsection{Introduction}

This chapter will describe the control methods and controllers that are used to control the Buck converter and how they help the PEM achieve the design requirements that were outlined in Chapter 2. The design requirements of the Buck converter up to this point are for the Buck converter to minimize the amount of energy stored in the ESC by regulating the voltage of the ESC, $\mathrm{V}_{\text {boost }}$, to its minimum value. As well as regulating the charge of the battery pack to charge as fast as possible while staying inside the charging limitations of the battery, namely the current must not exceed the 1C charge rate and the voltage must not exceed 4.2 Volts/cell. This chapter will describe the controllers that make up the Buck Maximum Energy Extraction and Charge Control scheme. The requirements and design of the current loop compensator and voltage loop compensator will be covered as well as the Voltage Adaptive Gain compensator will be designed for the Buck current loop. The MEECC controller is tested to ensure that the Buck converter meets the design requirements of the PEM. The next section will describe the controllers that make up the Buck Maximum Energy Extraction and Charge Control Scheme.

\subsection{Buck Maximum Energy Extraction and Charge Control Scheme}

This section will describe the Maximum Energy Extraction and Charge Controller and how this controller helps the PEM achieve the remaining design requirements. The Maximum Energy Extraction and Charge Controller, or MEECC, is responsible for two of the design requirements of the PEM. The first and most important requirement of the MEECC is the charge regulation of the Li-Po battery pack, which includes the limiting of the maximum charging current to the $1 \mathrm{C}$ charge 
rate as well as limiting the maximum charging voltage of the battery to 4.2 Volts/cell as given by equations (4.1) and (4.2) .

$$
\begin{gathered}
I_{\text {ССMAX }}=1 \mathrm{C}=2 \mathrm{~A} \\
V_{\text {CVMAX }}=4.2 \mathrm{~V} / \mathrm{Ce} l \mathrm{ll}=8.4 \mathrm{~V}
\end{gathered}
$$

While regulating the charging conditions of the battery back, the MEECC is also required to maximize the amount of energy that is transferred from the ESC into the battery. The MEECC does this by controlling the discharge current of the ESC, or Buck charging current to minimize the voltage in the ESC to its minimum value.

A high level power flow diagram has been provided in Figure 4-1 to help the reader understand the power flow through the PEM with the addition of the MEECC Controller. The voltage of the generator is $V_{\text {in }}$ and is based on the user and the walking speed of the user. The voltage of the Energy Storage Capacitor is $\mathrm{V}_{\text {Boost }}$ and the change of $\mathrm{V}_{\text {Boost }}$ is determined by the input and output power level of the PEM. The voltage of the battery pack is $\mathrm{V}_{\text {batt }}$ and is determined by the state of charge and charging condition of the battery. The input power of the PEM is controlled by the value of $\mathrm{V}_{\text {in }}$ and $\mathrm{R}_{\mathrm{in}}$, and is limited by the user's effort level. The output power of the PEM is controlled by the value of $\mathrm{V}_{\text {Boost }}$ and is limited by the maximum battery charging power and the minimum voltage requirement of $V_{\text {Boost }}$. The output power is varied to regulate the voltage $V_{\text {Boost }}$ by regulating the charging current of the battery. 


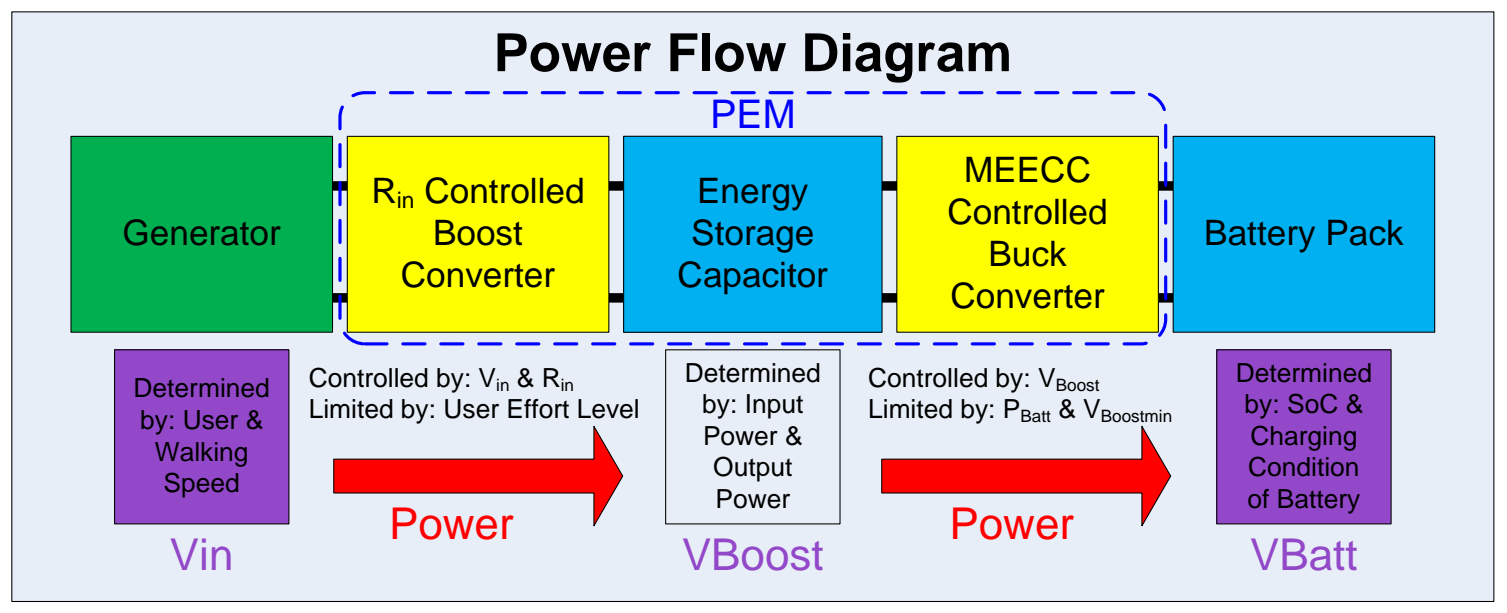

Figure 4-1 - High Level Power Flow Diagram

The charging regulation of the battery pack and the regulation of the ESC voltage $\mathrm{V}_{\text {Boost }}$ are handled by the combination of the four controllers that make up the MEECC and are shown in Figure 4-2.

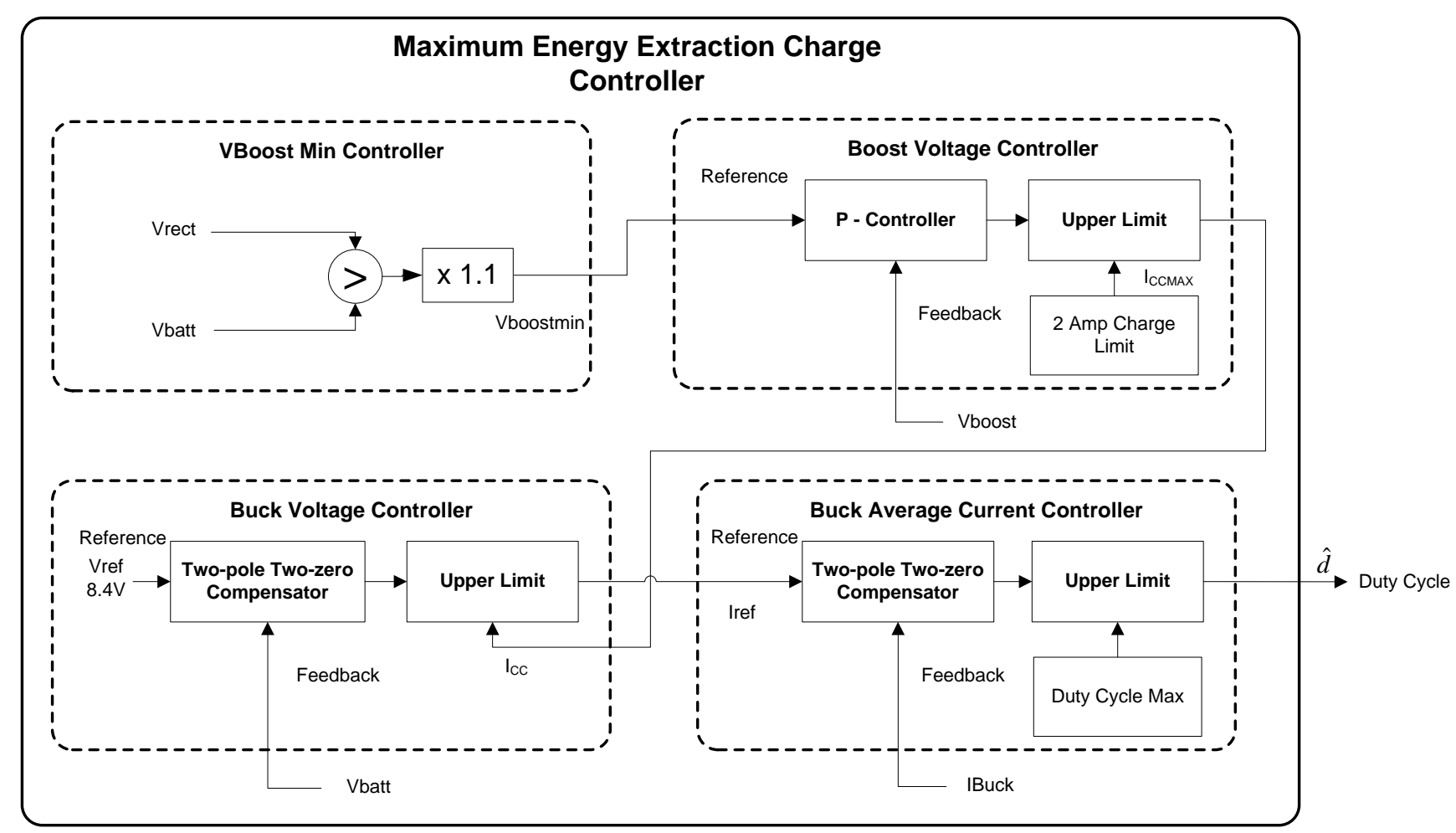

Figure 4-2 - Maximum Energy Extraction Charge Controller Block Diagram 
The charging regulation of the lithium polymer battery pack requires two different modes of charging as explained in Section 2.3, the CC charging mode is used to limit the maximum charging current of the battery pack and the CV charging mode is used to limit the maximum charging voltage of the battery. These two modes are controlled using two controllers to create a two loop controller with a current loop to control the charging current and a voltage loop to control the charging voltage as can be observed in Figure 4-3.

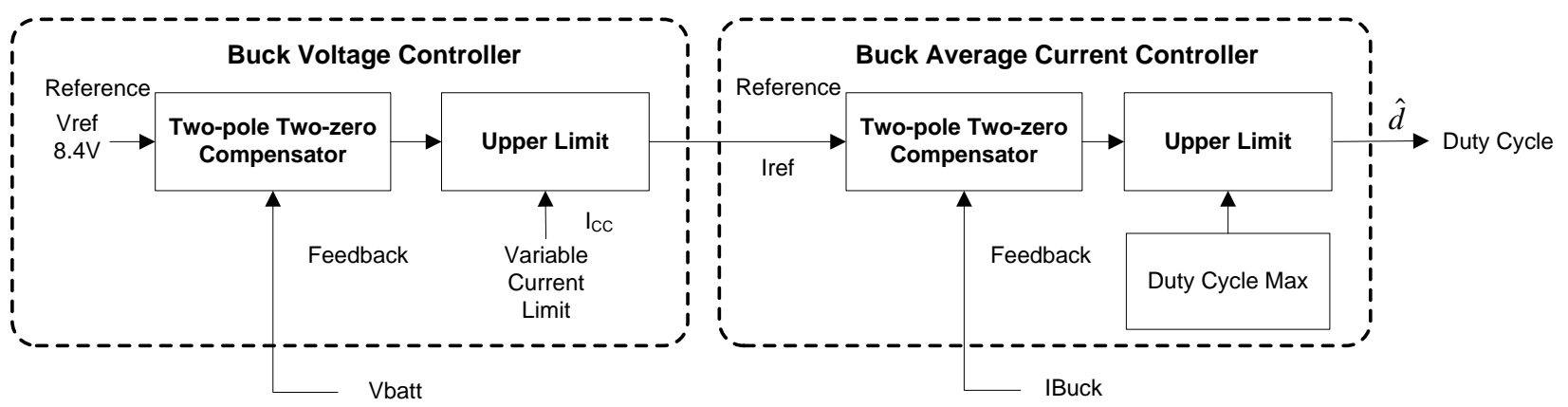

Figure 4-3 - Two Loop Controller for Control of Charging Current and Charging Voltage

The current loop is realized by the Buck Average Current Controller and utilizes a conventional average current mode control scheme consisting of a two-pole two-zero compensator and duty cycle maximum limiter. The feedback of the average current controller is derived from the sensed battery charging current, which is sensed by a current sense resistor and current sense amplifier and sampled by the on board ADC. The reference current for the average current controller is created by the voltage loop which is the Buck Voltage Controller, as observed in Figure 4-3. The operation of the average current controller is governed by equation (4.3), where $I_{\text {ref }}$ is the reference current, $I_{\text {Buck }}$ is the feedback signal, $G_{\text {ciBuck }}(\mathrm{z})$ is the transfer function of the two-pole two-zero average current compensator and $\hat{d}$ is the output duty cycle for the Buck converter. The duty cycle is limited by $\mathrm{D}_{\max }$ which is the maximum duty cycle. The maximum duty cycle limitation is determined by the MOSFET driver of the Buck converter. 


$$
\left(I_{\text {ref }}-I_{\text {Buck }}\right) \cdot G_{\text {ciBuck }}(z)=\hat{d} \text {, where } 0 \leq \hat{d} \leq D_{\max }
$$

The Buck Voltage Controller is similar to a typical voltage controller. However, it has one main difference; the Buck Voltage Controller has an upper limit set point for the maximum reference current that it can pass on to the Average Current Controller. In a typical lithium battery charge controller the upper limit would be set to the maximum charging current rate of 1C. However, with the Buck Voltage Controller the upper limit is a variable that is derived from the Boost Voltage Controller which is the third controller of the MEECC. The Boost Voltage Controller is tasked with maximizing the energy transfer from the ESC to the batteries by minimizing the voltage of the ESC. The reference value of the Buck Voltage Controller $V_{\text {ref }}$ is set at $V_{C V m a x}$ as given by equation (4.2) and (4.4).

$$
V_{\text {ref }}=V_{\text {CVMAX }}
$$

The feedback signal is the battery voltage divided down using a voltage divider and sampled by the ADC. The operation of the Buck Voltage Controller is governed by equation (4.5), where $V_{\text {ref }}$ is the reference voltage, $V_{\text {batt }}$ is the feedback signal, $G_{\text {cvBuck }}(z)$ is the transfer function of the two-pole two-zero voltage compensator and $\mathrm{I}_{\text {ref }}$ is the output of the voltage controller which is the reference current for the average current controller. The output $\mathrm{I}_{\mathrm{ref}}$ is limited by $\mathrm{I}_{\mathrm{CC}}$, the output of the Boost Voltage Controller.

$$
\left(V_{\text {ref }}-V_{\text {batt }}\right) \cdot G_{c v B u c k}(z)=I_{\text {ref }} \text {, where } 0 \leq I_{\text {ref }} \leq I_{C C}
$$

The Buck Voltage Controller and Buck Average Current Controller work together to limit the charging current and regulate the charging voltage of the battery as a typical lithium based charger would with the exception that the CC charging rate is limited by the Boost Voltage Controller. 
The Boost Voltage Controller will provide the Buck Voltage Controller with the upper bound for the CC charging rate. The Boost Voltage Controller will base this upper limit on how much energy is stored in the ESC that is available to be transferred to the battery which is represented by a $\mathrm{V}_{\text {boost }}$ voltage higher than the minimum value $\mathrm{V}_{\text {boostmin. }}$ The Boost Voltage Controller calculates the difference between the reference voltage, $V_{\text {boostmin }}$ and feedback signals, $V_{\text {boost }}$ which is sensed using a voltage divider and sampled by the ADC, the difference is fed into a P-Controller. The output of the P-Controller is limited to the $1 \mathrm{C}$ rate of the battery pack by the upper limit block and provides the upper bound for the reference current command of the Buck Voltage Controller, $\mathrm{I}_{\mathrm{CC}}$ as shown in Figure 4-4.

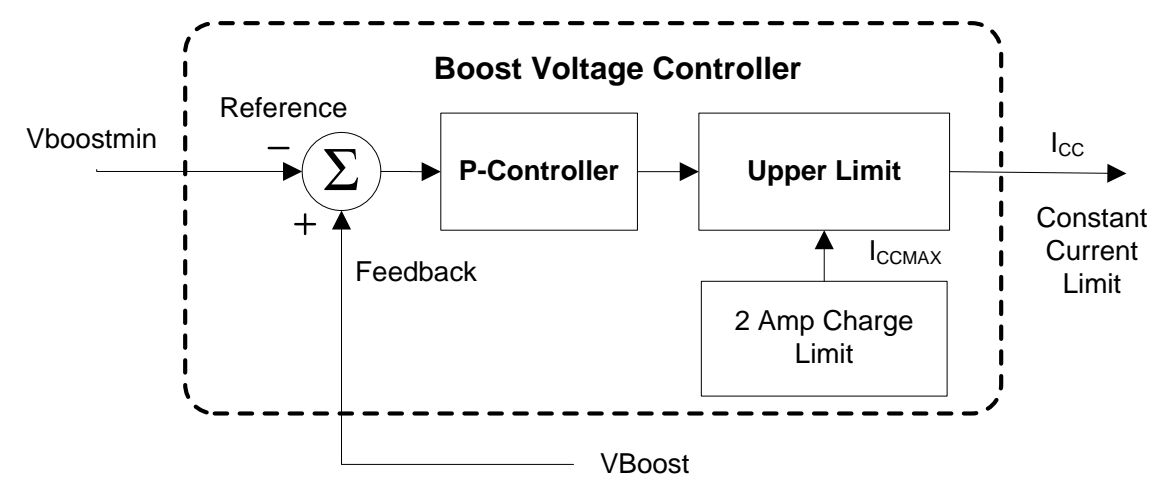

Figure 4-4 - Boost Voltage Controller

The Boost Voltage Controller is governed by equation (4.6), where $V_{\text {Boostmin }}$ is the reference voltage, $\mathrm{V}_{\text {Boost }}$ is the feedback signal, $\mathrm{G}_{\mathrm{P}}$ is the gain of the P-Controller and $\mathrm{I}_{\mathrm{CC}}$ is the output of the Boost Voltage Controller which provides the upper limit for the CC rate of the Buck Voltage Controller. The output $\mathrm{I}_{\mathrm{CC}}$ is limited by $\mathrm{I}_{\mathrm{CCMAx}}$, the maximum current charge rate of the battery pack.

$$
\left(V_{\text {Boost }}-V_{\text {Boost min }}\right) \cdot G_{P}=I_{C C} \text {, where } 0 \leq I_{C C} \leq I_{\text {CCMAX }}
$$


The current command upper limit from the Boost Voltage Controller will dictate how much energy is stored in the ESC, when this signal is above zero there is extra energy in the capacitor that can be transferred to the battery.

A P-Controller is sufficient to control $\mathrm{V}_{\text {Boost }}$, as in the regulation of $\mathrm{V}_{\text {Boost }}$ we do not care if the voltage is slightly higher than the reference level of $\mathrm{V}_{\text {boostmin. }}$. However, $\mathrm{V}_{\text {Boost }}$ must never drop below the $\mathrm{V}_{\text {Boostmin }}$ level as in this case the Boost converter will lose regulation of the input current. Using a P-Controller will ensure that we do not under regulate $\mathrm{V}_{\text {Boost }}$. The steady state error that the P-Controller inherently suffers from can be minimized by using a large gain for the proportional gain block. As long as the value of the gain coefficient is not too large, the controller will not suffer from oscillations caused by instability that a PI-controller could introduce. A PI-controller has memory of the previous value which can cause the regulator to undershoot the $\mathrm{V}_{\text {boostmin }}$ voltage level.

The reference signal for the Boost Voltage Controller, $V_{\text {Boostmin, }}$ is a signal derived from the VBoost Min Controller. The VBoost Min Controller determines the minimum level of voltage required in the ESC for the proper regulation of the input current in the Boost converter as well as ensuring the proper operation of the Buck converter. The controller's output is the maximum of two signals, $\mathrm{V}_{\text {rect }}$ and $\mathrm{V}_{\text {batt }}$, both sensed by the ADC, multiplied by 1.1 to provide the reference voltage for the Boost Voltage Controller, as shown in Figure 4-5. The extra $10 \%$ is necessary due to duty cycle limitations of the driving circuits as well as for system stability. 


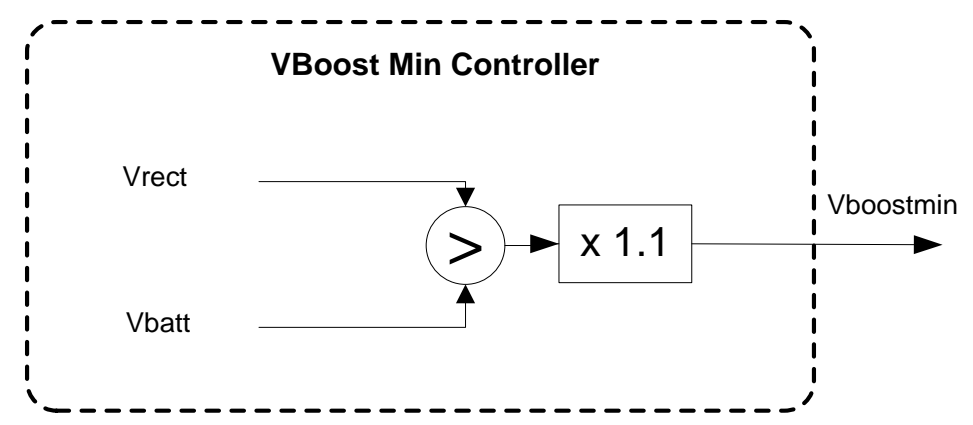

\section{Figure 4-5 - VBoost Min Controller}

The equation governing the operation of the VBoost Min Controller is given in equation (4.7),

where $V_{\text {rect }}$ is the rectified input voltage, $V_{\text {batt }}$ is the battery voltage and $V_{\text {Boostmin }}$ in the minimum voltage level of $\mathrm{V}_{\text {Boost }}$ to allow proper regulation of the input current and charging current of the battery.

$$
\begin{aligned}
& V_{\text {Boost min }}=V_{\text {rect }} \cdot 1.1 \text {, when } V_{\text {rect }} \geq V_{\text {batt }} \\
& V_{\text {Boost min }}=V_{\text {batt }} \cdot 1.1 \text {, when } V_{\text {batt }}>V_{\text {rect }}
\end{aligned}
$$

These four controllers create the MEECC and together will minimize the voltage stored in the ESC, reducing the energy stored in the ESC leaving more room for future energy storage as well as maximizing the amount of energy transferred into the batteries all while keeping the battery pack within the charging limitations.

\subsection{Digitally Controlled Buck Converter Plant Transfer Function}

This section will cover the derivation of the small signal transfer function of the Buck converter in the s-domain for both the average current mode control loop as well as the voltage control loop. The transfer functions will be discretized using zero-order-hold including the effects of sample and 
hold and computational delay. A suitable compensator for the current loop and voltage loop will be selected once the discretized transfer functions have been found.

The small signal of the Buck converter operating in CCM, shown in Figure 4-6, can be determined by averaging the converter state equations.

\section{Buck Converter Operating in $\mathrm{CCM}$}

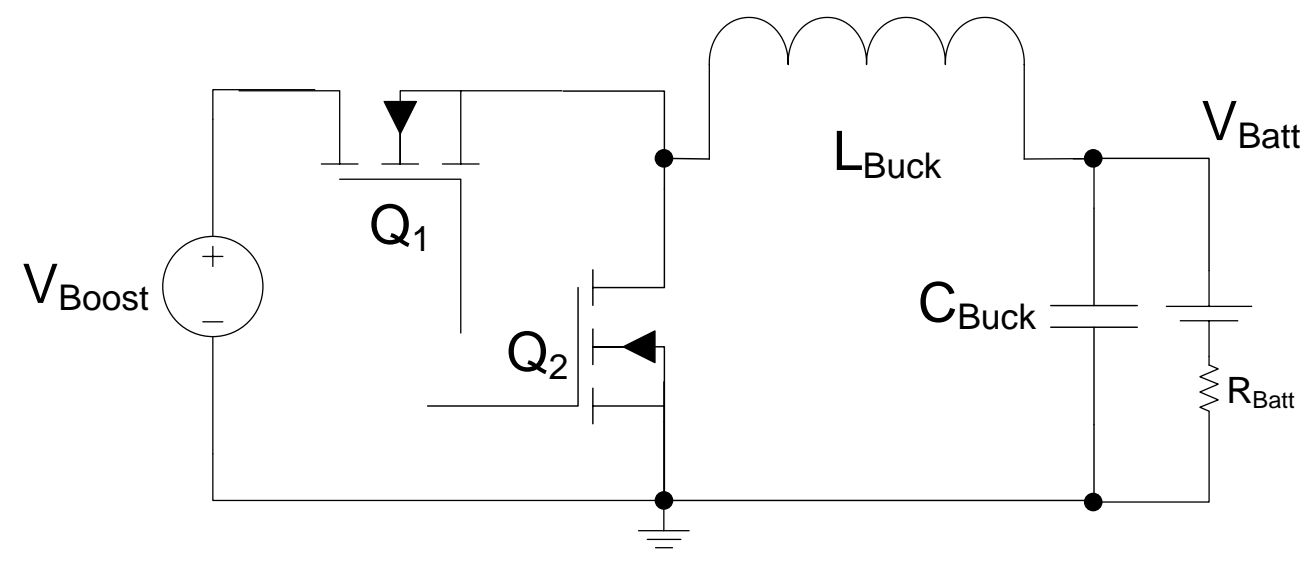

Figure 4-6 - Schematic of Buck Converter Operating in CCM

The averaged equations for the Buck Converter are given in (4.8) and (4.9)

$$
\begin{aligned}
\frac{d i_{L}}{d t} & =\frac{D\left(V_{\text {Boost }}\right)-V_{\text {batt }}}{L_{\text {Buck }}} \\
\frac{d V_{\text {batt }}}{d t} & =\frac{i_{L}}{C_{\text {Buck }}}-\frac{V_{\text {batt }}}{R_{\text {Batt }} C_{\text {Buck }}}
\end{aligned}
$$

The control parameters, input and output variables are all replaced with a steady-state DC value and a small time-varying component shown in (4.10) and (4.11).

$$
\frac{d\left(I_{L}+\hat{i}_{L}\right)}{d t}=\frac{(D+\hat{d})\left(V_{\text {Boost }}+\hat{v}_{\text {Boost }}\right)-\left(V_{\text {batt }}+\hat{v}_{\text {batt }}\right)}{L_{\text {Buck }}}
$$




$$
\frac{d\left(V_{\text {batt }}+\hat{v}_{\text {batt }}\right)}{d t}=\frac{\left(I_{L}+\hat{i}_{L}\right)}{C_{\text {Buck }}}-\frac{\left(V_{\text {batt }}+\hat{v}_{\text {batt }}\right)}{R_{\text {Batt }} C_{\text {Buck }}}
$$

After expanding the terms and neglecting the small-signal products and DC products, the equations (4.10) and (4.11) can be rewritten in terms of the small-signals, shown in (4.12) and (4.13)

$$
\begin{aligned}
& \frac{d \hat{i}_{L}}{d t}=\frac{D \hat{v}_{\text {Boost }}+\hat{d} V_{\text {Boost }}-\hat{v}_{\text {batt }}}{L_{\text {Buck }}} \\
& \frac{d \hat{v}_{\text {batt }}}{d t}=\frac{\hat{i}_{L}}{C_{\text {Buck }}}-\frac{\hat{v}_{\text {batt }}}{R_{\text {Batt }} C_{\text {Buck }}}
\end{aligned}
$$

The linearized block diagram of the Buck converter can be created using (4.12) and (4.13) and simplified using block diagram simplification techniques, [31], as illustrated in Figure 4-7 

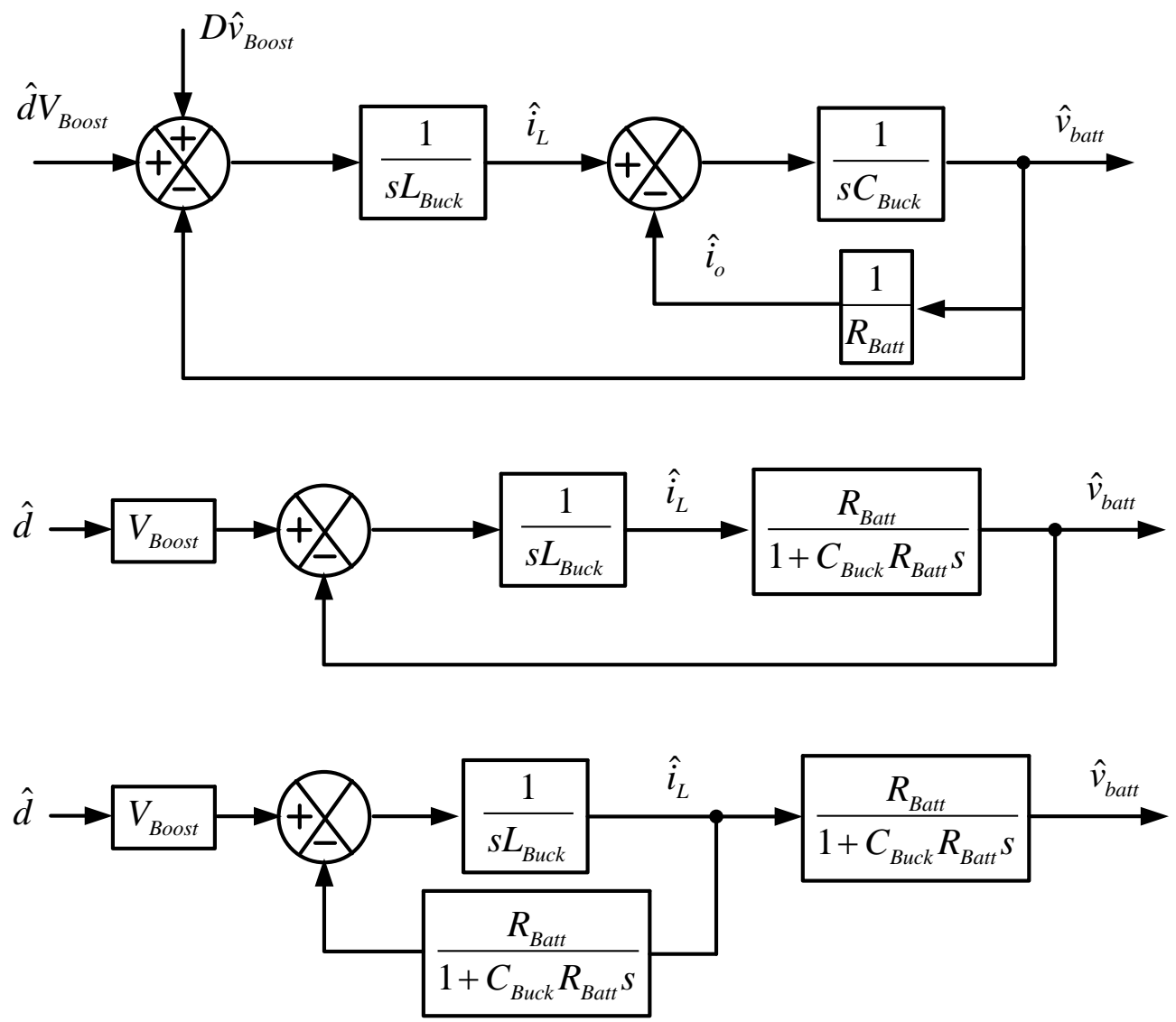

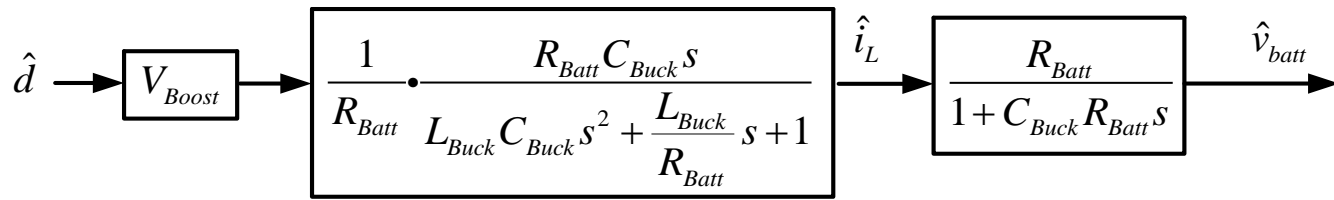

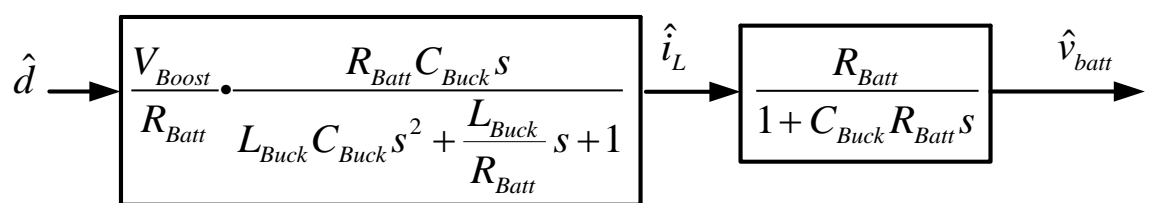

Figure 4-7 - Simplification of Linearized Block Diagram of Buck Converter Operating in CCM 
From the block diagram simplification, the small signal transfer function from duty cycle to inductor current, $\mathrm{G}_{\mathrm{iBuck}}(\mathrm{s})$, is given in the s-domain by equation (4.14). The small signal transfer function from inductor current to output voltage, $G_{v B u c k}(s)$, is given in the s-domain by equation

$$
\begin{gathered}
G_{\text {iBuck }}(s)=\frac{\hat{i}_{L}}{\hat{d}}=\frac{V_{\text {Boost }}}{R_{\text {Batt }}} \cdot \frac{R_{\text {Batt }} C_{\text {Buck }} s}{L_{\text {Buck }} C_{\text {Buck }} s^{2}+\frac{L_{\text {Buck }}}{R_{L}} s+1} \\
G_{v \text { Buck }}(s)=\frac{\hat{v}_{\text {batt }}}{\hat{i}_{L}}=\frac{R_{\text {Batt }}}{R_{\text {Batt }} C_{\text {Buck }} s+1}
\end{gathered}
$$

From the transfer functions, the continuous-time domain closed loop block diagram for the voltage loop and current loop can be determined and is shown in Figure 4-8.

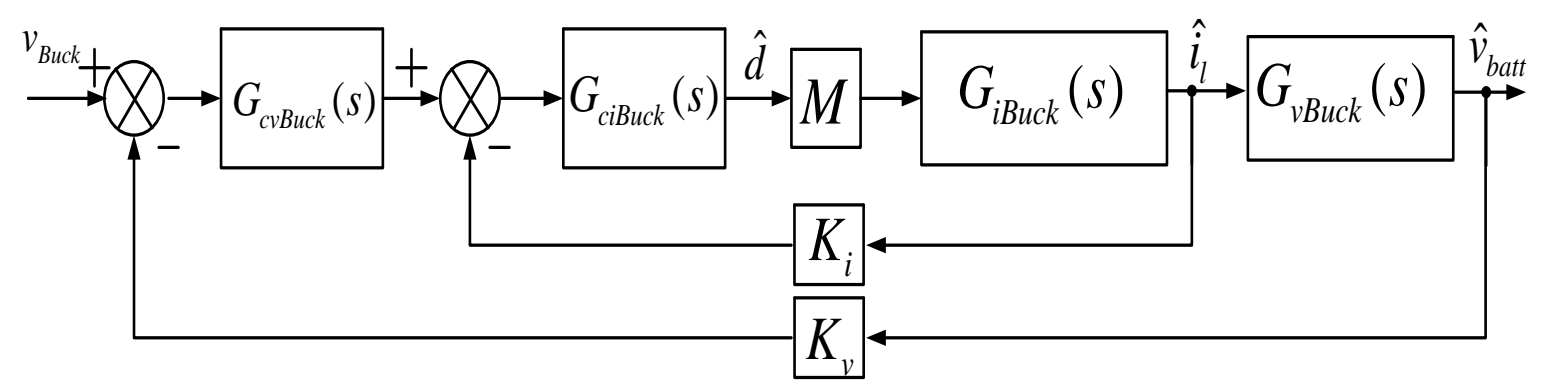

Figure 4-8 - Continuous-Time Domain Closed Loop Buck Voltage and Current Loop

Shown in the figure, $G_{c v B u c k}(s)$ is the transfer function of the voltage loop compensator, $G_{\text {ciBuck }}(s)$ is the transfer function of the current loop compensator, $\mathrm{M}$ is the modulator gain, $\mathrm{K}_{\mathrm{i}}$ is the gain of the current sensing network and $\mathrm{K}_{\mathrm{v}}$ is the gain of the voltage sensing network.

The continuous time transfer function can be discretized with $\mathrm{ZOH}$ to take into account the sample and hold delay as well as the other delays and gains that make up the digital system as was done 
with the Boost converter. Once discretized, the current loop compensator and voltage loop compensator can be directly designed in the digital domain using Matlab’s siso tool [28].

The order of the Buck average current loop transfer function was similar to that of the Boost converter with the addition of the digitization effects, having three poles and two zeros. The compensator was therefore selected to be a digital two-pole two-zero compensator to compensate the Buck average current control loop. The order of the Buck voltage control loop transfer function after the digitization effects were added was a two-pole one-zero system. A PI-compensator would be sufficient to compensate the voltage loop. However, a two-pole two-zero digital compensator was used due to the simplicity of using the same type of controllers throughout the design. The digital two-pole two-zero compensator is the same as that which was derived in Section 3.6, given by equation (3.22) and the discretized equation (3.23). The compensators designed in the digital domain can be directly entered into to the digital controller by using equation (3.23).

\subsection{Buck Two-Pole Two-Zero Compensator with Voltage Adaptive Gain}

The output voltage of the Boost converter which is also the input voltage to the Buck converter, $\mathrm{V}_{\text {boost }}$, is loosely regulated by the Buck Maximum Energy Extraction and Charge Controller. However, the Buck converter cannot always keep $\mathrm{V}_{\text {boost }}$ constant, because of the difference between the input power and output power of the PEM there is the requirement to store energy in the ESC. As it is not always possible for the MEECC to keep $V_{\text {boost }}$ constant the Buck converter's input voltage swing is large and can range from 6 - 80 volts. This has a similar effect to the compensator of the current loop in the Buck converter as the compensator of the current loop in the Boost converter. It is difficult to optimize the compensator for the wide input voltage swing and a compromise between bandwidth and stability must be found when a conventional two-pole two- 
zero compensator is used. Without a properly optimized compensator the response will suffer from low bandwidth at low input voltage and reduced phase margin and stability at high input voltage. A conventional two-pole two-zero compensator could only provide a sub optimal response when used with the Buck converter with the wide input voltage range.

The Voltage Adaptive Compensator Gain that was used to solve this issue caused by the wide output voltage swing in the Boost converter can also be applied to solve this issue caused by a wide input voltage swing in the Buck converter. A similar Voltage Adaptive Compensator Gain can normalize the DC gain of the Buck current loop transfer function for the wide input voltage range. The small-signal transfer function of the Buck average current loop is needed to properly counteract the effect of the variance of the DC gain with the input voltage to the Buck, $\mathrm{V}_{\text {boost }}$.

The small-signal transfer function of the Buck current loop from duty cycle $\hat{d}$ to inductor current $\hat{i}_{L}$ was derived in Section 4.3 as equation (4.14). The DC gain of the transfer function can be derived from equation (4.14) by setting $s=0$ as shown in equation (4.16)

$$
\text { DC Gain }=\frac{V_{\text {boost }}}{R_{\text {batt }}}
$$

From equation (4.16) it can be observed that the DC gain of the Buck average current loop varies with the input voltage to the Buck, $\mathrm{V}_{\text {boost }}$. As the internal resistance of the battery $\mathrm{R}_{\text {batt }}$ is constant over the whole SoC of the battery, the Voltage Adaptive Compensator Gain can be used to cancel the effect of the changing DC gain with the varying input voltage.

In order to compare the difference in dynamic loop response with and without Voltage Adaptive Compensator Gain, a conventional two-pole two-zero compensator was designed for the Buck 
average current loop over the wide input voltage range of the converter. With an input voltage of 10 volts the achievable bandwidth was $1.66 \mathrm{kHz}$ with a phase margin of 42.8 degrees. With an input voltage of 80 volts the achievable bandwidth was $11 \mathrm{kHz}$ with a phase margin 75 as shown in Figure 4-9.

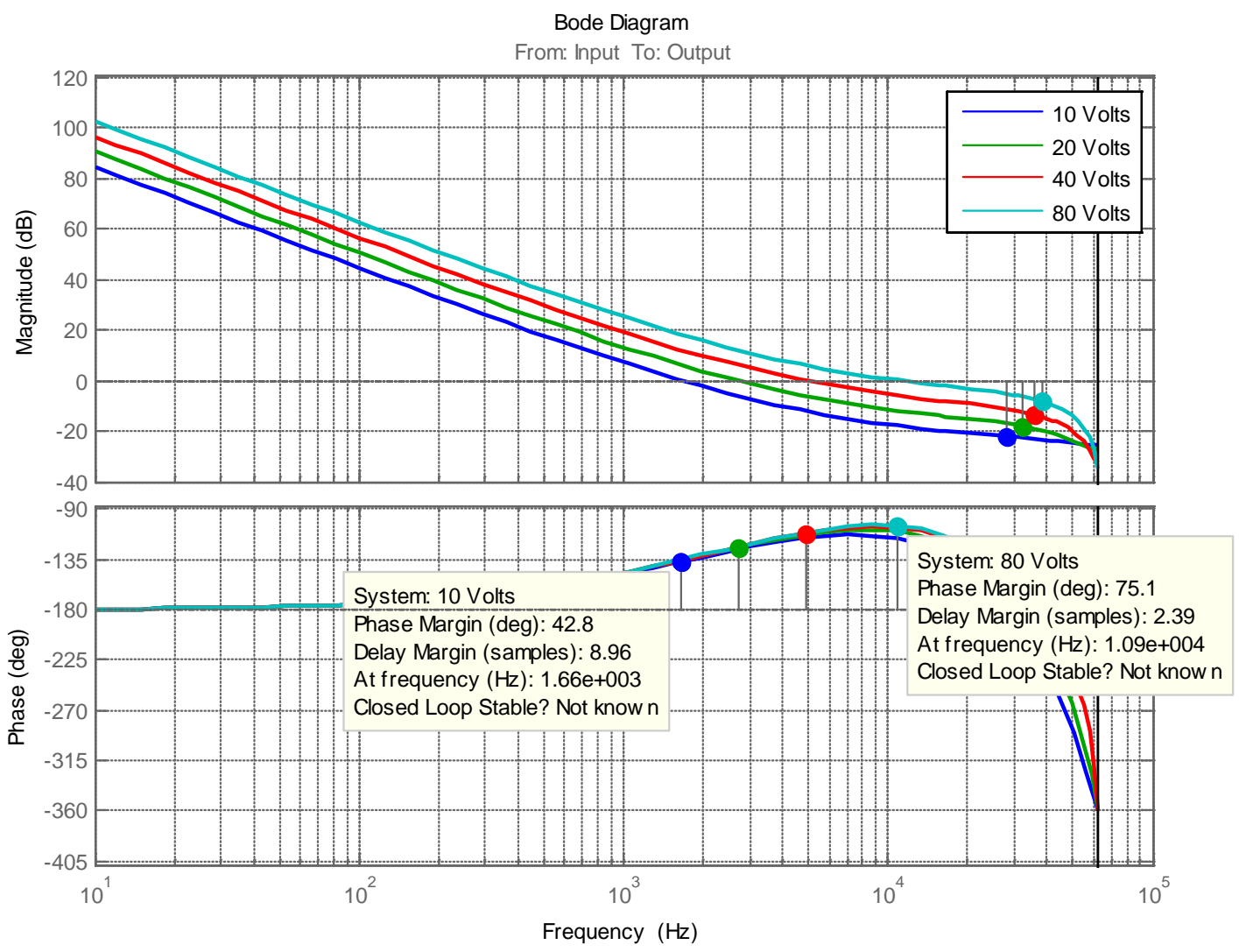

Figure 4-9 - Open Loop Bode Plot of Buck Converter at Different Input Voltages with a Conventional Two-pole Two-zero Compensator

The low bandwidth at the low input voltage condition will cause the rise time for the Buck current to be slow. When the Buck converter is required to turn on the Buck current must step from 0 A to the controlled value. A delay in the turn on time will cause a delay in the transfer of power from the ESC to the battery. 
The MEECC controlled Buck converter using a conventional two-pole two-zero compensator was tested with a step input voltage, $\mathrm{V}_{\text {Boost }}=7-10 \mathrm{~V}$ with $\mathrm{V}_{\text {batt }}=7.5 \mathrm{~V}$ to determine the turn on time of the converter and is shown in Figure 4-10. As can be observed from the figure, the time required for the inductor current to increase to the controlled level from the start of the Control MOSFET duty cycle is measured as $13 \mathrm{~ms}$.

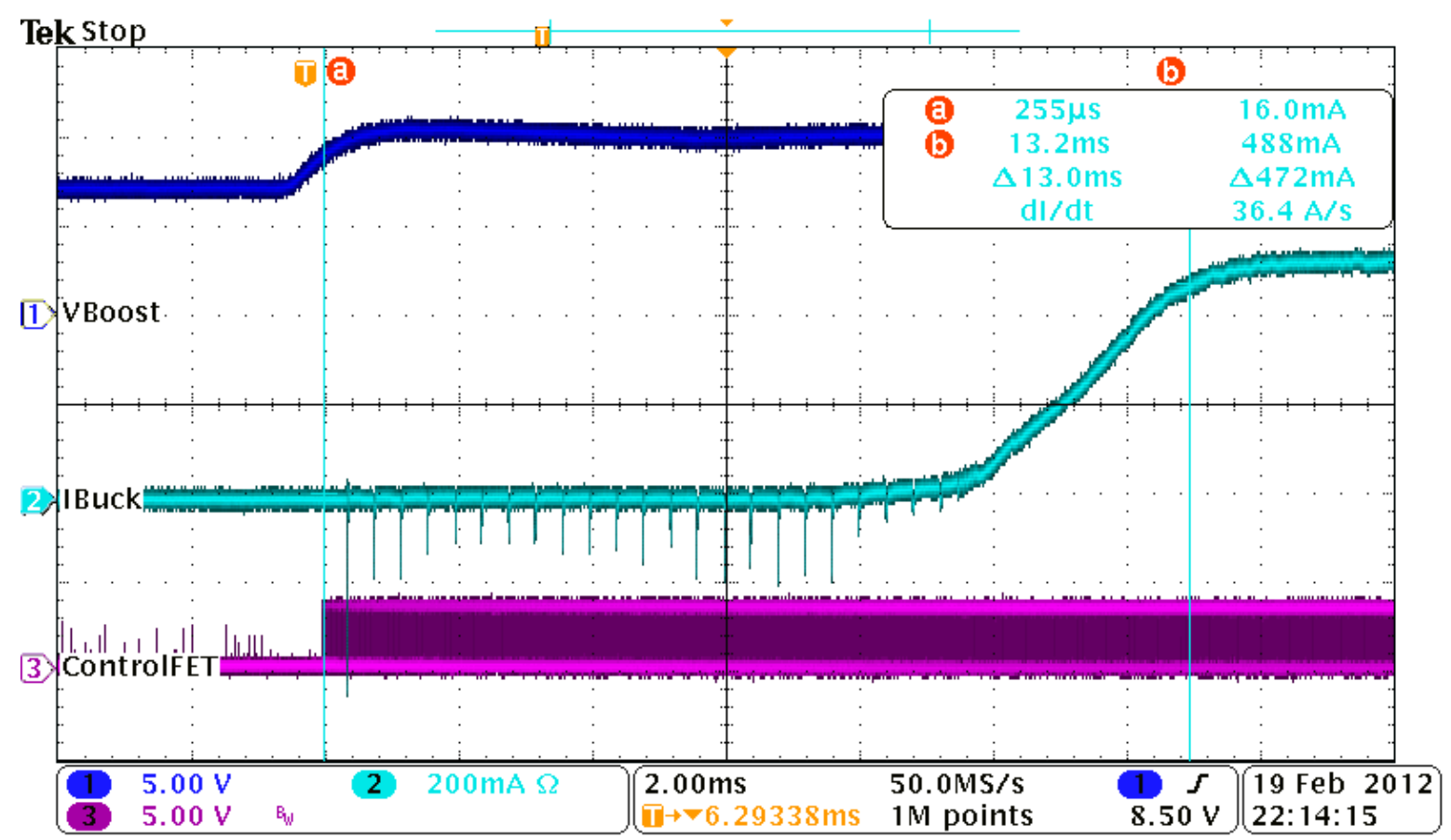

Figure 4-10 - Rise Time of Inductor Current for MEECC Controlled Buck Converter with Conventional Controller

A Voltage Adaptive Gain Compensator is used similar to that explained in Section 3.7 with the equation representing the compensator given by (3.28). The value for the voltage adaptive gain value, $\mathrm{G}_{\text {adp }}$ was described in Table 3-3 as is updated at the same rate as with the Boost converter, at $1 \mathrm{kHz}$. The achievable bandwidth with the Voltage Adaptive Gain Compensator at 10 Volts input 
was increased from $1.6 \mathrm{kHz}$ to $11.3 \mathrm{kHz}$ with a phase margin of 62.4 degrees. The bandwidth and phase margin at an input voltage of 80 volts remains at $11 \mathrm{kHz}$ and 75 degrees of phase margin as shown in Figure 4-11.

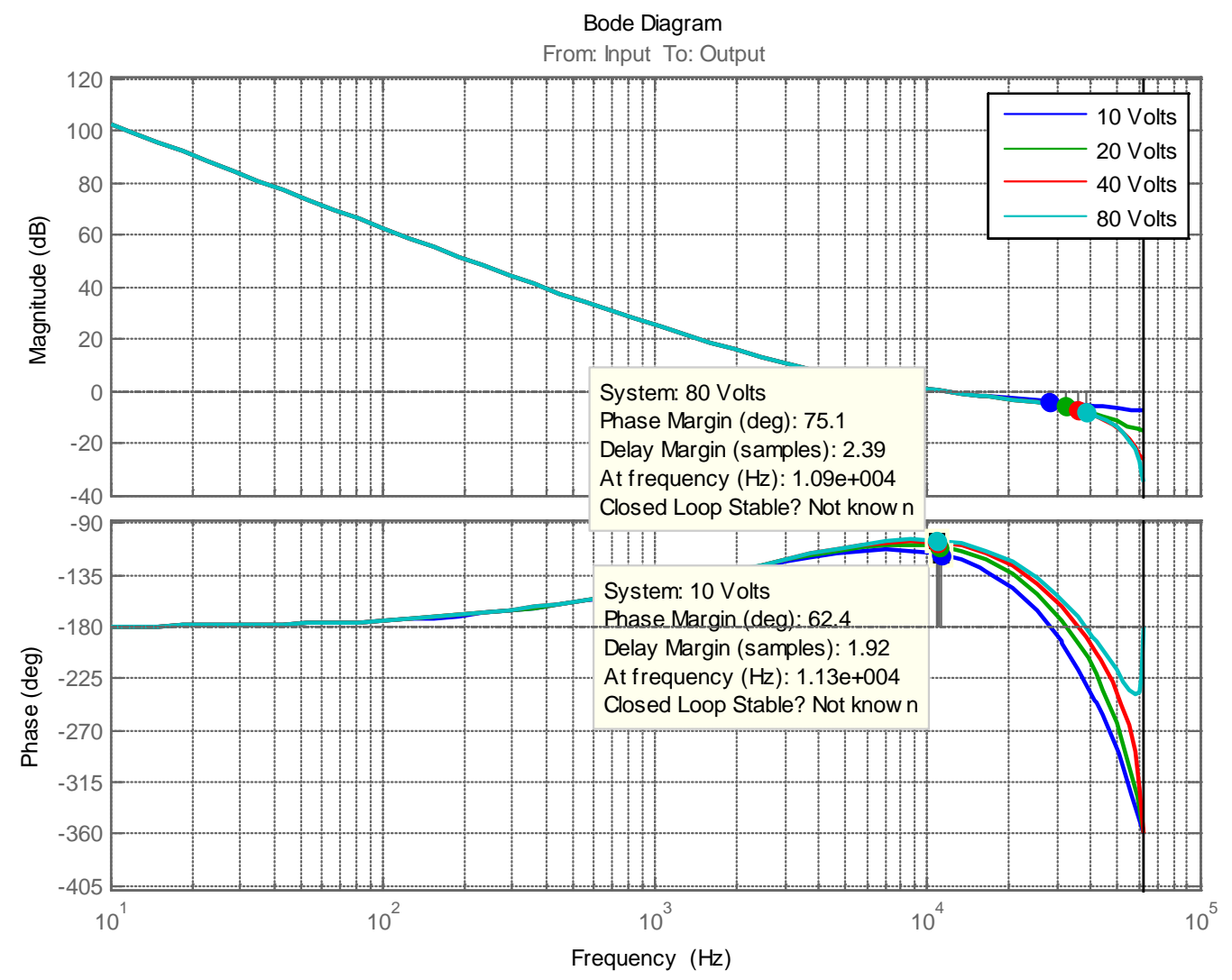

Figure 4-11 - Open Loop Bode Plot of Buck Converter at Different Input Voltages with a Voltage Adaptive Gain Compensator

With the Voltage Adaptive Gain Compensator we can achieve optimal performance over the entire input voltage range of the Buck converter.

The Voltage Adaptive Gain Compensator was tested with a step input voltage, $\mathrm{V}_{\text {Boost }}=7-10 \mathrm{~V}$ with $\mathrm{V}_{\text {battt }}=7.5 \mathrm{~V}$ to determine the improvement of the turn on time of the converter and is shown in 
Figure 4-12. As can be observed from the figure, the time required for the inductor current to increase to the controlled level from the start of the Control MOSFET duty cycle is measured at 3.12 ms. The Voltage Adaptive Gain Compensator has decreased the turn on time of the MEECC controlled Buck converter from 13 ms to 3.12 ms.

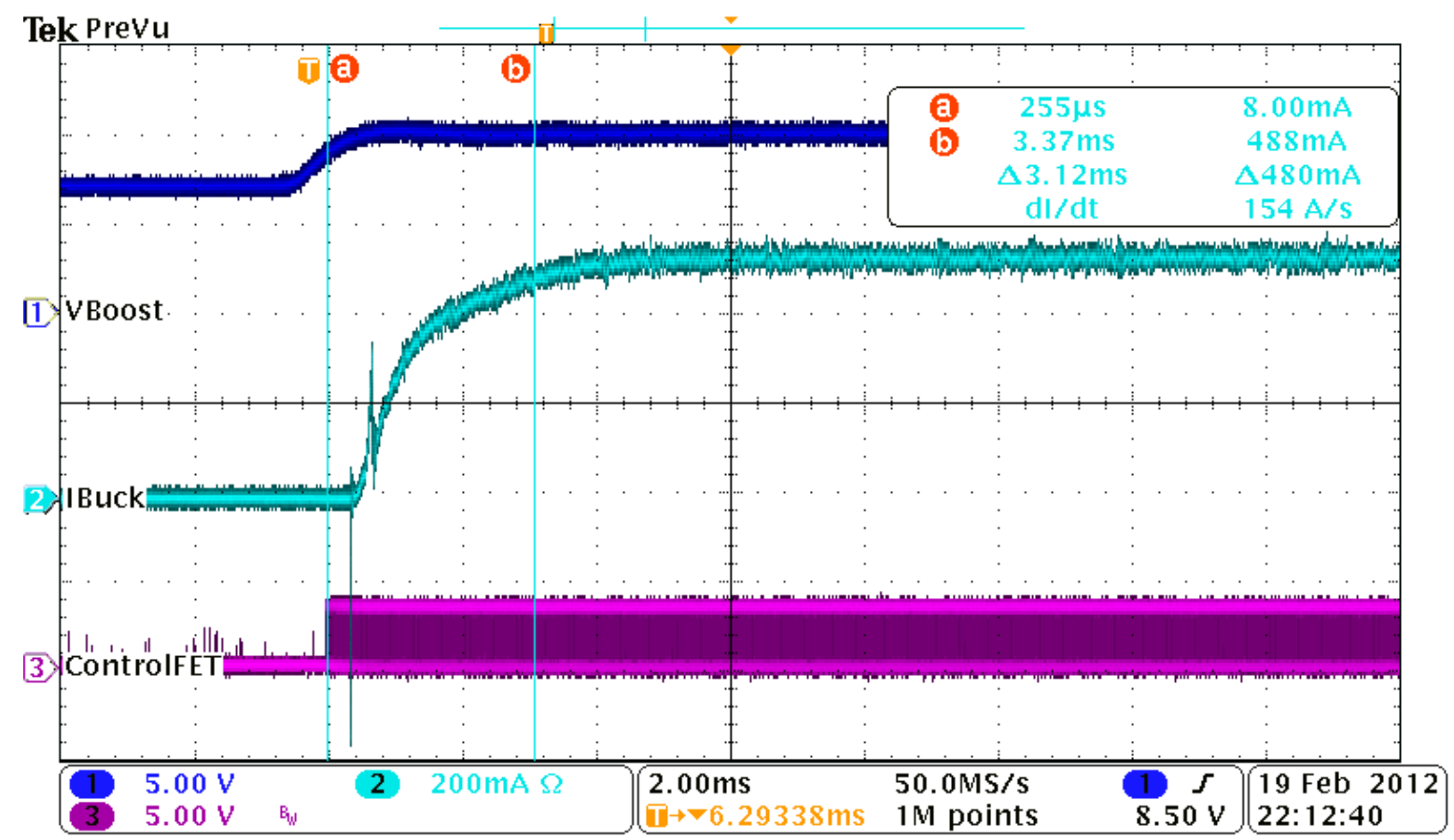

Figure 4-12 - Rise Time of Inductor Current MEECC Controlled Buck Converter with Variable Gain Controller

Similar to the case with the Boost converter a longer delay before the current reaches the desired level would mean a reduction in amount power that is able to be extracted from the generator and stored in the battery pack.

The voltage loop controller design is simplified when using the Voltage Adaptive Gain Compensator for the current loop as the current loop transfer function has been normalized over the input voltage range to the Buck converter. The transfer function from inductor current, $\hat{i}_{l}$ to output 
voltage, $\hat{v}_{o}$ was derived in Section 4.3 and is given by equation (4.15). The DC Gain of the transfer function can be derived by setting $\mathrm{s}=0$ and is given in (4.17)

$$
\text { DCGain }=R_{\text {batt }}
$$

As shown in equation (4.17) the DC transfer function from inductor current, $\hat{i}_{l}$ to output voltage, $\hat{v}_{o}$ is not dependent on $\mathrm{V}_{\text {boost }}$. The transfer function therefore is independent of the input voltage of the Buck converter and a conventional two-pole two-zero compensator can be used. The open loop bode plot of the compensated voltage loop is shown in Figure 4-13. From the figure the bandwidth of the compensator is $500 \mathrm{~Hz}$ with a phase margin of 86.6 degrees.

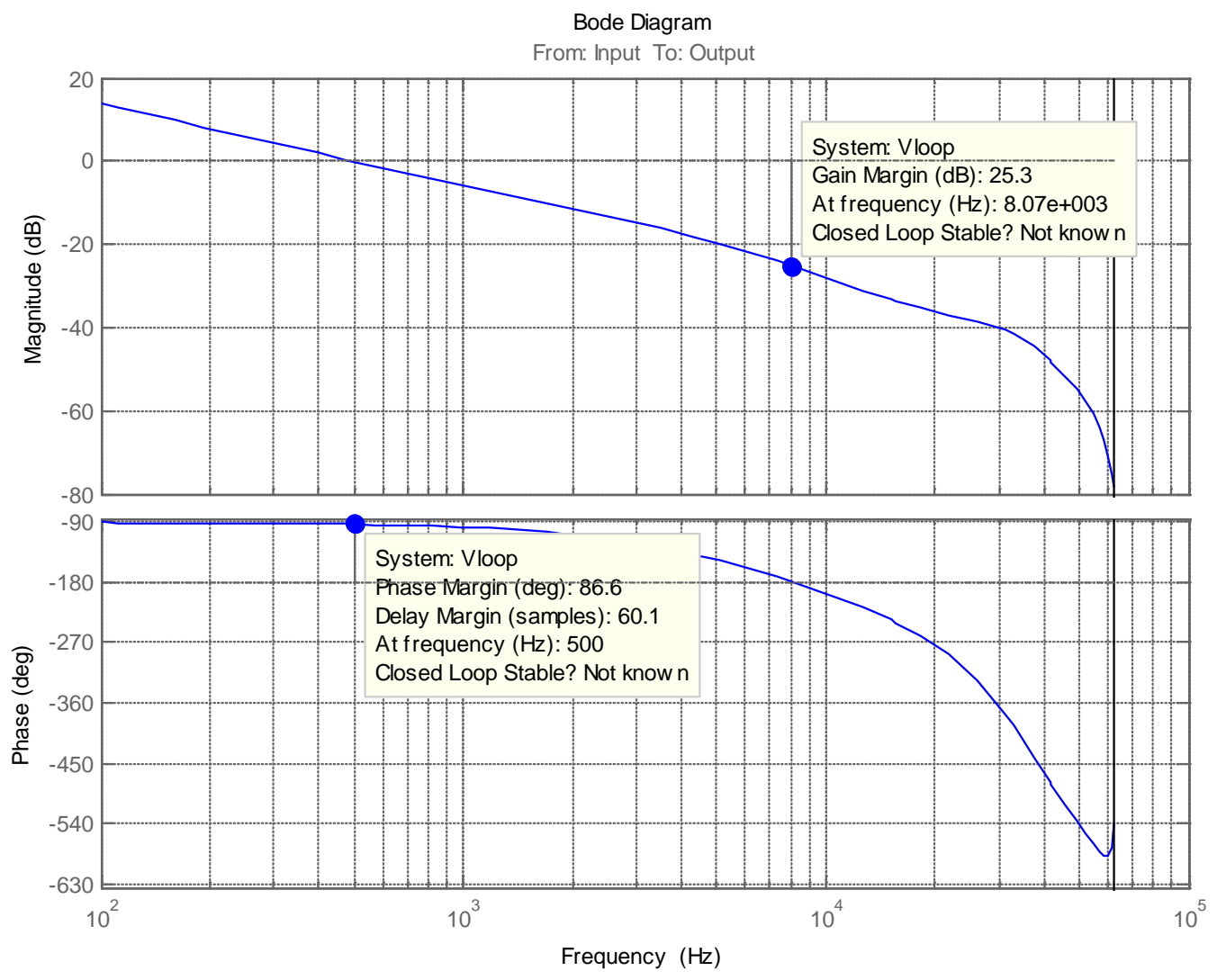

Figure 4-13 - Open Loop Bode Plot of Voltage Loop for Buck Converter 
With a switching frequency of $250 \mathrm{kHz}$ and a sampling frequency of $125 \mathrm{kHz}$ we should be able to achieve a bandwidth of $12.5 \mathrm{kHz}$ for the average current mode control based on the general rule of being able to achieve a bandwidth of around $1 / 10^{\text {th }}$ the sampling frequency [30]. We have designed a slightly lower bandwidth for three main reasons; the first is that we do not need a very fast dynamic response for our converter as the load dynamics are very slow as we are only charging a battery. We are trying to demonstrate the operation of the control and a higher stability margin is desired, so the bandwidth is decreased. The third reason is that we can reduce the computational power required to run the controllers by sampling at half the switching frequency. As there is less power drawn by the DSP more of the harvested energy can be stored in the battery pack. The bandwidth of the voltage loop is typically $1 / 10^{\text {th }}$ of the current loop bandwidth [30], however, we have chosen a lower bandwidth of $500 \mathrm{~Hz}$ again mainly for the increased stability and phase margin a lower bandwidth will provide. As the voltage we are regulating is the battery pack voltage, which has relatively slow dynamics, there is no need for a fast dynamic response voltage controller.

\subsection{Driving of Synchronous Buck Control FET without the use of an Isolated Supply Voltage}

A conventional high-side bootstrapped driver cannot drive the control MOSFET of the Buck converter without the use of an isolated supply voltage because the battery voltage is always present at the output of the Buck converter. The design of the PEM requires that the battery pack, $V_{\text {batt }}$, be always connected to the output of the Buck converter, $V_{0}$, as shown in Figure 4-14, this makes it difficult for the bootstrap capacitor of the driver to charge because the switching node is not clamped to ground through $\mathrm{Q}_{2}$ until there is forward inductor current. The inductor current cannot be forward biased until the control MOSFET, $\mathrm{Q}_{1}$ is turned on and $\mathrm{Q}_{1}$ cannot be turned on until the bootstrap capacitor is charged. Typically an isolated voltage supply for the driver would be 
used to allow the control MOSFET to be turned on without the need for the switching node to be first clamped to ground. This increases the cost and size of the converter as a separate isolated power supply is needed with a transformer to provide the isolation.

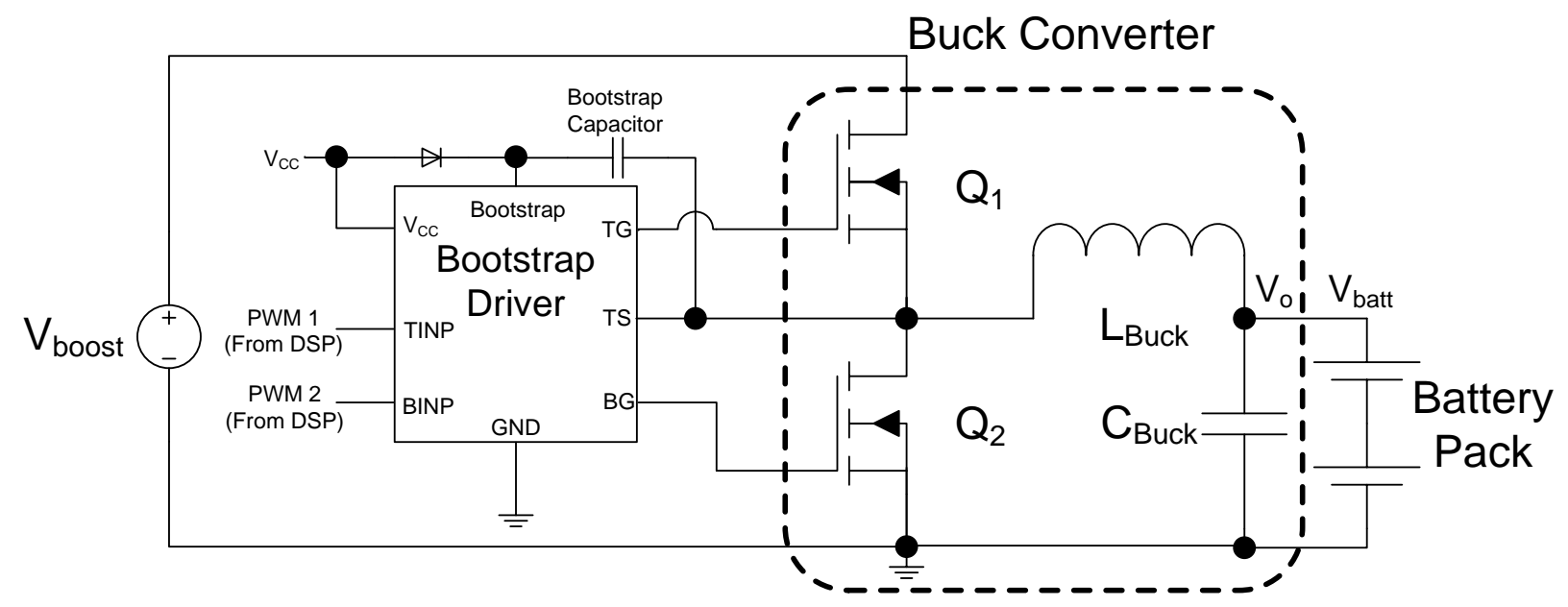

\section{Figure 4-14 - Buck Converter with Bootstrap Driver and Synchronous Switch [32]}

The following technique is proposed to solve this problem, by using some logic and control of the synchronous MOSFET, $\mathrm{Q}_{2}$, to clamp the switching node of the Buck converter to ground for enough time to charge the bootstrap capacitor and avoid the need for an isolated supply. As we are already using a DSP for the digital controllers in the design of the PEM this solution can be realized with no additional cost or size to the converter.

Shown in Figure 4-15 is the control signals from the DSP for the two MOSFETs, ControlFet driving $\mathrm{Q}_{1}$ and SyncFet driving $\mathrm{Q}_{2}$ of Figure 4-14. From Figure 4-15 observing the ControlFet waveform, the DSP is attempting to drive $\mathrm{Q}_{1}$ during the first pulse, however, it can also be observed in the figure by the switching node waveform, VSwitching_Node as well as from the inductor current waveform, I(Lbuck) that $\mathrm{Q}_{1}$ did not turn on. The $\mathrm{Q}_{1}$ MOSEFT could not be turned on 
because the driver bootstrap capacitor was not charged, shown by the waveform Vbootstrap in the figure. The bootstrap capacitor cannot charge until the switching node has been clamped to ground through $\mathrm{Q}_{2}$. The DSP is used to control $\mathrm{Q}_{2}$ to turn on for 5\% duty cycle just after $\mathrm{Q}_{1}$ has turned off causing the switching node to be clamped to ground, shown by the VSwitching_Node waveform. The bootstrap capacitor can charge in this duration when the switching node is clamped to ground and it can be seen charged to 5 Volts above VSwitching_Node once $\mathrm{Q}_{2}$ has turned off from the Vbootstrap waveform. The $\mathrm{Q}_{2}$ MOSFET is turned on for the $5 \%$ duty cycle directly following $\mathrm{Q}_{1}$ turning off to prevent negative inductor current once the inductor current has become positive. This can be observed in Figure 4-15 with the inductor current waveform, I(Lbuck), unaltered by the second pulse from the $\mathrm{Q}_{2}$ MOSFET. The Q2 MOSFET is only turned on for 5\% duty cycle to allow enough time for the bootstrap capacitor to charge and to minimize any negative inductor current. After a few pulses of the $\mathrm{Q}_{2}$ MOSFET the forward inductor current will keep the switching node clamped to ground through the forward diode of $\mathrm{Q}_{2}$ during the complementary of the Q1 MOSFET duty cycle. The 5\% duty cycle pulse of Q2 is no longer needed and the DSP will turn off the control signal to $\mathrm{Q}_{2}$. As long as the inductor current remains forward biased the driver's bootstrap capacitor will remain charged. Once the inductor current has increased above a threshold level the DSP will control the synchronous MOSFET $\mathrm{Q}_{2}$ with the complementary signal of $\mathrm{Q}_{1}$. In the prototype the value of the bootstrap capacitor used is $\mathrm{C}_{\text {bootstrap }}=0.22 \mu \mathrm{F}$ and the Sychronous MOSFET pulse train is 8 pulses long allowing the bootstrap capacitor enough time to charge. 

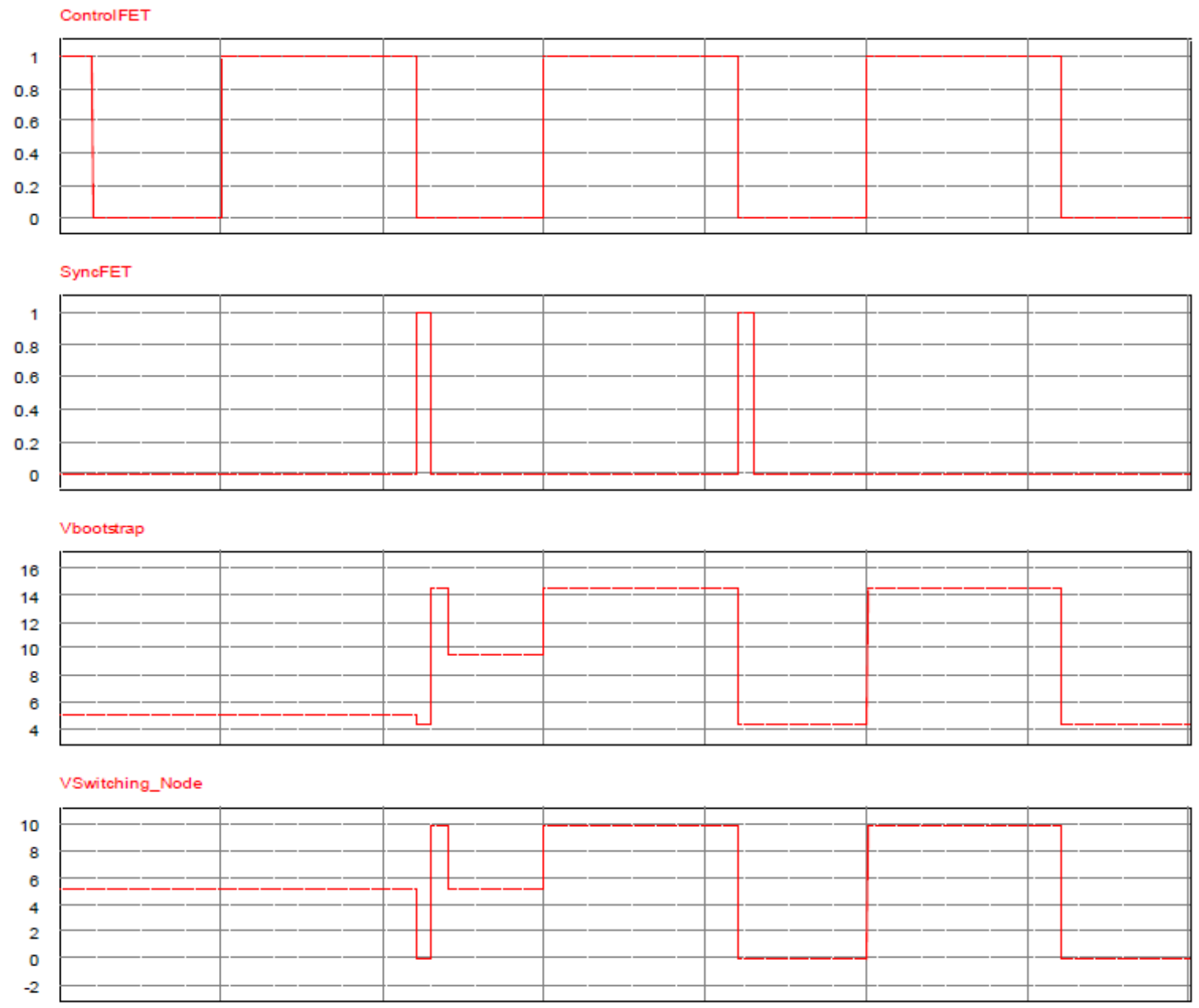

I(Lbuck)

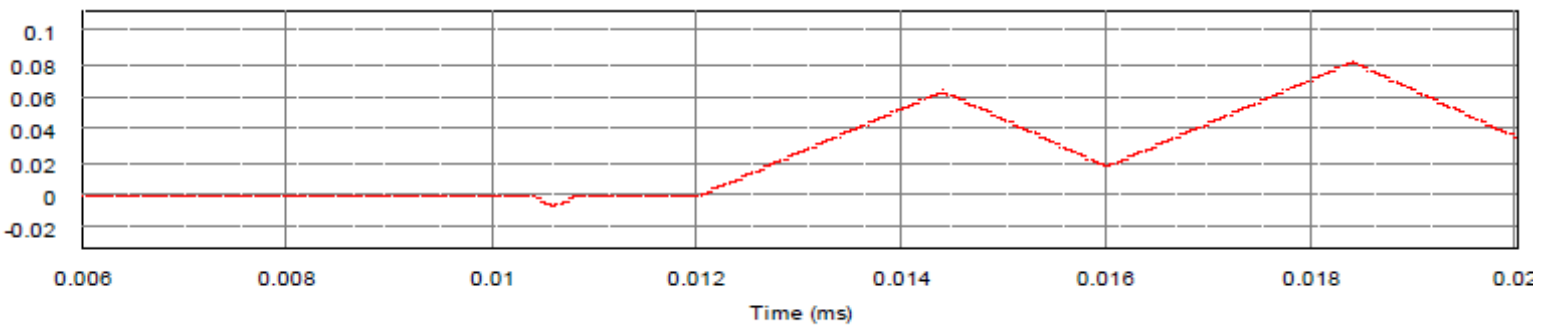

Figure 4-15 - Synchronous PulseStart to charge Bootstrap Capacitor

The synchronous MOSFET $\mathrm{Q}_{2}$ cannot initially be driven with the complementary signal of $\mathrm{Q}_{1}$ as this would cause a large negative inductor current and power would flow in the wrong direction. 
Due to the small duty cycle of $5 \%$ the inductor current is only slightly negative, shown in the Figure 4-15 waveform, I(Lbuck).

The operation of the Synchronous Pulse Start can be seen by the experimental waveform capture in Figure 4-16. The control signal for the Control MOSFET, Ctrl FET, cannot drive Q1 until the synchronous MOSFET, Sync FET, has turned on allowing the bootstrap capacitor to charge, which allows the driver to drive Q1 as observed by the switching node voltage, Vswitch_Node in Figure 4-6. The duration of the Sync FET pulse train is 8 pulses long and the operation of the synchronous MOSFET resumes normal operation once the inductor current rises above the threshold level.

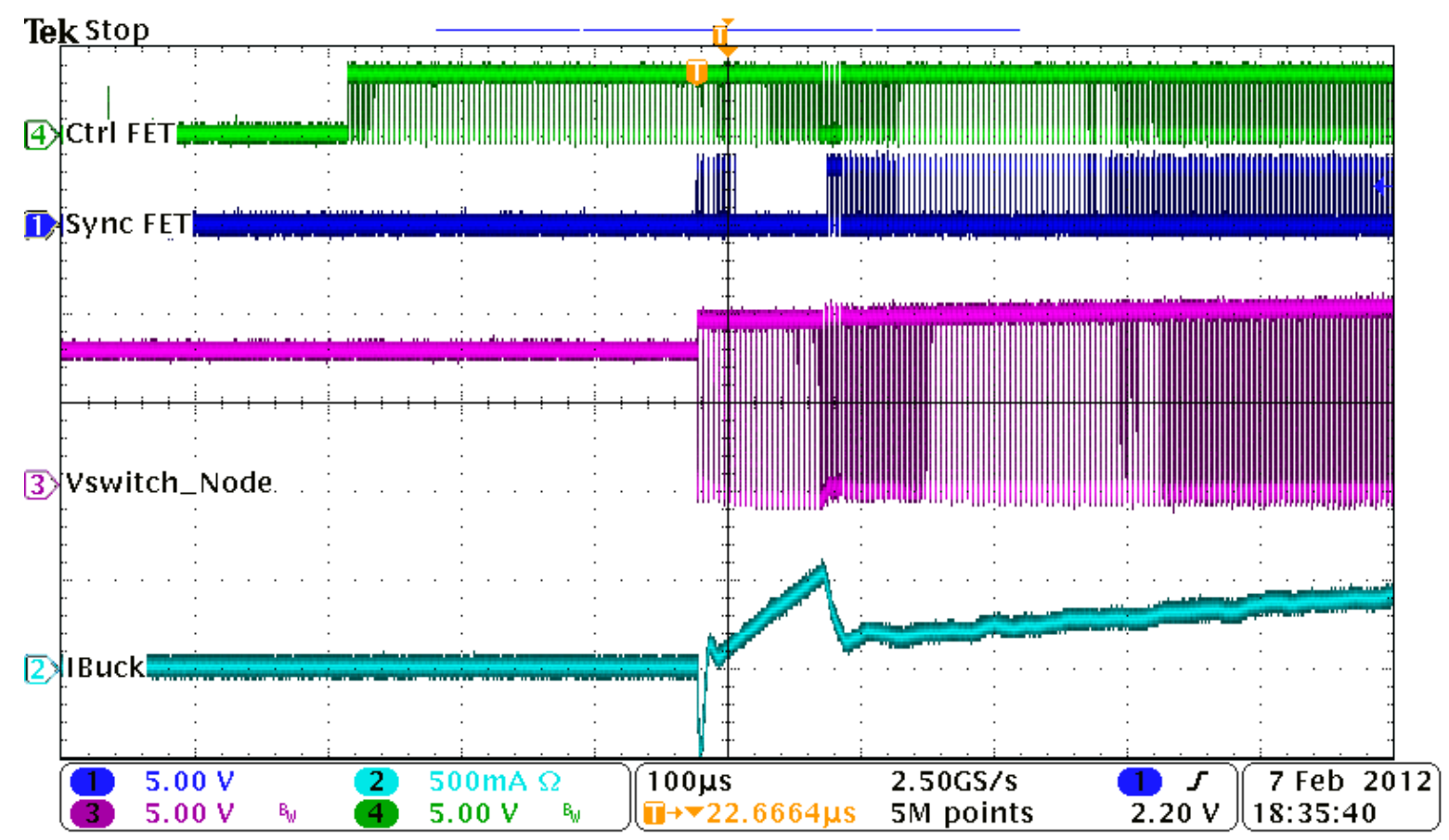

Figure 4-16 - Buck Synchronous Pulse Start Waveform 


\subsection{Prototype Requirement and Design}

In order to validate and fine tune the control theories presented in this chapter, a prototype voltage controlled and average current mode controlled Buck converter was designed with the following design parameters: $\mathrm{V}_{\text {in }}=6-80 \mathrm{~V}, \mathrm{~V}_{\text {out }}=5-13 \mathrm{~V}$ (limited to $5.6-8.4 \mathrm{~V}$ for a two cell system), fsw $=$ $250 \mathrm{kHz}, \mathrm{L}_{\text {Buck }}=150 \mu \mathrm{H}, \mathrm{C}_{\text {Buck }}=90 \mu \mathrm{F}, \mathrm{R}_{\text {sense }}=0.02 \Omega, \mathrm{I}_{\text {out }}=0-4 \mathrm{~A}$ (limited to $2 \mathrm{~A}$ when used with 2000mAh battery pack), $\mathrm{P}_{\text {outpeak }}=52 \mathrm{~W}$ (limited to $16.8 \mathrm{~W}$ for two cell battery pack used). The same TMS320F2808 digital signal processor (DSP) that was used for the Boost converter is also used for the control of the Buck converters digital controllers. As the TMS320F2808 DSP is overpowered for this application both the Boost and Buck digital controllers can be controlled on the same DSP with computational power to spare. The Buck converter’s Maximum Energy Extraction and Charge Control controllers are realized in the digital domain adding the benefits of quicker tuning of the digital controllers as well as other benefits of digital control. The power stage of the Buck converter was based on the continuous conduction mode Buck design equations found in [33].In order to keep the bill of materials small, the number of different components was limited and similar components were used in both the Boost converter and in the Buck converter. The parts used in the Buck converter are listed in Table 4-1.

Table 4-1 - Table of Components used in Buck Converter

\begin{tabular}{|l|l|l|l|}
\hline Part & Part Number & Manufacturer & Value \\
\hline Control MOSFET & NTD6416ANT4G & ON Semiconductor & $100 \mathrm{~V}, 17 \mathrm{~A}, \mathrm{Rds} 81 \mathrm{~m} \Omega$ \\
\hline Synchronous MOSFET & IPD70N10S3-12 & Infineon Technologies & $100 \mathrm{~V}, 70 \mathrm{~A}, \mathrm{Rds} 11.1 \mathrm{~m} \Omega$ \\
\hline Inductor & PCV-0-154-05 & Coilcraft & $150 \mu \mathrm{H}, 5.2 \mathrm{~A}$ RMS, Dcr $0.065 \Omega$ \\
\hline Driver & LTC4444IMS8E-5\#PBF & Linear Technology & $114 \mathrm{~V}$ Bootstrap, $1.4 \mathrm{Amp}$ \\
\hline Sense Resistor & LVK12R010DER & Ohmite & $\mathrm{R}=0.020 \Omega$ \\
\hline Op Amp & OPA2335AIDR & Texas Instruments & GBW $2 \mathrm{MHz}$ \\
\hline
\end{tabular}




\begin{tabular}{|l|l|l|l|}
\hline Decoupling Capacitor & ECW-U1105KCV & Panasonic - ECG & $1 \mu \mathrm{F}, 100 \mathrm{~V}$, Polyethylene \\
\hline DSP & TMS320F2808 & Texas Instruments & \\
\hline Battery & $5854602000 \mathrm{mAh}$ & UnionBattery & $2000 \mathrm{mAh}, 3.7 \mathrm{~V}$ \\
\hline
\end{tabular}

A schematic and printed circuit board (PCB) was designed with Altium Designer 10 [25]. The PCB was populated and debugged in the lab and a photo of the PEM prototype is shown in Figure 4-17.

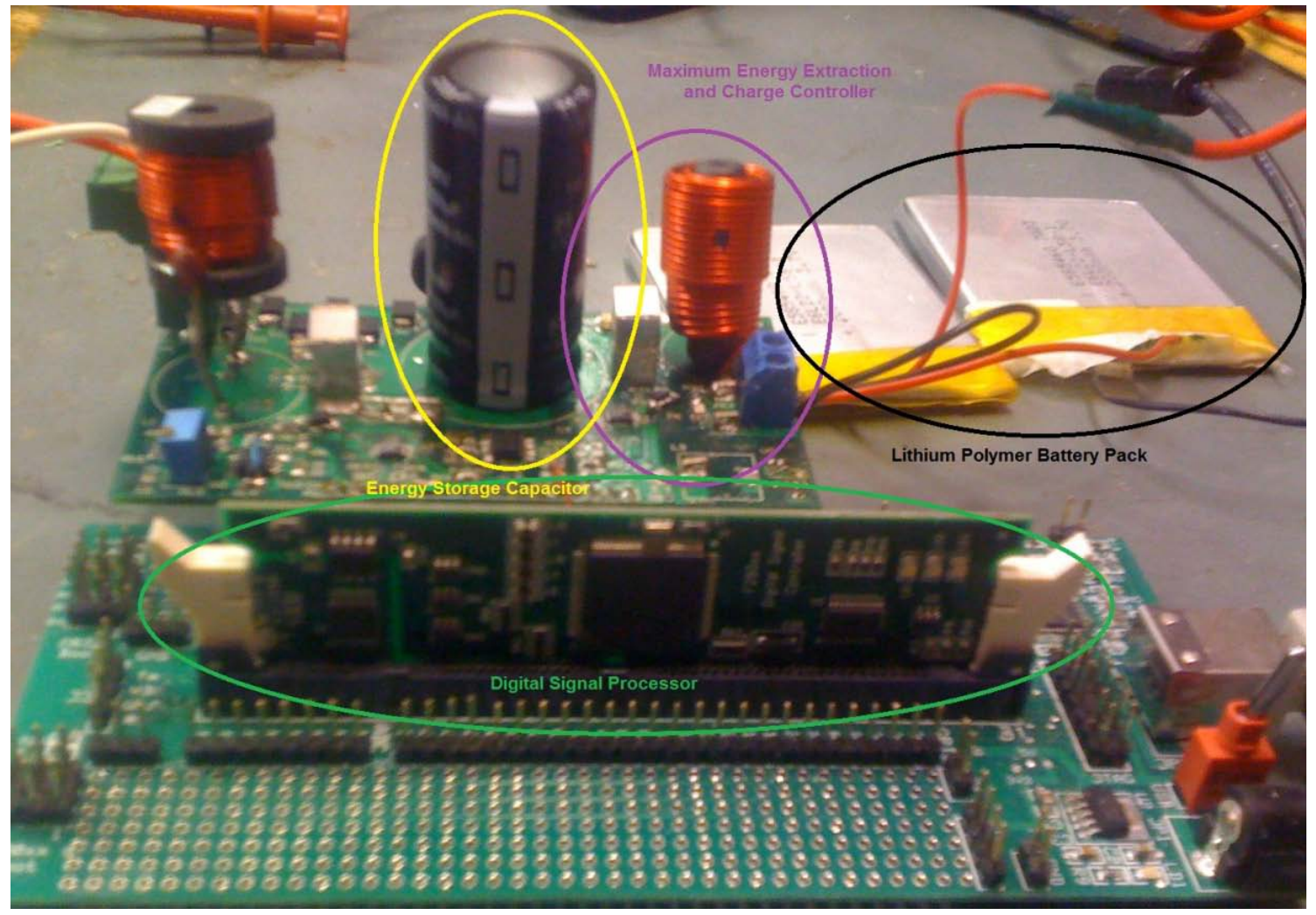

\section{Figure 4-17 - Photo of Prototype of Buck Maximum Energy Extraction and Charge}

\section{Controller}

As both the Boost controller and Buck controllers are running on the same DSP, the clock

frequency is the same running at $100 \mathrm{MHz}$ with the Buck switching frequency equal to $250 \mathrm{kHz}$

with the onboard ADC sampling each signal at $125 \mathrm{kHz}$. The Buck Maximum Energy Extraction 
and Charge Controller uses the sensed values for $\mathrm{V}_{\text {buck }}, \mathrm{I}_{\text {buck }}, \mathrm{V}_{\text {boost }}$ and $\mathrm{V}_{\text {rect }}$ with $\mathrm{I}_{\text {buck }}$ sensed through a current sense amplifier.

The sensed values are used in the various parts of the controller as outlined in Section 4.2. The digital controllers are calculated at $125 \mathrm{kHz}$. The derivation and design of the voltage and current compensators has been covered in the previous section with the current loop bandwidth equal to 11 $\mathrm{kHz}$ and the voltage loop bandwidth equal to $500 \mathrm{~Hz}$.

\subsection{Experimental Verification and Discussion}

This section will show experimental waveforms of the PEM with the Buck converter operating in both constant current and constant voltage charging modes with varying input power levels to the $\mathrm{R}_{\text {in }}$ Controlled Boost Converter to demonstrate the prototype and controllers effectiveness at meeting the design requirements set out in Chapter 2.

The first waveform capture is shown in Figure 4-18 with the following operating conditions, $\mathrm{V}_{\text {in }}=17.6 \mathrm{~V}$ peak, $\mathrm{F}_{\text {in }}=1.85 \mathrm{~Hz}, \mathrm{R}_{\mathrm{in}}=22 \Omega, \mathrm{I}_{\text {in }}=0.8$ A peak, $\mathrm{P}_{\text {inmax }}=14 \mathrm{~W}, \mathrm{P}_{\text {inavg }}=3.2 \mathrm{~W}$. The waveform capture is demonstrating the Buck converter charging the batteries with $\mathrm{P}_{\text {inmax }}<\mathrm{P}_{\text {outmax }}$. The Figure shows the waveforms $I_{i n}, V_{\text {boost }}, V_{\text {batt }}$ and $I_{\text {charge }}$ as labeled in the figure. From the input current waveform, $\mathrm{I}_{\mathrm{in}}$, we observe that the Boost converter is operating as expected in the Variable $\mathrm{R}_{\mathrm{in}}$ Control Mode. The emulated resistance of the Boost converter was set to $22 \Omega$ and the input current is approximately 0.8 Amps peak with a max input power of 14 watts and an average input power of approximately 3.2 watts. We can conclude from this waveform capture that the peak input power $\mathrm{P}_{\text {inmax }}$ is less than $\mathrm{P}_{\text {outmax }}$ from the shape of the battery charging current as the current has not reached the maximum charging current limit of 2A during this peak input power. The voltage rise of the ESC, $\mathrm{V}_{\text {Boost }}$ is caused by the rising input voltage, requiring the minimum voltage of $\mathrm{V}_{\text {Boost }}$ to increase so that the Boost converter can properly regulate the input current to the PEM. Any energy 
that is stored in the ESC due to this voltage rise is transferred to the battery pack once the input voltage level decreases causing the minimum voltage requirement of $\mathrm{V}_{\text {boost }}$ to decrease. As the charging current to the battery, $\mathrm{I}_{\text {charge }}$ is less than $2 \mathrm{~A}$, and the charging voltage of the battery, $\mathrm{V}_{\text {batt, }}$, is less than $8.4 \mathrm{~V}$, we can conclude that the battery is being charged within the charging requirements of the battery pack. The Buck MEECC is effective at charging the batteries within the charging constraints as well as charging the battery pack will all the energy that is extracted from the generator.

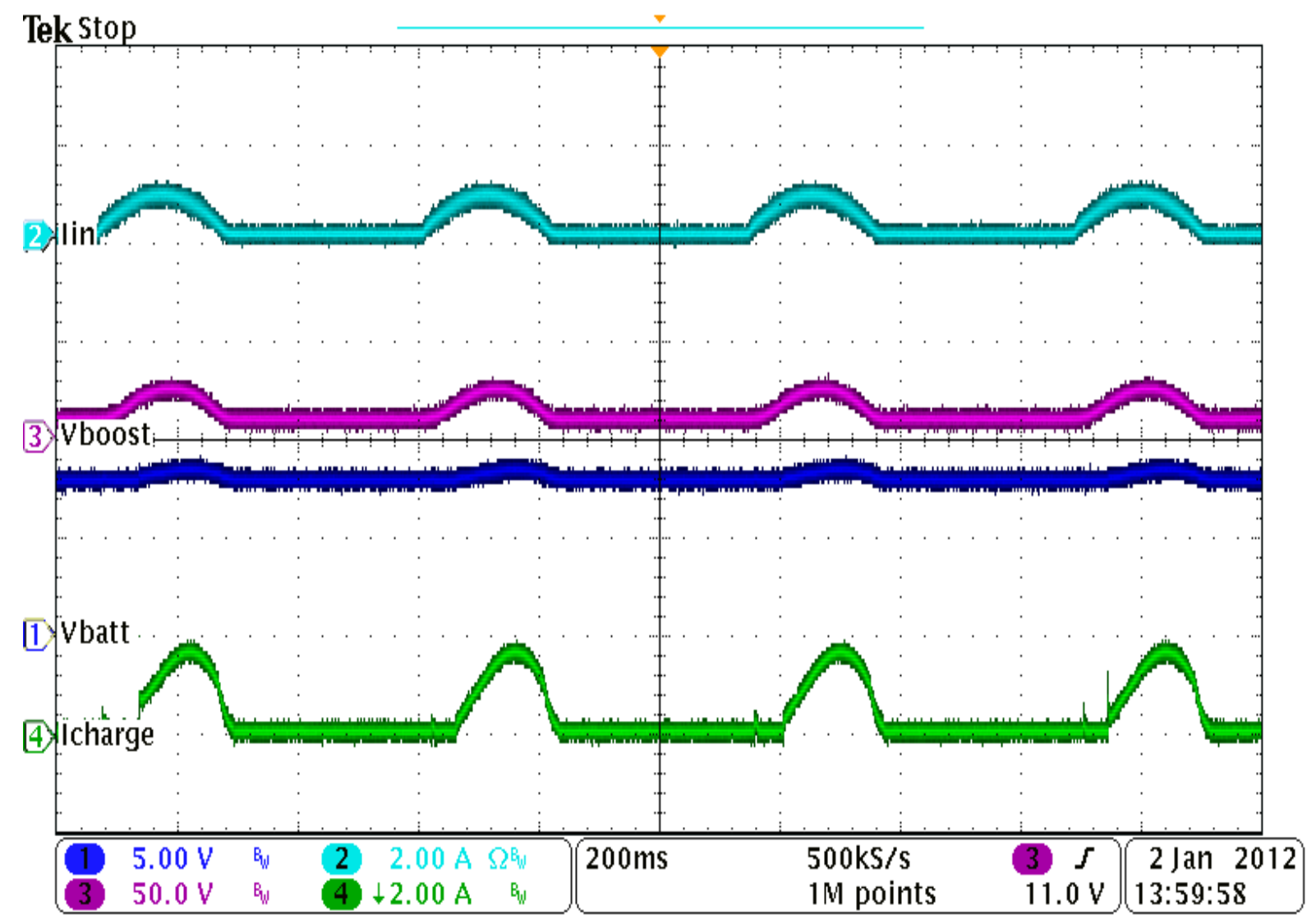

Figure 4-18 - Buck Charging with $\mathbf{P}_{\text {in }}<\mathbf{P}_{\text {outmax }}$

The next oscilloscope waveform capture, shown in Figure 4-19 is taken when the input power has increased and the instantaneous input power is greater than the peak output power. The PEM is 
operating under the following conditions, $V_{\text {in }}=30 \mathrm{~V}$ peak, $F_{\text {in }}=1.85 \mathrm{~Hz}, R_{\text {in }}=15 \Omega, I_{\text {in }}=2$ A peak, $P_{\text {inmax }}=60 \mathrm{~W}, P_{\text {inavg }}=14 \mathrm{~W}$. From the input current waveform in can be observed that the Boost converter is still operating as expected and drawing current according to the emulated resistor value of $15 \Omega$ with a peak current of $2 \mathrm{~A}$. The average input power is 14 watts and the peak power is 60 watts, we would expect from this high peak power that the batteries will be charging at their maximum CC rate of 2A and that the ESC is needed to store the excess peak power. When looking to Figure 4-19 battery charging current waveform, $\mathrm{I}_{\text {charge}}$, it can be observed that the battery is in fact charging at the CC rate of $2 \mathrm{~A}$ as well as the voltage in the ESC, $\mathrm{V}_{\text {boost }}$, is rising signifying the storage of energy. It can also be observed from the Icharge waveform that the batteries continue to charge at the maximum $\mathrm{CC}$ rate even after the input current waveform, $\mathrm{I}_{\mathrm{in}}$, has decreased to 0 . The energy that was stored in the ESC is now being transferred into the batteries and the batteries continue to charge at the CC rate until the energy in the ESC has been depleted. The batteries receive a charging current that is almost a pure DC current. 


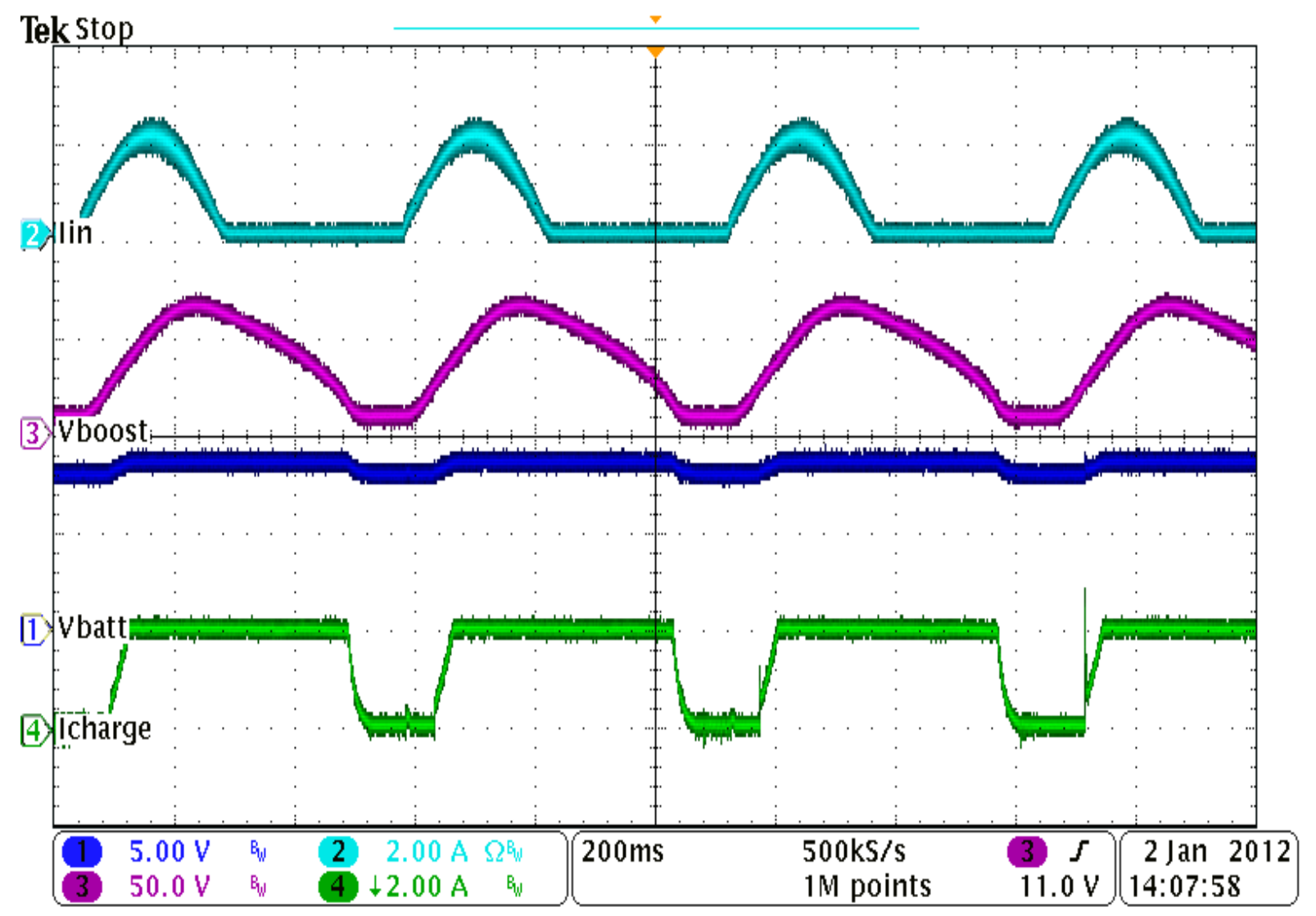

\section{Figure 4-19 - Battery charging in CC mode with higher $P_{\text {in }}$}

As the battery pack charges in CC mode the voltage level of the battery will slowly increase until it reaches a value of $8.4 \mathrm{~V}$ and the charger must transition into Constant Voltage charging mode. The next experimental waveform capture demonstrates this mode of charging, shown in Figure 4-20. The PEM is operating under the following conditions, $V_{\text {in }}=20 \mathrm{~V}$ peak, $F_{\text {in }}=1.85 \mathrm{~Hz}, \mathrm{R}_{\text {in }}=15 \Omega$, $\mathrm{I}_{\text {in }}=1.3$ A peak, $\mathrm{P}_{\text {inmax }}=26 \mathrm{~W}, \mathrm{P}_{\text {inavg }}=5.4 \mathrm{~W}$. Looking at the Boost input current waveform, $\mathrm{I}_{\mathrm{in}}$; it can be observed that the Boost converter is still emulating a constant resistor while the Buck converter is charging the battery in CV mode. The Boost converter is emulating a resistor value of $15 \Omega$ with a peak input current of $1.3 \mathrm{~A}$. The peak input power is 26 watts with the average power around 5.4 watts. As the peak input power is greater than the maximum charging power we expect that the batteries will be charging in either CC or CV mode, in this case because the batteries are nearing 
their voltage limit of 8.4 Volts it will be CV mode as determined by the controller. As the peak input power is greater than the output power to the battery pack the ESC has an increase in voltage as shown by the $\mathrm{V}_{\text {boost }}$ waveform.

It can be observed that the Buck is charging in CV mode based on both the battery charging current waveform, $\mathrm{I}_{\text {charge, }}$ and the charging voltage, $\mathrm{V}_{\text {batt }}$. As the charging current increases the charging voltage increases as well until the voltage of the battery hits the CV limit of 8.4 Volts at which point the charging current is reduced to maintain the constant voltage charge. The battery continues to charge at the constant voltage until the energy that was stored in the ESC has been depleted. Similar to the previous figure, the ESC allows the battery to continue to charge at the full rate even after the input current has decreased to zero. 


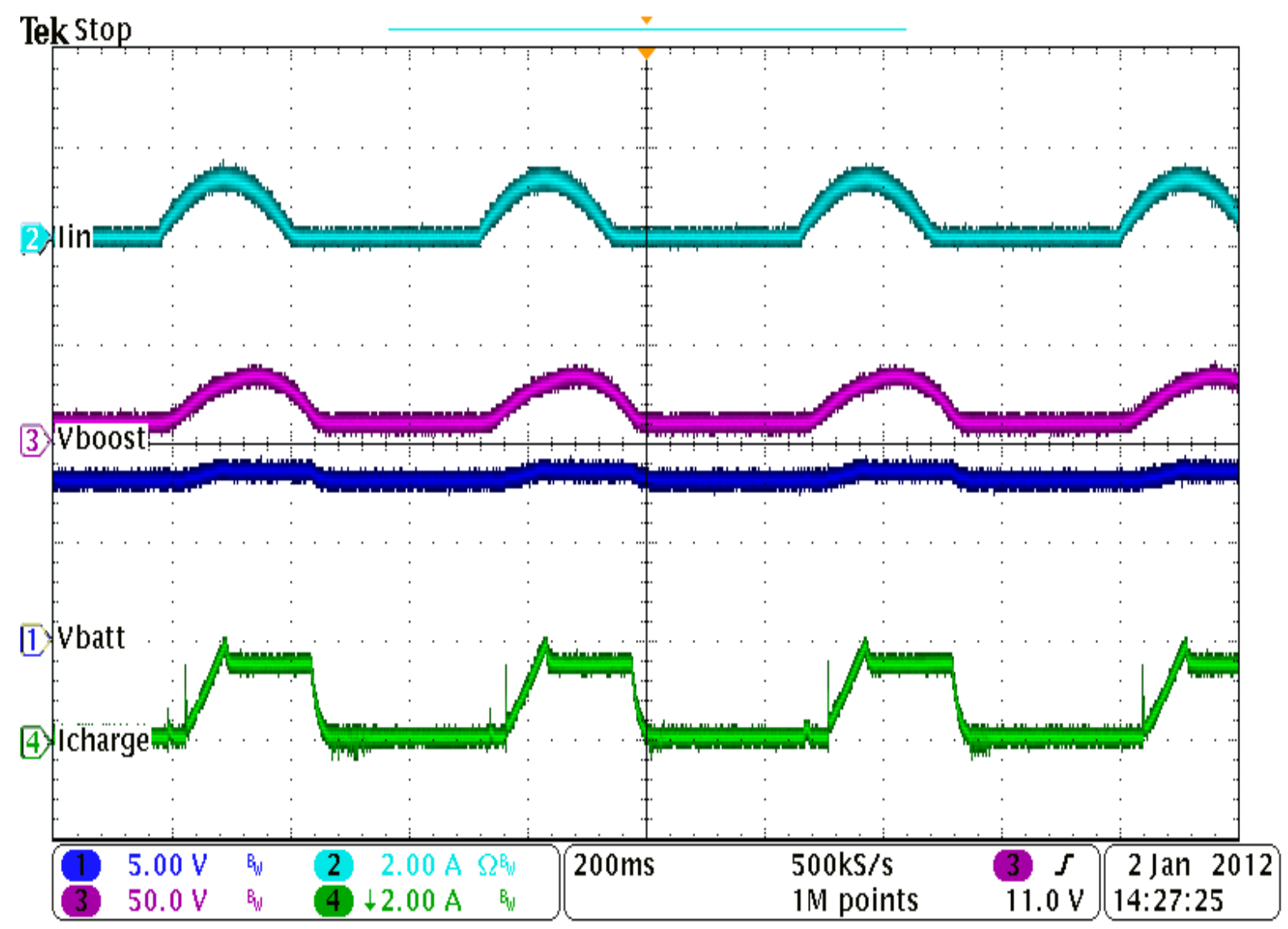

Figure 4-20 - Battery charging in CV mode

From the experimental waveforms it can be concluded that the MEECC is effective at controlling the Buck converter to charge the batteries within their charging limits at the same time as extracting the maximum amount of available power from the ESC and into the batteries.

The DC efficiency of the Buck converter was measured with the Buck converter operating in Constant Current charging mode and is shown in Figure 4-21. The operating condition of the Buck converter is changed by varying the $R_{\text {in }}$ value of the Constant $R_{i n}$ controlled Boost converter while keeping the input voltage constant at $V_{\text {in }}=20 \mathrm{~V}$ DC, the value of $\mathrm{R}_{\text {in }}$ is shown in the figure. The efficiency is measured from the input power of the Buck converter to the output charging power of the battery pack. The input voltage of the Buck converter, $\mathrm{V}_{\text {boost }}$, is determined by the MEECC 
controller and is shown in the figure as the red curve. The output voltage of the Buck converter is determined by the SoC of the battery as well as the charging level. The input power level of the Boost converter is shown for a reference so that the efficiency of the two converters can be compared and is shown in the figure along the x-axis. The efficiency of the Buck converter is shown verses the input power level of the Boost converter.

The peak efficiency of the Buck converter was measured at 93.7\% with the Boost converter emulating $\mathrm{R}_{\mathrm{in}}=20 \Omega$ at an input power of 18 watts. It should be noted that in the efficiency measurement with $\mathrm{R}_{\mathrm{in}}=15 \Omega$ and $\mathrm{P}_{\text {in }}=25.7 \mathrm{~W}$ the output power level to the battery pack is higher than rated for the $2000 \mathrm{mAh}$ battery pack used, so a larger battery pack was substituted in so that the measurement could be made with the higher output power.

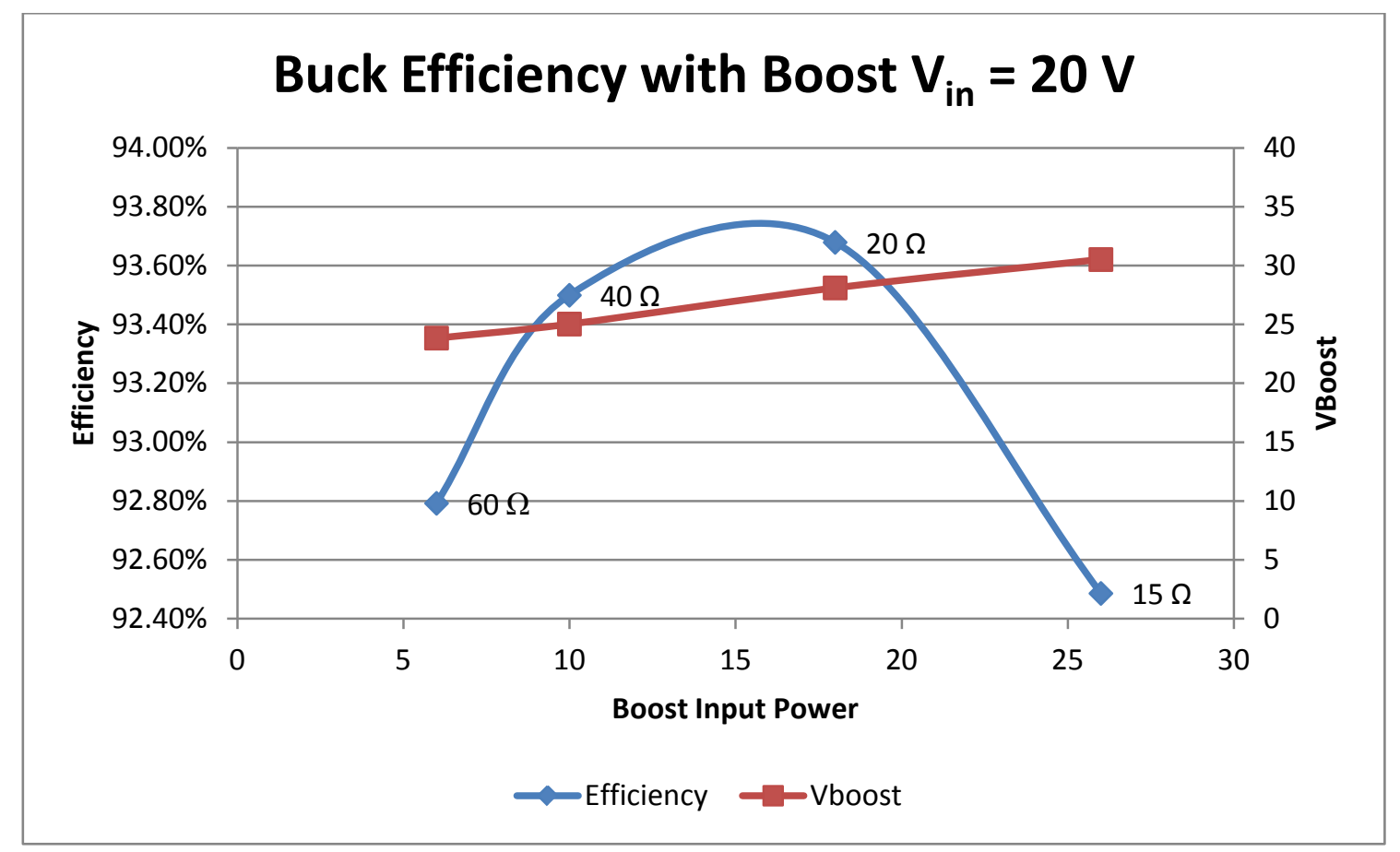

Figure 4-21 - DC Efficiency of Buck Converter in CC Mode 
As was the case with the efficiency measurement of the Boost converter, this measurement is made with a DC input voltage and only represents a single point along the input voltage curve. A total system efficiency with an AC input voltage waveform will be discussed in Section 5.4.

\subsection{Conclusion}

A solution to the second part of the Power Electronics Module design requirements, to minimize the voltage and therefore energy stored in the ESC by the control of the battery charging current while staying within the charging limitations of the battery was presented in this chapter. The solution to this design requirement is the Maximum Energy Extraction and Charge Controller. This controller contains four controller blocks consisting of the Buck Average Current Controller, the Buck Voltage Controller, the Boost Voltage Controller, and the Vboostmin Controller with the first two controllers controlling the charging conditions of the battery pack, and the last two controlling the voltage of the ESC to its minimum value. The small signal transfer function from duty cycle to inductor current was derived for the Buck average current mode control using block diagrams along with the DC Gain of the transfer function. As in the case with the Boost converter the Buck average current mode Buck controller can make use of the Voltage Adaptive Gain compensator to normalize the DC Gain of the transfer function over the entire input voltage range. The low input voltage bandwidth was increased from $1.66 \mathrm{kHz}$ to $11 \mathrm{kHz}$ with a phase margin of 62.4 degrees, with the high input voltage bandwidth equal to $11 \mathrm{kHz}$ with a phase margin of 75 degrees with the use of the Voltage Adaptive Gain controller. The Voltage Adaptive Gain compensator reduced the required turn on time from $13 \mathrm{~ms}$ to $3.12 \mathrm{~ms}$. The small signal transfer function from inductor current to output voltage was also derived from the block diagram in order to design the digital voltage loop compensator. The bandwidth of the voltage loop was chosen at $500 \mathrm{~Hz}$ with a phase margin of 86.6 degrees. A method of driving the synchronous MOSFET to allow the control 
MOSFET to turn on without the use of an isolated supply is presented. A digitally controlled prototype was designed and built on a Printed Circuit Board to test and validate the function of the MEECC controllers. Experimental results were captured by the oscilloscope to ensure the proper operation of the circuit and controllers for the different input power levels and charging modes of the battery. The DC efficiency of the Buck converter was measured with the Boost converter emulating different resistor values with an input voltage of $20 \mathrm{~V} \mathrm{DC}$ to the Boost converter. The power level ranged from 6 to 26 watts and the peak efficiency of the Buck converter was measured at $20 \Omega$ with an input power level of 18 watts at an efficiency of $93.7 \%$. 


\section{Chapter 5}

\section{System Design}

\subsection{Introduction}

This chapter will discuss the system design of the PEM. The system design will cover the other parts to the design that were not covered in Chapters 3 and 4. In this chapter the embedded system software and timing of the code will be discussed. A selection guide for the size and voltage of the ESC vs. the peak input power will follow. Experimental waveforms of the $\mathrm{R}_{\text {in }}$ Controlled Boost converter and Maximum Energy Extraction and Charge Control Buck converter are presented to demonstrate the PEM subsystems working together. A mechanical test rig is designed to emulate the input power profile that a human user would generate to the system while removing any inconsistency that a human would introduce into the experimental results. Using the mechanical test rig as an input, an experiment is carried out to determine how effective the PEM system is at extracting energy and storing it in the batteries verses a single stage design without intermediate energy storage capability. Finally this chapter will compare the efficiency of both converters as well as the total system efficiency of the PEM. The next section in this chapter will discuss the considerations when designing an embedded software solution along with the timing constraints when dealing with real time critical tasks.

\subsection{Embedded System Software Considerations and ISR Execution Timing}

When an embedded system is used to control real-time events, such as the DSP for the digital controllers in the case of the PEM, it is important for the software to be as efficient as possible. The timing and order of certain software tasks need to be appropriately arranged such that software 
events can occur on time to sync up with real time events that the embedded controller is monitoring or controlling. It is a common practice for the design engineers to optimize the code of the embedded system to reduce the size and cost of the processor needed in an attempt to reduce the total cost of the system [34].

In the case of the PEM for our embedded DSP the code also needs to be appropriately arranged and optimized in order to reduce the power consumption of the processor in effort to increase the power harvest. It has been mentioned earlier in the thesis that the sampling frequency is half that of the switching frequency of the converter. This has a few main reasons, the first being that the controllers do not need $25 \mathrm{kHz}$ of bandwidth that would require the ADC to sample at $250 \mathrm{kHz}$, we can get away with sampling at half of the switching frequency at $125 \mathrm{kHz}$ and still achieve a controller bandwidth of $11 \mathrm{kHz}$. As the ADC can sample at half the switching frequency, the power consumption of the ADC chip itself will be reduced. This also reduces the number of times the time critical code is executed by a factor of two, namely the ADC driving code as well as the Interrupt Service Routine (ISR) which contains all of the digital controllers' code.

The reduction of cycles needed per second would allow the clock frequency of the DSP to be reduced below the current clock frequency of 100MHz. Additional power saving can be found be disabling all of the peripherals that are not being used by the PEM such as the extra ePWM, eCAN, eCAP, I2C and other peripherals [35]. When comparing the power consumption before and after these extra modules were disabled, the power consumption of the DSP has been reduced from 1.2 watts to 0.91 watts for a power reduction of $21 \%$. If we were able to reduce the clock frequency of the processer due to the reduced number of cycles needed per second to $60 \mathrm{MHz}$ an estimated additional power savings of $23 \%$ could be achieved bringing the total power consumption of the 
DSP down to 0.7 watts. It was not, however, in the scope of this thesis to push the limits of optimizing the code to be able to reduce the clock frequency of the DSP for a power savings of 0.21 watts.

Parts of the main loop code and some of the software blocks for the ISR were used from Texas Instruments software library DSPLib as well as example code provided by Texas Instruments as a starting block to aid in the development on their DSPs [36]. To also aid in the development of the software extensive use of the hardware reference guides for the operation and control of the various peripherals were used. The reference guides that were used were the Analog-to-Digital Converter [37], CPU and Instruction Set [38], System Control and Interrupts [39], High Resolution Pulse Width Modulator [40], and the Enhanced Pulse Width Modulator [41].

In the software development stage, there still must be considerations in terms of the sequence and timing of the software events inside the ISR and the hardware events such as the sampling of the various signals by the ADC to limit the delay associated with the sample and hold process of the digital control. In order to visualize and optimize the execution of the ISR to ensure that the appropriate variables are updated before they are needed as well as to calculate the delay of each sampled signal, a timing diagram has been created and is shown in Figure 5-1. 


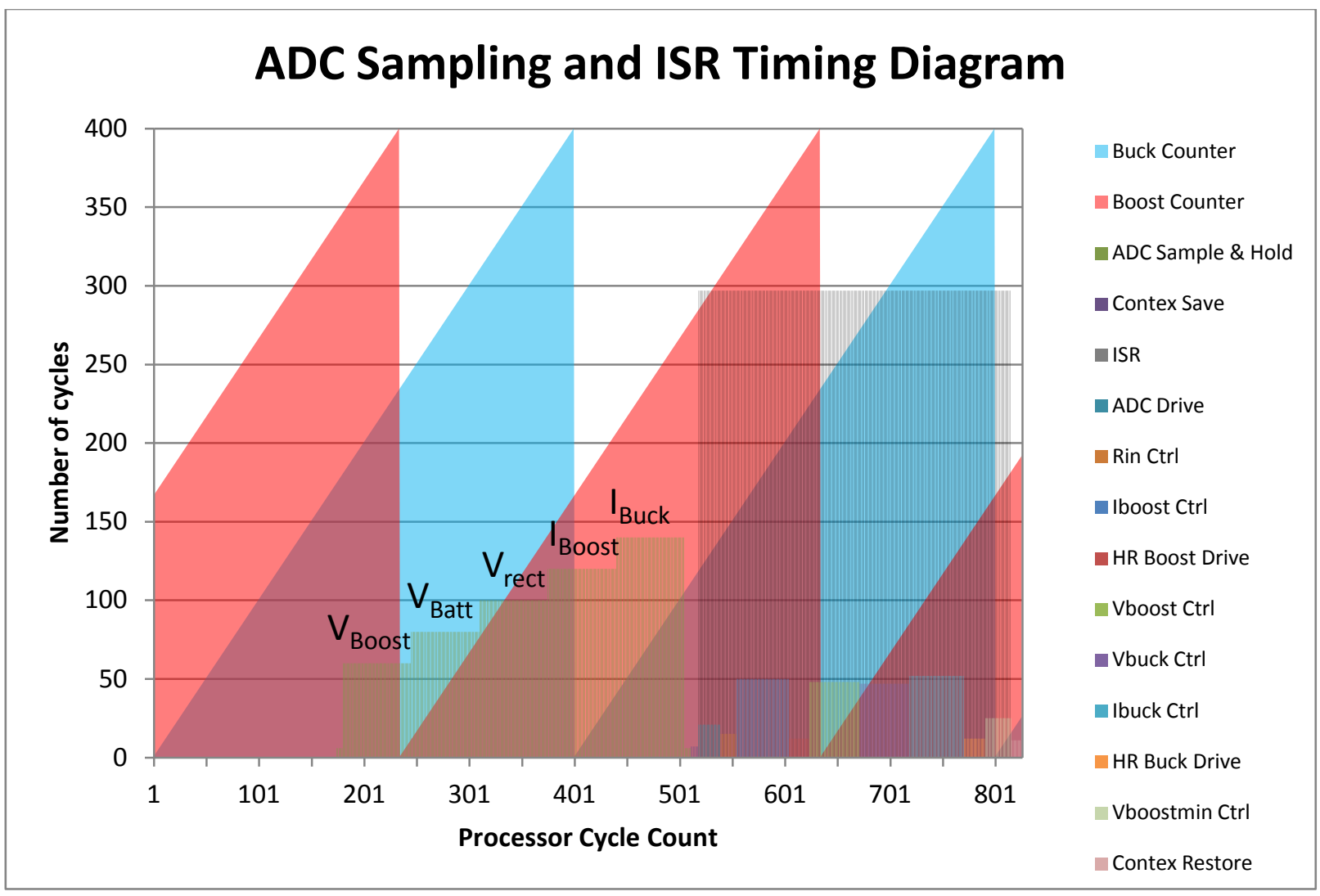

Figure 5-1 - ADC Sampling and Interrupt Service Routine Timing Diagram

The timing diagram is shown with the $\mathrm{x}$-axis representing the cycle count of the processor during normal execution and was created by calculating the difference in the processor cycle count before and after a segment of code was executed. The height of each block represents the number of cycles each segment of code takes to execute in processor cycles with the exception of the ADC Sample \& Hold in which the execution time of each sample is the same as the first sample of $V_{\text {boost }}$ equal to 65 cycles. The sequential samples are shown with exaggerated cycle counts to differentiate them from the previous samples. The value of each sample is captured by the sample and hold hardware of the ADC in the last cycle of each sample and hold period shown in the diagram. The start of conversion of the ADC is driven by an interrupt triggered by the period counter of the Boost converter equaling 341. After the ADC is finished sampling the five different signals, the ADC End 
of Sequence (EoS) triggers the ISR to start. The processor is not held up during the sampling of the signals, the ADC timing is configured through hardware registers by the DSP at startup. The processor is free to execute the main loop during this time.

The Buck period counter and Boost period counter are represented in the diagram as an increasing ramp and are reset to zero once they reach a cycle count of 400 representing a switching frequency of $250 \mathrm{kHz}$ with a $100 \mathrm{MHz}$ processor clock frequency. The Buck converter and Boost converter are synchronized with the Buck converter having a phase delay of 167 cycles, or $1.67 \mu$ s to lag the switching of the Buck converter behind the Boost converter which serves two main purposes. The first reason is that the Boost converters inductor current will flow to the output into the ESC during the off time of the Control MOSFET, the complementary signal of the duty cycle; this happens in the latter half of the switching period. The Buck will draw current from the ESC during the on time of its Control MOSFET, the positive portion of its duty cycle; this happens in the first half of its switching period. By delaying the switching period of the Buck converter, the output current from the Boost converter will have a direct path to the input of the Buck converter. A lower percentage of the high frequency current will need to be stored by the decoupling capacitors. This will reduce the RMS current through the capacitors, reducing the conduction losses associated with the ESR of the capacitors. The second reason for the phase difference between the Boost and Buck converter is so that the controllers can calculate the new control variables based on the newly sampled values with minimal delay from sample instant to when the PWM duty cycle is updated for both the Boost and Buck converters. 
The ISR that is called by the ADC EoS contains the sequence of code needed to execute the various controllers and a software flow diagram is provided in Figure 5-2 to show the code executed by the ISR.
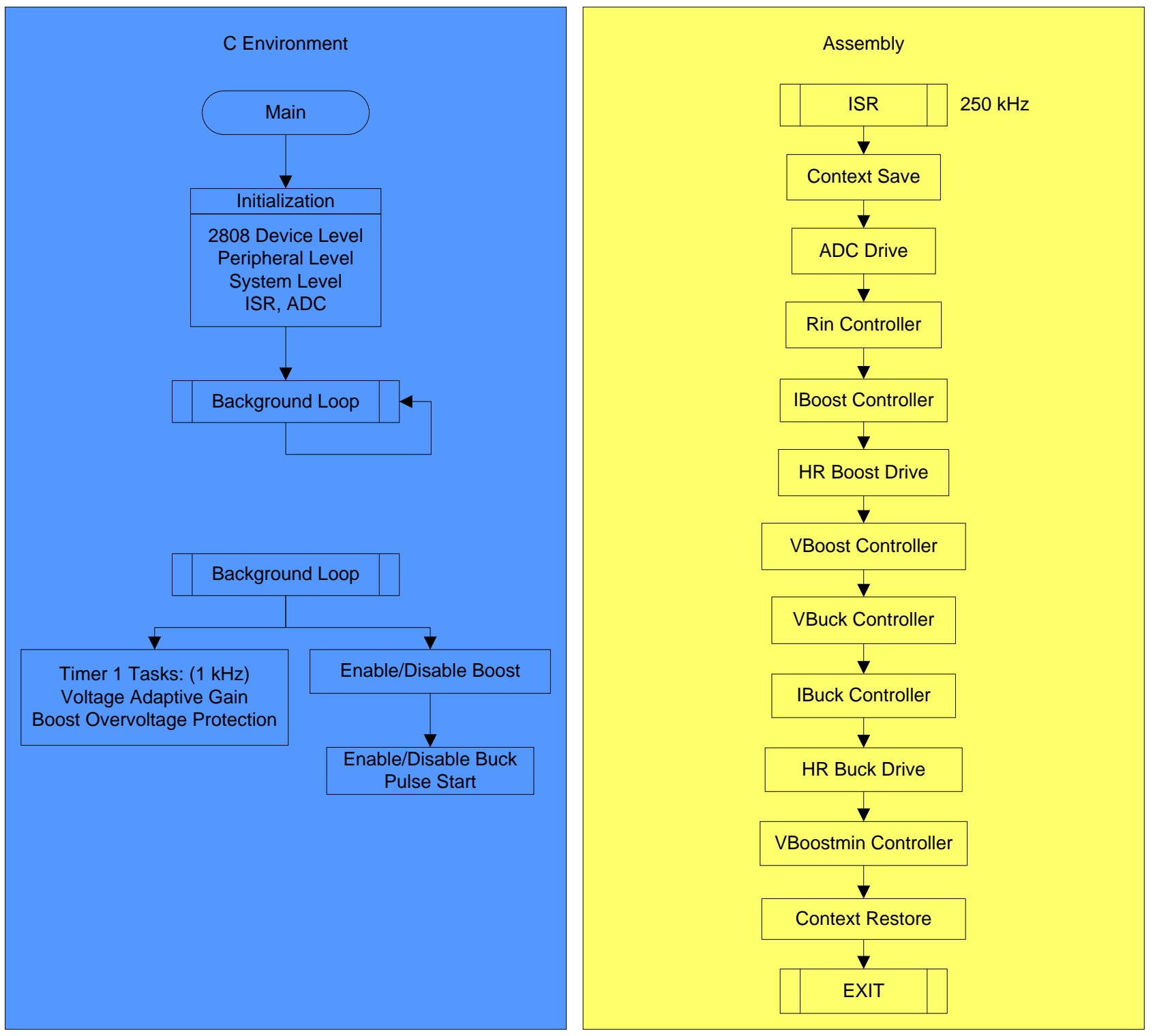

Figure 5-2 - Software Flow Chart for DSP 
Once the ISR is called by the EoS of the ADC the 'context save' code is executed, this saves the processor state and registers before any of the ISR code can be executed. Once in the ISR, the ADC Drive code is run, this code takes the values that were sampled by the ADC and updates the variables to the newly sampled values for use by the controllers that will be run later in the ISR. The Boost converter controllers are run which includes the $\mathrm{R}_{\text {in }}$ Controller, 'Rin Ctrl' and the Voltage Adaptive Gain two-pole two-zero average current mode controller, ‘'Boost Controller’. The duty cycle output from the average current mode controller is then updated to the High Resolution ePWM module by the HR Boost Drive code. As can be seen from the timing diagram in Figure 5-1, the HR Boost Drive code has finished executing and updates the duty cycle just before the next period of the Boost converter has started and the duty cycle will take effect in the upcoming switching cycle.

The Buck controllers, VBoost controller, VBuck controller and IBuck controller are all executed followed by the updating of the Buck duty cycle to the high resolution ePWM module by the HR Buck Drive code. Again the duty cycle is updated just before the next period of the Buck converter has started and the upcoming switching period will use the newly updated duty cycle.

The last controller to be executed in the ISR is the VBoostmin controller. It is run last as the sampled variables that are used in the controller are averaged over 32 sampling periods and are not as time sensitive as the other controllers. This prevents this controller code causing more delay for the other time sensitive controllers between the sampling instant and the updating of their values.

The last piece of code that is run by the ISR is the context restore which restores the registers and processor state to the pre ISR state so that the main loop can continue where it left off. 
Another critical piece of information that can be extracted from the timing diagram in Figure 5-1 is the sample and hold delay of the samples used in the controllers. As mentioned in Section 3.6, when designing a digital compensator the delay from the sample instant to when the output of the controller is updated needs to be considered in the control loop transfer function as this introduces a phase lag to the plant. If this delay is not accounted for when designing the digital compensator the actual phase margin will be less than the designed phase margin. This delay has be accounted for and added to the transfer function of the system by using equation (3.20) and the time delay of the sampled signals, $T_{d}$. The delay for the sampled variables, $T_{d}$ can be calculated from Figure $5-1$ by calculating the difference between the cycle count at the sample instant to when the duty cycle is updated; a table with the calculated delay is given in Table 5-1.

Table 5-1 - Delay of Sampled Signals from Sampling Instant to when they take Effect

\begin{tabular}{|l|l|l|}
\hline Sampled Signal & Delay for Boost Controller & Delay for Buck Controller \\
\hline $\mathrm{I}_{\text {Buck }}$ & Not used & 296 cycles $\left(\mathrm{T}_{\mathrm{d}}=2.96 \mu \mathrm{s}\right)$ \\
\hline $\mathrm{I}_{\text {Boost }}$ & 195 cycles $\left(\mathrm{T}_{\mathrm{d}}=1.95 \mu \mathrm{s}\right)$ & Not used \\
\hline $\mathrm{V}_{\text {rect }}$ & 260 cycles $\left(\mathrm{T}_{\mathrm{d}}=2.6 \mu \mathrm{s}\right)$ & 426 cycles $\left(\mathrm{T}_{\mathrm{d}}=4.26 \mu \mathrm{s}\right)$ \\
\hline $\mathrm{V}_{\text {batt }}$ & Not used & 491 cycles $\left(\mathrm{T}_{\mathrm{d}}=4.91 \mu \mathrm{s}\right)$ \\
\hline $\mathrm{V}_{\text {Boost }}$ & 391 cycles $\left(\mathrm{T}_{\mathrm{d}}=3.91 \mu \mathrm{s}\right)$ & 557 cycles $\left(\mathrm{T}_{\mathrm{d}}=5.57 \mu \mathrm{s}\right)$ \\
\hline
\end{tabular}

The order in which the signals are sampled as well as the layout of the code in the ISR has been chosen in attempt to minimize the delay of the more time sensitive signals. The time sensitive signals, $\mathrm{I}_{\mathrm{buck}}$ and $\mathrm{I}_{\mathrm{boost}}$, are the ones used in the high bandwidth controllers, namely the average current mode controllers for both the Buck and Boost converters which have a bandwidth around $11 \mathrm{kHz}$. The sampling delay has more of an effect on the higher bandwidth controllers than it does on the lower bandwidth controller such as the voltage loop controller of the Buck converter. The 
sampled signal for the voltage loop controller, $\mathrm{V}_{\text {batt }}$ is placed lower on the priority list as the longer delay has a less significant effect on the phase margin of the $500 \mathrm{~Hz}$ bandwidth controller.

\subsection{Selection of the Size and Voltage of the Energy Storage Capacitor versus Input Power}

One of the key parameters that need to be selected in the design process for the two stage PEM is the size of the Energy Storage Capacitor. The selection of the capacitor has some design tradeoffs between capacitance and maximum voltage requirement of the capacitor, the larger the capacitor value, the less voltage variation that is needed to store the same amount of energy. We want to design the capacitor size and voltage such that while charging the battery in CC mode we are able to store all of the excess energy that cannot be transferred to the battery during one input voltage cycle. The worst case condition for this will happen when the peak input power is high and the output power to the battery, $\mathrm{P}_{\text {battmin, }}$, is low. During the CC cycle the power output to the battery will be the lowest when the battery's SoC is lowest because the battery voltage will be at its minimum value and the charge current does not vary in the CC cycle.

A design chart has been created, showing the selection tradeoff between $V_{\text {boostmax }}$ and the value of the capacitor is shown in Figure 5-3. The chart was created with data calculated using Matlab and a custom program that calculates the voltage level of the ESC based on iterative equations.

The parameters used to calculate the design chart were based on typical values for a human walking at $1.5 \mathrm{~m} / \mathrm{s}$ and are $\mathrm{V}_{\text {rect }}=28$ Volts peak, $\mathrm{F}_{\text {in }}=1.85 \mathrm{~Hz}$ AC, $\mathrm{P}_{\text {outmin }}=12.8$ watts based on two Lithium Polymer cells with a capacity of $2000 \mathrm{mAh}$. To vary the input power the input voltage is kept constant while $\mathrm{R}_{\text {in }}$ is varied from $60 \%$ full load to $120 \%$ full load (28.3 $\Omega-14.17 \Omega$ ) with 17 
$\Omega$ used for full load, $\mathrm{P}_{\text {inavg }}=11.5$ watts, $\mathrm{P}_{\text {inpeak }}=50 \mathrm{~W} . \mathrm{P}_{\text {outmin }}$ is calculated using equation (5.1) with the following parameters: Cells $=2, \mathrm{~V}_{\min }=2.8 \mathrm{~V}, \mathrm{R}_{\text {batt }}=0.2 \Omega, \mathrm{I}_{\mathrm{CCMAX}}=2 \mathrm{~A}$

$$
P_{\text {outmin }}=\mathrm{N}_{\text {cells }} *\left[\text { Vmin }+ \text { Rbatt } * I_{\text {CCMAX }}\right] * I_{C C M A X}
$$

where $\mathrm{N}_{\text {cells }}$ is the number of batteries, $\mathrm{V}_{\min }$ is the voltage when the battery is at its minimum SoC, $\mathrm{R}_{\text {batt }}$ is the internal resistance of the battery, $\mathrm{I}_{\text {CCMAX }}$ is the $1 \mathrm{C}$ charging current based on the capacity of the battery.

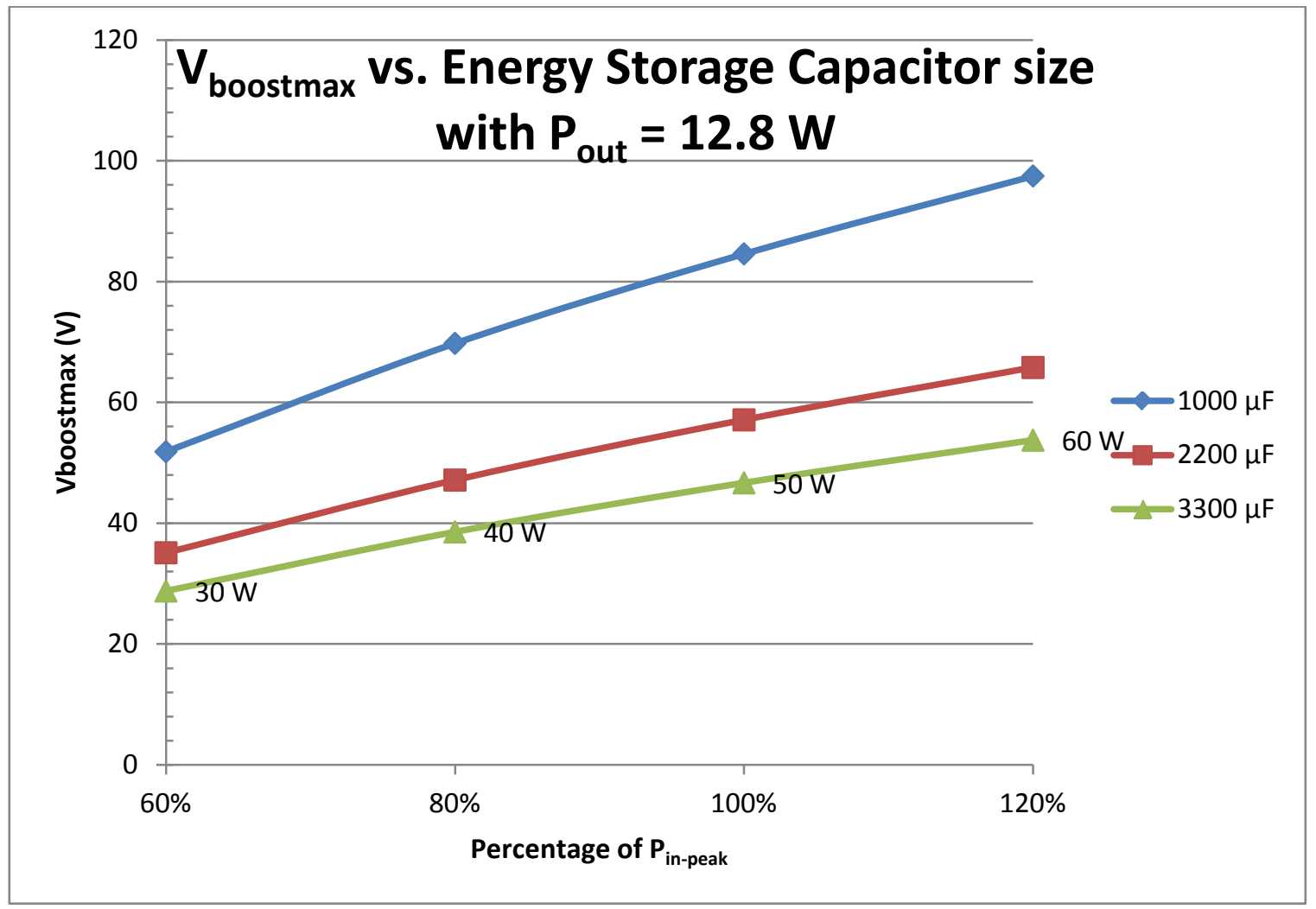

Figure 5-3 - Selection guide for $V_{\text {boostmax }}$ vs. Energy Storage Capacitor size with Pout $=12.8$ watts

As the value of the capacitor is reduced, $\mathrm{V}_{\text {Boostmax }}$ must increase to store the equivalent energy for a given power level. The amount of energy stored for a given capacitor and voltage is shown in 
equation (5.2)

$$
E=\frac{1}{2} C V^{2}
$$

where $\mathrm{E}$ is the energy stored in the capacitor in Joules, $\mathrm{C}$ is the capacitance in Farads, $\mathrm{V}$ is the voltage of the capacitor. In determining the best trade off for voltage and size of capacitor, it can also be noted that the higher voltage will require higher voltage rated MOSFETs which will have larger losses decreasing the overall system efficiency as well as both converters are more efficient at lower step up and step down ratios.

Based on the analysis, the value of ESC is selected as $2200 \mu \mathrm{F}$. This will provide an ESC capable of storing the excess energy with an input power of $120 \%$ of the nominal input power level while not exceeding $65 \mathrm{~V}$ for $\mathrm{V}_{\text {boostmax }}$ when using the two 2000mAh battery pack charging in CC mode.

\subsection{Experimental Verification}

In order to verify that the $\mathrm{R}_{\mathrm{in}}$ Controlled Boost converter and the Buck Maximum Energy Extraction and Charge Controller operate well together and meet the overall requirements of the PEM design the complete system needs to be tested. The design goals that were set out in the earlier chapters will be tested to ensure that both systems can work together to achieve these goals. The three main design goals are as follows:

1. To extract current from the generator in any user specified profile

2. To charge the batteries with all of the extracted power while staying inside the charging specifications of the batteries

3. To extract the maximum permissible energy that is available from the generator.

The first two design criteria can be easily shown with experimental waveforms. The third design requirement will require a more in depth experiment and test setup to show that the system can 
effectively extract more power from the generator than an equivalent single staged system which will be discussed in the next section. The Boost converter has been shown in previous sections to draw current in any user desired profile; we must now only prove that during this power extraction the battery is charging within the battery specifications. The first charging specification of the battery is that the charging current of the battery must not exceed the $1 \mathrm{C}$ charging rate for the battery, or 2 Amps for the 2000mAh batteries we are using.

Shown in Figure 5-4 is the full system operating waveforms, $I_{i n}, V_{\text {boost }}, V_{\text {batt }}$, and $I_{\text {charge }}$ with the operating conditions, $\mathrm{V}_{\text {in }}=30 \mathrm{~V}$ peak, $\mathrm{F}_{\text {in }}=1.85 \mathrm{~Hz}, \mathrm{R}_{\text {in }}=15 \Omega, \mathrm{I}_{\text {in }}=2$ A peak, $\mathrm{P}_{\text {inavg }}=13 \mathrm{~W}, \mathrm{P}_{\text {inmax }}$ $=60 \mathrm{~W}$. It is shown in the figure that the input current, $\mathrm{I}_{\mathrm{in}}$, is a half wave rectified sinusoid. The variable $R_{\text {in }}$ controlled Boost converter is emulating an input resistor of $15 \Omega$ with an average input power of approximately 13 watts and peak power around 60 watts. As the peak input power is higher than $\mathrm{P}_{\text {outmax }}$ the ESC will be used to temporarily store the extra energy, shown by the waveform $\mathrm{V}_{\text {boost }}$. During the input current cycle the MEECC is regulating the charging current to the battery, $\mathrm{I}_{\text {charge }}$ and it does not exceed the 2 Amp charging current specification. The input current profile continues to extract power and store the energy in the ESC regardless of how much power is being stored in the battery pack. Once the input power has decreased, the Buck converter continues to charge the battery at the 2 Amp CC rate with the extracted energy stored in the ESC. The battery stops charging once the voltage of the ESC, $\mathrm{V}_{\text {Boost }}$, has reached the minimum voltage level, $\mathrm{V}_{\text {Boostmin. }}$ During the CC charging mode the battery remains under the CV charging limit of 8.4 volts, shown by the battery voltage waveform $\mathrm{V}_{\text {batt. }}$ 


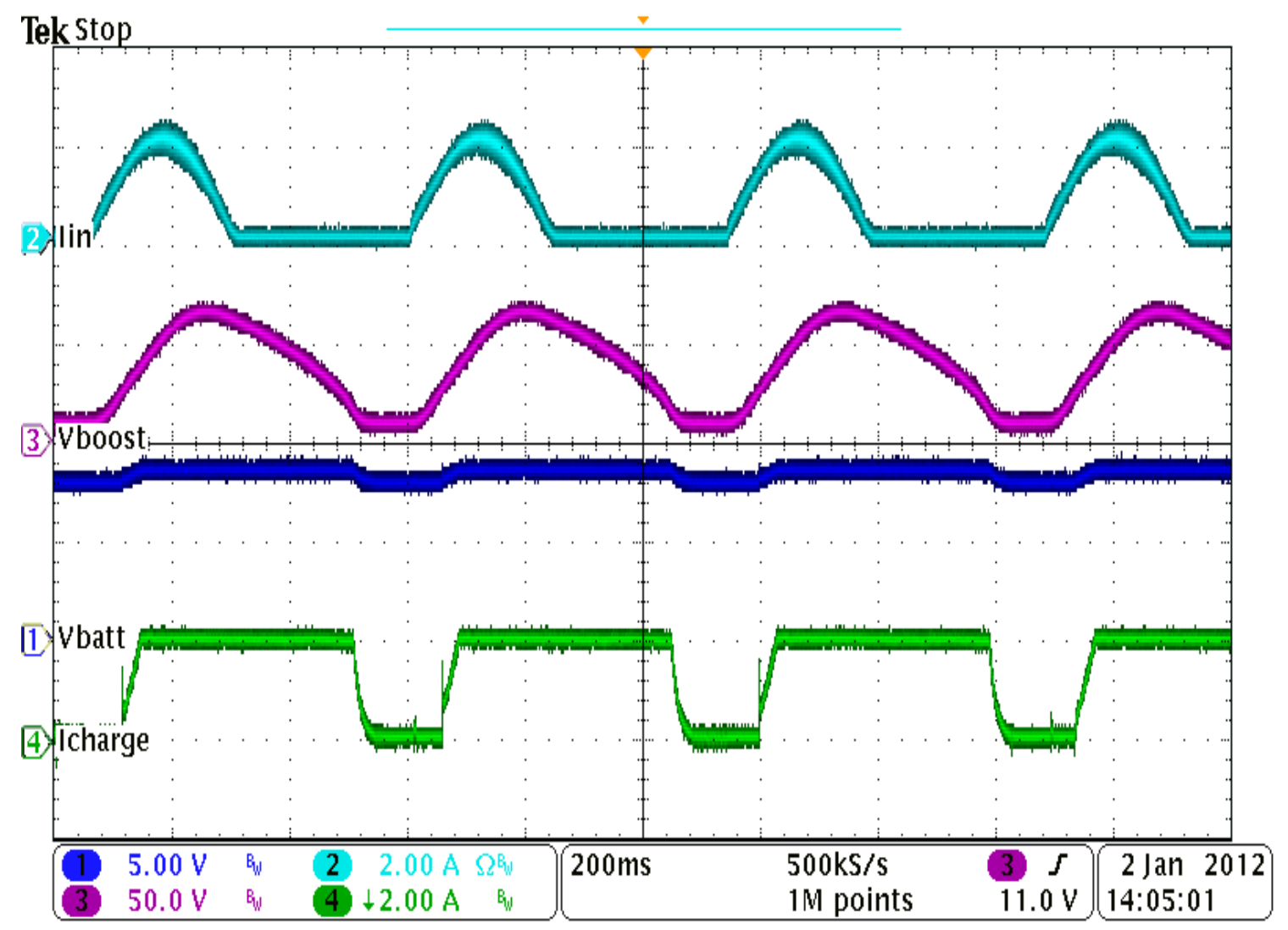

Figure 5-4 - Full system operation, $R_{\text {in }}=15 \Omega$, Buck in CC mode 
As the battery continues to charge in CC mode the voltage will increase until it reaches the 8.4 volt threshold, once the battery reaches this voltage it must keep the voltage constant and reduce the charging current to continue to charge the battery. Shown in Figure 5-5 is the same input operating conditions as Figure 5-4, however, the battery has reached the point where it is charging in CV mode. This can be observed by both the charging current and the charging voltage waveforms, $\mathrm{I}_{\text {charge }}$ and $\mathrm{V}_{\text {batt }}$. The charging current is less than the CC rate of $2 \mathrm{~A}$ and the charging voltage is being regulated at 8.4 volts. During this CV operation the Boost converter is still emulating a constant resistor value of $15 \Omega$ and extracting power and storing the excess energy temporarily in the ESC, represented by the voltage $\mathrm{V}_{\text {boost }}$. After the input power has decreased the battery continues to charge at the CV rate until the ESC's energy has been depleted.

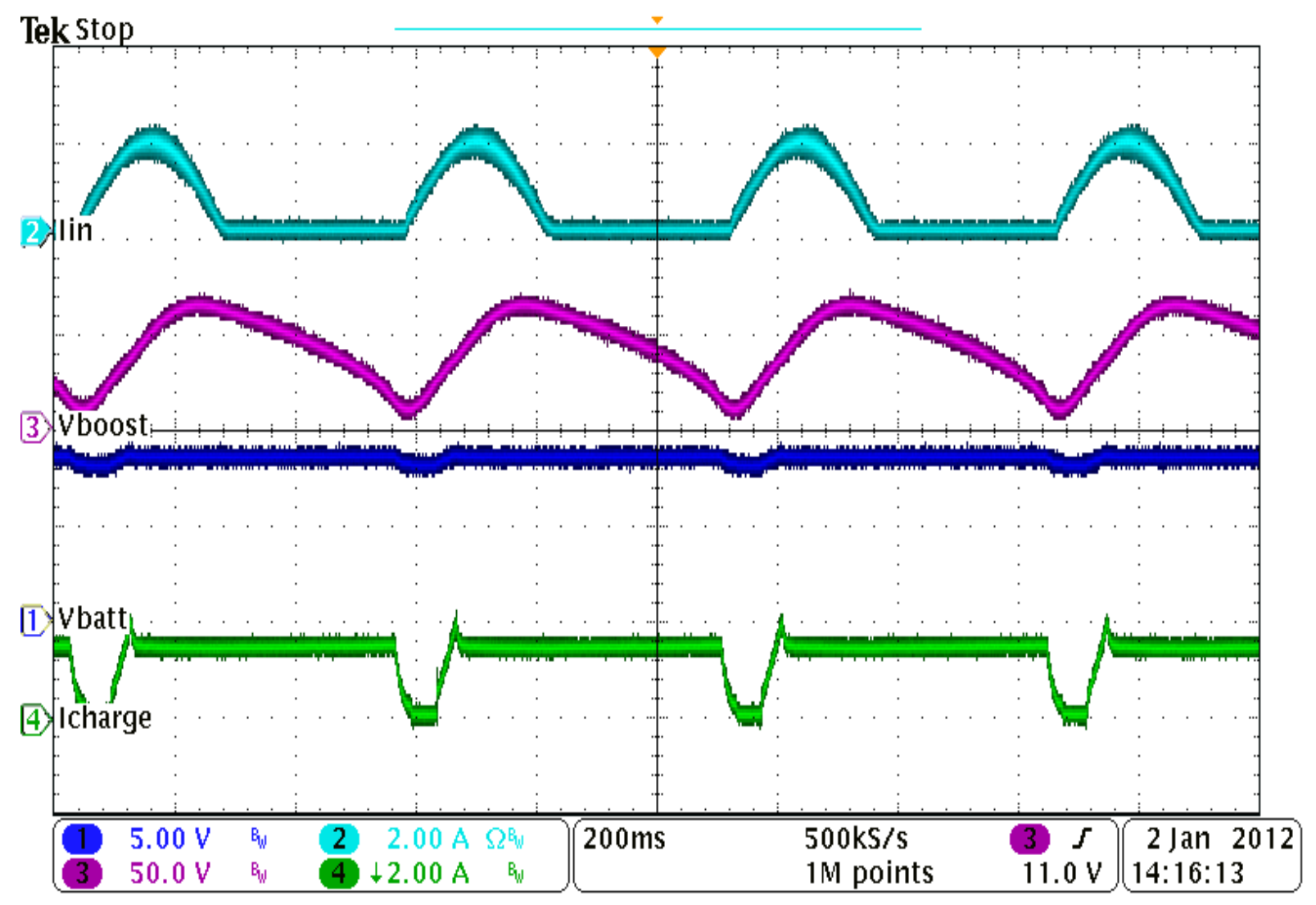

Figure 5-5 - Full System operation, $R_{\text {in }}=15 \Omega$, Buck in CV mode 
From both Figure 5-4 and Figure 5-5, it can be seen that the $\mathrm{R}_{\mathrm{in}}$ Controlled Boost converter continues to extract power from the input even after the batteries are charging at their maximum rate. As this extra energy is not lost but rather temporarily stored in the ESC to be later transferred to the batteries, the batteries will charge faster than with a single stage system that cannot store this extra energy. In order to determine how much more effective the proposed system is at extracting power compared to a single stage system without intermediate energy storage, a mechanical test rig that can mimic the power input profile that a human would generate from walking is needed. Such a mechanical test rig has been designed and described in the next section.

\subsubsection{Mechanical Test Rig}

In order to test the PEM under different operating conditions an input power source for the system is needed. The mechanical test rig will replicate the power profile generated by a human walking. The mechanical system will remove any variations that the human would introduce into the input power profile. This will allow us to compare the PEM under different test conditions while using the same input power profile. The mechanical test rig was designed to closely approximate the input that would be typical for a human walking. The test rig was also designed to 'feel' the effect of the generator being loaded by the PEM and reduce rpm, similar to how a human would feel the resistance of the generator being loaded and the rpm of the generator would decrease. The mechanical test rig consists of a three-phase generator coupled to a dc motor. The three phase generator shown on the right of Figure 5-6 is the same generator that is used in the portable human generator to provide the PEM with the same generator characteristics that the portable human energy harvester generator would. The DC motor, shown on the left of Figure 5-6 can be adjusted to how much the motor slows down while being loaded by adjusting the resistor value in series with the DC motor $\mathrm{R}_{\mathrm{ext}}$. The electrical schematic of the Mechanical Test Rig is shown in Figure 5-7. As $\mathrm{R}_{\mathrm{ext}}$ is increased the motor will slow down more with a given load. The mechanical test rig was 
calibrated to provide the same peak output voltage and average power level that a human would generate when wearing the portable human generating device under the same loading conditions for the generator, $\mathrm{R}_{\mathrm{ext}}=7.5 \Omega$.

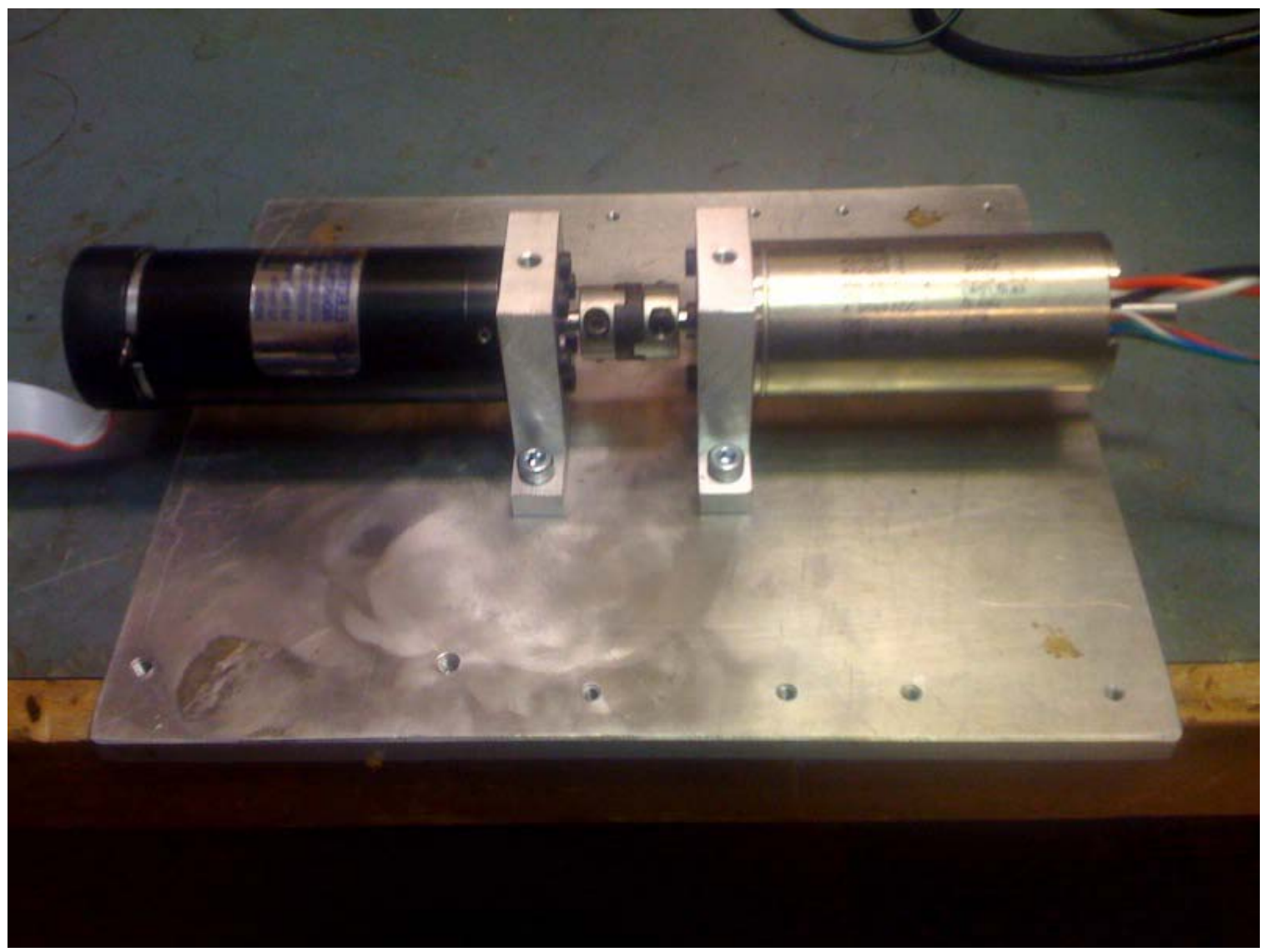

Figure 5-6 - Mechanical Test Rig with DC motor drive on the left and 3-phase generator shown on the right 


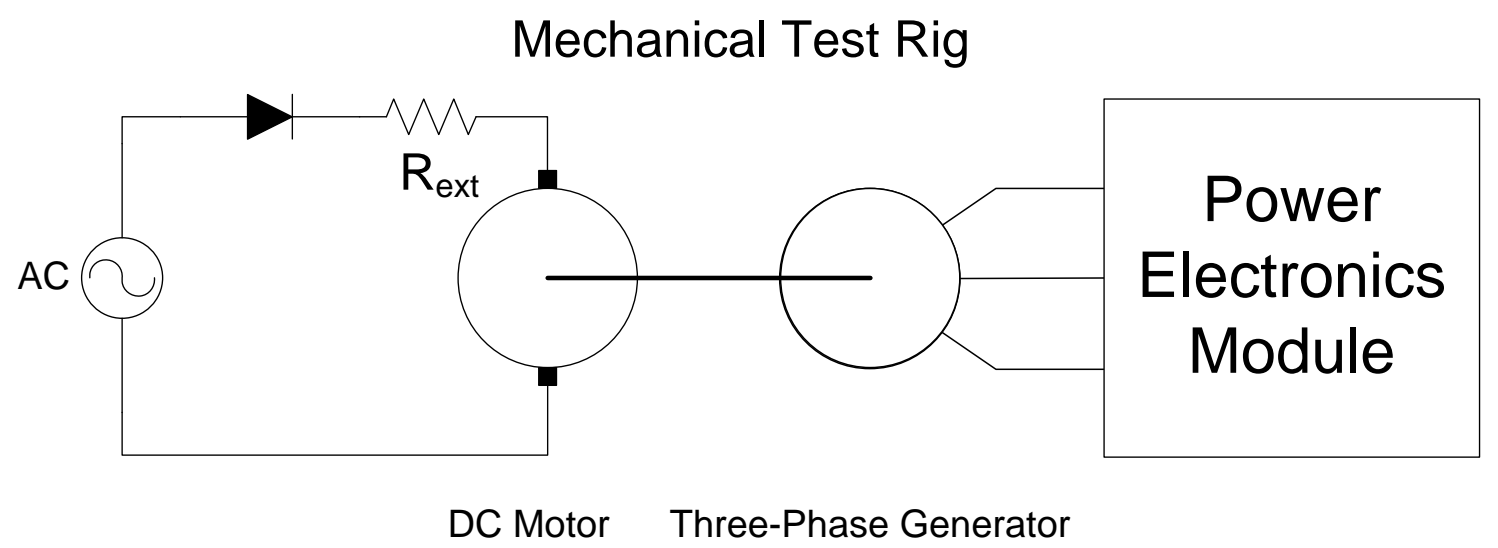

\section{Figure 5-7 - Electrical Schematic of Mechanical Test Rig}

The mechanical test rig will more accurately produce the input power waveforms similar to a human wearing the portable human generator than an AC input source would. The mechanical test rig will allow us to determine how much more power can be extracted with the proposed PEM then with a single stage system while the input power is a power limited source.

The mechanical test rig is used to generate the input power to test how much power is extracted while using the proposed PEM system. The test waveforms are shown in Figure 5-8 with $V_{\text {in }}=25$ $\mathrm{V}$, the Constant $\mathrm{R}_{\mathrm{in}}$ Controlled Boost converter is shown to be drawing current according to a constant emulated $\mathrm{R}_{\mathrm{in}}$ value for the entire input voltage waveform. When the input power is greater than the output power to the battery the excess energy is stored in the ESC, shown by $\mathrm{V}_{\text {boost. }}$ The average output power using the proposed PEM with the ESC is $9 \mathrm{~W}$ under these operating conditions. 


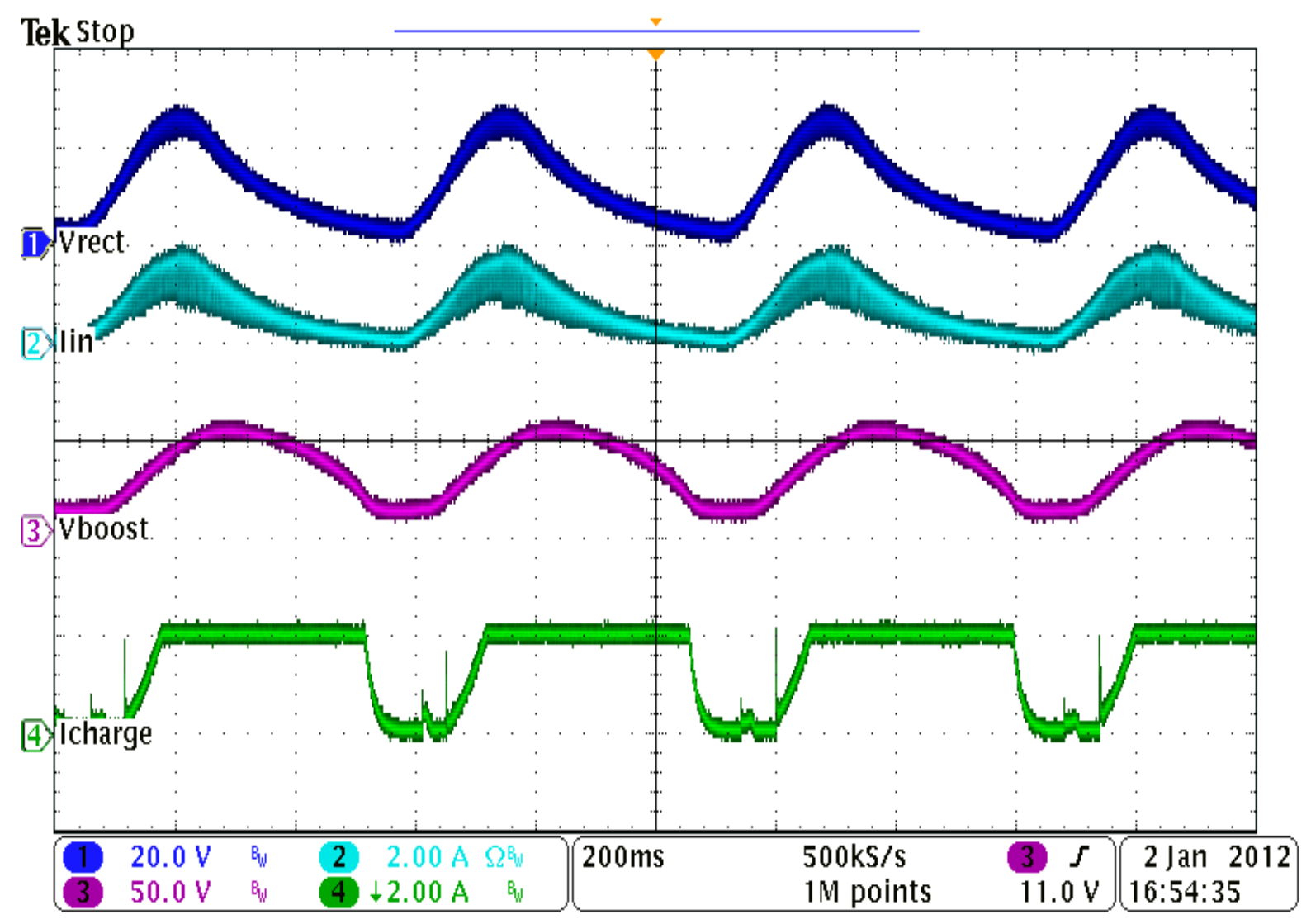

\section{Figure 5-8 - PEM excited by Mechanical Test Rig}

The $\mathrm{R}_{\text {in }}$ Controlled Boost converter was bypassed and the ESC was removed to test how much power a single stage Buck converter could extract from the same power limited source under the same excitation conditions, $\mathrm{V}_{\text {in }}=25 \mathrm{~V}$. The Buck charger was used to charge the battery directly from the rectified input voltage to get the waveforms shown in Figure 5-9. The Buck charger must still abide by the charging regulations of the battery pack and must not exceed the 2A charge rate.

As can be observed in the $\mathrm{I}_{\text {in }}$ waveform the input current is not following an emulated resistance as in the case with the $\mathrm{R}_{\mathrm{in}}$ Controlled Boost converter enabled, the input current must decrease when the input power is greater than the output power to the battery. The result is less power is extracted 
from the generator and therefore the battery pack is charged at a slower rate. The average output power to the battery is measured at $6.1 \mathrm{~W}$, only $66 \%$ of $9 \mathrm{~W}$.

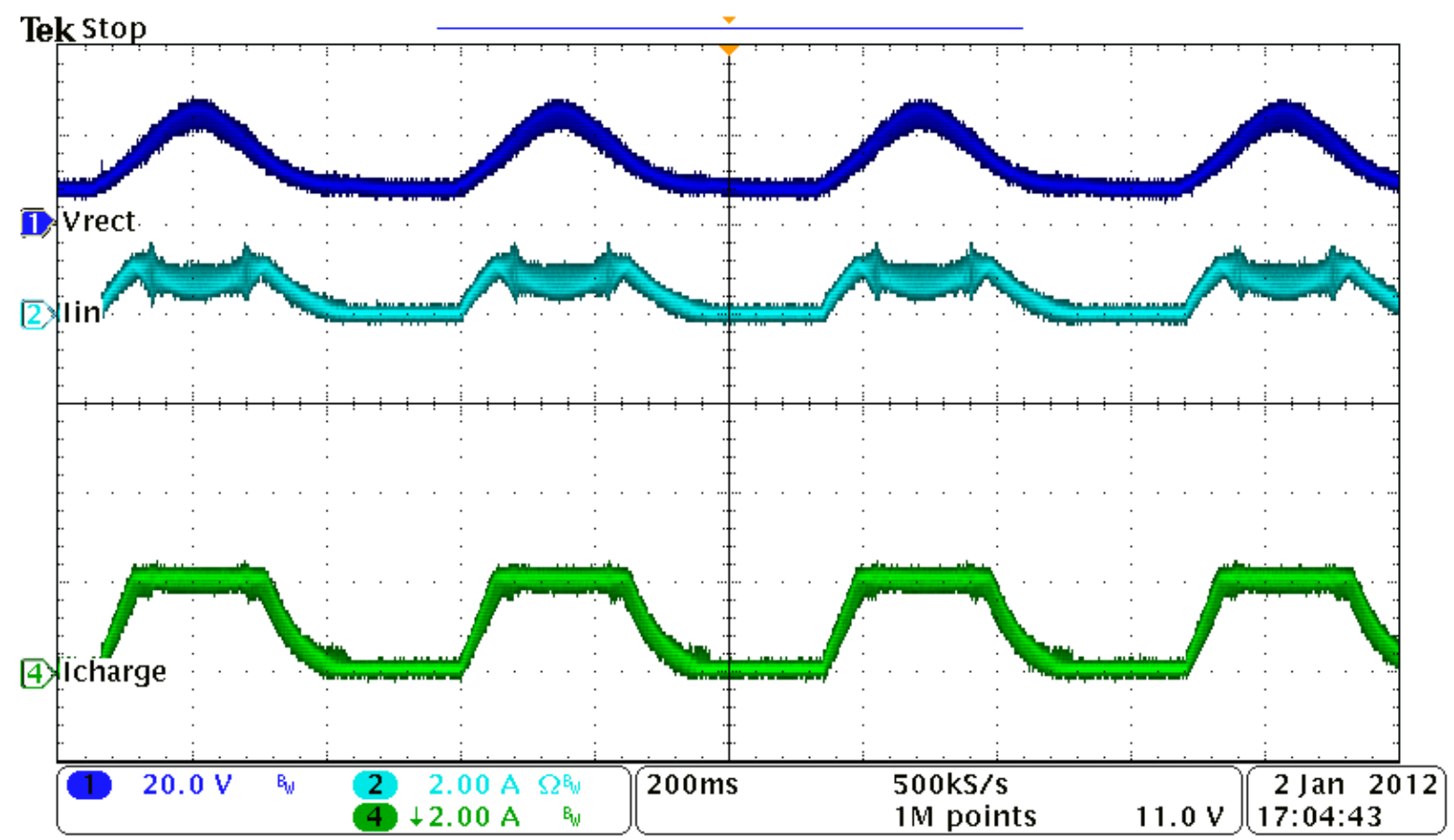

\section{Figure 5-9 - Single Stage Buck excited by Mechanical Test Rig}

With the $\mathrm{R}_{\text {in }}$ Controlled Boost converter, the ESC and the Buck Maximum Energy Extraction and Charge Controller the battery pack will charge 150\% faster than with a single stage Buck converter. The $\mathrm{R}_{\text {in }}$ Controlled Boost converter also provides the advantage of being able to draw current over the entire input voltage waveform increasing the energy extraction as well as allowing the user to control the input current.

The PEM DC electrical efficiency is measured similar to the Boost converter and Buck converter efficiencies, with the Boost operating in Constant $\mathrm{R}_{\text {in }}$ Control mode and the Buck charging in CC mode. The PEM DC efficiency is the overall efficiency of both the Boost converter and Buck converter combined measured from the input power of the rectifier to the output power of the 
battery pack. The input voltage is kept constant with $\mathrm{V}_{\text {in }}=20 \mathrm{~V} \mathrm{DC}$ and the power level is varied by varying the Boost $R_{\text {in }}$ value. The efficiency vs. input power is shown in Figure 5-10 for the Boost converter, the Buck converter and the complete PEM. The Buck MEECC is responsible for the regulation of $\mathrm{V}_{\text {boost }}$ as well as the output power level to the battery. The peak efficiency was measured at $87.9 \%$ with an emulated input resistance of $40 \Omega$ and an input power of 10 watts.

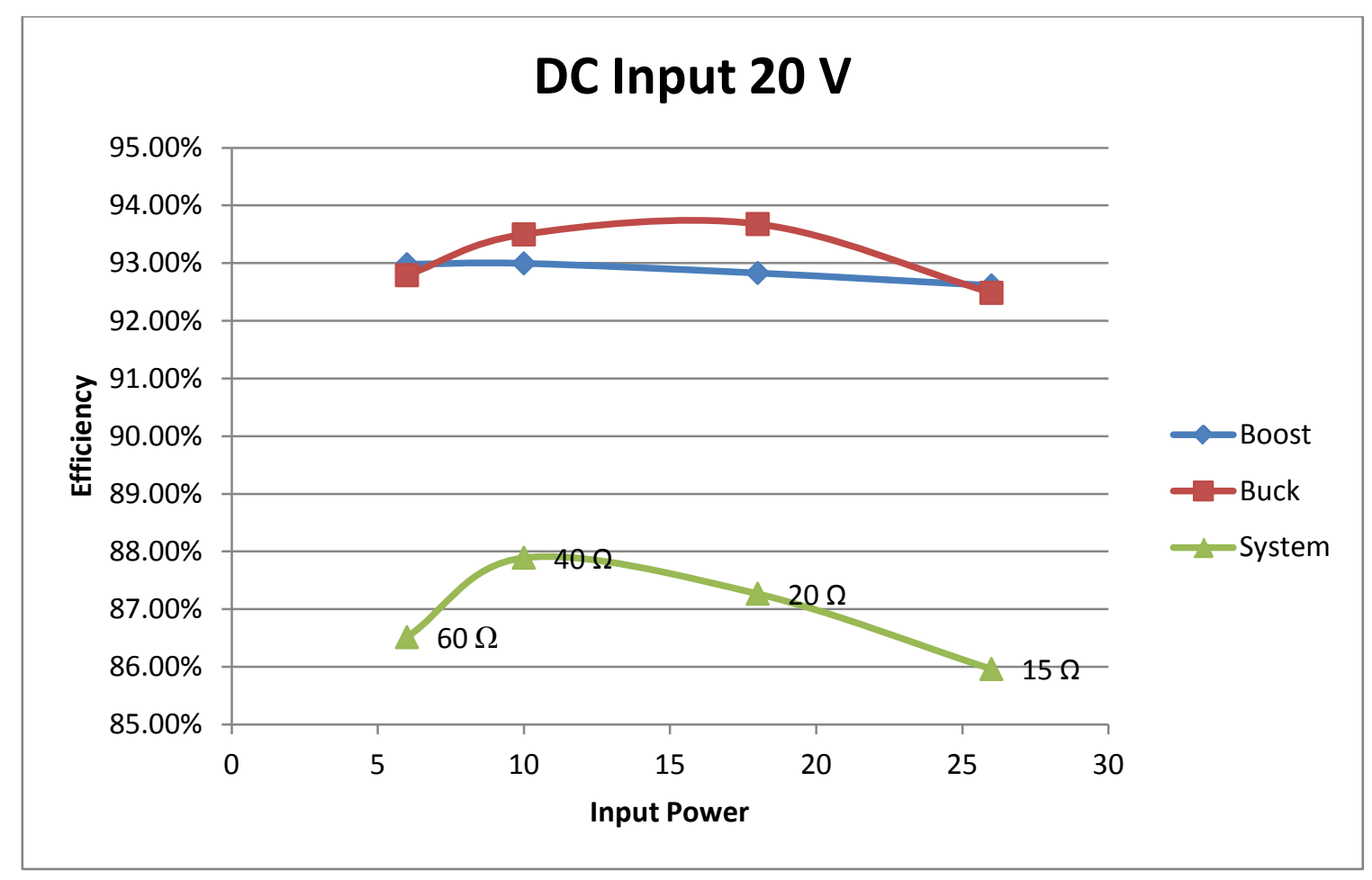

\section{Figure 5-10 - Complete System DC Efficiency}

The DC efficiency is measured with a constant DC input voltage of $20 \mathrm{~V}$, this is, however, only a single point along the AC input voltage waveform that the PEM would be operating.

A much more significant measure of efficiency is the AC efficiency of the converter when the input waveform is varying similar to what would be expected from a human generating the electricity. The AC efficiency of the PEM is measured with different input voltage levels, $V_{\text {inpeak }}=15 \mathrm{~V}, 20 \mathrm{~V}$, $30 \mathrm{~V}, \mathrm{~F}_{\mathrm{in}}=1.85 \mathrm{~Hz}$. The $\mathrm{R}_{\mathrm{in}}$ value is varied to change the power level of the converter under the 
different input voltage conditions. The efficiency is plotted versus emulated input resistor, $\mathrm{R}_{\mathrm{in}}$ as shown in Figure 5-11 and the input power level of each measurement is given.

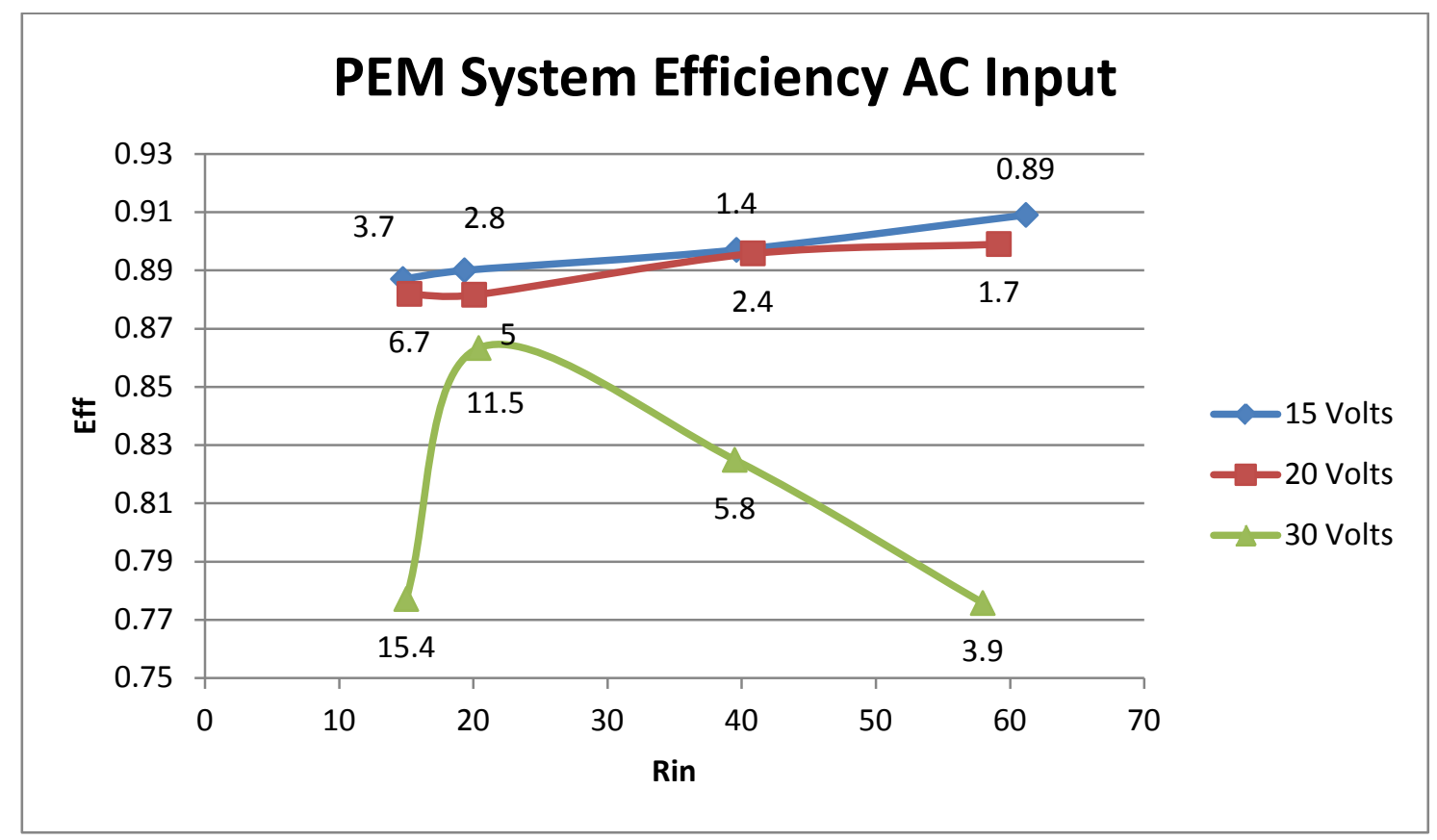

Figure 5-11 - PEM System Efficiency with AC Input

The peak efficiency for $V_{\text {in }}=15 \mathrm{~V}$ is $91 \%$ at $R_{\text {in }}=60 \Omega, P_{\text {in }}=0.89 \mathrm{~W}$, the peak efficiency for $V_{\text {in }}=$ $20 \mathrm{~V}$ is $90 \%$ at $R_{\text {in }}=60 \Omega, P_{\text {in }}=1.7 \mathrm{~W}$, the peak efficiency for $V_{\text {in }}=30 \mathrm{~V}$ is $86 \%$ at $R_{\text {in }}=20 \Omega, P_{\text {in }}$ $=11.5 \mathrm{~W}$.

\subsection{Conclusion}

This chapter presented the complete system design considerations. This included the design and optimization of the embedded system software that was running on the DSP. The software ISR and ADC sampling timing chart was provided along with a software flow diagram to calculate the delay associated with each of the sampled signals with the most care being taken with the placement of the time critical tasks and controllers. Optimization of the code, as well as turning off the DSP peripherals that weren’t being used reduced the power consumption of the DSP by $21 \%$. A design chart was provided to aid in the selection of the capacity and voltage rating of the ESC required to 
store all of the excess energy during the CC charging mode of the battery from $60-120 \%$ nominal input power. Experimental tests were conducted and waveforms were captured to show that both the $\mathrm{R}_{\mathrm{in}}$ Controlled Boost converter and the Buck Maximum Energy Extraction and Charge Controller can work together to meet all of the design requirements that were required by the PEM. A mechanical test rig was designed and calibrated to mimic the input power profile that a human wearing the human energy harvester would generate, including the 'feeling' of the load, without the variance a human test subject would introduce into the power input profile. This mechanical test rig was used as the input generator to determine how effective the PEM system is at extracting power from the generator compared to a similar single staged design without a temporary energy storage solution. The proposed PEM was able to extract 50\% more power than the single stage system resulting in the battery pack charging 50\% faster. The PEM DC efficiency was measured with the Boost converter emulating different $\mathrm{R}_{\mathrm{in}}$ values with an input voltage of $20 \mathrm{~V}$ DC. The efficiency of the Boost converter and the Buck converter were compared to the total system efficiency with the total system peak efficiency being $87.9 \%$ efficient at an emulated load of $40 \Omega$. The AC efficiency was also measured for a more realistic input voltage variation, the peak efficiency was measured $91 \%$ with $V_{\text {in }}=15 \mathrm{~V}$ at $R_{\text {in }}=60 \Omega$. 


\section{Chapter 6}

\section{Conclusion and Future Work}

\subsection{Conclusions}

The objective of this thesis was to design a power converter and control algorithms to extract the maximum possible amount of energy from a human powered energy harvester while not making the load felt by the user too cumbersome. The extracted power must be safely stored in an appropriately sized battery for later use. This objective was broken into two design goals and a twostage converter, one converter and controller per design goal. The first design goal to reach the thesis objective was that the first stage Boost converter must extract power from the generator while controlling the input power as set by the user. This objective was accomplished by controlling the Boost converter with three modes of control, all of which are variations on controlling the emulated input resistor value of the Boost converter based on the user input value. With this control the Boost converter will draw power from the input according to the user's desired input level regardless of the output voltage of the Boost converter. This objective was met and verified by experimental waveforms and results.

The second design goal to reach the thesis objective was to control the Buck converter to maximize the power transfer from the output of the first stage and Energy Storage Capacitor into the battery pack while staying inside the charging limitations of an appropriately sized battery pack. This objective was accomplished by the design of the Maximum Energy Extraction and Charge Control algorithm, in which the voltage of the energy storage capacitor was minimized by transferring all available energy into the battery pack while not exceeding the charging current or charging voltage of the battery pack. This objective was met and verified by experimental waveforms and results. 


\subsection{Summary}

Chapter 1 introduced the subject of human energy harvesting and the many different variations of energy harvesting devices and power ratings of the generators. The power electronics that are used to store the harvested energy was analyzed and the shortcomings of the designs were identified. This chapter establishes motivation and set out the objectives for the research contributions presented in this thesis.

Chapter 2 presented the power electronic design was proposed to overcome the shortcomings of the previous designs while meeting the design requirements of a new version of generator designed by collaborators in the mechanical engineering department. The design identified what the controllers must accomplish and how the energy storage capacitor can be used to reduce the size of battery needed while not sacrificing potential energy harvest.

Chapter 3 introduced the control strategy used to control the Boost converter to achieve the first of the design objectives of the thesis: to extract power from the generator according to the user's desired input level regardless of the output voltage of the Boost converter maximizing energy harvest from the generator. The control was comprised of three different operating modes all of which are variations on controlling the emulated input resistor value based on the user's desired input power level. A digitally controlled prototype was designed and experimental results were presented to test the controller's functionality and validate that the design can meet the thesis design goals. The transfer function of the Boost average current mode was discretized and included the effects of the digitization. A Voltage Adaptive Gain compensator was designed to help achieve better dynamic performance over the wide output voltage range of the Boost converter. The DC 
efficiency of the Boost converter was presented with the highest efficiency being 93\% with an emulated resistance of $40 \Omega$ and an input power of $10 \mathrm{~W}$.

Chapter 4 introduced the Maximum Energy Extraction and Charge Controller that was used to meet the second design goal of the thesis: to control the Buck converter to maximize the power transfer from the output of the first stage and Energy Storage Capacitor into the battery pack while staying inside the charging limitations of an appropriately sized battery pack. The MEECC controller consisted of four sub controllers, two of which controlled the battery charging current and voltage, the other two regulating the voltage of the ESC to the minimum voltage. The transfer function of the voltage loop and current loop was derived. A Voltage Adaptive Gain controller was used to optimize the dynamic response of the current loop over the wide input voltage range of the Buck converter. A digitally controlled prototype was designed a built with experimental results to show the effectiveness of the MEECC controller in meeting the second design goal of the thesis. A method of driving the synchronous MOSFET in the Buck converter was used to eliminate the need of an isolated voltage supply for the Control MOSFET driver. The efficiency of the Buck converter was measured with the peak efficiency $93.7 \%$ at a power level of 18 watts.

Chapter 5 detailed the design process for the sections of the power electronics module that were not covered in Chapters 3 and 4. This included the considerations of timing and power consumption of the embedded system software as well at time delay associated with the sample and hold process for sampled signals. A software flow diagram was provided as well as a timing diagram. A design guide for selection of the ESC capacitance and voltage rating was created for power levels of $60 \%$ $120 \%$ of the nominal power range expected. Experimental results were captured to verify that the two converters each achieving separate design goals operated together to meet the overall design 
objective of the PEM: 1) to maximize the power harvested from the human energy harvester according to the user’s desired input power profile and 2) store the extracted energy into appropriately sized batteries while not exceeding the charging limitations of the battery. The design and controllers of the PEM were found to meet this thesis objective. Test results were conducted to verify that a two stage design with energy storage capability would extract more power from the human energy harvester then a single stage design without temporary energy storage capability. It was found that $50 \%$ more power can be extracted with the use of the ESC and controllers. The total PEM DC efficiency was measured of both stages with a peak efficiency of $87.9 \%$ efficiency at an emulated load of $40 \Omega$. An AC efficiency was also measured for the PEM and the peak efficiency was measured at $91 \%$ with $\mathrm{V}_{\text {in }}=15 \mathrm{~V}$ at $\mathrm{R}_{\text {in }}=60 \Omega$.

\subsection{Future Work}

The following suggestions for future work are made:

- The P-Controller used in the Boost voltage controller could be redesigned as a modified PIController. The controller would have different integral saturation limits for positive and negative error, removing the possibility of undershooting the minimum Boost voltage and removing the steady state error while regulating the voltage.

- Different topologies can be explored to replace the Boost converter with a bridgeless topology, removing the three-phase diode rectifier improving the efficiency of the first stage.

- The requirement that the minimum Boost voltage is $110 \%$ of the greater of the input voltage or battery voltage could be further explored in order to find the minimum limitation of the value. A reduction of the minimum Boost voltage would improve the efficiency of both stages. 
- A new three-phase topology that can draw three-phase sinusoidal current from the generator could be designed to reduce the conduction losses created by the internal winding resistance of the generator.

- The embedded software could be further optimized to reduce the clock frequency of the DSP, saving power consumption increasing energy harvest.

- A maximum power point tracking algorithm could sweep the emulated resistor values to maximize the charging power of the battery. 


\section{References}

[1] T. Starner and J. A. Paradiso, "Human Generated Power for Mobile Electronics," in Low Power Electronics Design, C. Piguet, Ed., ed. CRC Press: Boca Raton, 2004.

[2] K. Matsuzawa and M. Saka., "Seiko human powered quartz watch," in Prospector IX: Human-Powered Systems Technologies, M. Rose, Ed., ed. Auburn, AL: Space Power Institute, Auburn University, 1997, pp. 359-384.

[3] J. A. Paradiso, "Systems for human-powered mobile computing," in Design Automation Conference, 2006 43rd ACM/IEEE, 2006, pp. 645-650.

[4] W. Guanghui, L. Cheng, H. Hofmann, and L. Rome, "Power electronic circuitry for energy harvesting backpack," in Energy Conversion Congress and Exposition, 2009. ECCE 2009. IEEE, 2009, pp. 3544-3549.

[5] J. F. Antaki, Gina E. Bertocci, E. C. Green, A. Nadeem, T. Rintoul, R. L. Kormos, and B. P. Griffith, "A Gait-Powered Autologous Battery Charging System for Artificial Organs," American Society of Artificial Internal Organs, vol. 41(3), pp. M588-M595, July Setember 1995.

[6] S. Ben Ayed, F. Najar, and A. Abdelkefi, "Shape improvement for piezoelectric energy harvesting applications," in Signals, Circuits and Systems (SCS), 2009 3rd International Conference on, 2009, pp. 1-6.

[7] M. Duffy and D. Carroll, "Electromagnetic generators for power harvesting," in Power Electronics Specialists Conference, 2004. PESC 04. 2004 IEEE 35th Annual, 2004, pp. 2075-2081 Vol.3.

[8] J. A. Paradiso and T. Starner, "Energy scavenging for mobile and wireless electronics," Pervasive Computing, IEEE, vol. 4, pp. 18-27, 2005.

[9] Z. Peng, C. Hao, Y. Zhi, and A. Khaligh, "Unconventional wearable energy harvesting from human horizontal foot motion," in Applied Power Electronics Conference and Exposition (APEC), 2011 Twenty-Sixth Annual IEEE, 2011, pp. 258-264.

[10] J. M. Donelan, Q. Li, V. Naing, J. A. Hoffer, D. J. Weber, and A. D. Kuo, "Biomechanical Energy Harvesting: Generating Electricity During Walking with Minimal User Effort," Science, vol. 319, pp. 807-10, Feb 82008.

[11] Q. Li, V. Naing, J. A. Hoffer, D. J. Weber, A. D. Kuo, and J. M. Donelan, "Biomechanical energy harvesting: Apparatus and method," in Robotics and Automation, 2008. ICRA 2008. IEEE International Conference on, 2008, pp. 3672-3677. 
[12] J. Granstrom and J. Feenstra, "Energy harvesting from a backpack instrumented with piezoelectric shoulder straps," Smart Materials \& Structures, vol. 16, pp. 1810-1820, Oct 2007.

[13] L. C. Rome, L. Flynn, E. M. Goldman, and T. D. Yoo, "Generating electricity while walking with loads," Science, vol. 309, pp. 1725-1728, Sep 92005.

[14] J. Elmes, V. Gaydarzhiev, A. Mensah, K. Rustom, J. Shen, and I. Batarseh, "Maximum Energy Harvesting Control for Oscillating Energy Harvesting Systems," in Power Electronics Specialists Conference, 2007. PESC 2007. IEEE, 2007, pp. 2792-2798.

[15] D. Kuo, "Biophysics: Harvesting Energy by Improving the Economy of Human Walking," Science, vol. 309, pp. 1686-7, Sep 92005.

[16] C. Xinping, C. Wen-Jen, K. Ya-Chin, and L. Yi-Kuen, "Electromagnetic Energy Harvesting Circuit With Feedforward and Feedback DC\&ndash;DC PWM Boost Converter for Vibration Power Generator System," Power Electronics, IEEE Transactions on, vol. 22, pp. 679-685, 2007.

[17] I. Buchmann. Battery University [Online]. Available: http://batteryuniversity.com/

[18] Bren-Tronics, Inc. Datasheet of BB-2590/U [Online]. Available: http://www.brentronics.com/resource/datasheets/DS\%20BT-70791A\%20Rev\%20E.pdf

[19] D. Linden and T. B. Reddy, Handbook of Batteries (3rd Edition): McGraw-Hill, 2002.

[20] Woodbank Communications Ltd. "Relative Energy Density of Some Common Secondary Cell Chemistries" [Online]. Available: http://www.mpoweruk.com/chemistries.htm

[21] S. Dearborn, "Lithium-Ion Battery Charging: Techniques and Trade-offs," ed: Microchip Technologies. Available:

http://techtrain.microchip.com/webseminars/ArchivedDetail.aspx?Active=44.

[22] Union Battery. Datasheet of 585460 Li-Polymer Battery Pack [Online]. Available: http://www.sparkfun.com/datasheets/Batteries/UnionBattery-2000mAh.pdf

[23] C. P. Basso, Switch-Mode Power Supplies: McGraw-Hill, 2008.

[24] B. Hauke, "Basic Calculation of a Boost Converter's Power Stage," Texas Instruments, Application Report November 2009.

[25] "Altium Designer 10," Altium, http://products.live.altium.com/.

[26] L. A. C. Lopes, "Small Signal Model of Boost DC-DC Converter Operating in CCM," Concordia University, Design Example Available: http://users.encs.concordia.ca/ lalopes/Courses/ELEC6481-W08/Example234.pdf2008. 
[27] S. Choudhury, "DSP Implementation of an average current mode controlled Power Factor Corrention Converter," presented at the International Power Electronics Technolofy Confrence, 2003.

[28] S. Choudhury, "Designing a TMS320F280x Based Digitally Controlled dc-dc switching power supply," Texas Instruments, Application Report July 2005.

[29] J. D. P. Gene F. Franklin, Michael L. Workman, Digital Control Of Dynamic Systems, 1990.

[30] D. R. B. Ridley, Power Supply Design vol. 1: Control: Ridley Designs, 2011.

[31] K. Ogata, "Modern Control Engineering," 4th Edition ed: Prentice Hall, 2001.

[32] Linear Technology, Datasheet of LTC4444-5 [Online]. Available: http://cds.linear.com/docs/Datasheet/44445fc.pdf

[33] A. I. Pressman, K. Billings, and T. Morey, Switching Power Supply Design, 3rd ed.: McGraw-Hill, 2009.

[34] S. Heath. (2003, January 20, 2012). Embedded systems design (2nd ed.) [Online]. Available: http://books.google.com/books?id=BjNZXwH7HlkC\&pg=PA2

[35] Texas Instruments. Data Manual for TMS320F2808 Digital Signal Processors [Online]. Available: http://www.ti.com/lit/gpn/tms320f2808

[36] Texas Instruments. C280xx Digital Power Software Solutions - DC-DC Buck converter using High Resolution ePWM [Online]. Available: http://www.ti.com/lit/zip/sprc229

[37] Texas Instruments. TMS320x280x, 2801x, 2804x DSP Analog-to-Digital Converter (ADC) [Online]. Available: http://www.ti.com/lit/ug/spru716d/spru716d.pdf

[38] Texas Instruments. TMS320C28x CPU and Instruction Set Reference Guide [Online]. Available: http://www.ti.com/lit/ug/spru430e/spru430e.pdf

[39] Texas Instruments. TMS320x280x, 2801x, 2804x DSP System Control and Interrupts [Online]. Available: http://www.ti.com/lit/ug/spru712g/spru712g.pdf

[40] Texas Instruments. TMS320x280x, 2801x, 2804x High Resolution Pulse Width Modulator [Online]. Available: http://www.ti.com/lit/ug/spru924f/spru924f.pdf

[41] Texas Instruments. TMS320x280x, 2801x, 2804x Enhanced Pulse Width Modulator (ePWM) Module [Online]. Available: http://www.ti.com/lit/ug/spru791f/spru791f.pdf 